Astronomy Unit

School of Physics and Astronomy

Queen Mary University of London

\title{
Pickup Ion Processes Associated with Spacecraft Thrusters Implications for Solar Probe Plus
}

Adam Clemens

Submitted in partial fulfillment of the requirements of the Degree of Doctor of Philosophy 


\section{Declaration}

I, Adam Clemens, confirm that the research included within this thesis is my own work or that where it has been carried out in collaboration with, or supported by others, that this is duly acknowledged below and my contribution indicated. Previously published material is also acknowledged below.

I attest that I have exercised reasonable care to ensure that the work is original, and does not to the best of my knowledge break any UK law, infringe any third party's copyright or other Intellectual Property Right, or contain any confidential material.

I accept that the College has the right to use plagiarism detection software to check the electronic version of the thesis.

I confirm that this thesis has not been previously submitted for the award of a degree by this or any other university.

The copyright of this thesis rests with the author and no quotation from it or information derived from it may be published without the prior written consent of the author.

Signature:

Date: $24 / 09 / 2015$ 


\begin{abstract}
Chemical thrusters are widely used in spacecraft for attitude control and orbital manoeuvres. They produce a plume of neutral gas which produces ions via photoionisation and charge exchange. Measurements of local plasma properties will be affected by perturbations caused by the coupling between the newborn ions and the plasma. A model of neutral expansion has been used in conjunction with a fully three-dimensional hybrid code to study the evolution and ionisation over time of the neutral cloud produced by the firing of a mono-propellant hydrazine thruster as well as the interactions of the resulting ion cloud with the ambient solar wind. A parameter survey was performed for varying angles of injection and injection rates, particle kinetics were also investigated. Results are presented which show that the plasma in the region near to the spacecraft will be perturbed for an extended period of time with the formation of an interaction region around the spacecraft, a moderate amplitude density bow wave bounding the interaction region and evidence of an instability at the forefront of the interaction region which causes clumps of ions to be ejected from the main ion cloud quasi periodically and the ways in which these features are modified by the degree of solar wind mass loading and the relative orientation of the magnetic field to the angle of injection. This may affect Solar Probe Plus for a significant duration as data taking and delicate sensory equipment may be required to cease operation until local fluctuations return to a more moderate level. The scale of the fluctuations seen are dependent upon the duration of the thruster firing and the specific geometry and therefore effects may vary in-situ.
\end{abstract}




\section{Acknowledgements}

First and foremost I would like to thank Prof. David Burgess for all of the help and support he has given me over the last four years, without his friendly advice and guidance this thesis would never have been completed. Even when things took a turn for the worse his relentless optimism made me believe that perhaps it wasn't so bad and as it turned out we had the technology and we did rebuild!

I would also like to thank Chris Haynes for the many discussions, hints, tips and examples on everything MATLAB related without which I would likely still be smashing my head against today.

Of course I have to thank all of my fellow PhD students, both current and past for the company, discussions, help and most importantly for the beer and vodka at the SCR. You all helped keep me (kind of) sane!

I thank my mother and family for the continuous moral support and belief. Even when I lost faith in myself you were there to say "You can do it!"

Last, but certainly not least, I would like to thank Harriet. You helped, supported and encouraged me throughout this $\mathrm{PhD}$ and more in ways I probably never even knew, especially when the proverbial hit the fan. Thank you, I wouldn't be here without you.

This work was supported by the Science and Technology Facilities Council (STFC), grant number ST/J500860/1 


\section{Contents}

$\begin{array}{ll}\text { Abstract } & 3\end{array}$

$\begin{array}{ll}\text { Acknowledgements } & 4\end{array}$

$\begin{array}{lr}\text { 1. Introduction } & 8\end{array}$

2. The Physics of Pickup Ions 14

2.1. Introduction . . . . . . . . . . . . . . . . . . . . . . 14

2.2. Interstellar Pickup Ions . . . . . . . . . . . . . . . . . . . 21

2.2.1. Source of Neutral Particles . . . . . . . . . . . . . . . 21

2.2.2. Filtration and Other Effects . . . . . . . . . . . . . 23

2.2.3. Anomalous Cosmic Rays . . . . . . . . . . . . . . . . . 25

2.2.4. Shock Acceleration of Interstellar Pickup Ions . . . . . . . . . 27

2.3. Pickup Ions at Comets . . . . . . . . . . . . . . . . . . . . 30

2.4. Pickup Ions at Planets and Moons . . . . . . . . . . . . . . 39

2.4.1. Jupiter . . . . . . . . . . . . . . . . . . . . . . . . . . . . . . . . . . . . . . . . . . . .

2.4.2. Mars . . . . . . . . . . . . . . . . 41

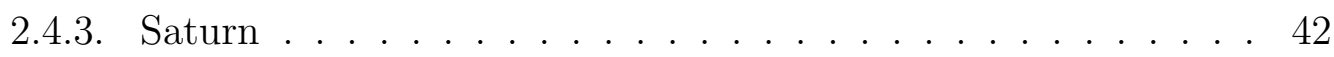

2.4.4. Mercury . . . . . . . . . . . . . . . 42

2.4.5. Earth . . . . . . . . . . . . . . . . . 43

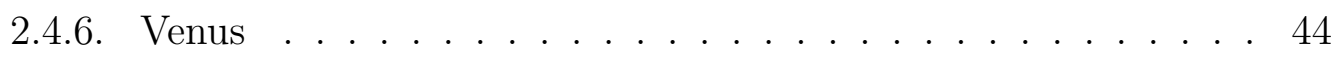

2.5. Active Release Experiments . . . . . . . . . . . . . . . . . . . . 45

2.5.1. CRRES . . . . . . . . . . . . . . . 46

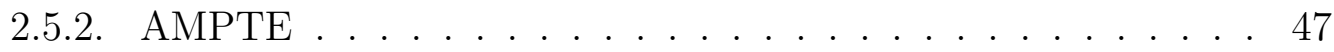

2.5.3. CRIT II . . . . . . . . . . . . . . . . . . . . . . . . . . . . . 49

3. Modelling Spacecraft Thruster Pickup Ions 52

3.1. Introduction . . . . . . . . . . . . . . . . . . 52

3.2. Neutral Expansion . . . . . . . . . . . . . . . . . . . . . . . . . . . 61

3.3. Ionisation . . . . . . . . . . . . . . . . . . . 72

3.4. Implementation . . . . . . . . . . . . . . . . . . . 74

3.5. Hybrid Model . . . . . . . . . . . . . . . . . . . . 76

4. Interaction of Spacecraft Thrusters with the Solar Wind - An Overview 80

4.1. Introduction . . . . . . . . . . . . . . . . . . 80

4.2. Simulation Setup . . . . . . . . . . . . . . . . . . . . . . . . . . . . 81

4.3. Results . . . . . . . . . . . . . . . . . . . . . . 82

4.4. Conclusions . . . . . . . . . . . . . . . . . . 100 
5. Interaction of Spacecraft Thrusters with the Solar Wind - A Pa$\begin{array}{lr}\text { rameter Survey } & 102\end{array}$

5.1. A Qualitative Overview . . . . . . . . . . . . . . . . . . . . 102

5.1.1. Injection at $45^{\circ}$ to the Initial Magnetic Field . . . . . . . . . 102

5.1.2. Injection at $0^{\circ}$ to the Initial Magnetic Field . . . . . . . . . . 104

5.1.3. Low Injection Rate at $90^{\circ}$ to the Initial Magnetic Field . . . . 105

5.1.4. High Injection Rate at $90^{\circ}$ to the Initial Magnetic Field . . . . 107

5.2. Magnetic Pileup . . . . . . . . . . . . . . . . . . . 108

5.2.1. Injection at $45^{\circ}$ to the Initial Magnetic Field . . . . . . . . . 108

5.2.2. Injection at $0^{\circ}$ to the Initial Magnetic Field . . . . . . . . . . 110

5.2.3. Low Injection Rate at $90^{\circ}$ to the Initial Magnetic Field . . . . 112

5.2.4. High Injection Rate at $90^{\circ}$ to the Initial Magnetic Field . . . . 114

5.3. Magnetic Field Draping . . . . . . . . . . . . . . . . . 114

5.3.1. Injection at $45^{\circ}$ to the Initial Magnetic Field . . . . . . . . . 114

5.3.2. Injection at $0^{\circ}$ to the Initial Magnetic Field . . . . . . . . . . 118

5.3.3. Low Injection Rate at $90^{\circ}$ to the Initial Magnetic Field . . . . 119

5.3.4. High Injection Rate at $90^{\circ}$ to the Initial Magnetic Field . . . . 119

5.4. Bow Waves . . . . . . . . . . . . . . . . . . . . . . . 122

5.4.1. Injection at $45^{\circ}$ to the Initial Magnetic Field . . . . . . . . 122

5.4.2. Injection at $0^{\circ}$ to the Initial Magnetic Field . . . . . . . . . . 125

5.4.3. Low Injection Rate at $90^{\circ}$ to the Initial Magnetic Field . . . . 126

5.4.4. High Injection Rate at $90^{\circ}$ to the Initial Magnetic Field . . . . 126

5.5. Interaction Region . . . . . . . . . . . . . . . . . . . . . 129

5.5.1. Injection at $45^{\circ}$ to the Initial Magnetic Field . . . . . . . . . 129

5.5.2. Injection at $0^{\circ}$ to the Initial Magnetic Field . . . . . . . . . 131

5.5.3. Low Injection Rate at $90^{\circ}$ to the Initial Magnetic Field . . . . 134

5.5.4. High Injection Rate at $90^{\circ}$ to the Initial Magnetic Field . . . . 134

5.6. Mass Loading . . . . . . . . . . . . . . . . . . . . . 137

5.6.1. Injection at $45^{\circ}$ to the Initial Magnetic Field . . . . . . . . 137

5.6.2. Injection at $0^{\circ}$ to the Initial Magnetic Field . . . . . . . . . 137

5.6.3. Low Injection Rate at $90^{\circ}$ to the Initial Magnetic Field . . . . 140

5.6.4. High Injection Rate at $90^{\circ}$ to the Initial Magnetic Field . . . . 140

6. Interaction of Spacecraft Thrusters with the Solar Wind - Particle Trajectories

6.1. Reference Simulation . . . . . . . . . . . . . . . . . . . . 143

6.2. Low Injection Rate Simulation . . . . . . . . . . . . . . . . . . . 151

6.3. High Injection Rate Simulation . . . . . . . . . . . . . . . . 157

6.4. $45^{\circ}$ Injection Simulation . . . . . . . . . . . . . . . . . . . 163

6.5. $0^{\circ}$ Injection Simulation . . . . . . . . . . . . . . . . . . 169

6.6. Conclusions . . . . . . . . . . . . . . . . . . 175

$\begin{array}{lr}\text { 7. Conclusions and Further Work } & 177\end{array}$

Appendix A. Ionisation Calculations for $\mathbf{H}_{2} \quad \mathbf{1 8 2}$

Appendix A.0.1. Rate of decay of $\mathrm{H}_{2}$. . . . . . . . . . . . . . . 183 
Appendix A.0.2. Rate of production and decay of $\mathrm{H}_{2}^{+} \ldots$. . . . . . 183

Appendix A.0.3. Rate of production and decay of $\mathrm{H}$. . . . . . . . . 183

Appendix A.0.4. Rate of production of $\mathrm{H}^{+} \ldots$. . . . . . . . . . 184

Appendix A.0.5. Rate of production of electrons . . . . . . . . . . . 184

$\begin{array}{lr}\text { Appendix B. Ionisation Calculations for } \mathrm{N}_{2} & \mathbf{1 8 6}\end{array}$

Appendix B.0.6. Rate of Decay of $\mathrm{N}_{2}$. . . . . . . . . . . . . . . 186

Appendix B.0.7. Rate of production and decay of $\mathrm{N}_{2}^{+} \ldots \ldots . . .186$

Appendix B.0.8. Rate of production and ionisation of N . . . . . . 187

Appendix B.0.9. Rate of production of $\mathrm{N}^{+} \ldots \ldots . \ldots 188$

$\begin{array}{lr}\text { References } & 189\end{array}$ 


\section{Introduction}

The solar wind is a continuous stream of ions and electrons which are emitted from the Sun's outer corona. These ions are accelerated as they travel outward until it becomes a supersonic flow of plasma. The interplanetary magnetic field (IMF) is embedded in the solar wind due to its very high conductivity and is carried outwards along with it, in the limit of infinite conductivity the magnetic field lines move with the fluid flow in the so-called 'frozen-in' approximation. The solar wind with its frozen-in magnetic field fills the solar system and defines the boundaries of the heliosphere and interacts with many different types of celestial object along the way. If the interaction is with a magnetised body then the solar wind will be deflected due to the inability of the separate magnetic fields to mix and, if the body's magnetic field is sufficiently powerful, can form a magnetosphere which shields any neutral atmosphere/exosphere it may have from the effects of the solar wind. This is a typical interaction of a magnetised planet with an atmosphere and the solar wind. If the object is unmagnetised and has no significant atmosphere, such as most asteroids, then the solar wind will impact its surface and ionise particles there which are subsequently picked up by the solar wind flow as pickup ions pickup ions are very common in various situations throughout the heliosphere. It should be mentioned that there are, of course, pickup electrons associated with these processes of ionisation but as these make a much smaller contribution to the energy and momentum budget of the plasma due to their lower mass they are normally neglected as being unimportant aside from charge neutrality considerations. If the object does have a significant atmosphere but no intrinsic magnetic field then it is possible for this interaction to induce a magnetosphere. This is the case for Venus and also for comets with a sufficiently large production rate of neutrals close enough to the Sun, the comets can generate a cometosphere as the neutrals escape the weak gravitational attraction of the comet and become ionised, often found at large distances from the comet's nucleus. Once ionisation of neutrals occurs in any of these situations then it marks the beginning of plasma interactions.

Spacecraft observations have shown us so much about the processes involved in the physics of space plasmas and more and a key element of any successful mission is 
orbital and attitude control which relies on the usage of thrusters. Most commonly, chemical thrusters are used for this which produce a plume of neutrals, these neutrals are then ionised and picked up by the solar wind and although these are 'artificial' pickup ions they have the same underlying physics as for naturally occurring pickup ions.

The basis and motivation for the work in this thesis is the NASA Solar Probe Plus (SPP) mission. Solar Probe Plus is due to be launched in 2018 and will eventually approach to within ten solar radii of the sun's photosphere. Solar Probe Plus will obtain the first in-situ measurements of the near-solar environment, the data from which is expected to drastically enhance our knowledge and understanding of many hotly debated and open questions in heliophysics such as coronal heating and the acceleration and evolution of the solar wind. Furthermore, it is hoped that such direct measurements of the solar wind will contribute fundamentally to our understanding of and ability to predict the radiation environment in which future manned missions will have to operate. Additionally SPP will aid in determining the dynamics and structure of the formation of the interplanetary magnetic field at the source of the solar wind and will have the opportunity to explore the near-solar dusty plasmas and their influence on the solar wind and formation of energetic particles.

Solar Probe Plus is planned to use repeated gravity assists at Venus in order to slowly lower its perihelion over several years until it reaches its closest approach of $\approx 6,000,000 \mathrm{~km}$. Due to making such a close approach to the Sun the radiation it experiences will reach over 500 times the intensity of Earth orbit. Due to this SPP will have a reinforced carbon-carbon composite solar shield behind which all of the sensors and scientific instruments will shelter. Due to this incredibly demanding pointing requirement of SPP attitude control is critical during the perihelion transition and thus the spacecraft will have to perform either frequent small thruster firings in order to keep the body of the spacecraft in the heat shield's shadow or else will have to use reaction wheels to 'store' the momentum and then perform a large de-spinning thrust. It is to these manoeuvres that the body of this work holds the most relevance. As the probe passes the Sun at its closest approach it will reach speeds of $\approx 200 \mathrm{kms}^{-1}$ at which time it will become the fastest man-made object ever, a record currently held by Helios II at $\approx 70 \mathrm{kms}^{-1}$. A second motivation for this work is the opportunity to use the thruster firings of SPP as an active probe of pickup ion processes in the solar wind plasma. The thrusters will produce significant amounts of neutral particles which will become ionised in the high intensity radiation environment and will then quickly couple to the solar wind plasma and become a source of free energy which could drive instabilities and produce electromagnetic 


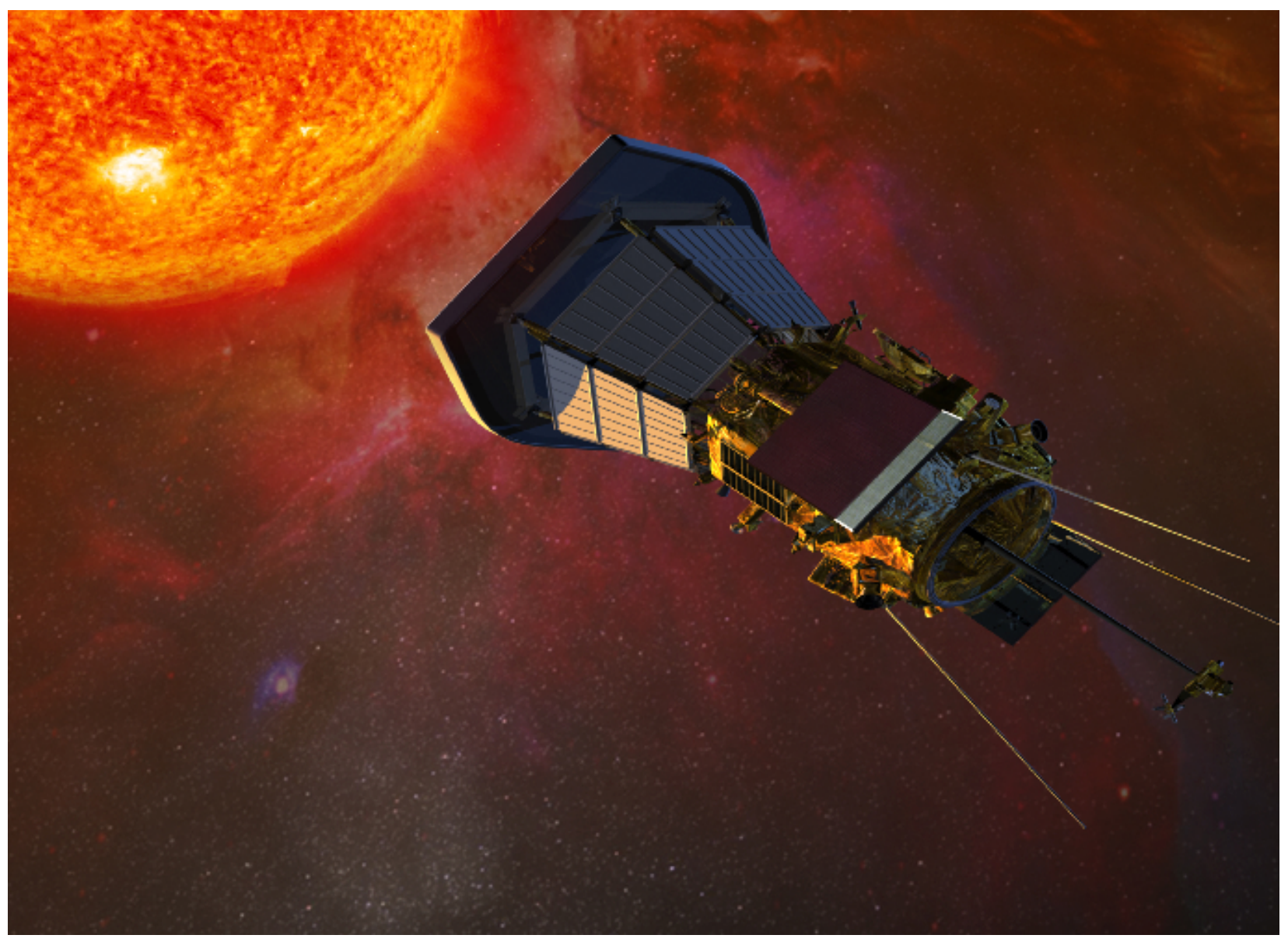

Figure 1.1.: An artist's impression of Solar Probe Plus as it gathers data on its way to the Sun with its solar panels folded in. Credit: JHU/APL.

wave activity. Even in the absence of instabilities it is expected that these injections of additional mass into the solar wind flow will still have a noticeable effect at least due to mass loading processes.

The goal of this work is to simulate the operation of a generic thruster and its interaction with the solar wind. The expansion of the neutral plume produced by a thruster firing is modelled separately to the plasma interactions, the neutral model calculates the ionisation of the plume and provides the rate at which ions need to be injected into the hybrid plasma simulation. Simulations were performed in different conditions, i.e. for both high and low injection rates with respect to a reference and for different angles of injection with respect to the magnetic field. The injection rate of a simulation corresponds to the length of a thruster burn or, equally, to the total amount of mass burned in a single thrust as a longer burn results in a higher mass, larger neutral cloud. This was done in order to investigate the effects the thruster firing has on the ambient solar wind and how long any perturbations will last as 
well as the extent of the region in which they are important.

Current research surrounding spacecraft thrusters falls largely into one of two categories: electric and chemical thrusters. Electric thruster research is largely concerned with the near-field effects of the ions injected into the solar wind or on the charging of the spacecraft. Other topics covered by the literature include wake formation behind the spacecraft, neutralisation of the ion beam and waves or instabilities produced as a result of the addition of new ions. Since this type of investigation is often concerned with individual particle kinetics particle in cell (PIC) codes are prevalently used for simulations which precludes large simulation domains and long duration simulations due to computational restraints which means that often little thought is given to any large scale effects that may occur. Electrostatic ion thrusters, which are a type of electric thruster, ionise their propellent by electron bombardment and then accelerate and focus the resulting plasma beam to provide directed thrust. The beam is neutralised by directly emitting a stream of electrons into it from a neutraliser specifically for this task. Electric thrusters typically will have a lower thrust than an 'equivalent' chemical thruster but they have a much higher specific impulse which makes them more mass-efficient and they are able to operate continuously for long periods of time. Deep Space 1 was the first craft to use such an engine (NSTAR ion engine) for their primary propulsion system for an interplanetary mission although various other craft use electric propulsion for station keeping manoeuvres.

Chemical thruster research tends to focus on only the neutral aspect of the thruster firing, that is, topics such as the near thruster flow-field, the propagation of the neutrals and the shape of the plume produced but this is often for specific thruster models. Effects of the neutrals on the spacecraft are also investigated such as force loading from the firing itself as well as any thermal loading which might occur, backflow of the neutrals and any deposition on the spacecraft which might result from this with particular focus on the solar arrays and any exposed sensors. Plume impingement and sputtering of the spacecraft itself are also topics of interest although there is little consideration given for the effects of chemical thrusters within a plasma setting. In addition to this, research into the interaction of ions with the solar wind, whilst diverse in topic, does have some gaps when it comes to the entire 'life cycle' of a thruster firing. Topics which are most considered in the context of solar wind interaction are pickup ions in various contexts such as throughout the heliosphere, in the vicinity of planets and their moons, around comets of varying sizes and at different solar distances and instabilities associated with these processes.

A simulation code was developed in order to model the evolution and propagation 
of the neutral thruster plume. This model also includes dynamic ionisation the neutral plume over the duration of the simulation and produces injection rates which are used as an input to a hybrid plasma code. The hybrid plasma code used, HYPSI, is fully self consistent and utilises the injection rates produced by the neutral model in order to 'inject' additional ions into the simulation over time according to the rates of ionisation of the species considered. A hybrid code was chosen as it allows the study of ion kinetic effects on the scale of ion gyration and their interaction with the solar wind without requiring the much larger computational requirements of a full PIC code which also models electrons kinetically. The evolution of these particles and the effect they have on the ambient solar wind environment is then determined as the simulation progresses.

An initial investigation was performed along with a modest parameter survey and an introductory analysis of ion kinetics in order to characterise the types of effects which may be seen and how they might vary with solar conditions as well as to determine both the spatial and temporal extent of any disturbances generated as a result of a thruster firing. It was found that as the solar wind accommodates the newly injected ions that a bow wave forms, as more mass is loaded onto the solar wind an interaction region is induced which then expands over time, this has both magnetic and density components at its edge. Further to this the magnetic field displays draping patterns around this region, inside of which the spacecraft remains, in a similar manner to that found at comets. As this develops a region of magnetic flux pileup often accumulates ahead of the spacecraft. These effects were found to vary with both the injection angle with respect to the magnetic field and with the injection rate, or equivalently, the size of the thruster firing. Which of these has the largest effect depends upon the feature in question, often the amount of mass loaded onto the solar wind has the largest effect but the injection angle can also have a significant effect on the interactions which occur. Surprisingly there was no evidence found of instabilities of the type which are commonly found in cometary environments despite the similarity of the initial velocity distributions, such as the electromagnetic ion cyclotron (EMIC) instability or the mirror mode instability. Whilst the kinetic effects were largely expected in that, in the solar wind frame, the injected ions were slowed down and the solar wind was accelerated there was also evidence that injected ions have the potential to also be significantly accelerated if the conditions are favourable.

Chapter 2 outlines the physics of pickup ions in various settings from planetary environments to cometospheres and to their behaviour throughout the heliosphere. Chapter 3 discusses the details of the simulation code developed to model the neutral 
thruster plume and its interactions with the hybrid code. Chapter 4 presents the first results of the hybrid simulation, the main effects observed and the interaction of the thruster plume with the solar wind. Chapter 5 develops this picture further by performing a parameter survey in order to gain some understanding of how the influence of the thruster plume on the solar wind will change as the solar wind conditions change. Chapter 6 will present the results of particle tracing and the main types of particle motion observed and explanations for their trajectories. Finally the main conclusions of the work will be summarised in Chapter 7 . 


\section{The Physics of Pickup Ions}

The following chapter will discuss and review the physics of pickup ions (PUIs). The interaction of newborn ions from a thruster firing with the solar wind is an example of a pickup ion coupling process. Therefore in this chapter I review the processes of ion pickup and the various heliospheric environments in which they occur such as near to comets, planets and in active release experiments.

\subsection{Introduction}

The solar wind flow is assumed to be a fully ionised and highly conducting plasma. The magnetic field will be frozen to the solar wind flow due to this high conductivity. Even in this flow there are some few neutral particles present, these particles will eventually become ionised as a result of one of various processes such as photoionisation or charge exchange. In the solar wind frame (where the motional electric field is zero), these newborn ions are injected at a different velocity to the solar wind, $\vec{V}_{b}$. They then gyrate about a stationary magnetic field line with a velocity of $\vec{V}_{b} \sin (\theta)$ where $\theta$ is the angle between the PUI's velocity and the magnetic field vectors and stream along the field line with a velocity of $\vec{V}_{b} \cos (\theta)$ in a frame stationary with respect to the Sun. Since the magnetic field lines are not stationary, injected particles will therefore feel a convective electric field:

$$
\vec{E}=-\vec{V}_{s w} \times \vec{B}
$$

Thus in this frame these new ions will be accelerated along the electric field whilst gyrating about the magnetic field and thus describing cycloidal motion in space. Generally the particles will stream along $\vec{B}$ with a parallel velocity of:

$$
V_{\|}=\left(\vec{V}_{s w}-\vec{V}_{b}\right) \cdot\left(\frac{\vec{B}}{|\vec{B}|}\right)
$$


and a perpendicular velocity of:

$$
V_{\perp}=\left(\vec{V}_{s w}-\vec{V}_{b}\right) \times\left(\frac{\vec{B}}{|\vec{B}|}\right)
$$

Therefore the ions will be accelerated perpendicular to $\vec{B}$ until their average velocity reaches the solar wind flow velocity, it is by this acceleration that the particles are 'picked up' by the solar wind flow. This guiding centre velocity is:

$$
\vec{V}_{g c}=\frac{\vec{E} \times \vec{B}}{B^{2}}
$$

The equation of motion of a newborn pickup ion is solved by equating the Lorentz force and Newton's second law to get:

$$
\dot{\vec{V}}=\frac{q}{m}(\vec{V} \times \vec{B}+\vec{E})
$$

If we choose a geometry such that $\vec{B}=B_{z}$ then, using ideal MHD, the motional electric field will be:

$$
\begin{aligned}
\vec{E} & =-\vec{V}_{s w} \times \vec{B} \\
\vec{E} & =-\left|\begin{array}{ccc}
\hat{x} & \hat{y} & \hat{z} \\
V_{s w, x} & V_{s w, y} & V_{s w, z} \\
0 & 0 & B_{z}
\end{array}\right| \\
& =-V_{s w, y} B_{z} \hat{x}+V_{s w, x} B_{z} \hat{y}
\end{aligned}
$$

Which gives:

$$
\begin{aligned}
\dot{V}_{x} & =\frac{q}{m}\left[V_{y} B_{z}+E_{x}\right] \\
& =\omega_{c}\left[V_{y}+\frac{E_{x}}{B_{z}}\right]
\end{aligned}
$$

and

$$
\begin{aligned}
\dot{V}_{y} & =\frac{q}{m}\left[-V_{x} B_{z}+E_{y}\right] \\
& =-\omega_{c}\left[V_{x}-\frac{E_{y}}{B_{z}}\right]
\end{aligned}
$$


with

$$
\dot{V}_{z}=0
$$

where $\omega_{c}=\frac{q B_{z}}{m}$.

Therefore:

$$
\ddot{V}_{x}=-\omega_{c}^{2}\left[V_{x}-\frac{E_{y}}{B_{z}}\right]
$$

and

$$
\ddot{V}_{y}=-\omega_{c}^{2}\left[V_{y}+\frac{E_{x}}{B_{z}}\right]
$$

the solutions to which are:

$$
V_{x}(t)=A \sin \left(\omega_{c} t+\gamma\right)+\frac{E_{y}}{B_{z}}
$$

and

$$
V_{y}(t)=A \cos \left(\omega_{c} t+\gamma\right)-\frac{E_{x}}{B_{z}}
$$

with

$$
V_{z}(t)=V_{z}(0)
$$

where $A$ and $\gamma$ are constants which depend upon the initial conditions and phase of the pickup ion and solar wind motion. Due to the motion being cycloidal the particles will have a varying velocity and hence energy, the maximum values of which are:

$$
\begin{gathered}
V_{\text {max }}=2 V_{s w} \sin (\alpha) \\
E_{\text {max }}=2 m_{i} V_{s w}^{2} \sin ^{2}(\alpha)
\end{gathered}
$$

Where $\alpha$ is the angle between the solar wind flow direction and the magnetic field: the particle's pitch angle. This is also the reason for the $2 V_{s w}$ cutoff in the velocity distribution of pickup ions.

In velocity space cycloidal motion corresponds to a ring/ring-beam velocity distribution. Figure 2.1 schematically displays the scattering of ions from a ring distribution into a simple shell distribution in both the spacecraft frame and the solar wind frame [Szegö et al., 2000]. Such distributions are unstable and hence will cause the production of plasma waves, these waves cause the particles to scatter in both pitch angle and, eventually, energy. Thus, over time, these distributions will become spherical and isotropic as energy is transferred from the solar wind to the newborn ions and the waves are produced.

The pitch angle scattering process was predicted first by Gaffey et al. [1988] 

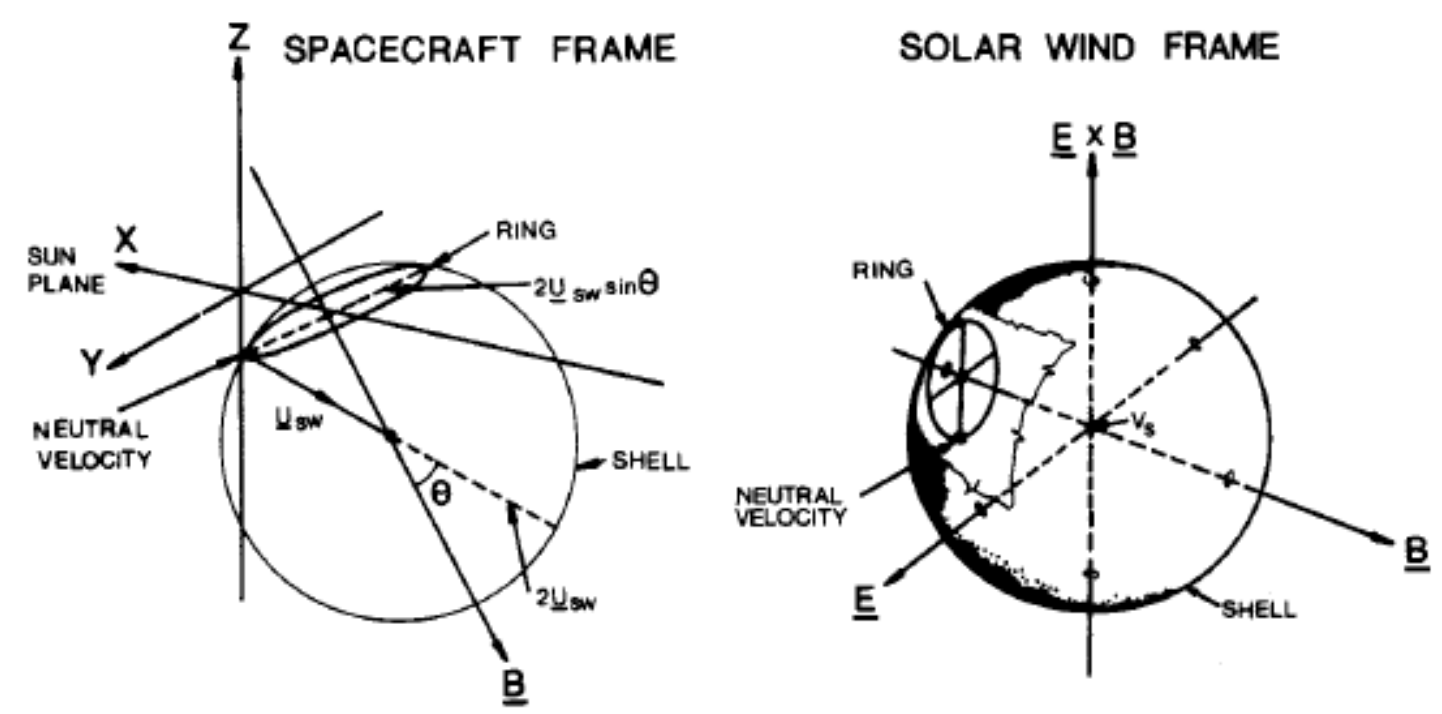

Figure 2.1.: Schematic view of the pickup process in the spacecraft frame (left) and the solar wind frame (right). Ions are initially in a ring velocity before becoming scattered into a simple shell distribution. Taken from [Szegö et al., 2000].

as the simple shell model and then developed to a bispherical scattering model by Galeev and Sagdeev [1988] and Miller et al. [1991a]. The simple shell approximation of pitch angle scattering assumes that the initial velocity of the neutral particles before ionisation is a point in velocity space, that is, they have a bulk velocity of $V_{b}$ with negligible spread. Also, for simplicity, it is assumed that the magnetic field is uniform and that the solar wind flow is homogeneous and has a constant velocity, $V_{s w}$. The ions are therefore injected with this speed and quickly evolve their velocity distribution from a point into a ring-like distribution as a result of gyration about the magnetic field lines.

As this type of (non-Maxwellian) ring distribution is unstable, waves will be produced which use the anisotropy in the newborn ion energy as its source of free energy to drive the instability [Wu and Davidson, 1972]. The progression of this instability, in the simple limit, will cause the energy distribution of the new ions about the mean to become more isotropic and hence they cause pitch angle scattering of these ions which leads to a distribution in velocity space which resembles a spherical shell.

A more realistic mechanism of particle scattering is the bispherical distribution put forth by Galeev and Sagdeev [1988]. In this model rather than filling a single thin shell in velocity space the particles instead fill parts of two shells centred on $\pm V_{A}$ along the axis of the ambient magnetic field in the solar wind frame. This corresponds to the velocity of upstream and downstream propagating waves and 
can occur when waves are seen to travel in opposite directions. Thus ions are scattered backward by forward travelling waves and are scattered forward by backward travelling waves into a bispherical velocity space distribution. Particles usually lose energy in this frame to the waves they drive and hence their velocity will decrease as they are scattered in pitch angle, however, in the wave frame the particle motion conserves energy. Figure 2.2 shows a bispherical velocity distribution, the paths connecting points $\mathrm{A}$ and $\mathrm{D}$ as well as $\mathrm{B}$ and $\mathrm{C}$ are the two possible routes of scattering but since being scattered towards $\mathrm{A}$ or $\mathrm{C}$ would result in an increase in energy and the particles must, on average, lose energy in this plot the path between $\mathrm{B}$ and $\mathrm{D}$ is the route the majority of the scattering follows [Coates, 2004]. The bispherical distribution is composed of two spherical shells with centres focussed on the forward and backward wave phase speeds. A dispersive analysis of bispherical scattering was performed by Isenberg and Lee [1996] and shows that dispersive effects can be important when modelling the quasi-linear interactions of pickup ions with their self-generated waves.

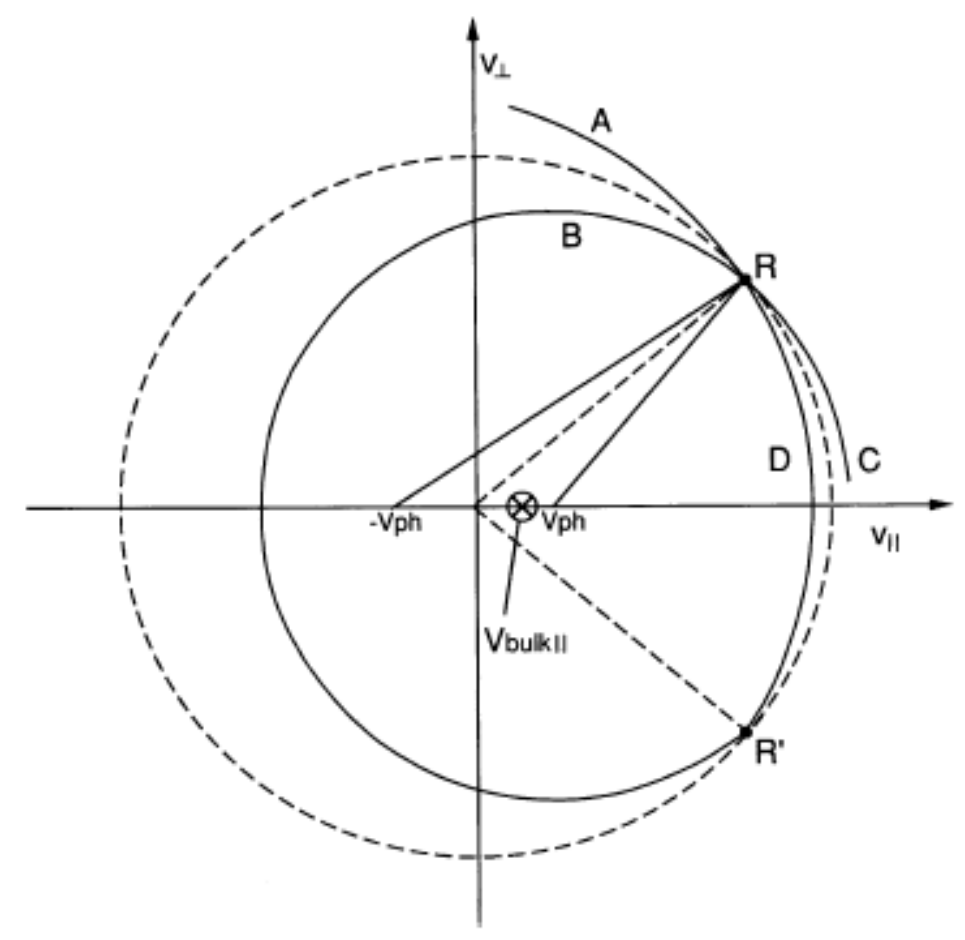

Figure 2.2.: Schematic view of the bispherical velocity distribution. Taken from [Coates, 2004].

Due to pickup ions merely being newborn ions introduced into a plasma they can be found quite commonly throughout the solar system anywhere that neutral particles or ions are being ejected into space. The interstellar medium (ISM) has a 
neutral and a plasma component, the plasma part interacts with the solar wind to form the boundaries of the heliosphere: the heliopause, which separates the interstellar and solar magnetic fields, the termination shock where the solar wind speed decreases from supersonic to subsonic and possibly a heliospheric bow shock/wave in the interstellar medium propagating ahead of the heliopause. PUIs can be seen throughout the heliosphere as neutrals enter the heliosphere primarily from the interstellar medium and are ionised, primarily by charge exchange, before being picked up and swept outward along with the solar wind flow [Kallenbach et al., 2000]. They can be seen in more impressive locations such as in the region of comets where the activity depends both on the distance from the comet's nucleus and on the comet's distance from the Sun [Cravens and Gombosi, 2004]. In these cases there can be significant mass loaded onto the solar wind flow and the density of these pickup ions is always greater near to a comet than those from the interstellar medium throughout the 'undisturbed' heliosphere and therefore the effects are less subtle and more readily observable.

Pickup ion activity can also be seen closer to home in such places as the Earth's bow shock and magnetosphere [Mall et al., 1998, Sadovski and Skalsky, 2014, Zhang et al., 2001] and also those of other planets such as Mars [Cowee et al., 2012, Cravens et al., 2002, Wei and Russell, 2006] and Mercury [Baumjohann et al., 2006, Koehn et al., 2002, Sarantos et al., 2009] although to a lesser extent. Additionally certain moons in the solar system can be sources of pickup ions such as Io at Jupiter [BlancoCano et al., 2001, Russell et al., 1998] and Enceladus at Saturn [Sittler et al., 2004, Tokar et al., 2008, Tseng et al., 2008]. In the case of Io, it is volcanically active and ejects an estimated 1.5 tons per second of sulphur and oxygen based molecules into space which eventually form a torus about Jupiter [Dols et al., 2012]. For Enceladus, it is geologically active and has liquid water under its crust which occasionally results in jets of water group molecules being ejected, these then form the torus of the $\mathrm{E}$ ring which is composed of micron sized water-ice grains where they are then ionised and picked up by Saturn's magnetic field.

Finally some pickup ions are created artificially by events such as spacecraft thruster firings, sputtering of space debris or even active release experiments such as AMPTE where large quantities of neutral material is released in a small volume over a very short time period [Bernhardt et al., 1987, Gurnett, 1985, Häusler et al., 1986, Roeder et al., 1987]. These man-made events can have a significant impact on the flow of the solar wind in the short to medium term. If the mass introduced is large enough then the solar wind can have trouble accommodating the new mass quickly enough and will then form bow-waves or even shocks in extreme cases. 
Pickup ions in the heliosphere can be identified by three broad properties: charge, spatial distribution and their velocity distribution [Neugebauer, 2001]. Heavy ions found naturally in the solar wind tend to be highly charged due to the high temperatures experienced in the solar corona. Pickup versions of such heavy ions however are generally singly charged as they are ionised in regions of higher solar wind and EUV flux and then are swept outward by the convective flow before further ionisation has the opportunity to take place. Thus this is a relatively simple way to distinguish between the singly charged pickup ion population and the multiply charged solar wind heavy ions.

Ions produced from interstellar neutrals have a specific spatial number density distribution throughout the heliosphere which depends on the angle between the interstellar wind flow direction and the observer's position around the sun as well as on the ionisation rate of the neutrals. From this the distance from the Sun at which the maximum abundance of pickup ions will be found can be calculated. In contrast to this, ions with a local origin such as comets or ions from planetary environments have a number density which decreases with the distance from the source of these ions.

In the heliosphere pickup ions are initially produced with a velocity much lower than the solar wind velocity from relatively slow moving neutrals. These ions are then rapidly convected outward where fluctuations in the magnetic field and pitch angle scattering act to broaden the distribution into a shell in velocity space. The ions are then accelerated by the convective electric field depending on the angle between the solar wind flow and magnetic field such that some ions are able to obtain velocities greater than that of the solar wind. Due to these, and other, processes the pickup ion velocity distribution in the solar wind frame is relatively flat with a steep cut off around $2 V_{s w}$, see also equation 2.14 [G. Gloeckler et al., 2004].

The study of pickup ions can explain the origin of instabilities and waves observed both in the solar wind and in planetary environments by determining their velocity distributions and performing a stability analysis to investigate how certain distributions are unstable. Additionally an investigation into the velocity and spatial distributions of identified pickup ion populations can provide information on the source of the ions and hence the neutrals such as relative abundances of particular ion species in the interstellar medium. Further, PUIs can be responsible for atmospheric 'scavenging' primarily around non-magnetic planets. This is where the PUIs encounter the atmosphere or surface of a planet and cause impact ionisation which produces even more pickup ions at the expense of the atmospheres of these 


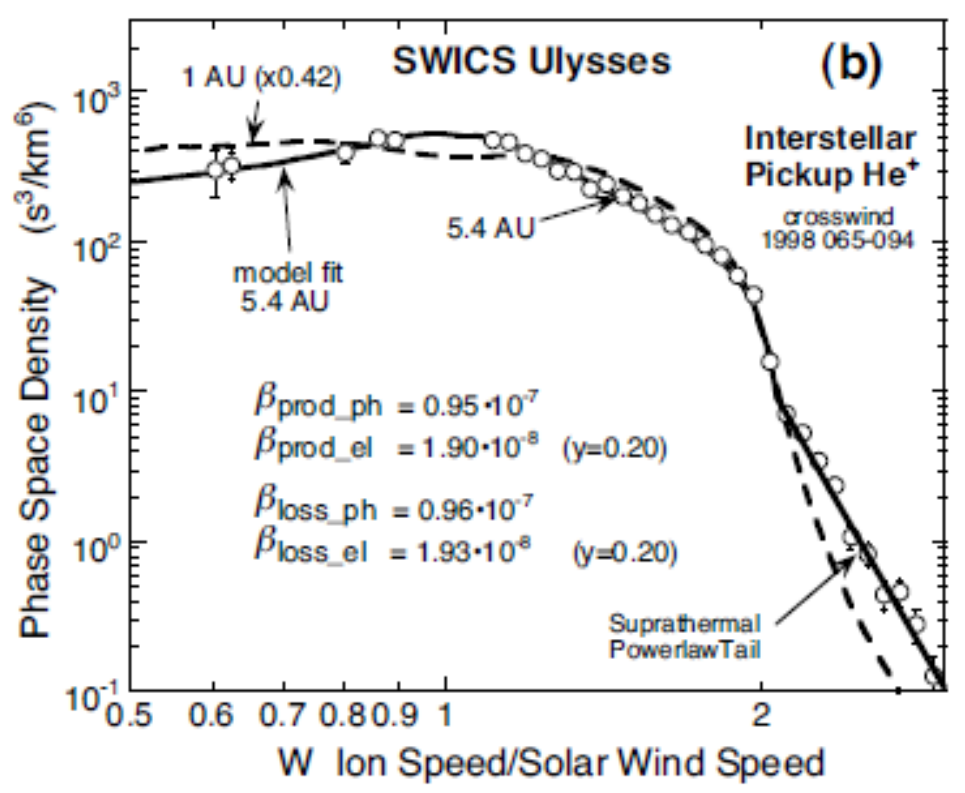

Figure 2.3.: SWICS data from Ulysses showing the phase space drop-off at $2 V_{s w}$. Taken from [G. Gloeckler et al., 2004].

planets. In the absence of a replenishing mechanism the atmospheres of the planets can become slowly diminished over time.

\subsection{Interstellar Pickup Ions}

The following section will discuss the occurrence and behaviour of pickup ions throughout the heliosphere, including the source of neutral particles which then become the pickup ions, filtration of interstellar neutrals at the termination shock, the helium focusing cone and various situations in which the pickup ions can be accelerated.

\subsubsection{Source of Neutral Particles}

The dominant pickup ion population in the heliosphere is a result of the motion of the Sun sweeping interstellar neutral particles into the heliosphere where they are ionised by primarily charge exchange, photo-ionisation and electron/proton impact before being picked up by the solar wind. These interstellar neutrals are swept in from the local interstellar cloud with a velocity, due to the relative motion of the Sun, of $\approx 20-25 \mathrm{kms}^{-1}$ [Kallenbach et al., 2000]. These neutral particles can penetrate the heliosphere to within several AU of the Sun before it becomes mostly ionised 
and it is once more swept towards the termination shock by the solar wind flow. Pickup ions are produced from these neutrals which are moving relatively slowly compared to the solar wind. They are almost all singly charged and the eventual result of pickup is that neutrals with only a few eV of energy are accelerated as ions to energies reaching several keV.

This process is, of course, strongest closer to the Sun and it therefore provides a structure to the distribution of both the neutrals and the resulting pickup ions throughout the heliosphere. The maximum theoretical velocity for an ion picked up by the solar wind is $2 V_{s w}$ as it gyrates about a magnetic field line which is itself being convected at the solar wind flow speed and yet observations have been made of pickup ions with speeds higher than this in situations such as downstream of shocks. This is evidence that pickup ions and other supra-thermal ion populations can be preferentially, or more efficiently, accelerated by certain processes.

The following pickup ions were discovered from the data provided by SWICS on the Ulysses craft: ${ }^{1} \mathrm{H}^{+},{ }^{4} \mathrm{He}^{+},{ }^{3} \mathrm{He}^{+},{ }^{4} \mathrm{He}^{++}, \mathrm{N}^{+}, \mathrm{O}^{+}$and $\mathrm{Ne}^{+}$[Kallenbach et al., 2000]. Based on the way their abundances vary with solar distance and latitude as well as the shape of their velocity distributions it was determined that most of these pickup ions originated from the neutral interstellar gas entering the solar system.

This said, Ulysses also found evidence of $\mathrm{C}^{+}$pickup ions at all solar distances and latitudes but with vastly different spatial distributions and phase-space densities compared to those known to be as a result of the interstellar gas [Gloeckler and Geiss, 1998].

When pickup ions are born they have an almost isotropic velocity distribution in the range of $0-2 V_{s w}$ with a sharp drop-off at $2 V_{s w}$ as shown in fig 2.3. This drop-off is a result of the maximum velocity a pickup ion can have although it is not exact and some particles can have more energy and some can have less. As these ions are swept outwards they undergo adiabatic cooling and thus their velocity distributions develop a narrow peak at the solar wind bulk speed.

From the narrowness of a velocity distribution it can be deduced roughly where that particular pickup ion population originated from. It was this technique which allowed the identification of a source of pickup ions which originated within a few $\mathrm{AU}$ of the Sun by a narrow peak in the velocity distribution centred around $V_{s w}$ embedded in the more isotropic distribution of the interstellar pickup ion population with the characteristic drop-off at $2 V_{s w}$ which indicated that the isotropic population was produced locally [Gloeckler and Geiss, 1998]. This was later termed the 'Inner Source' and is thought to be produced largely by interstellar grains that have managed to penetrate into the inner heliosphere at higher solar latitudes and then 
sublimate molecules which become ionised and are then picked up.

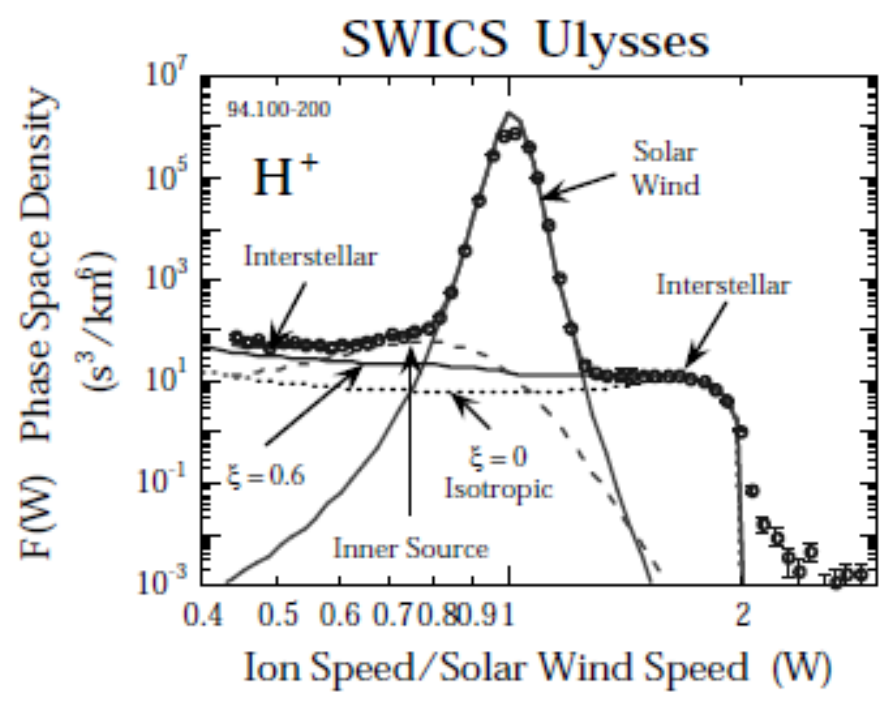

Figure 2.4.: Phase space density of $\mathrm{H}^{+}$verses the ion speed in the spacecraft frame compared to the solar wind speed using SWICS data. The inner source ions can be seen as a peak centred on $V_{s w}$. The $2 V_{s w}$ drop-off is also visible. Taken from [Gloeckler and Geiss, 1998].

\subsubsection{Filtration and Other Effects}

The neutral particles that comprise the local interstellar cloud undergo a process known as 'filtration' in the corresponding filtration region as they enter the heliosphere through the termination shock [Gloeckler and Geiss, 1998, Kallenbach et al., 2000, Linde and Gombosi, 2000, V. Izmodenov et al., 2004, Zank et al., 1999]. This filtration region is thought to be around 100-200 AU wide and lies beyond the heliopause forming a boundary which separates the heliospheric plasma from the interstellar plasma. As the interstellar plasma is forced to move around the heliosphere it is slowed down and, by charge exchange, causes some hydrogen atoms to become fast protons.

This process also affects other atoms and molecules that have a high charge exchange cross section including $\mathrm{O}$ atoms. They collide with the hot, slow protons present, become ionised and therefore do not make it into the heliosphere as the interstellar magnetic field then sweeps these ions around the heliosphere. Helium atoms, however, are largely unaffected by this process and hence from their properties some information on the kinetic parameters of the local interstellar cloud can be gained. 
Interstellar heavy atoms which are swept into the heliosphere as part of the 'interstellar breeze' are affected by the Sun's gravitational field and are thereby focused into a conical region on the opposite side of the Sun from which they entered the heliosphere called the gravitational focusing cone [Chalov, 2014, G. Gloeckler et al., 2004, Gershman et al., 2013]. Due to helium having a very low ionisation efficiency it is able to penetrate deeply into the heliosphere and the population has not been significantly depleted even by $1 \mathrm{AU}$ and therefore $\mathrm{He}^{+}$pickup ions have the highest density in the regions downwind of the focusing cone.

The focusing cone was first detected by EUV $58.4 \mathrm{~nm}$ backscattering observations made by Weller and Meier [1974] and is shown schematically in Figure 2.5. Interstellar $\mathrm{He}^{+}$pickup ions were first found in this region of space in 1984 by SULEICA of the AMPTE mission. The focusing cone was investigated in more detail later on by the ACE spacecraft which was at the L1 point. The shape of the focusing cone, and the number density of the $\mathrm{He}^{+}$pickup ions therein, depends on parameters in interstellar space that control the heliospheric distribution of the interstellar helium within the inner heliosphere and also upon the ionisation rate of helium.

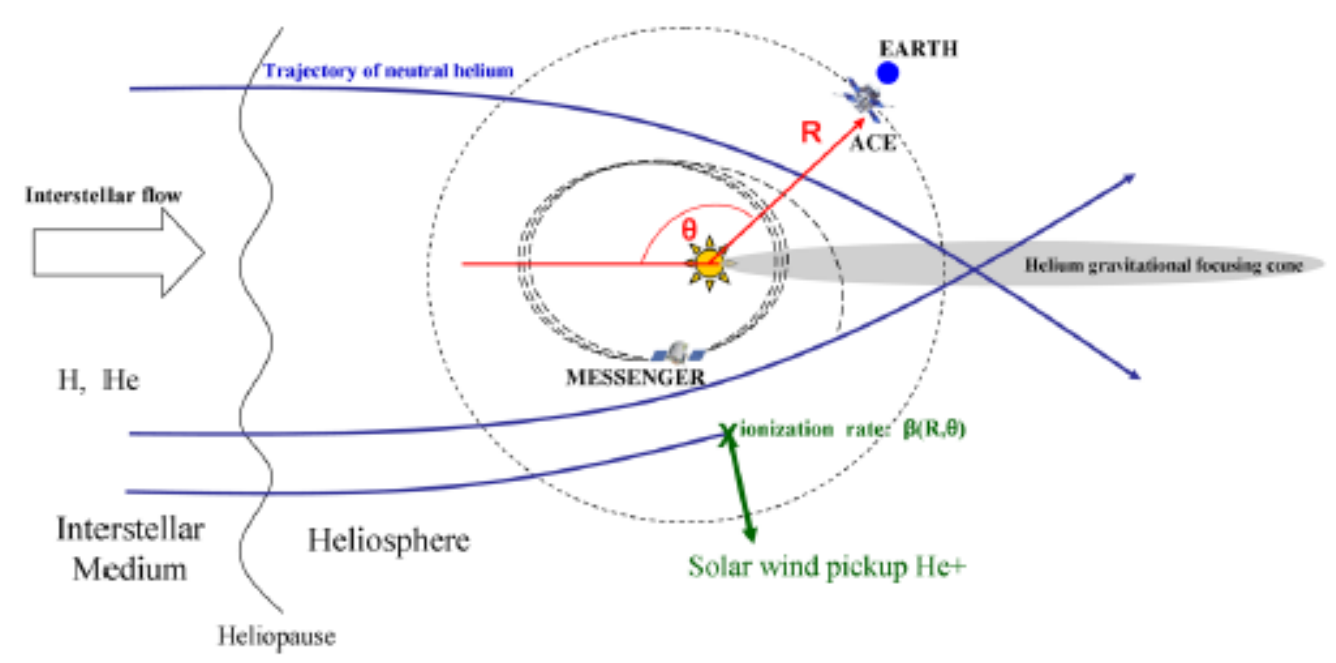

Figure 2.5.: Schematic view of neutral particle trajectories which converge to form the gravitational focusing cone downwind of the interstellar flow. Taken from [Gershman et al., 2013].

Since the helium passes to within 0.5 AU before the majority of it ionises means that a spacecraft at $1 \mathrm{AU}$ will be well positioned to sample the distribution of the helium present in the focusing cone. 


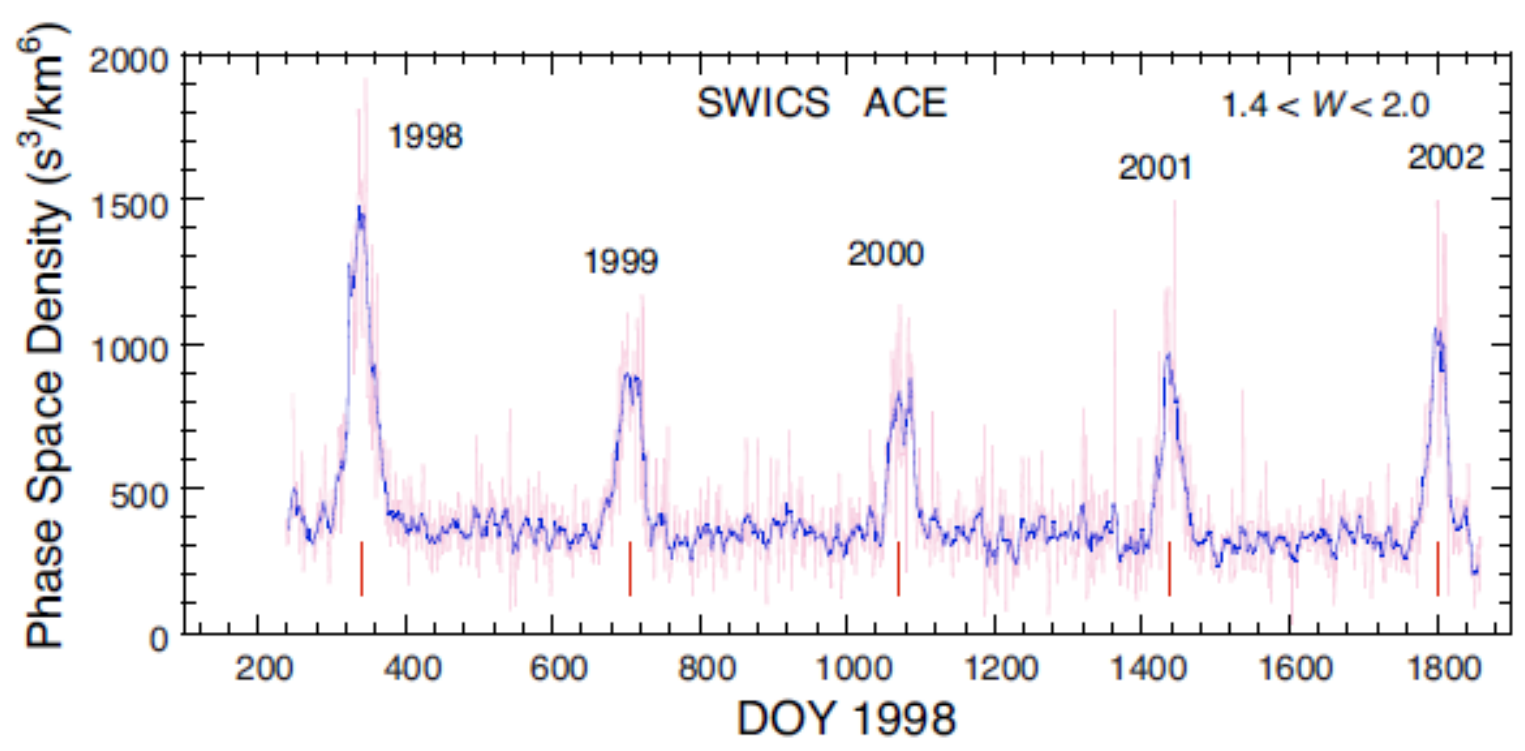

Figure 2.6.: Passages of the interstellar helium focusing cone from 1998 to 2002 as observed using PUIs by ACE/SWICS. Graph shows the phase space density of $\mathrm{He}^{+}$PUIs. Taken from [G. Gloeckler et al., 2004].

\subsubsection{Anomalous Cosmic Rays}

In interplanetary space it was known that there were two distinct populations of energetic particles; solar energetic particles which originate in events on the Sun and galactic cosmic rays (GCRs) which are produced by sources outside of the solar system. A third component was discovered by Pioneer10, IMP5 and IMP7 which represents a sample of the nearby interstellar medium which has penetrated the heliosphere.

These so-called anomalous cosmic rays (ACRs) have an origin which begins with interstellar neutrals entering the heliosphere and falling inward toward the Sun until such time as they are ionised. Once ionised these particles are picked up by the convected magnetic field of the Sun carried outward by the solar wind flow. The picked up ions are swept towards the outer heliosphere in this way and accelerated to the solar wind velocity on average.

It is theorised that these pickup ions are accelerated further within the termination shock and a small percentage of those can diffuse out and back into the heliosphere where they are able to stream back into the inner heliosphere and potentially interact with, or be captured by, the Earth's magnetosphere [Gloeckler and Geiss, 2001].

These ACRs are so-called due to both their energy of 10-100 MeV being intermediate between the SEPs and the traditional GCRs. Additionally their charge state is often close to +1 whereas GCRs can be multiply charged [Panasyuk, 2001]. A 

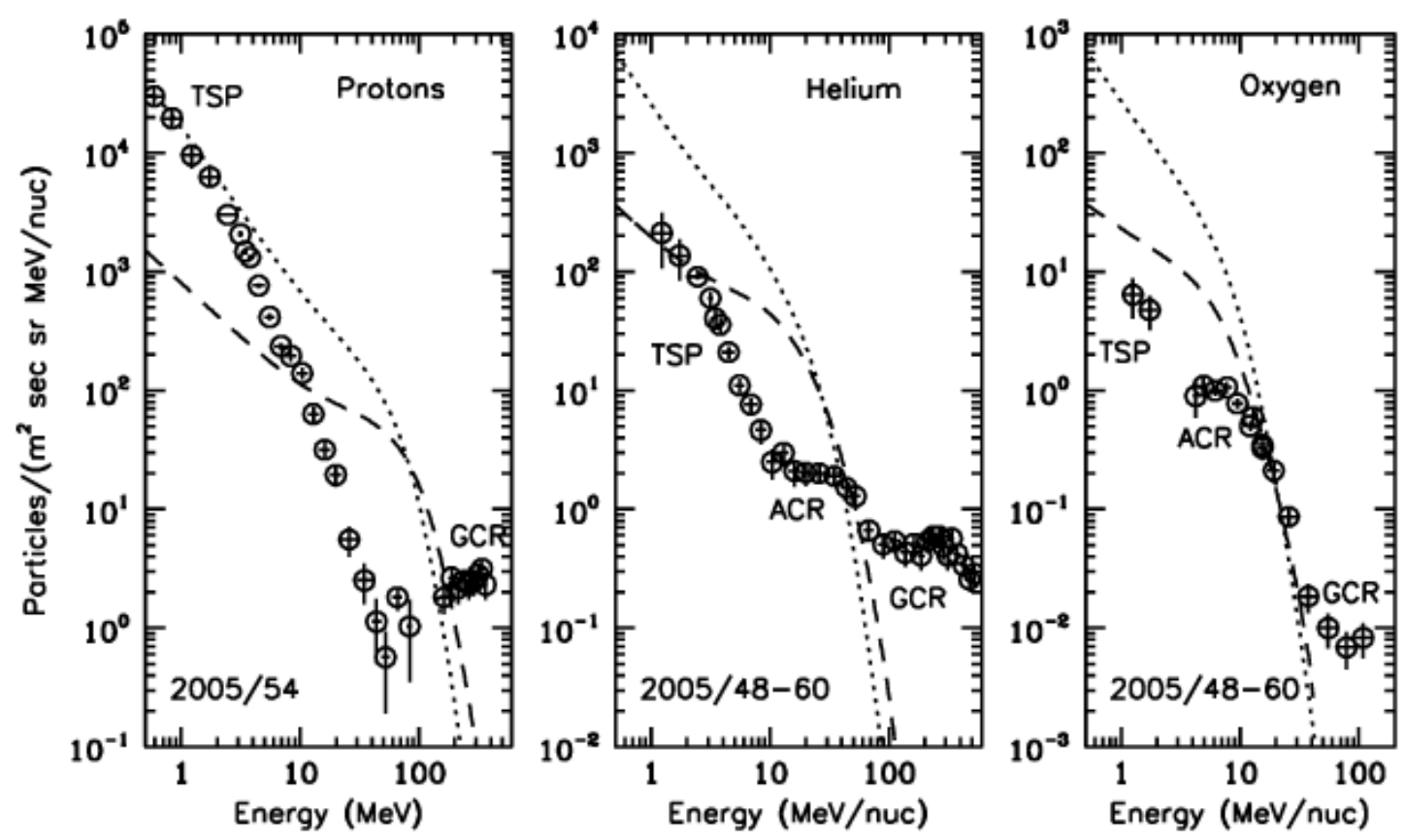

Figure 2.7.: Proton, helium and oxygen spectra for termination shock particles (TSPs), ACRs and GCRs as measured by Voyager 1. Taken from [Stone et al., 2005].

criterion for ions to be injected into the termination shock acceleration process is the presence of sufficiently strong magnetic turbulence to enable cross-field diffusion to occur [Baring, 1997]. Efficiency of the injection into this acceleration mechanism depends strongly on how oblique the shock is and also on $\eta$ where $\eta$ is defined by:

$$
\eta=\frac{\lambda_{\|}}{r_{g}}
$$

Where $\lambda_{\|}$is the parallel mean free path of the ions and $r_{g}$ is the ion's gyroradius. Thus a highly oblique shock results in a strong injection such as at the termination shock where the shock geometry is perpendicular. Without scattering or turbulence enabling cross-field diffusion, injection becomes more difficult at oblique shocks as particles which have been heated within the shock by only a small amount struggle to return to the shock in order to continue gaining energy and instead can tend to stream along the oblique field lines after only a 'partial' acceleration.

If a flow is allowed to have a compressive discontinuity in it, such as a shock, then acceleration can occur there. The acceleration happens for angles between the shock normal and the magnetic field direction. For quasi-perpendicular shocks the acceleration mechanism is more closely related to $\vec{V} \times \vec{B}$ drifts. Theories require that the scattering frequency needs to be much less than the ion's gyrofrequency so 
that the ions can remain in the shock region for extended periods of time but the scattering must also be strong enough so that the particles have a nearly isotropic distribution. Generally there is an energy threshold beyond which the spectrum drops rapidly due to particles drifting off of the shock. This cut-off is not necessarily sharp as some particles may have more or less than this energy:

$$
T_{c} \approx Z e(\delta \phi)_{\max }
$$

Where $Z e$ is the charge of the ion and $\delta \phi$ is the electrostatic potential of the shock [Jokipii and Giacalone, 1996].

Particles only gain a small amount of energy each time they cross the shock however so it can take a long time for them to gain the energy seen in anomalous cosmic rays. For quasi-perpendicular shocks this can take on the order of a year and much longer for parallel shocks or other methods of acceleration. This is, again, typically due to a first order Fermi Diffusive Shock Acceleration (DSA) mechanism and anomalous cosmic rays have energies in the region of $10-100 \mathrm{MeV}$. It should be noted that the crossing of the termination shock by the Voyager spacecraft has suggested that ACRs are not accelerated there as no evidence of a DSA mechanism was found or at least if they are accelerated at the termination shock then it is in regions away from where the spacecraft made their crossings [Stone et al., 2005]. This subject remains controversial.

\subsubsection{Shock Acceleration of Interstellar Pickup Ions}

One of the more important acceleration mechanisms of interstellar pickup ions occur at co-rotating interaction regions. A co-rotating interaction region is formed when the fast solar wind flow catches up to the slow solar wind due to its co-rotation with the Sun. Over time the fast solar wind causes compression in this interface until, just past $1 \mathrm{AU}$, it steepens into a shock with both forward and reverse shock regions. This travelling interplanetary shock has the potential to accelerate ions that it encounters [Malkov and Drury, 2001].

Lee et al. [1996], Sagdeev [1966], Zank et al. [1996] investigated the theory that pickup ions could be accelerated by 'surfing' the shock, that is, that they can encounter the shock front and become trapped between the electrostatic potential of the shock and the upstream Lorentz force. As pickup ions have the potential to be either very slow or stationary when they encounter the shock and therefore can remain in this region for a longer period of time than the solar wind ions and can 
therefore be accelerated by the motional electric field along the shock surface.

That said, however, there remain issues with the idea of acceleration by shock surfing as 2D simulations show that 'ripples' are produced which may affect the particles [Burgess, 1995] and thus DSA appears to be the favoured mechanism. This acceleration mechanism, also known as first order Fermi acceleration, occurs through slightly different paths at each shock and for pickup ions compared to solar wind protons. The mechanism itself relies on both the particle gaining some amount of energy when it crosses the shock front and on it being able to cross back and forth over the shock multiple times before it escapes from the shocked region.

If an ion approaches the shock with some velocity in the fast solar wind flow, directed towards the slow flow interface, then eventually it will cross the shock front. Since the flow it is approaching is moving more slowly than the flow in which it currently resides in the ion's frame of reference the slow solar wind will be travelling towards the ion with the speed $\left|U_{1}-U_{2}\right|$ where $U_{1}$ and $U_{2}$ are the fast and slow solar wind bulk flow speeds respectively [Baring, 1997]. Due to this relative motion once the ion has crossed the shock it has a very high chance to take part in a head-on 'collision' with a moving turbulent region or a wave. In the local fluid frame the individual particle's momentum and energy is conserved after the collision but in the frame of reference of the shock interface the particle will gain a modest amount of energy which is determined by the compression factor of the shock.

As the turbulence causes the particles crossing the shock front to isotropise in the local fluid frame and hence they then can diffuse back across the shock front after some period of time. Again, in crossing the shock the opposite way the particle once more 'sees' a flow approaching it head-on and once it has diffused across the shock front it will again be isotropised in the other fluid frame and thus will gain energy. The turbulence and/or waves will allow the particle to cross the shock and continue to gain energy in this way and hence be accelerated until the particle has gained enough energy that it can escape the shocked region. This energy gain is always positive as the collisions will always be head-on in the particle frame due to the geometry of the converging flows.

The ram energy of the solar wind can be as high as $1 \mathrm{keV}$ but the thermal energy of the solar wind protons is generally less than $10 \mathrm{eV}$ and it can therefore be difficult to accelerate such low energy particles, pre-acceleration is often a requirement before they can be injected into the DSA mechanism [Gloeckler et al., 1994]. In a quasiperpendicular DSA picture, which is standard for a CIR, a thermal solar wind proton will be swept downstream by the motion of the bulk solar wind flow due to the fact the field is frozen to it and thus such a particle cannot realistically perform multiple 
crossings of the shock boundary in order to be accelerated. For a quasi-parallel shock large amplitude waves are generated by beam instabilities which causes the solar wind protons to be pitch angle scattered and thus they are then able to diffuse across the shock front multiple times. In this case even the low energy particles can be accelerated to the observed energy of $10 \mathrm{keV}$. Typically pickup ions already have thermal speeds larger than that of the solar wind protons so that they have sufficient energy already to be injected into the acceleration mechanism and be further accelerated by the shocks.

At the forward shock of a co-rotating interaction region the pickup ions have a similar profile to the solar wind protons due to the increase in solar wind number density also increasing the frequency of ionising collisions and hence the production of pickup ions. In the reverse shock, which still has a quasi-perpendicular geometry, the energy distribution of the pickup ions displays an energetic tail. This is evidence that DSA is occurring at the reverse shock for the PUI population and that they are being accelerated there [Giacalone and Jokipii, 1997, Scholer, 1999]. Hybrid simulations of this were performed by Tsubouchi [2014] who found that PUIs could be efficiently accelerated in the vicinity of the shock surface of a corotating interaction region. Interstellar pickup ions are injected a lot more efficiently into the acceleration mechanism than are the solar wind protons. This is likely due to the process having some minimum energy requirement and the pickup ions having more energy than the solar wind protons. Additionally, it appears that hydrogen ions are injected more easily than are helium ions within a class of particles based on their source of origin (interstellar or solar). That is, picked up hydrogen is injected more easily than picked up helium and similarly for hydrogen and helium of solar origin although the solar origin species are injected less efficiently than their pickup ion counterparts.

As a result of this interstellar $\mathrm{H}^{+} / \mathrm{He}^{+}$are the most abundant suprathermal species found in CIRs. Also pickup ions of interstellar origin carry 15-20\% of the total ion plasma pressure due to these accelerated ions. This acceleration of pickup ions at co-rotating interaction regions can also be seen as the pre-acceleration phase for the generation of anomalous cosmic rays. It has also been suggested that pickup ions can also be reflected between two co-rotating interaction regions providing that they are magnetically connected, gaining energy at each reflection and can thereby also be accelerated to energies of the order of $100 \mathrm{keV}$ [Tsubouchi, 2014]. 


\subsection{Pickup Ions at Comets}

Cometary nuclei are unmagnetised and small and so therefore provide little direct impediment to the flow of the solar wind. When a comet nears the sun, however, volatiles are heated and sublime to form a neutral atmosphere/exosphere around the comet. These neutrals can be ionised by photo-ionisation or charge exchange, among other processes, at which point they are able to interact with the solar wind flow directly [Coates, 2004, Cravens and Gombosi, 2004].

Comets visited include Halley by Vega 1, Vega 2, Suisei and Giotto, GiacobiniZinner by ICE, Grigg-Skjellerup but Giotto, Borelly by Deep Space 1 and most recently Churyumov-Gerasimenko by Rosetta [Itoh and Hirao, 1998, Pätzold et al., 1997, Richter et al., 2011, Schwehm, 2006, Wenzel, 1985]. From the various in situ measurements of comets by spacecraft it can be seen that cometary environments are not all the same. Wave generation and the amplitude of those waves, among other things, often changes from comet to comet [Galeev et al., 1988, Johnstone, 1995, Laakso, 1991, Neugebauer, 1990, Verigin et al., 1987]. This can be due to many factors such as the orientation of the solar wind flow velocity or the interplanetary magnetic field with respect to the velocity vector of the comet but it is likely that the dominant factor which determines the 'strength' of the comet is its mass production rate.

The following section will discuss the aspects of a comet's interaction with the solar wind focusing on the ions which are picked up as the comet's neutral atmosphere is ionised. Topics such as general mass loading, expected velocity distributions and instabilities both observed and predicted theoretically will be covered.

As a comet's heliocentric distance decreases and its gas production rate increases a comet will develop a neutral atmosphere which will extend some distance from the cometary nucleus depending on the specific gas production rate. Whilst far from the comet the interactions are predominantly collisionless the innermost region will be collisional due to the high density. This neutral atmosphere will be ionised constantly by various processes and injection of ions onto the solar wind will act to cause mass loading. These ions are accelerated up to the solar wind velocity in the solar frame of reference at the expense of reducing the solar wind flow speed locally [Szegö et al., 2000, Terasawa et al., 1986]. As the magnetic field is frozen to the solar wind flow this will eventually result in large scale draping of the magnetic field around the comet. This draping causes a region of enhanced cometary ion number density to form which eventually becomes the comet's plasma tail which is swept along by the solar wind in contrast to the comet's dust tail which is neutral and 
is left in the wake of the comet but offset by radiation pressure. Figure 2.8 shows a schematic picture of the cometary environment far from the sun where the mass loading is weaker and close to the sun where the mass loading becomes stronger [Coates, 2004].

If the mass loading is high enough then this will cause the magnetic field lines to begin to pile up ahead of the comet and will eventually form a bow shock. Ultimately the ambient magnetic field will be completely excluded from the inner coma and a field free diamagnetic cavity will be formed. The bow shocks that form ahead of comets in this way can be of the same scale as the magnetospheres of Neptune and Uranus (see Figure 2.9 in section 2.4) depending on the gas production rate of the particular comet in question whereas the tails of comets can extend for several AU. It should be noted that the rate of addition of mass needs to pass a certain threshold before the solar wind flow becomes unable to accommodate it quickly enough and a bow shock begins to form.

In velocity space the cycloid has a ring distribution. Ring velocity distributions are unstable and waves are produced. These waves act to scatter the particles first in pitch angle so that the ring distribution becomes a thin shell and secondly in energy which thickens the shell and brings it closer to an isotropic spherical distribution. Figure 2.1 shows a schematic view of the ring and shell distributions. This shows that sufficiently high injection rates of newborn ions can perturb the solar wind flow very significantly whether this is due to a gradual build-up of ion densities or if it is due to a massive ion 'explosion' (e.g. AMPTE, an active release experiment which is discussed in more detail in section 2.5) seems not to affect the fact that a cavity is formed, just the instantaneous rates of injection.

Cometary pickup ions typically have a relatively cold distribution. Where their pitch angles are very small $\left(\alpha \approx 0^{\circ}\right)$ they form a cold beam and when the pitch angle is large $\left(\alpha \approx 90^{\circ}\right)$ they form a cold ring velocity distribution. Intermediate pitch angles form a ring-beam distribution. These types of velocity distribution are unstable to ultra-low frequency waves at, and around, the heavy ion gyrofrequency. In practice this usually corresponds to water group ions in cometary environments. These ultra-low frequency waves then interact with the newborn pickup ions via pitch angle scattering. The distribution then becomes more shell-like and that shell eventually broadens over time as the particles are also scattered in energy.

The types of waves and instabilities seen at comets initially depends on the pitch angle of the newborn ions and hence their specific velocity distribution in space and the injection geometry [Tsurutani, 2013]. That is, the angle between the magnetic field and the solar wind velocity, this is due to the fact that the pitch angle largely 
controls the initial distribution function of the pickup ions and thus the types of instability those distributions are unstable to. An extensive review of waves of all kinds observed in a cometary environment can be found written by Glassmeier et al. [1997] and Brinca [1997]. Generally the solar wind bulk flow speed is much higher than the velocity of the neutral cometary atoms or the thermal speed of the newly created ions and hence the ion speed can often be neglected.

In the perpendicular case where the pitch angle of the particles is close to $90^{\circ}$ there are three main low frequency instabilities which can arise.

1. Ion Cyclotron Instability

2. Parallel propagating non-oscillatory mode

\section{Fluid Mirror Instability}

If $\alpha$, the particle's pitch angle, is not exactly $90^{\circ}$ and there is a parallel component of the ion's velocity directed along the field such that $V_{\|} \geq V_{A}$ then the ion cyclotron instability will produce left hand waves propagating anti-parallel to the ions [Gary and Madland, 1988, Gary and Sinha, 1989, Le et al., 1989, Miller et al., 1991a,b, Otani, 1988].

The mirror mode instability is more prevalent in planetary and cometary magnetosheaths [Coates et al., 1996]. The condition to be satisfied for the fluid mirror mode is [Tsurutani, 2013]:

$$
\frac{\beta_{\perp}}{\beta_{\|}}>1+\frac{1}{\beta_{\perp}}
$$

So that this instability can only occur where either the plasma beta is high or there is a strong anisotropy. This is typically satisfied in planetary bow shocks and near large comets due to the large pickup rates generating a large anisotropy. This mode can be identified by large changes in $|B|$ but with very small directional fluctuations. This is due to the maximum growth rate of this instability being at large angles to $\vec{B}$, that is, in the region of $60-80^{\circ}$.

The mirror instability occurs in a similar parameter regime to the ion cyclotron instability. The ion cyclotron instability occurs for frequencies below the ion gyrofrequency but usually has a higher growth rate than the mirror mode and so it can reduce the anisotropy which the mirror mode requires and hence suppress it. However, it has been shown by Price et al. [1986] that the presence of heavy ions can reduce the growth rate of the ion cyclotron instability without affecting the growth rate of the mirror mode instability [Winske and Gary, 1986]. 
At very low frequencies the perpendicular pressure responds to $\vec{B}$ compression in antiphase for a bimaxwellian [Southwood and Kivelson, 1993]:

$$
\delta P_{\perp}=2 P_{\perp}\left(1-\frac{T_{\perp}}{T_{\|}}\right) \frac{\delta \vec{B}}{\vec{B}}
$$

so if $\frac{T_{\perp}}{T_{\|}}>1$ then $\delta P_{\perp}$ decreases as $\delta \vec{B}$ increases. Since $\delta P_{\perp} \propto P_{\perp}$ if $P_{\perp}$ is large enough then the sense of the pressure may change to be opposite in sense compared to the magnetic pressure. The condition for this for a bimaxwellian is:

$$
\delta P_{\perp}+\frac{\vec{B} \delta \vec{B}}{\mu_{0}}<0
$$

which results in the previously stated condition for instability. The force from the total pressure causes the plasma to accelerate and then to have a displacement from equilibrium of $\xi$ so that the equation of perpendicular motion is:

$$
\rho \gamma^{2} \xi=-i k_{\perp}\left(\delta P_{\perp}+\frac{\vec{B} \delta \vec{B}}{\mu_{0}}\right)
$$

which ultimately yields:

$$
\gamma^{2}=-k_{\perp}^{2} V_{A}^{2}\left(1+\beta_{\perp}\left(1-\frac{T_{\perp}}{T_{\|}}\right)\right)
$$

which predicts waves when $\gamma^{2}<0$. The mirror mode instability depends strongly on the spatially structured nature of the field disturbances. It grows due to a coupling between a group of particles with a small parallel velocity and the rest of the population. When the pressure anisotropy is large enough that an increase in $\vec{B}$ results in a decrease of total pressure then this causes the field lines to move closer together and hence the instability develops.

The perpendicular energy of the particles increases with a corresponding decrease in parallel energy. This is usually described by invoking a 'mirror force'. This mirror force can be thought of as acting to squeeze the plasma out of high field regions and into low field regions. Due to conservation of the magnetic moment the movement of a particle from a high to a low field region results in conversion of its perpendicular energy into parallel motion. This is contained in:

$$
\mu=\frac{m v_{\perp}^{2}}{2 B}
$$


and the conservation of energy of the particle means that its parallel velocity must increase as its perpendicular velocity decreases. Thus the mirror force can be viewed as a pseudoforce acting from regions of high magnetic field strength towards regions of low magnetic field strength.

The fluid picture of the mirror instability is not, however, completely correct as it does not produce oscillatory motion and a full treatment requires a kinetic approach to explain this [Southwood and Kivelson, 1993]. The kinetic theory states that the mirror mode is a time stationary, damped perturbation when it is stable. The reason for this, ultimately, is because not all ions respond to the perturbation in the magnetic field in the same way. Particles with a pitch angle close to $90^{\circ}$, and hence which have a small parallel velocity component, are not affected by the mirror force to the same extent as are those particles with a pitch angle far from $90^{\circ}$. The resonant particles in this case are those particles with pitch angles close to $90^{\circ}$. Those in a region where the magnetic field is decreasing will see only a continually decreasing field and likewise those in regions of increasing field will only see an increasing field all due to their lack of a significant parallel velocity component. As the instability develops the resonant particles in regions of decreasing field cool whereas those in regions of increasing field are heated and thus these particles have an energy that varies. It should be noted that the bulk of the particles are nonresonant and travel through the mirror structure seeing regions of both high field and low field and thus their energies remain roughly the same but with their velocity shifting between parallel and perpendicular components.

In the parallel case two main types of instabilities are generated:

- Right hand resonant ring beam instability

- Firehose instability

The right-hand resonant instability can have strong wave growth for $\alpha$ up to $70^{\circ}$ as it is driven by both the free energy associated with parallel motion as well as the gyromotion [Cramer et al., 1999]. The cyclotron resonance condition is:

$$
\omega=\vec{k} \cdot \vec{v}+n \Omega_{i}
$$

Where $\omega$ is the wave frequency, $\vec{k}$ is the wave vector, $\vec{v}$ is the wave velocity in the same directional sense as $\vec{k}, \mathrm{n}$ is some integer $\left(0, \pm 1, \pm 2\right.$ etc.) and $\Omega_{i}$ is the ion gyrofrequency.

The firehose instability [Coates et al., 1996] has the potential to be marginally 
stable at comets where:

$$
P_{\|}-\left(P_{\perp}+\frac{B^{2}}{\mu_{0}}\right)>0
$$

This is due to the pressure of the water group ions in the upstream region of certain comets such as Halley. Typically in cometary environments this instability does occur but is quickly quenched by the production of low frequency waves and thus is not a particularly long-lived instability. The firehose instability is possible wherever there is a flux tube of plasma. Whenever the flux tube becomes bent then there appears an outward centrifugal force which acts to bend the flux tube further whilst thermal pressure and magnetic tension act to straighten the flux tube such that we can write:

$$
\frac{m_{i} n_{0} v_{t h \|}^{2}}{R}=\frac{P_{\perp}}{R}+\frac{B_{0}^{2}}{\mu_{0} R}
$$

This instability can excite long wavelength Alfvèn waves and the flux tube can perform transverse oscillations like a firehose might.

The firehose instability grows when the parallel pressure is large compared to the perpendicular pressure and magnetic pressure. I.e. it is unstable when: $P_{\|}>$ $P_{\perp}+\frac{B^{2}}{4 \pi}$. This instability requires high beam speeds and/or relative densities. For example, the beam speed required might be as large as $15 V_{A}$ for low densities.

The right-hand resonant waves are seen by the ions as left-hand polarised. This is due to the ion beam overtaking the waves and causing an anomalous Doppler shift. The waves will propagate in the opposite direction to the solar wind flow, that is, towards the Sun. For waves propagating parallel to $\vec{B}$ their phase velocity is usually lower than the solar wind velocity so that the waves will also be Doppler shifted in the spacecraft frame where they will be seen as left-hand polarised.

The firehose instability generates low frequency left-hand polarised waves travelling anti-parallel to the ion beam. The main way to differentiate these waves from the Doppler shifted resonant waves is to look at the wave frequency. Typically firehose waves will be detected at lower frequencies than the resonant waves and also not actually at resonant frequencies.

There are two main instabilities found in cometary environments:

- Electromagnetic Ion Cyclotron (EMIC) instabilities which propagate parallel to $\vec{B}$

- Electrostatic Lower Hybrid instabilities which propagate (mostly) perpendicular to $\vec{B}$

For the EMIC instability the dispersion relation, with + corresponding to the 
regular Doppler shifted waves and the - corresponding to the anomalous Doppler shifted waves, is [Szegö et al., 2000]:

$$
D^{ \pm}\left(k_{\|}, \omega\right)=1-\frac{c^{2} k_{\|}^{2}}{\omega^{2}}+\sum_{j} \frac{\omega_{p j}^{2}}{\omega} \int d^{3} \vec{v} \frac{\nu_{\perp}}{2} \frac{1}{w \pm \Omega_{j}-k_{\|} \nu_{\|}} G(f)=0
$$

Where:

$$
G=\frac{\partial}{\partial \nu_{\perp}}+\frac{k}{\omega}\left(\nu_{\perp} \frac{\partial}{\partial \nu_{\|}}-\nu_{\|} \frac{\partial}{\partial \nu_{\perp}}\right)
$$

The ion cyclotron instability is associated with the free energy supplied by an anisotropy in the ion velocity distribution. It occurs where the perpendicular temperature is greater than the parallel temperature. The threshold of anisotropy for this instability is relatively low compared to competing instabilities such as the mirror mode and can cause strong pitch angle scattering at low levels of wave activity.

The ion cyclotron instability stimulates the growth of very low frequency, left hand polarised waves for $\vec{k} \times \vec{B}=0$ at and just below the cyclotron frequency of the ions which are driving it. For cometary environments this is typically at and near the cyclotron frequency of water group ions. For hotter ion velocity distributions the growth rate of the instability decreases and so too does the real frequency of the waves produced. Hence the waves observed will typically be at and just below the cyclotron frequency and can be significantly below the cyclotron frequency for hotter distributions.

For comets with low mass production rates, such as Grigg-Skjellerup, the wave production starts much closer to the cometary nucleus than for larger comets like Halley and so there is less pitch angle scattering for the resonant ions and the velocity distribution will remain closer to a ring than a shell. Additionally due to the lower injection rate at smaller comets the velocity distribution can also be non-gyrotropic since the ring distribution is not filled homogeneously over a gyroperiod. This also depends on the average pickup time of the newborn ions.

For ion cyclotron waves to be stimulated in a plasma it requires that the magnetic fluctuations are in resonance with the particle motion. The fields, as seen in the frame of reference of the particle must remain stationary for a significant length of time. The resonance condition is:

$$
\omega-\vec{k} \cdot \vec{v}= \pm n \Omega_{i c}
$$

Where $\omega$ is the wave frequency, $\vec{k}$ is the wavenumber, $\vec{v}$ is the velocity of the ion, 
$\mathrm{n}$ is the harmonic number of the wave and $\Omega_{i c}$ is the cyclotron frequency of the ion species [Gary, 1991].

The lower hybrid [Brinca and Tsurutani, 1988] wave frequency for slightly oblique waves is:

$$
\begin{aligned}
\omega & =\left(\frac{\omega_{p}^{2}+\omega_{e}^{2} \frac{k_{\|}}{k^{2}}}{\epsilon_{\perp e}}\right)^{\frac{1}{2}} \\
& =\left(\frac{\Omega_{e}^{2}\left(\omega_{p}^{2}+\omega_{e}^{2} \frac{k_{\|}^{2}}{k^{2}}\right)}{\omega_{e}^{2}+\Omega_{e}^{2}}\right)^{\frac{1}{2}}
\end{aligned}
$$

Where $\epsilon$ is the dielectric constant:

$$
\epsilon_{\perp e}=1+\frac{\omega_{e}^{2}}{\Omega_{e}^{2}}
$$

Left hand waves always have a frequency between the electron and proton gyrofrequencies. Ions in the left hand waves do not feel the $\vec{B}$ field but the electrons are strongly magnetised and experience Landau resonance: $\omega=k_{\|} \nu_{\|}$but only along the field lines. As $k_{\|}<<k$ the left hand waves can simultaneously be in resonance with both electrons and ions and can thus transfer energy from slow ions to fast electrons.

After comparing the growth rates of the ion cyclotron and left hand instabilities it can be shown that well upstream of cometary bow shocks the ion cyclotron instability is dominant and the left hand growth rate does not become significant until within the bow shock [Szegö et al., 2000]. Drifting proton rings generate stronger parallel wave growth than the heavier water group ions. If wave activity observed by a spacecraft originated in resonant instabilities then for general propagation directions it will satisfy:

$$
\omega-\vec{k} \vec{v}_{b \|}=n \Omega_{b}
$$

i.e. it will be at, or very close to, cyclotron resonance frequencies.

Pitch angle scattering is not as effective at smaller comets such as GiacobiniZinner and Grigg-Skjellerup [Shevchenko et al., 1995] and the ions largely retain their ring-beam velocity distribution. This contrasts to the larger comets like Halley where ions are nearly isotropic within $\approx 10^{6} \mathrm{~km}$ of the bow shock [Le et al., 1989]. One advantage of the smaller comets are that they retain the ring-beam structure and it is easier to see the waves excited by the water group ions at $\omega_{s c} \approx-\Omega_{O H}$ 
with left hand circular polarisation of the waves whereas at the larger comets with more effective pitch angle scattering this would be seen more as an enhancement to the background turbulence near those frequencies. Newborn cometary ions with a ring velocity distribution can excite lower hybrid waves via a loss cone instability: the modified two stream instability (MTSI) [Shapiro et al., 1999]. This requires a minimum gas production rate though and so only the larger comets can see this lower hybrid activity.

The Kelvin-Helmholtz instability happens where layered flows of immiscible, incompressible and inviscid fluids can be found with a velocity shear between the layers. The shear flow induces vorticity at the fluid boundary so that a vortex sheet is formed which then rolls up. Comet Churyumov-Gerasimenko may have a region at the edges of its diamagnetic cavity which, due to the asymmetric production of neutrals and hence ions, is unstable to the Kelvin-Helmholtz instability [Rubin et al., 2011]. Due to mass loading in the local region of the comet there will be regions of shear flow produced as the less mass loaded solar wind is forced around the edge of the cavity and the slower, more mass loaded solar wind. This shear flow can cause Kelvin-Helmholtz instabilities to develop. It has been predicted by Rubin et al. [2011] that such cavities are more likely to be stable at small heliocentric distances but as the comets heliocentric distance increases it can become unstable due to the reduction of the local magnetic field strength despite the mass loading also decreasing and that signatures of the Kelvin-Helmholtz instability might be seen in the plane perpendicular to the ambient magnetic field.

Observations of waves near to comets are often of low frequency hydromagnetic waves at or near to the water group gyrofrequency [Yumoto et al., 1987]. Wave activity in general is seen to drop off with increasing distance from the comet and a higher level of wave activity is observed within the cometosheath [Grard et al., 1987]. Mirror mode waves have also been observed at the larger comets, such as Halley, where there is a sufficiently large pressure anisotropy as well as fast magnetoacoustic modes [Glassmeier et al., 1993]. In addition to this, observations have also been made of intense lower hybrid wave activity near Halley [Shapiro et al., 1993]. Evidence of proton cyclotron waves was also seen by Tan et al. [1993] by computing frequency spectra of power density and polarisation parameters of the MHD waves seen near Giacobini-Zinner by ICE during a fly-by. Most recently Richter et al. [2015] have reported observations of a new type of quasi-coherent, large amplitude, compressional, low-frequency wave at Churyumov-Gerasimenko which they claim is a result of a cross-field current instability associated with the newborn cometary ions. 


\subsection{Pickup Ions at Planets and Moons}

The following section will review the interactions of pickup ions in various situations which are, in some way, unique to the planetary environments. These include such situations as the heavy ion torus around Jupiter, Venus with its dense atmosphere and induced magnetic field or Mercury with an intrinsic magnetic field and tenuous atmosphere. When pickup ions are born they often have non-equilibrium velocity distributions which can excite short wavelength normal modes of the plasma; microinstabilities. These waves act to scatter the particles into a more stable velocity distribution. The properties of the waves excited by the various instabilities can be used to infer things about the pickup ion distribution that stimulated them and hence also about the neutral population that they came from. Planets without a magnetic field will be continuously losing particles from their atmosphere due to the interactions with the solar wind.

In the solar system there are various planetary pickup configurations available to study. Mercury has a global magnetic field which is very weak, only about $1 \%$ of the strength of Earth's magnetic field, and it also has a very thin atmosphere which means that capture by its magnetosphere of species in the solar wind is fairly low and that the tenuous atmosphere it does have is not at all well protected from being swept away by the solar wind interactions.

In contrast Venus has a very dense atmosphere and no intrinsic magnetic field, only a very small one induced by its direct interaction with the magnetised solar wind which provides it with negligible protection. Thus ions in the Venusian atmosphere are constantly being ionised and swept away by the accelerating effect of the solar wind.

Earth has an atmosphere which is dense in comparison to that of Mercury but it also has a reasonably strong magnetic field of its own so that it has a fully formed magnetosphere which protects the atmosphere from direct interaction with the solar wind. This means that any diffusion of solar wind ions into the magnetosphere will happen at the bow shock or in the magneto-tail and is a relatively slow process.

Saturn and Jupiter both have extensive atmospheres and magnetic fields strong enough to form their own magnetospheres. Jupiter, having a strong intrinsic field, has the larger magnetosphere. The main differences these planets have with respect to Earth is that each has a satellite which is ejecting significant amounts of mass into the magnetosphere of its parent planet. Jupiter has Io ejecting sulphur molecules and Saturn has Enceladus ejecting water group molecules. This results in unique features not seen elsewhere. Additionally within the Kronian magnetosphere it has 
been proposed that Titan, which is the only natural satellite known to have a dense atmosphere, is a source of nitrogen pickup ions.

\subsubsection{Jupiter}

Io is volcanically active and adds in the region of $1 \mathrm{kgs}^{-1}$ of material to the local region of space. This is mostly comprised of sulphuric ions with $\mathrm{SO}^{+}$and $\mathrm{SO}_{2}^{+}$being the dominant species.

The Jovian magnetic field is roughly perpendicular to the plane of the resultant plasma torus so the newborn ions will form a ring distribution in velocity space, similar to the behaviour of ions at comets, in the frame of the corotating torus. As previously mentioned, these distributions are unstable to ion cyclotron instabilities [Blanco-Cano et al., 2001, Cowee et al., 2008, Dols et al., 2012]. At Io whilst the newly born pickup ions have a ring beam distribution (more or less) ion cyclotron waves are not commonly observed there at the $\mathrm{S}^{+}$and $\mathrm{O}^{+}$cyclotron frequencies. This is due to the presence of the thermalised Maxwellian torus being present which results in the damping of ion cyclotron waves. Ion cyclotron waves at the $\mathrm{SO}_{2}^{+}$ gyrofrequency are observed as the torus is predominantly $\mathrm{S}^{+}$and $\mathrm{O}^{+}$and thus the $\mathrm{SO}_{2}^{+}$frequency mode is not as strongly damped and is observed between 7-20 $R_{I o}$ as a left handed circularly polarised wave travelling parallel to the magnetic field. This is because $\mathrm{SO}_{2}^{+}$decays too rapidly to constitute a significant fraction of the plasma torus but does not decay so rapidly that it cannot stimulate ion cyclotron waves. Thus they are not damped by the thermalised component of $\mathrm{SO}_{2}^{+}$in the plasma torus as quickly as the $\mathrm{S}^{+}, \mathrm{O}^{+}$and $\mathrm{SO}^{+}$. However, the $\mathrm{SO}_{2}^{+}$waves are damped eventually as the $\mathrm{S}^{+}$ions are non-resonantly scattered by them whereas the $\mathrm{O}^{+}$ions are not.

The Io plasma torus has a large magnetic field and a low plasma beta so observations of a mirror mode there were unexpected as it generally requires a large plasma beta and a high ratio of perpendicular pressure to parallel pressure [Russell et al., 1998]. The flank/wake of Io has a much higher beta and also a large pressure anisotropy within the current sheet where the density is highest. The mirror mode is, however, stabilised within the torus due to the quasi-isotropic distribution there. This configuration allows the mirror mode to occur and to dominate over the ion cyclotron instability in regions where the ion cyclotron instability remains damped by the ions in the plasma torus. One possible source for the anisotropy which allows the growth of the mirror mode is the re-ionisation of energetic neutral atoms (ENAs) which are produced in the plasma torus. This would provide an additional weak 
pickup source which could drive waves and instabilities [Huddleston et al., 2000].

\subsubsection{Mars}

Without a significant magnetic field to protect it from the solar wind flow, the tenuous atmosphere of Mars sits exposed directly to the effects of the solar wind. Ion cyclotron waves were detected at Mars initially by the Phobos mission at about 2-3 Mars radii near the local proton gyrofrequency [Cowee et al., 2012, Wei and Russell, 2006]. These waves are associated with the ionisation of monatomic hydrogen from the Martian exosphere. This was supported by the observations made by Phobos of ring-beam pickup ion distributions. The cyclotron waves were found to be distributed asymmetrically in the direction of the interplanetary electric field by the Mars Global Surveyor (MGS) [Fang et al., 2008, Nagy et al., 2004]. Due to the fact that the wave growth is driven by exospheric pickup this shows that the Martian exosphere is also extensive and asymmetric in the electric field direction.

Ion cyclotron waves have also been observed at large distances from the Martian exosphere and, in a similar fashion to the other planetary plasma environments, this is due to charge exchange effects. A fast ion undergoes a charge exchange event with a slow neutral which results in a slow ion and a fast neutral. The neutral can then travel unaffected by the ambient magnetic field until it is re-ionised at a larger distance and can then stimulate the growth of ion cyclotron waves far from the source of the original ions. Ions thus produced can have up to four times the solar wind flow speed.

Phobos 2 detected evidence of proton cyclotron waves upstream of Mars [Wei et al., 2011]. These are thought to be caused by the pickup of ions from the atmosphere. The atmosphere of Mars is eroded primarily by charge exchange and photoionisation effects. The newborn ions are picked up, gyrate around the magnetic field lines and form ring and ring-beam distributions in velocity space depending upon the angle between the solar wind flow and the interplanetary magnetic field. Such distributions are typically unstable to ion cyclotron instabilities.

Mars has a hot oxygen corona [Cravens et al., 2002], the energies of the oxygen pickup ions found there means that they must have been created several Mars radii upstream due to the fact that they have undergone acceleration. Due to somewhere of the order of half of the neutral oxygen atoms having velocities in excess of the required escape velocity, this seems feasible.

The objective of the MAVEN (Mars Atmosphere and Volatile EvolutioN) mission is to study the Martian atmosphere in order to determine how the Martian atmo- 
sphere was lost. From this study many new results will be returned and should apply especially to pickup processes around Mars which seem likely to have had an effect upon the evolution of the Martian atmosphere.

\subsubsection{Saturn}

$\mathrm{H}_{2} \mathrm{O}^{+}$ions are the dominant species near Enceladus having approximately ten times the number density of the next most abundant ion, $\mathrm{O}_{2}^{+}$[Tokar et al., 2008]. Various other water group ions are also present but due to some ambient levels of solar wind ions the pickup versions may not be detectable. Ion cyclotron waves can be seen near Dione at the water group ion cyclotron frequency [Sittler et al., 2004].

Saturn's E-ring is composed primarily of micron sized grains of water-ice and is surrounded by an exosphere of water group ions [Cowee et al., 2008]. It extends from 3-8 Saturn radii and encompasses the orbits of both Enceladus and Dione among others which are thought to be possible sources for the neutrals in this ring. A peak in the number density of neutrals at the orbit of Enceladus indicates that this is likely. Pickup ions are estimated to form approximately $8 \%$ of the total ion density within the Enceladus torus.

Titan has an extensive ionosphere which is composed primarily of nitrogen and carbon based compounds. Whilst Titan is typically within Saturn's magnetosphere it has a chance to exit the magnetosphere and interact directly with the solar wind if conditions are extreme. Titan's atmosphere also suffers losses due to sputtering effects from pickup ions [Ledvina et al., 2004]. This causes the production of a distribution of neutrals which typically have low energies but also an energetic tail. Like at Enceladus [Leisner et al., 2006], ion cyclotron waves have been detected at Titan [Russell et al., 2014]. It was thought that they would be undetectable due to the length of their growth time being large compared to the convection time of the plasma through the interaction region. Any waves produced were thought not be of sufficient amplitude to be detected as they would be swept out of the region with suitable conditions before they could grow large enough.

\subsubsection{Mercury}

Mercury has only a tenuous atmosphere but a modest intrinsic magnetic field which has sufficient strength to cause the solar wind to stand off the planet [Baumjohann et al., 2006]. A difference between the Hermean magnetosphere and the terrestrial one is that, due to the relative small size of the magnetosphere at Mercury, the 
region where charged particle populations might be expected to be trapped is so small that any such populations would be transient. A consequence of this is that where usually the planetary atmosphere is an important source of ions, at Mercury the magnetosphere couples directly to the planet's surface which causes the surface to act as both a sink for ions and also a source as ions are sputtered away [Koehn et al., 2002]. These pickup ions, especially the sodium ions, tend to have gyro-radii within an order of the size of the Hermean magnetosphere and therefore kinetic effects become important [Gingell et al., 2015, Trávníček et al., 2010].

Mariner 10 was unable to detect any $\mathrm{Na}^{+}$ion cyclotron waves at Mercury being produced by the thermalisation of the newly ionised sodium ions [Boardsen and Slavin, 2007]. Several explanations were put forth for this observed lack. First was simply that Mariner 10 traversed Mercury's magnetosphere too quickly and therefore was not present for sufficient oscillations and could thus not resolve the waves. Second was that the large gyroradius of the $\mathrm{Na}^{+}$ions caused them to exit the system quickly, before they had sufficient time to excite the ion cyclotron waves to detectable levels [Sarantos et al., 2009]. Additionally it may be that ion cyclotron waves in that environment would have a wavelength of comparable size to the system and thus such long wavelength waves may not be able to grow large enough to be able to thermalise the planetary pickup ions.

Some data returned by MESSENGER, however, suggests that there is ultra low frequency $(0.4-5 \mathrm{~Hz})$ wave activity near Mercury which may be dominantly left hand polarised [Boardsen et al., 2012]. This is indicative of ion cyclotron waves. There is also evidence for shorter wavelength waves which have been taken to be due to electromagnetic ion Bernstein modes. These waves drop off in power towards the poles which suggests an equatorial source of power.

\subsubsection{Earth}

The Moon lost all but a trace of its atmosphere early in its history and thus it is now left with only a very tenuous one. Based upon measurements of the sodium cloud which was unambiguously identified it was determined that the solar wind could not supply sufficient sodium to account for this and thus the lunar regolith must constitute a significant source for atoms in the atmosphere. Thus if the lunar surface contributes to the lunar atmosphere then once atoms in the atmosphere become ionised it can also contribute to pickup ion densities [Sadovski and Skalsky, 2014]. Indeed, several missions including AMPTE and WIND have reported fluctuations of singly charged lunar pickup ions. In the frame of reference of the moon any newborn 
ion will experience a motional electric field which is several orders of magnitude stronger than the gravitational field and this, along with the gyrophase of the ion, will determine whether the ion impacts the lunar surface once more or is swept away on its cycloid trajectory [Birch and Chapman, 2001]. Mall et al. [1998], through considerations of the local space conditions and the trajectories of the spacecraft, have determined that the fluxes of interstellar $\mathrm{O}^{+}$were not high enough and that there is likely to be a lunar source of these ions. Additionally they suggest that these are $\mathrm{Al}^{+}$and $\mathrm{Si}^{+}$ions and even with some occurrence of $\mathrm{Mg}^{+}, \mathrm{P}^{+}, \mathrm{Na}^{+}$and $\mathrm{Ar}^{+}$but that they lack the statistics to positively identify them at this time.

\subsubsection{Venus}

Venus is effectively a non-magnetic planet and to a first approximation it behaves like a highly conducting spheroid with respect to the solar wind flow. The atmosphere of Venus extends into the shocked solar wind. As Venus does not have its own intrinsic magnetic field its atmosphere stands directly exposed to the solar wind flow. Thus the magnetic tail and ionosheath will have ion components which originate from the neutral atmosphere. These ions will be created predominantly by charge exchange processes with solar wind ions and photoionisation [Luhmann, 1986].

As the neutral atmosphere is ionised and an ionosphere is formed the solar wind sweeping past cannot penetrate it and thus a wake is formed behind the planet which contains an induced magnetotail, a bow shock and magnetic barrier ahead of the planet [Curry et al., 2015]. Venus also has a hot oxygen corona which extends beyond the ionopause.

The region near the ionopause displays evidence of waves and instabilities on the dayside of the planet as well as on the nightside. There is evidence for right hand polarised waves in the tail of Venus at roughly the $\mathrm{O}^{+}$gyrofrequency [Grebowsky et al., 2004]. The energy distribution of these ions is very narrow which supports the interpretation that these ions in the tail are of ionospheric origin rather than hotter solar wind pickup ions.

It has been suggested by some authors that the number of planetary pickup ions produced affects the stand-off distance of the bow shock [Lu et al., 2013]. As more ions are produced the bow shock responds by moving further away from the planet. As the solar wind picks up the planetary ions more effectively when the velocity and the interplanetary magnetic field are perpendicular, their effect on the bow shock will be greatest at this time also. Thus pickup by the motional electric field also introduces an asymmetry in the field draped around the magnetosheath as ions are 
lost to atmospheric absorption in only one hemisphere [Phillips et al., 1987].

The influence of planetary pickup ions on the bow shock position is also a variable of solar activity. Near solar maximum the ionosphere of Venus is relatively dense and mass loading effects are the greatest whereas towards solar minimum the exosphere of Venus is tenuous enough that the planetary pickup ions have an almost negligible effect on the bow shock.

It has been shown by Luhmann et al. [2006] that the magnetosheath of Venus is disturbed on streamlines of plasma which intersect the quasi parallel section of the bow shock. These disturbances are convected into the dayside magnetosheath where ion acceleration can occur efficiently via the electric fields associated with the low frequency convected transverse magnetic fluctuations.

This pickup mechanism differs slightly from the large scale $\vec{E} \times \vec{B}$ pickup in that the $\vec{E}$ field varies in this case so that some spread in ion trajectories occurs and so there is no large scale organisation as there is for $\vec{E} \times \vec{B}$ pickup.

Theoretically pickup could occur in the same manner as at comets with the associated waves and instabilities, however, evidence for such is not obvious near Venus although there does exist evidence for both ion acoustic and whistler waves. The space environment of Venus is also similar to that of comets as in both cases the magnetic field lines being convected by the solar wind become caught on the obstacle, either the 'contact surface' of the comet, its outer cometosheath, or the ionosphere of Venus. The end result being that the IMF becomes draped around the obstacle.

It was found by Russell et al. [2006] by a reanalysis of Pioneer data using newly developed analysis techniques not available at that time that proton cyclotron waves are present in only the magnetosheath of Venus which contrasts to other situations of pickup ion driven waves where they can also be detected for significant distances (10s of planetary radii) upstream [Russell and Blanco-Cano, 2007]. It has been thought that the lack of proton cyclotron waves upstream of Venus could be considered as evidence that the solar wind does not strongly couple to the Venusian atmosphere.

\subsection{Active Release Experiments}

Active release experiments involve the controlled release of, usually large, quantities of neutral particles into the solar wind, ionosphere or magnetosphere. These neutral particles are then ionised over some time frame. For some experiments this time frame is chosen to be small (i.e. the AMPTE releases) so that the mass loading of the solar wind will be quite large. These experiments act as active probes into 
pickup processes as the initial conditions can be controlled and known to a higher accuracy than for other, naturally occurring pickup processes. The following section will review the findings of three active release experiments: Combined Release and Radiation Effects Satellite (CRRES), Active Magnetospheric Particle Tracer Explorers (AMPTE) and the Critical Ionisation Velocity Experiment (CRIT-II).

\subsubsection{CRRES}

The CRRES mission started in July 1990 and finished on October 21st 1991 and performed a series of plasma injection experiments over this duration. The intention was to investigate the interaction between the released material and the ionosphere [Blagoveshchenskaya et al., 1998]. Additionally CRRES performed several experiments to investigate critical ionisation velocity (CIV) effects, discussed later. These involved releases perpendicular to the ambient field at $9.6 \mathrm{kms}^{-1}$. Whilst this geometry was ideal for CIV processes to occur and be observed no definitive evidence was found.

The CRRES releases were performed in full sunlight with barium. In these conditions the barium cloud steadily photoionised with a time constant of 23 seconds. This time constant of photoionisation means that a smooth and uniform ion cloud would be expected to trail the neutral cloud, however, a portion of the ions were observed to move in the $\vec{E} \times \vec{B}$ direction briefly. Hybrid simulations of these releases performed by Delamere et al. [2001] gives some insight into the processes at work. Initially a polarisation electric field was set up primarily in the direction perpendicular to both the injection direction and the magnetic field. This causes the $\vec{E} \times \vec{B}$ drift to be in the original injection direction. Alfvén waves were also observed to propagate along the magnetic field lines. Ambient plasma was accelerated along the direction of injection by the Alfvén waves, thus transferring momentum from the injected barium to the ambient plasma. The ion core formed two plasmoids along the magnetic field and, due to ion gyromotion, shows an asymmetry in the plane perpendicular to the magnetic field.

Initially the entire ion cloud becomes polarised which sets up an electric field directed in the initial direction of injection but as the neutral cloud expands, ions at the edge of the cloud are able to become magnetized and will then form an ion tail. Ions in the core, however, continue to propagate.

A 'herring bone' structure is also formed by the ion cloud as the core of the ion cloud has a density which reaches a maximum for ions with a pitch angle of $90^{\circ}$ and which drops off away from that angle. Thus the ions with a pitch angle of $90^{\circ}$ will 
slow down the fastest with this effect, again, dropping off away from $90^{\circ}$. In this way the ion tail forms the observed herring bone structure.

Further, the ion cloud becomes elongated along the magnetic field which indicates that the cloud was accelerated at early times. It is suggested that this is due to ambipolar electric fields being set up by sharp density gradients at the edge of the ion cloud.

\subsubsection{AMPTE}

As part of the AMPTE (Active Magnetospheric Particle Tracer Explorers) release experiments clouds of lithium and barium were released in the upstream solar wind [Bernhardt et al., 1987, Bryant, 1985]. As the ion cloud expands the interplanetary magnetic field is pushed out of the region the cloud is expanding into. As the cloud gets larger the field lines become bent and the field is compressed at the upstream side of the cloud. As the expansion of the cloud continues the region in which the interplanetary magnetic field has been excluded grows in size and depth which eventually forms a diamagnetic cavity which extends a small distance downstream [Paschmann et al., 1986]. In addition to the IMF being pushed out of the cloud, so too is the ambient solar wind plasma. This forms a small density pulse which propagates through the solar wind just in front of the ion cloud's expansion.

The active element of the AMPTE mission constituted injections of lithium and barium into the solar wind. The injections ranged from $0.2 \mathrm{~kg}$ of lithium to $3 \mathrm{~kg}$ of barium along with small amounts of copper oxide reactant. The scientific goals were to both attempt to trace known ions (as lithium ions are scarce) and to study the interactions of different plasmas in space: the ion cloud was far denser than the ambient solar wind plasma.

The AMPTE releases demonstrate a class of interactions of a dilute, magnetized, supersonic plasma with a dense, unmagnetized, stagnant cloud. The main features of the AMPTE release are the diamagnetic cavity formed and the subsequent return of the magnetic field. The return of the magnetic field is described by a "snow plough' model. The return of the magnetic field was coincident with an increase in plasma density by a factor of five.

In December of 1984 a barium release was performed as a part of the AMPTE active release experiments [Gurnett et al., 1986, Gurnett, 1985]. The ion cloud generated from this release displayed an unexpected lateral motion of several cloud radii in the $\vec{U}_{0} \times \vec{B}$ direction rather than being accelerated downwind as was expected where $\vec{U}_{0}$ is the cloud's initial velocity vector. 


$$
\frac{\partial \vec{B}}{\partial t}=-\nabla \cdot\left(U_{0} B\right)+\frac{\alpha}{2} \hat{z} \cdot \nabla\left(\frac{1}{n}\right) \times \nabla B^{2}
$$

The explanation of the drift given by Harold and Hassam [1991] is as follows. With the field uniform, a density gradient pointing out of the cloud the second term on the right hand side of equation 2.33 is initially zero due to the uniform magnetic field. The first term, however, will tend to predict an increase in $\vec{B}$ ahead of the ion cloud and a decrease behind it which corresponds to the pileup of the magnetic field. As the field piles up the second term's significance begins to increase and the gradient in $\vec{B}$ will point upwind. With the inverted density gradient pointing out of the cloud this will cause an asymmetry in the levels of $\vec{B}$. It will increase on the right hand side and decrease on the left hand side of the cloud. Finally the cloud is accelerated due to this asymmetric gradient in $\vec{B}$. Descriptions of this drift were also given by Delamere et al. [1999], Haerendel et al. [1986], Papadopoulos and Lui [1986]

Following the second lithium release, wave activity was observed by IRM (ion release module) [Häusler et al., 1986, Roeder et al., 1987]. This wave activity appeared as a diffuse noise at approximately $80 \%$ of the electron cyclotron frequency and were polarized perpendicular to the magnetic field. Based on the data available for the particles the only realistic source of free energy to drive these waves was the drift velocity of the lithium ions relative to the solar wind ions as they are picked up. The sensitivity of the instruments meant that the determination of whether the waves were electromagnetic or electrostatic in nature could not be made although the data available suggested electrostatic.

Observations made of the waves were inconsistent with those expected if the waves were produced via a beam instability. The suggested source for these waves was a population of electrons which were stationary with respect to the spacecraft however, a definitive mechanism was never positively identified.

The lack of observations of such waves after the first lithium release is attributed to the difference in the angle between the solar wind flow direction and the interplanetary magnetic field. Additionally, there are various studies on other aspects of this release such as high energy Li ions [Möbius et al., 1986] or that the release may have caused geomagnetic pulsations [Lühr et al., 1990]. Further, general overviews are given by Jones and Rycroft [1984] and simulations were performed by Kazeminezhad et al. [1993]. 


\subsubsection{CRIT II}

CRIT II was an ionospheric injection experiment with the intent of studying the critical ionisation velocity (CIV) effect first proposed by Alfvén [Alfvén, 1954]. Shaped barium charges were detonated to create a stream of fast moving barium vapour. The twin explosions created streams of barium travelling at approximately 13.5 $\mathrm{kms}^{-1}$ [Bolin et al., 1996]. The original proposal put forth by Alfvén for the CIV effect was that a neutral gas moving through a magnetized plasma could undergo extremely rapid ionisation if the relative velocities between them perpendicular to $\vec{B}$ satisfy:

$$
v \geq v_{c}=\left(\frac{2 e U_{i}}{m_{n}}\right)^{\frac{1}{2}}
$$

Where $v_{c}$ is the critical ionisation velocity, $m_{n}$ is the mass of the neutral atom and $U_{i}$ is the ionisation potential of the atom.

The geometry of the CRIT II releases were chosen in order to maximise the opportunity for the injected barium to undergo CIV processes. It is unclear exactly how much the CIV processes contributed to the ionisation of the released barium but estimates by Liou et al. [1996] showed that $90 \%$ of the barium ions produced by the first release and $45 \%$ of the barium ions produced by the second release were due to CIV effects although there is a significant uncertainty associated with those estimates.

The most prominent low frequency feature seen in the data was an oscillation at a frequency of $5 \mathrm{~Hz}$ which is very close to the barium gyrofrequency in these conditions of $4.8 \mathrm{~Hz}$. The instability seen to occur was found to have some of the characteristics which would be expected of CIV, that is, it displayed a production of a current along the wavefront and use of the ion energy as a driving source. With this said, however, the low frequency fluctuations show that it has some differences from the properties of CIV based on theory and simulations. It should be noted that the CIV effects are still somewhat controversial and the exact ionisation mechanism involved, if it exists, is still not fully understood. 


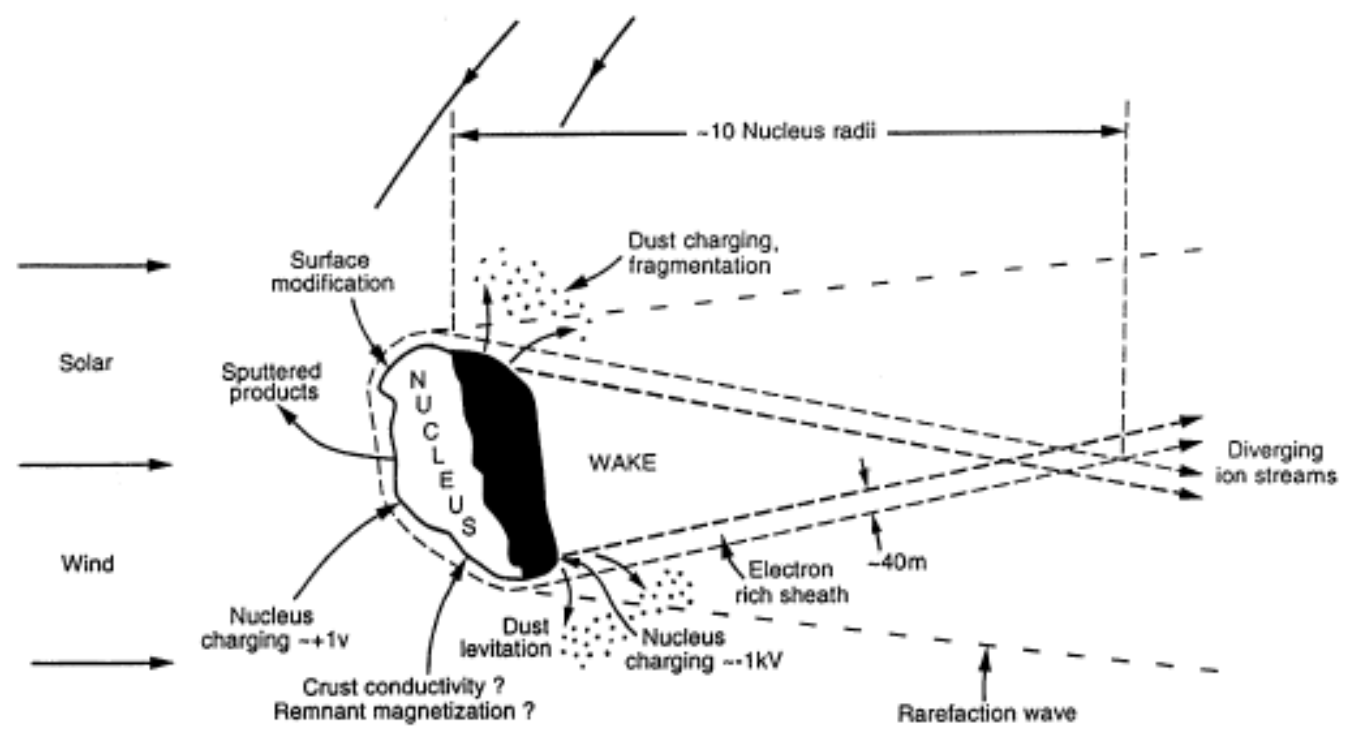

(a)
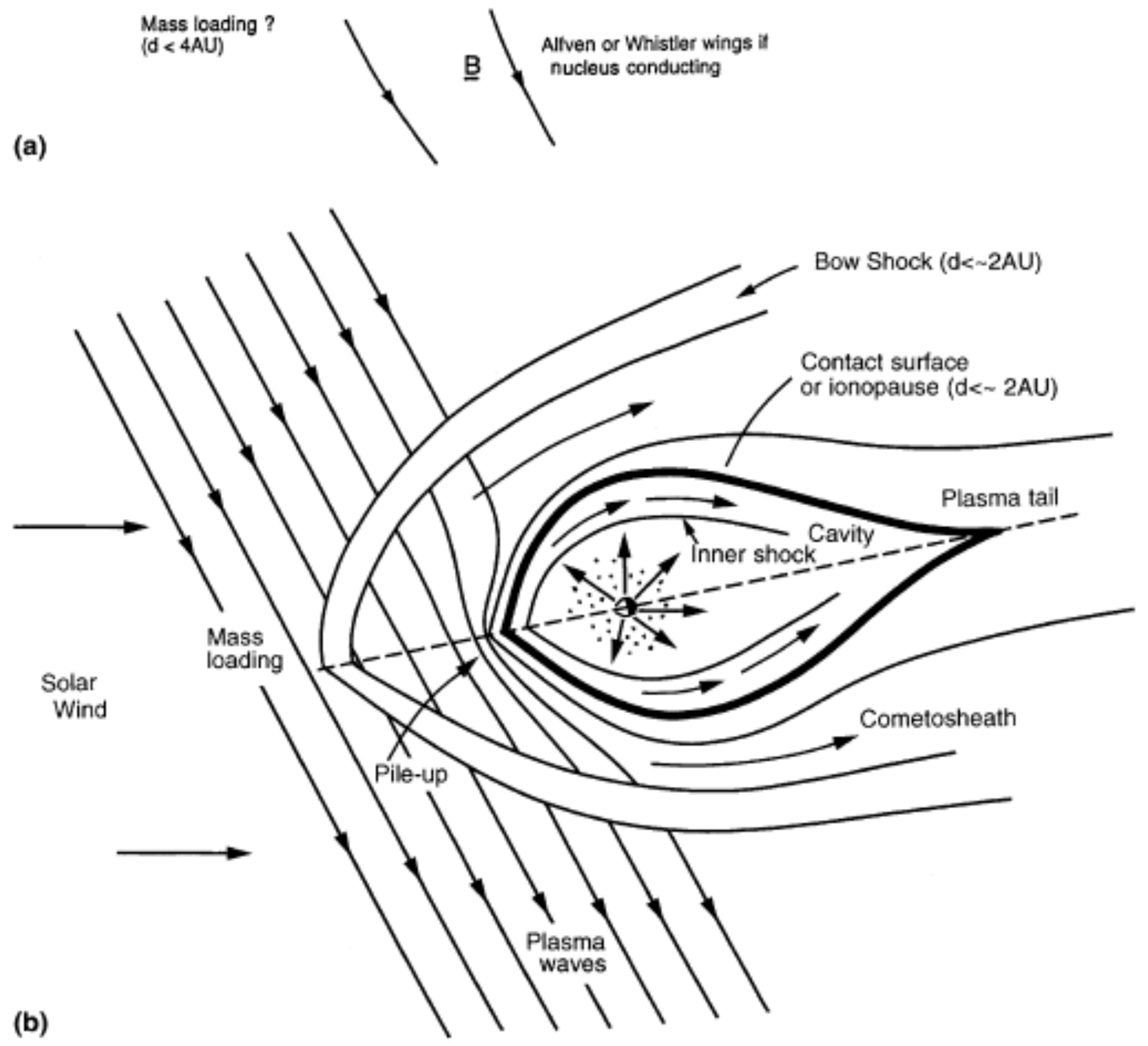

Figure 2.8.: Schematic view of the cometary environment far from the sun (a) and close to the sun (b). Taken from [Coates, 2004]. 


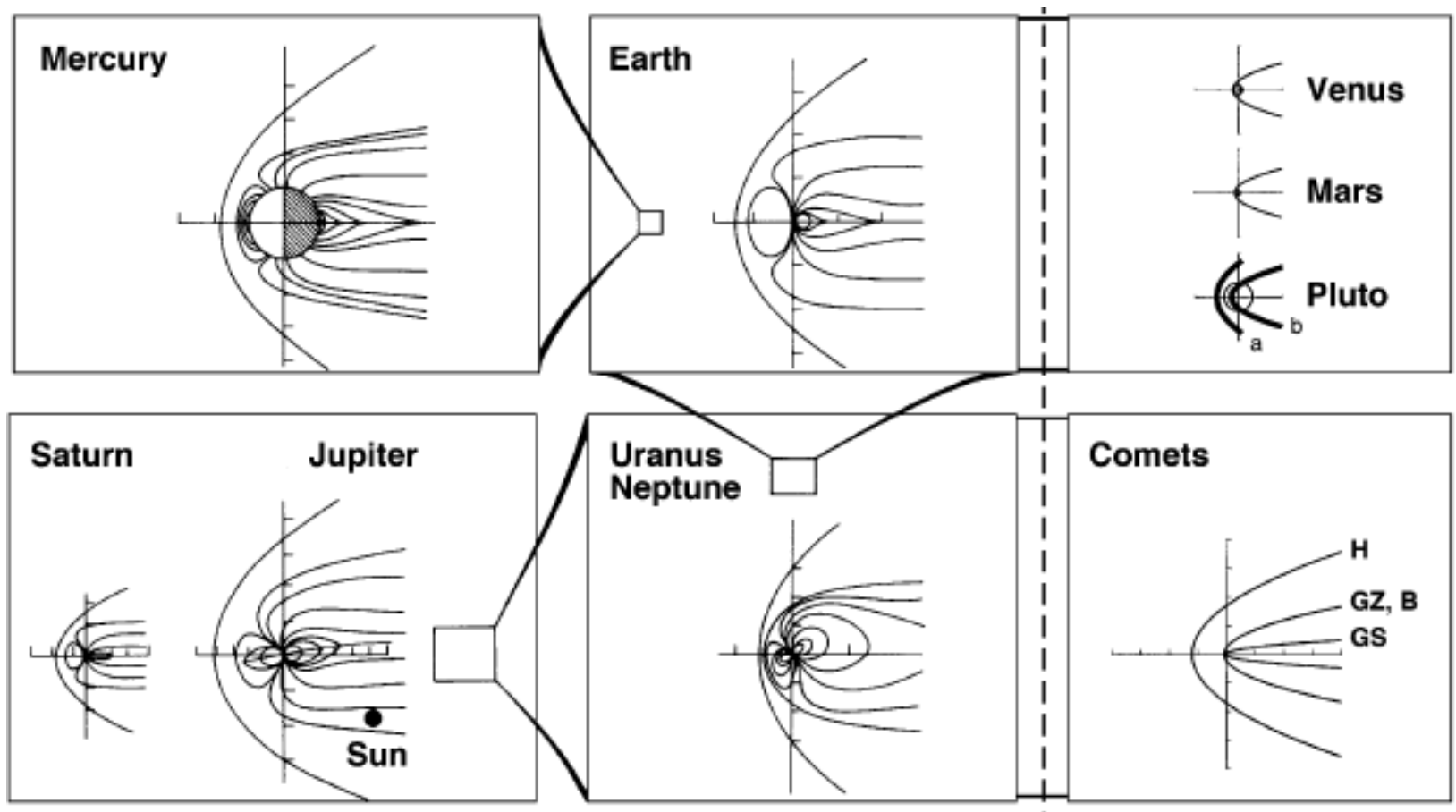

Figure 2.9.: Comparisons of the sizes of the solar wind interaction regions (magnetospheres) of various planets and comets. Taken from [Coates, 2004]. 


\section{Modelling Spacecraft Thruster Pickup Ions}

\subsection{Introduction}

Solar Probe Plus (SPP) is a NASA mission to investigate the near solar environment and will make its closest approach within ten solar radii. During perihelion SPP will realise velocities of up to $200 \mathrm{kms}^{-1}$ and therefore will have stringent pointing requirements in order that the science payload and spacecraft body remain within the shadow of the heatshield. As a result thruster firings will be necessary to correct for this either with small, frequent manoeuvres or with longer burns to de-spin the reaction wheels which are constantly operating in order to compensate for small adjustments to attitude. The propulsion subsystem of SPP consists of a blowdown mono-propellant hydrazine system consisting of twelve 0.9-N and two 4.4$\mathrm{N}$ thrusters. The propellant and pressurant are stored in the same tank, separated by a diaphragm so that as propellent is expended the pressure of the pressurant will decrease which will result in a lower thrust and specific impulse over the mission lifetime. Specific impulse is a measure of the efficiency of an engine and is the impulse produced per unit mass of propellant consumed. When measured in seconds it is equal to the duration an engine must fire in order to use an amount of propellant with its weight equal to the thrust produced. When the thruster valve is opened the helium pressurant acts to force the hydrazine from the tank and into the thruster where the hydrazine undergoes a catalytic reaction and decomposes into molecular hydrogen, molecular nitrogen and ammonia. Thence it expands through a nozzle thereby producing thrust.

Thruster firings in Earth orbit can produce significant quantities of neutral exhaust which then expands into a large cloud. Spacecraft in Low Earth Orbit (LEO) typically have velocities of approx $7.8 \mathrm{kms}^{-1}$ and those in geosynchronous orbit have velocities of approx $3 \mathrm{kms}^{-1}$. The exhaust velocity of a hydrazine thruster is approximately $3 \mathrm{kms}^{-1}$ and therefore the exhaust velocity is significant compared to 


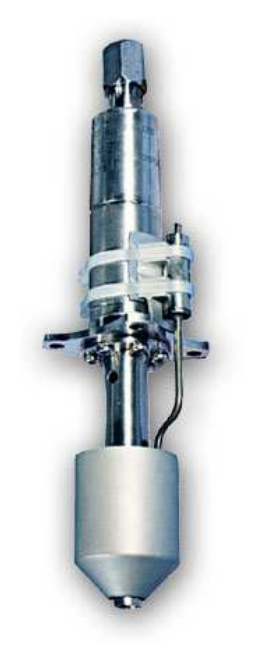

Figure 3.1.: Example of a 1N Hydrazine thruster.

Credit: Astrium [http://cs.astrium.eads.net]

the spacecraft velocity and the direction of thruster firing becomes important when considering the relative velocities of the solar wind flow and the spacecraft thruster plume. For this reason the major issues associated with thruster firings at $1 \mathrm{AU}$ are contamination of the spacecraft and potential damage to delicate sensors due to such sources as neutral plume backflow and impingement as well as any effects, such as sputtering, which come about as a result of charge exchange (CEX) ions. The majority of studies concerning thruster firings in the past have concentrated on the flow characteristics within the thruster nozzle and in the near field in order to predict the backflow onto the spacecraft or to identify the likely density of CEX ions. Typically these studies have a view to inform the engineers in advance of potential design issues and not so much the subsequent interactions of the thruster exhaust with any ambient plasma such as the solar wind or the earth's magnetosphere. In the context of spacecraft operations these effects are usually regarded as short term and minimal.

Most of these simulations use the direct simulation Monte Carlo (DSMC) technique which solves the Boltzmann equation probabilistically and is an efficient method of evolving collisional or collisionless flows. The concern mainly with backflow and plume impingement is justified due to the relative similarity in the magnitude of velocities of the spacecraft and the thruster exhaust at 1 AU. Very close to the sun, however, with spacecraft velocities of the order of $200 \mathrm{kms}^{-1}$ an exhaust velocity of $2-3 \mathrm{kms}^{-1}$ becomes far less influential in the overall motion of the neutral cloud in the solar frame although the relative motion of the spacecraft and the cloud remains the same. This is because the main factors which would influence the 
relative velocity and interactions of the cloud are the cloud's velocity with respect to the solar wind flow. If the solar wind flow is measured in hundreds of $\mathrm{kms}^{-1}$ as is the spacecraft velocity then a perturbation of only a few $\mathrm{kms}^{-1}$ can be considered almost negligible and hence the direction in which the spacecraft performs a thrust will not significantly affect this relative motion. Whilst the thruster exhaust is present near to the spacecraft at $1 \mathrm{AU}$ scientific data taking is typically reduced or halted completely so that perturbed readings are excluded and so that sensors which are particularly sensitive to ion flux can be shielded from the temporary increase.

For the case of Solar Probe Plus we are not only interested in the effects in the region near to the spacecraft but also in the larger scale interaction of the ions produced from the neutral cloud with the ambient plasma environment. For this reason we have developed a simulation code to predict the evolution of a neutral plume produced by a generic thruster firing. The DSMC technique, whilst effective, is not particularly well suited to this purpose due to the large size of the system being considered and the more extensive computational resources that would be required for such a program. Although a simulation based on the DSMC algorithms would yield an acceptable level of accuracy the number of macroparticles required to ensure that accuracy over such a large domain and hence the run time would be prohibitive, thus an alternate method was used, which is described later in this chapter.

Broadly speaking there are two main types of spacecraft thruster in use today: electric thrusters and chemical thrusters. The most basic type of electric thruster is the electrostatic ion thruster. This operates by injecting propellant into the discharge chamber which is then ionised via electron bombardment to form an electrically charged plasma. These electrons can be produced by a variety of methods such as microwave heating, RF emission or from a hollow cathode (Kaufman type) [Jugroot and Harvey, 2000]. The newly ionised plasma then diffuses towards the accelerating grids and is accelerated due to the potential difference between them. At this stage the accelerated ions pass through an ion-optics system which focuses the beam of plasma appropriately. The positively charged ion beam is kept electrically neutral by injecting an electron beam, called a neutraliser, into the ion beam. External plasma is prevented from back-streaming into the discharge chamber by the negative potential of the second grid although electrons can back-stream if the potential is not sufficiently negative. Figure 3.2 demonstrates an overview of the operation of a Kaufman type electrostatic ion thruster.

Electrostatic ion thrusters have a high specific impulse $(\approx 3000$ s) and benefit from a highly controllable thrust as well as a long, reliable operational lifetime but can 


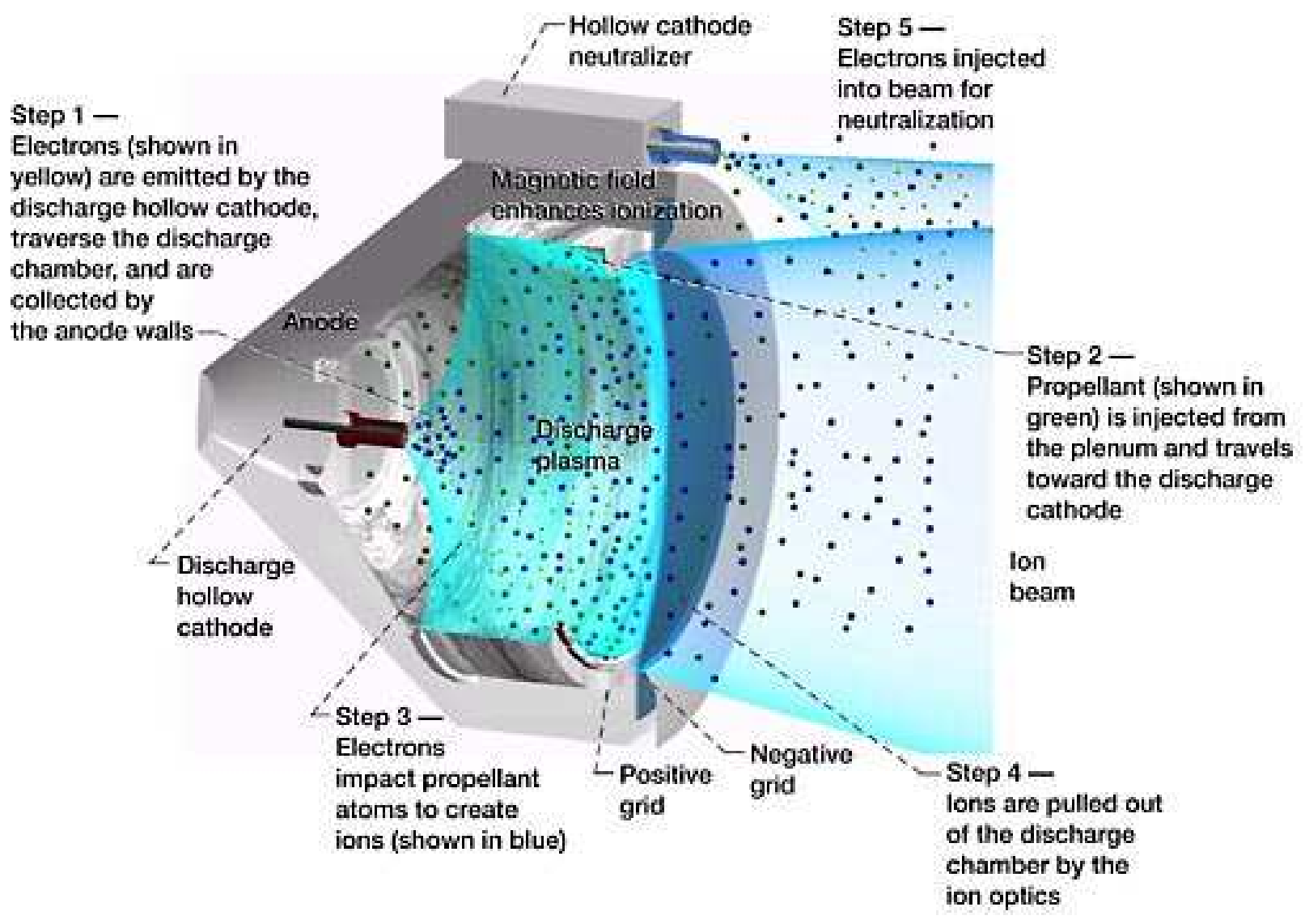

Figure 3.2.: Overview of the operation of a Kaufman type electrostatic ion thruster. Credit: NASA 
suffer from contamination issues and are restricted to use in a vacuum environment. As the function of the ion optics is not perfect due to a small range of possible exit velocities and the potential for multiple ionisation of the propellant the grids that form the ion optics are continually bombarded by a small amount of ionised propellant and are therefore eroded and worn away. This results in the production of a small amount of low energy secondary ions and neutrals. The low energy neutrals can collide with ambient plasma in a charge exchange reaction and form a low energy ion and fast neutral pair. If the potential of the spacecraft is sufficient then these low energy ions can be attracted back to the spacecraft and contaminate sensors, solar panels and other sensitive surfaces. Additionally these low energy ions have the potential to electrically connect exposed conducting surfaces of the spacecraft and thereby cause unintended electrical anomalies to occur.

In a Hall effect thruster the propellant (usually xenon but krypton may have potential use for high power thrusters due to its higher molecular mass) is injected through a distributor grid in the anode. Whereas in an ion thruster the propellant is ionised by direct bombardment with electrons, in a Hall thruster this task is accomplished by having a magnetically confined electron plasma at the thruster mouth with which the propellant collides after leaving the discharge chamber where an axial electric field then accelerates the ions. The combination of this radial magnetic field and the axial electric field causes the electrons to drift, forming the Hall current for which this thruster is named. The beam is neutralised via a neutralising cathode similar to the electrostatic ion thruster but Hall effect thrusters also deflect a portion of this electron beam to populate the ionising electron plasma ring. Figure 3.3 schematically shows the components and operation of a Hall thruster. Hall thrusters operate at high specific impulses $(\approx 2000$ s) although less than electrostatic ion thrusters. This is made up for by the fact that Hall thrusters have a higher thrust to power ratio and thus requires less power. Whilst the throughput of a Hall thruster is typically higher than an electrostatic ion thruster its lifetime in terms of operational hours is shorter although the total impulse produced can be comparable over their respective lifetimes. Hall thrusters can also suffer from the same issues and drawbacks as electrostatic ion thrusters.

Field-emission electric propulsion (FEEP) thrusters are a type of advanced electric thruster which use liquid metal, typically indium or caesium, as the propellant. The liquid metal is fed through a capillary which ends in a sharp point and is positively biased with respect to an electrode at the end with an electric field between them. At a sufficient strength of electric field the atoms at the tip of the capillary become ionised and are then accelerated by the electric field generated by the extraction 


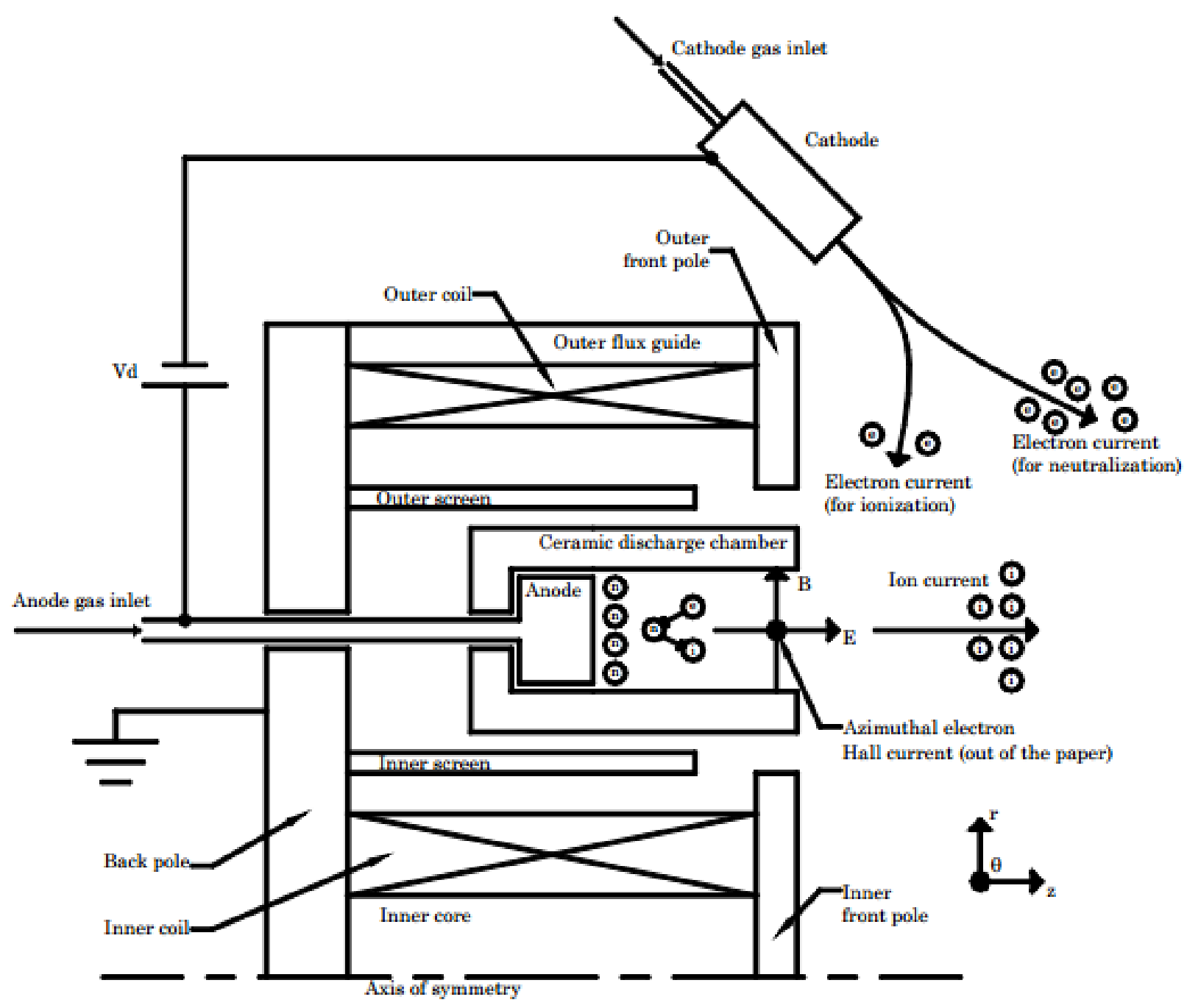

Figure 3.3.: Overview of the operation of a single stage magnetic layer Hall thruster. Credit: NASA 


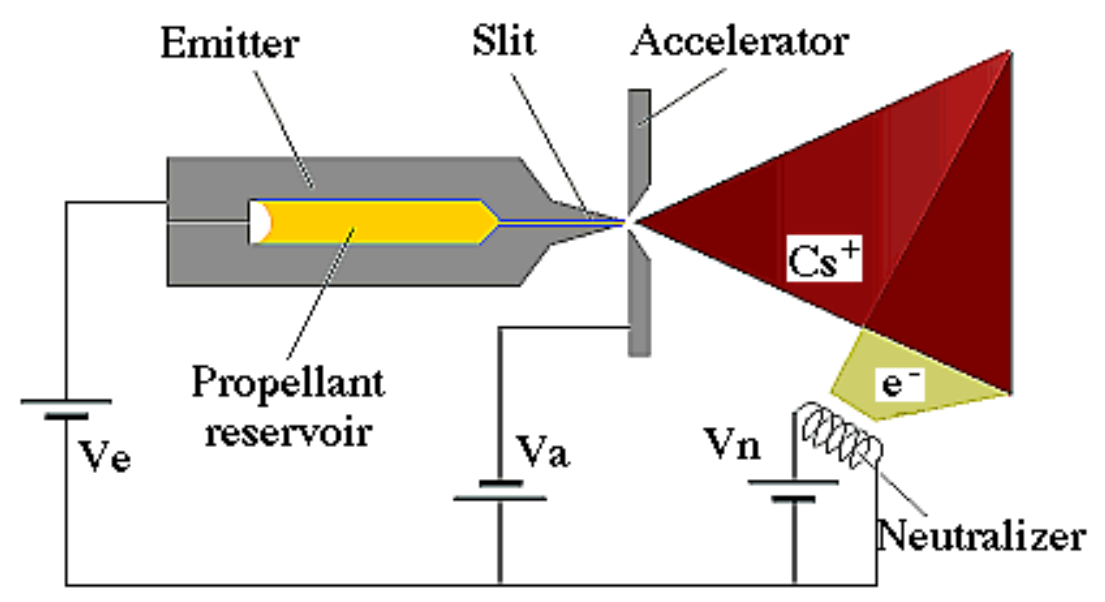

Figure 3.4.: Schematic diagram of the operation of a field-emission electric propulsion (FEEP) thruster.

Credit: ALTA-Space

electrode, this forms the beam of ions which produces thrust. This is illustrated in Figure 3.4.

Again, FEEP thrusters have a very high specific impulse $(\approx 10000$ s $)$ but the thrust produced by a FEEP thruster is generally much lower than either Hall or electrostatic ion thrusters; micro Newtons compared to milli Newtons. Unlike the previous types of electric thruster the FEEP thruster suffers from much larger ion beam divergence due to its relatively short acceleration path. FEEP thrusters also suffer from ion back-flow as do most electric thrusters but since the propellant is liquid metal in this case the back-flow will stick to the surfaces of the spacecraft where in other cases the contaminating ions can neutralise and leave the surface once more.

There is a large literature on the modelling and simulation of electric thrusters, studying generic problems such as plasma instabilities or the operation of specific thruster designs. For example, Tajmar [2002] created a model for Hall and ion thrusters in order to fully characterise the plasma environment induced which would allow prediction of the influence on optical sensors, the degradation of solar arrays and hence the effect on the spacecraft's overall lifetime. The model developed was a three-dimensional hybrid Particle-In-Cell code with Monte-Carlo collisions (PICMCC), it treats the ions and neutrals as test particles. The code can also simulate several different thruster-spacecraft geometry configurations and was validated by using ion current and energy measurements from three different thruster types. Similarly Cheouxdamas et al. [1997] developed software to determine the effects of thermal fluxes, force loading and torques as a result of plume impingement of ion 
thrusters and effects on solar arrays. Chanrion [2001] also used a numerical model in order to study the charging of a spacecraft in geostationary orbit using a stationary plasma thruster (SPT).

For some electric thrusters such as the electrostatic ion thruster the physics internal to the thruster can be important. Jugroot and Harvey [2000] performed simulations of the main chamber of a Kaufman-type ion thruster initially using a Navier-Stokes continuum code. The results of this code showed a sharp drop in pressure a short distance from the hollow cathode which indicated a rarefied flow, thus the Navier-Stokes code was only valid within the main chamber and a DSMC code was required to continue the simulation further away from the thruster nozzle. Additionally a qualitative description of the flow details within the main chamber was obtained which showed that the ionised particles were globally diffuse and that there were regions of enhanced ionisation and space charge related effects in the main chamber.

The earliest type of chemical thruster was the solid-fuel rocket and, like the hybrid rocket which uses a combination of solid and liquid propellant, is primarily used as a launch thruster rather then for manoeuvres due to having far higher thrust but lower specific impulse when compared to electric thrusters. More commonly used for orbital manoeuvres are the mono-propellant (monoprop) thrusters as briefly mentioned at the beginning of this chapter, bi-propellant (biprop) thrusters which operate on very similar principles to mono-propellant thrusters but will usually consist of a fuel and an oxidiser and liquid propellant rockets. As one moves from solid rockets through hybrids to liquid, monoprop and biprop thrusters the thrust decreases but the specific impulse of the thruster increases. An additional alternative is the very simple cold gas thruster, this operates by merely storing a pressurised propellant and produces thrust by opening a valve and expelling the gas. This has the benefit of being extremely simple and reliable but due to the gas being unheated the exhaust velocity, and hence performance, is very low. Due to the lower specific impulses of chemical thrusters compared to electric thrusters the lifetimes of these is often lower and the mass of propellant for a given impulse will be higher but they are required if a relatively large delta $\mathrm{V}$ is needed in a short time interval.

With respect to plasma interactions the effects of the solid and hybrid rockets can be ignored, they are generally not used in environments where it will be an issue and so the focus lies with the liquid, monoprop and biprop types of thruster. The issues that each face are very much the same in that the firing of the thruster will disturb the environment near the spacecraft which can result in contamination of the spacecraft due to the neutral plume or also in plume impingement which could 


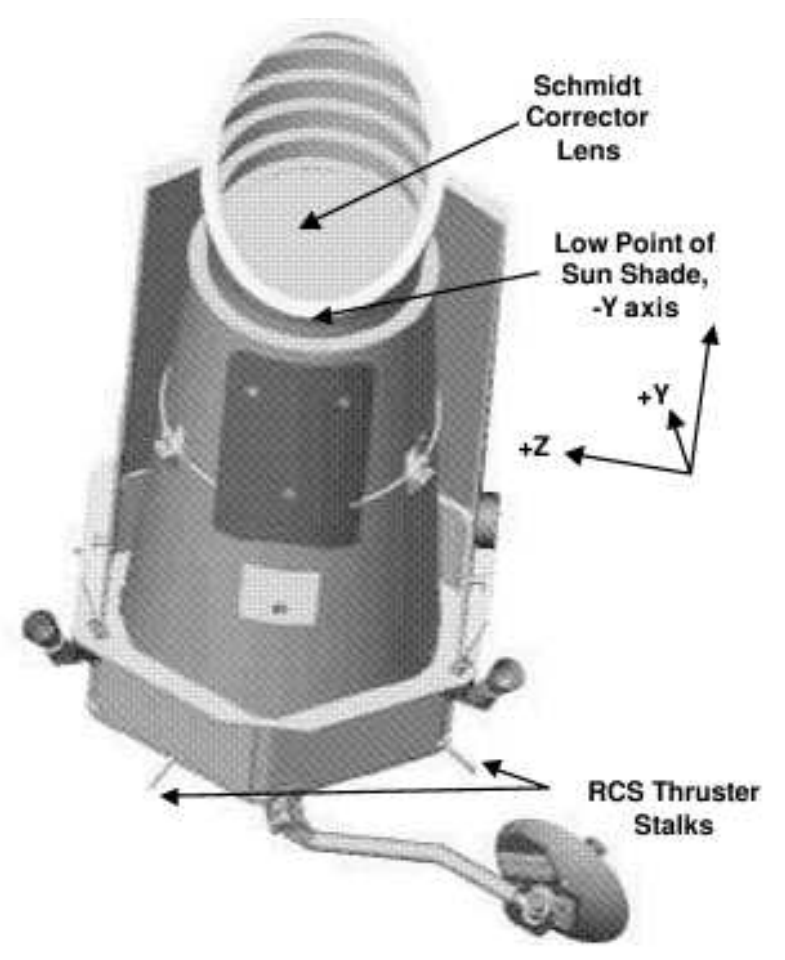

Figure 3.5.: Kepler flight system showing the photometer optics (Schmidt Corrector Lens).

Credit: Sholes et al. [2004]

cause force loading, thermal loading, erosion and chemical contamination.

Current research surrounding chemical thrusters addresses mainly the issues of contamination and plume impingement and also the calculation of the flow field surrounding the thrusters. For example, Sholes et al. [2004] performed a study to determine whether the hydrazine RCS blowdown system used on Kepler would cause contamination of the photometer optics as contamination could compromise pointing accuracy. Figure 3.5 shows the photometer optics and thus how easily the lens may become contaminated in unfavourable conditions. This was achieved by the use of several numerical simulations which used Navier-Stokes routines to determine the thruster internal analysis and a DSMC simulation to calculate the flow characteristics in the near-field. The conclusion was that the levels of contamination to the photometer optics would not reach unacceptable levels as a result of using hydrazine monopropellant thrusters.

A study of the various effects of plume impingement was carried out by Bury and Kerslake [2008] with the aim of determining whether the effects of a thruster firing might have a significant effect on the solar array power production of the Orion service module. They concluded that thermal loading and Paschen discharges may 


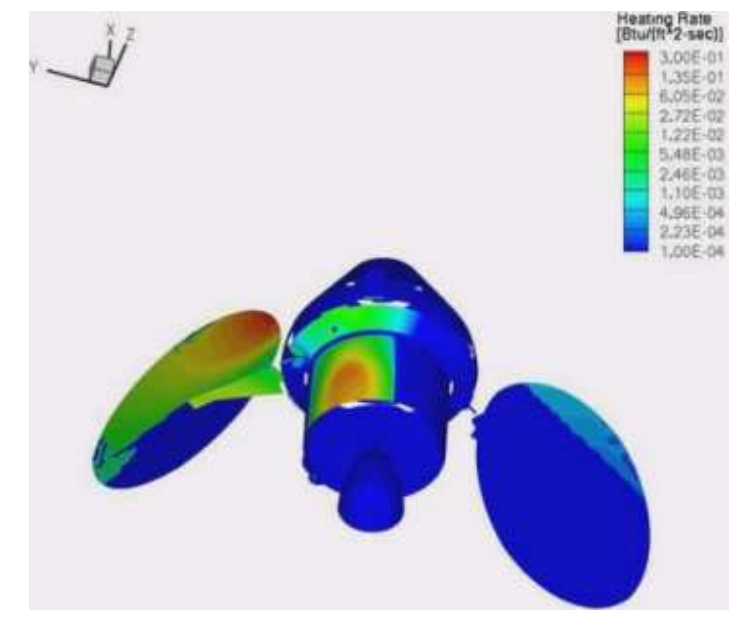

Figure 3.6.: Worst case scenario heat flux from one aft facing thruster on the Orion service module.

Credit: Bury and Kerslake [2008]

become an issue if the thrusters are allowed to fire for too long at any one time in the worst case scenario but that the effects of surface erosion and chemical contamination on the power production were well below the assumed plume impingement and induced power loss.

Hunton and Machuzak [1994] investigated the potential contamination of the Space Shuttle due to thruster firings where the magnetic field of the Earth was aligned such that the exhaust ions were 'picked up' and deflected back towards the Space Shuttle. They found that even the relatively small vernier reaction control system manoeuvring thrusters caused a significant perturbation of the ambient plasma but that the effects directly on the spacecraft depended largely on the firing angle with respect to the ram direction. Firings into the ram caused a decrease of local plasma ram flux by a factor of 50-100 whereas firings in the anti-ram direction do not cause significant changes to the local plasma ram flux. Firings at intermediate angles, however, did sometimes increase flux which they attributed to a collisional scattering mechanism.

\subsection{Neutral Expansion}

A simulation code has been produced to recreate the collisionless expansion of a neutral plume from a generic thruster firing in a vacuum. The simulation is performed in a $2 \mathrm{D}$ axisymmetric geometry with the thruster centreline lying along $\theta=0$ with respect to the $\hat{x}$ axis.

The neutral expansion is modelled in the frame of the spacecraft thruster and 


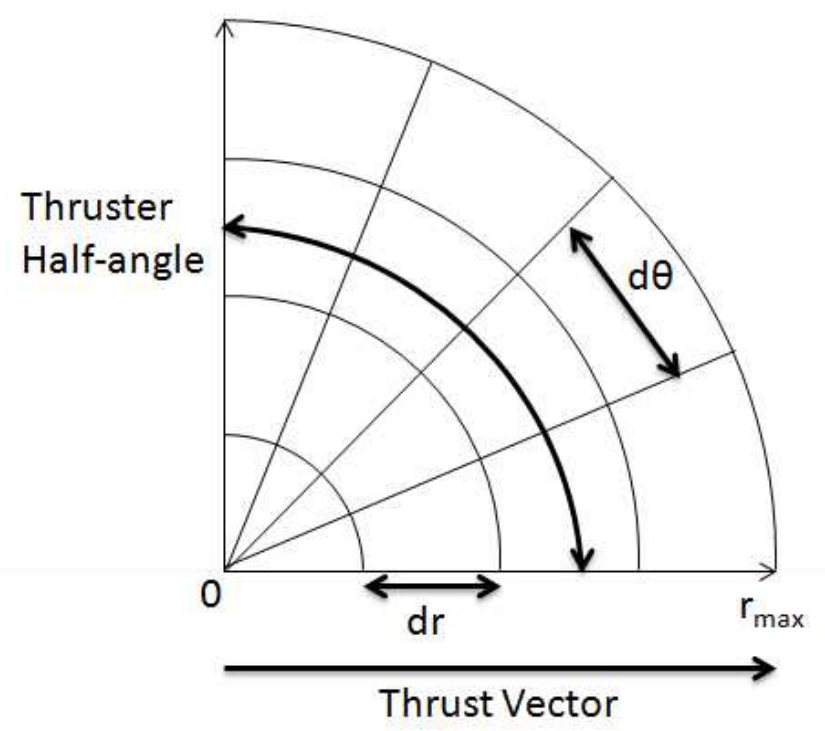

Figure 3.7.: Schematic diagram of the primary neutral mesh.

considers a single component of the neutral exhaust per run of the code where a component is a major molecular species present in the neutral plume after the burn, in the case of hydrazine $\mathrm{H}_{2}, \mathrm{~N}_{2}$ or $\mathrm{NH}_{3}$. As the flow is assumed to be collisionless (justified later in the chapter) this does not result in any additional error in the expansion of the neutral plume as the components would not interact significantly and it merely forces a slightly longer runtime. The neutral model begins by calculating an initial density of the form:

$$
n(r, \theta)=\frac{n_{0}}{r^{2}} \frac{\cos (\theta)}{e^{3 \theta}}
$$

Theta is in radians, $\mathrm{r}$ is in metres and $\mathrm{n}_{0}$ is the 'seed' value in $\mathrm{m}^{-3}$. This functional form is intended to represent the number density of the neutral thruster plume at the instant the thruster is turned off in a region near to the thruster nozzle. It is assumed to have captured in some sense the important aspects of the collisionality of the exhaust at these early times after which the assumption of collisionless flow can be invoked for the plume propagation. This form was chosen to be primarily axially directed and have a dependence on $\theta$ with an exponential falloff at the edges of the angle cone. The $1 / r^{2}$ term captures the near radial expansion at early times. The 'seed' value is proportional to the total mass of the burn and controls the magnitude of the neutral density at later times.

As the neutral material decomposes it reaches high temperatures and that thermal energy is then converted into a cold, directed flow as it passes through the thruster nozzle. The propulsion system on Solar Probe Plus consists of mono-propellant 


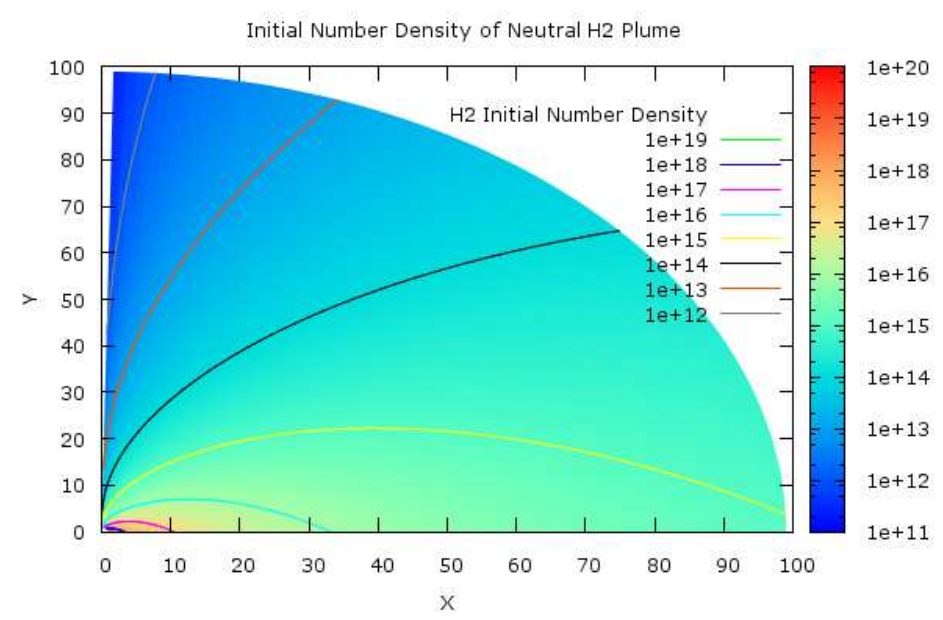

Figure 3.8.: Initial density profile of the $\mathrm{H}_{2}$ component of the thruster exhaust at $\mathrm{t}=0$.

hydrazine thrusters. The combustion chamber of such a thruster can quickly reach temperatures of 1500-2000K. Based on this and assuming an exhaust composition of $50 \% \mathrm{H}_{2}, 23 \% \mathrm{NH}_{3}$ and $27 \% \mathrm{~N}_{2}$ the average mass of a particle will be $\approx 2 \times 10^{-26} \mathrm{~kg}$. In order to determine the exhaust velocity profile we can make some considerations of the energetics involved. The energy of a particle at $1750 \mathrm{~K}$ will be:

$$
\begin{aligned}
E & =\frac{3 k_{b} T}{2} \\
& =3.62 \times 10^{-20} \mathrm{~J}
\end{aligned}
$$

and hence the resulting bulk velocity will be:

$$
\begin{aligned}
v & =\sqrt{\frac{2 E}{m}} \\
& =1903.3 \mathrm{~ms}^{-1}
\end{aligned}
$$

This leads to velocity and temperature distributions which are easily justifiable based on the energetic considerations above of:

$$
\begin{gathered}
T_{0}(\theta)=10+0.4 \theta \\
u(\theta)=1200+600 \cos (\theta)
\end{gathered}
$$




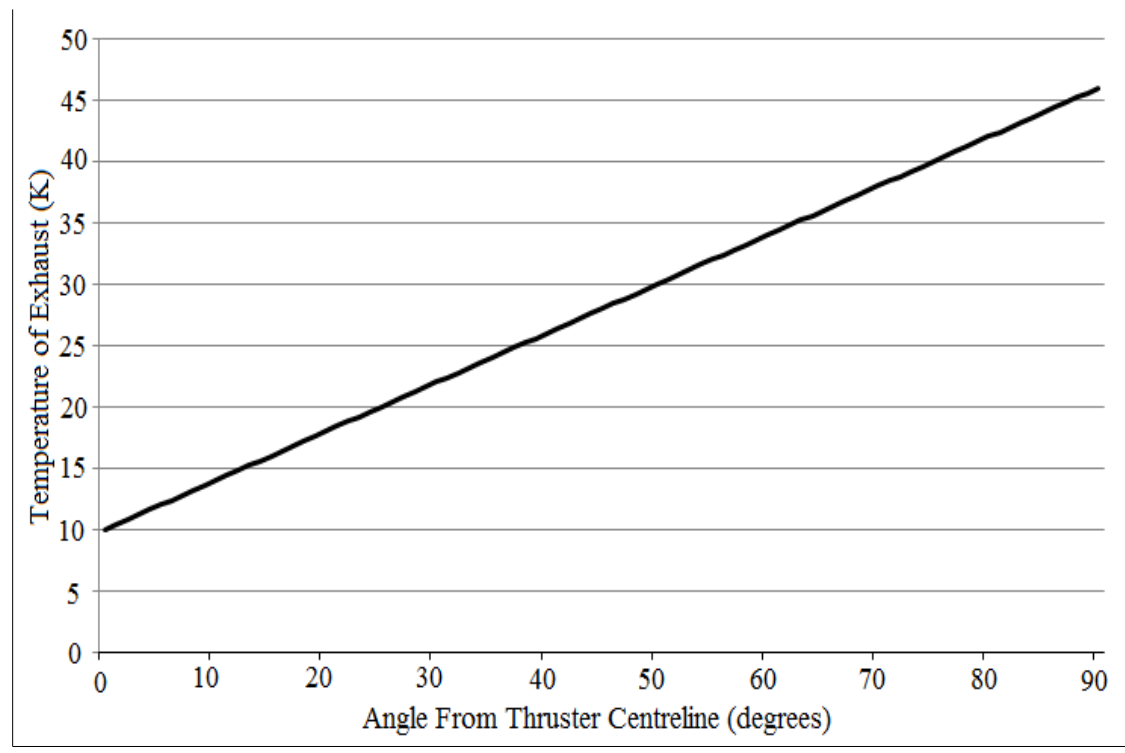

Figure 3.9.: Exhaust temperature as a function of angular distance from the thruster centreline as per eqn 3.2 .

for $0 \leqq \theta \leqq 90$ with $\theta$ measured in degrees and temperature in Kelvin. The functions of $\theta$ have been chosen to give what might be expected from the diverted flow of the exhaust gas. The evolution of the neutral plume is calculated assuming a Maxwellian distribution and free molecular expansion into a vacuum in a $2 \mathrm{D}$, axisymmetric geometry about the thruster centreline/thrust vector. Figure 3.11 shows an example hydrazine thruster with an example of how the thrust vector relates to the nozzle geometry.

The mean free path for particles with a Maxwellian distribution from kinetic theory is:

$$
\lambda=\frac{1}{\sqrt{2} n \sigma}
$$

Where $\sigma$ is the hard-sphere collision cross section and $\mathrm{n}$ is the neutral number density. Since the mean free path can be considered to vary as $(n \sigma)^{-1}$ and using the collision cross section of $\mathrm{N}_{2}$, which is larger than that of $\mathrm{H}_{2}$, of $\sigma=7 \times 10^{-20} \mathrm{~m}^{-2}$ and a number density of $10^{20} \mathrm{~m}^{-3}$ we find that the mean free path is $0.1 \mathrm{~m}$ at $1 \mathrm{~m}$ along the thruster centreline and since the number density goes as $1 / r^{2}$ at $10 \mathrm{~m}$ along the thruster centreline the mean free path is $10 \mathrm{~m}$ and at $100 \mathrm{~m}$ along the thruster centreline the mean free path is $1000 \mathrm{~m}$. Thus once beyond the initial plume the collisionless flow assumption is reasonable. 


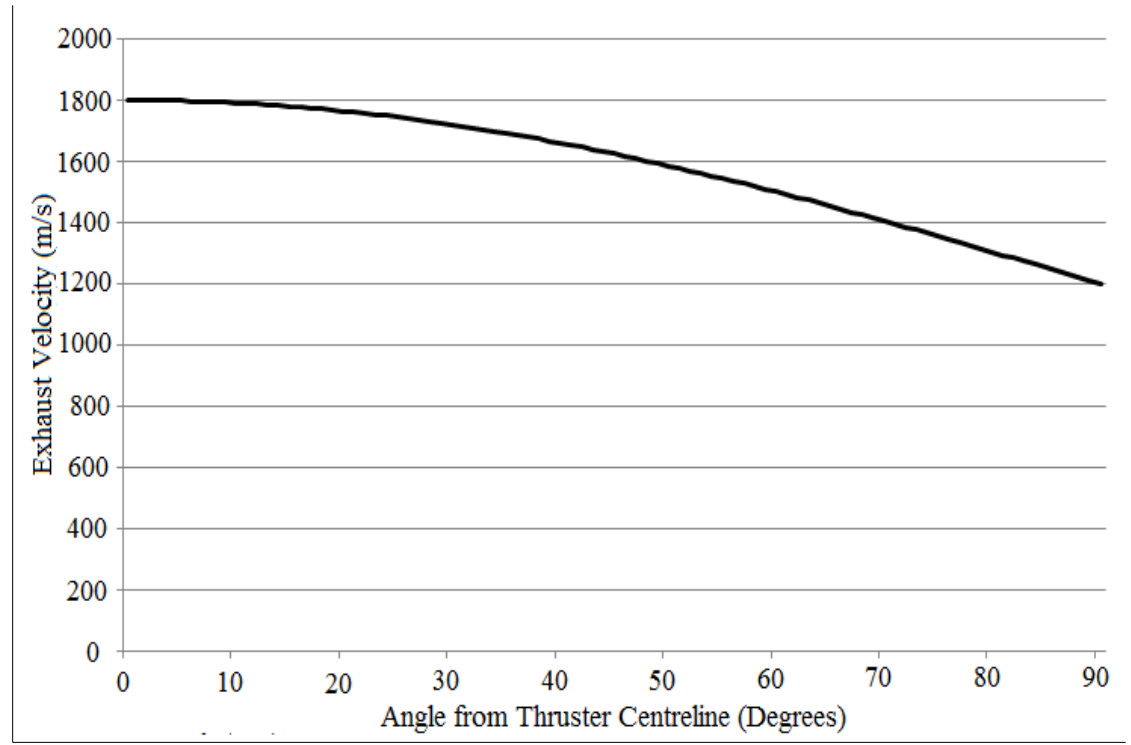

Figure 3.10.: Exhaust velocity as a function of angular distance from the thruster centreline as per eqn 3.3.

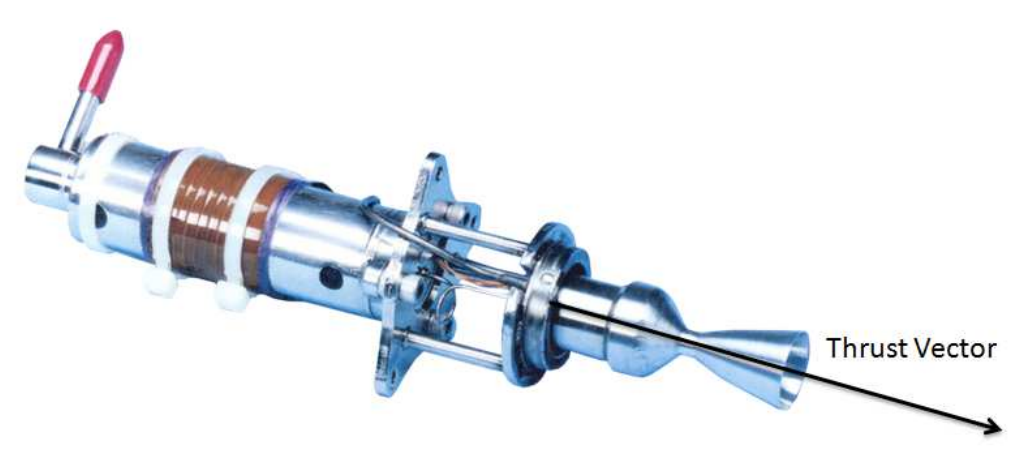

Figure 3.11.: 5N Hydrazine thruster showing the definition of thrust vector/thruster centreline used.

Base picture credit: Aerojet Rocketdyne, Inc. 


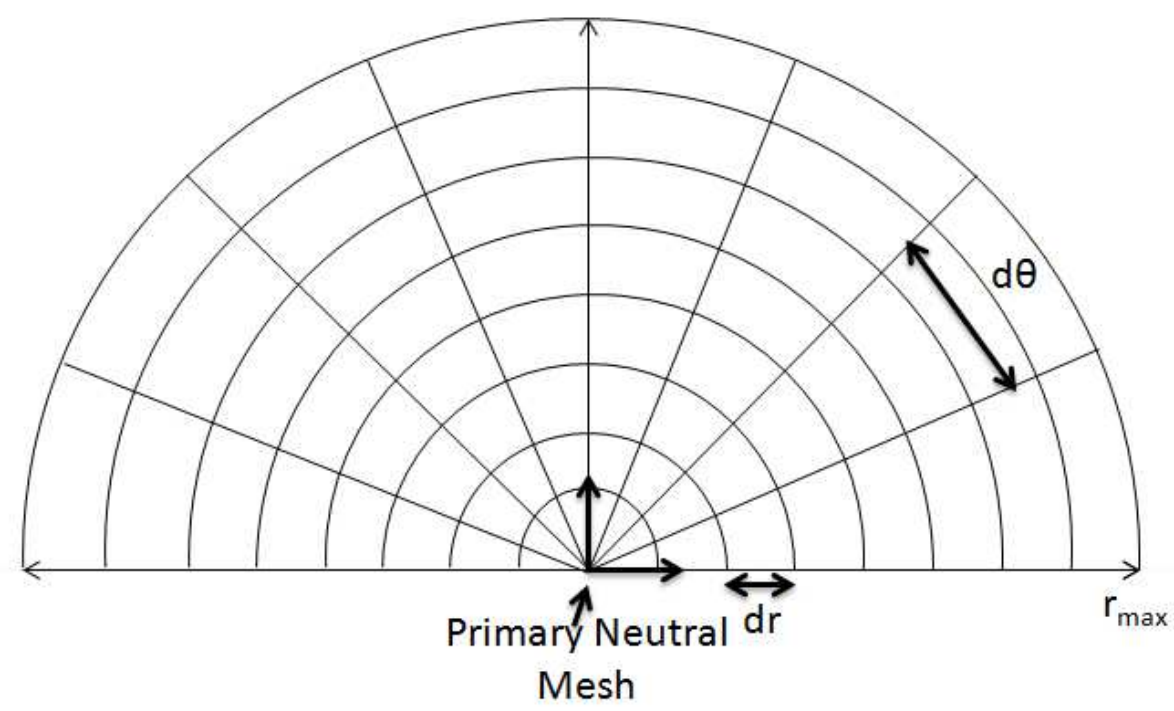

Figure 3.12.: Schematic diagram of the secondary neutral mesh and ion mesh. The primary mesh is small compared to the secondary mesh.

The actual expansion of the neutral plume is modelled by creating a primary mesh over the initial number density and expanding each cell individually onto each cell on a much larger secondary mesh. See Figures 3.7 and 3.12 for details of the construction of the primary and secondary meshes. The number density in each cell of the secondary mesh is comprised of the sum of the contributions of number density from every cell in the primary mesh at each time step by:

$$
\begin{aligned}
F\left(r, \theta, r_{0}, \theta_{0}, t\right)= & \frac{V_{r_{0}}}{V_{r}} n_{0}(r, \theta) \sqrt{\frac{m_{s}}{2 \pi k_{b} T_{0} t^{2}}} \\
& \exp \left(-\left(\frac{m_{s}}{2 k_{b} T_{0}\left(\theta_{0}\right) t^{2}}\right)\left(\left(d x-u_{x}\left(\theta_{0}\right) t\right)^{2}+\left(d y-u_{y}\left(\theta_{0}\right) t\right)^{2}\right)\right)
\end{aligned}
$$

Where:

- $d x=r \cos (\theta)-r_{0} \cos \left(\theta_{0}\right)$

- $d y=r \sin (\theta)-r_{0} \sin \left(\theta_{0}\right)$

- $u_{x}=u_{0} \cos \left(\theta_{0}\right)$

- $u_{y}=u_{0} \sin \left(\theta_{0}\right)$

- $V_{r_{0}}=\frac{\pi d r}{135}\left(3 r_{0}^{2}+3 r_{0} d r+d r^{2}\right)$ 


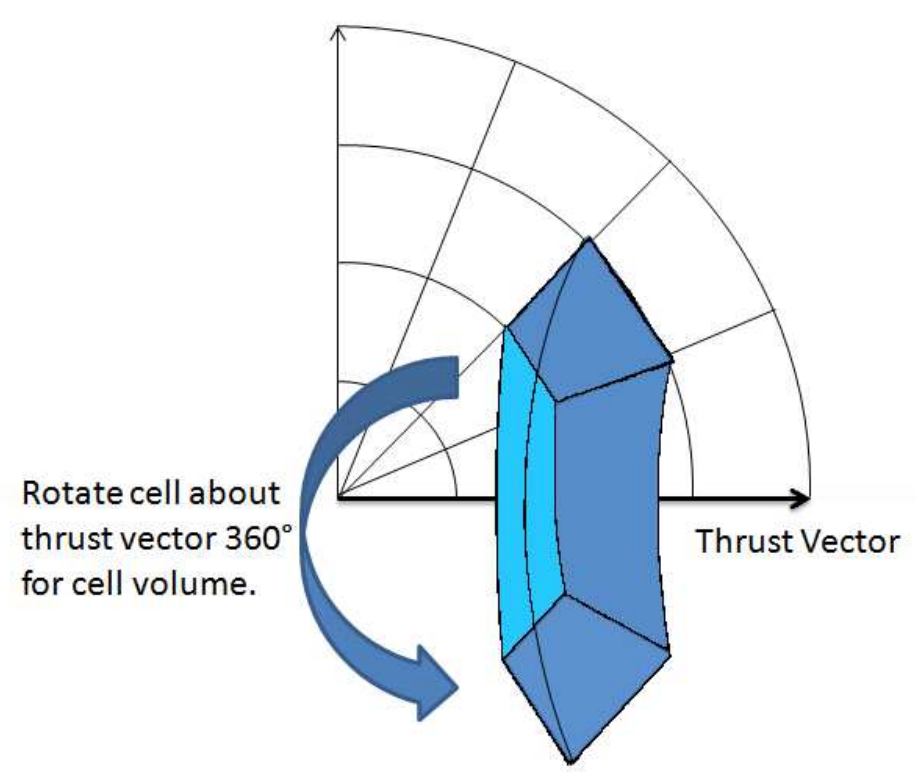

Figure 3.13.: Illustration of the volume an axisymmetric cell sweeps out.

- $V_{r}=\frac{\pi d r}{135}\left(3 r^{2}+3 r d r+d r^{2}\right)$

- $m_{s}$ is the mass of the species in question

- $k_{b}$ is Boltzmann's constant

$\mathrm{V}$ is the volume of a cell in the mesh rotated about the thrust vector (see Figure $3.13)$ and is generally:

$$
V_{r}=\frac{4 \pi d \theta}{3}\left((r+d r)^{3}-r^{3}\right)
$$

This neutral expansion technique builds on works by Cai and Boyd [2007], Cai and Boyd [2007], Cai and Wang [2013], Narasimha [1962] which assumes a Maxwellian velocity distribution from which particle number densities can be calculated at all points across a secondary mesh at some later time after the thrust assuming collisionless propagation of velocities.

In each cell of the secondary mesh the only particles which can contribute to the number density there are those in the primary mesh which have a velocity which lies within $v=\frac{\Delta r \pm 2 d r}{t}$ where $\Delta r$ is the distance between the cell centres, $d r$ is the average cell width along the line joining the two cells. This is because for a specified start and end location with respect to the cells under consideration only particles with a specific velocity will have travelled that distance after the specific amount of time has elapsed. It is equivalent to stating that only particles with 


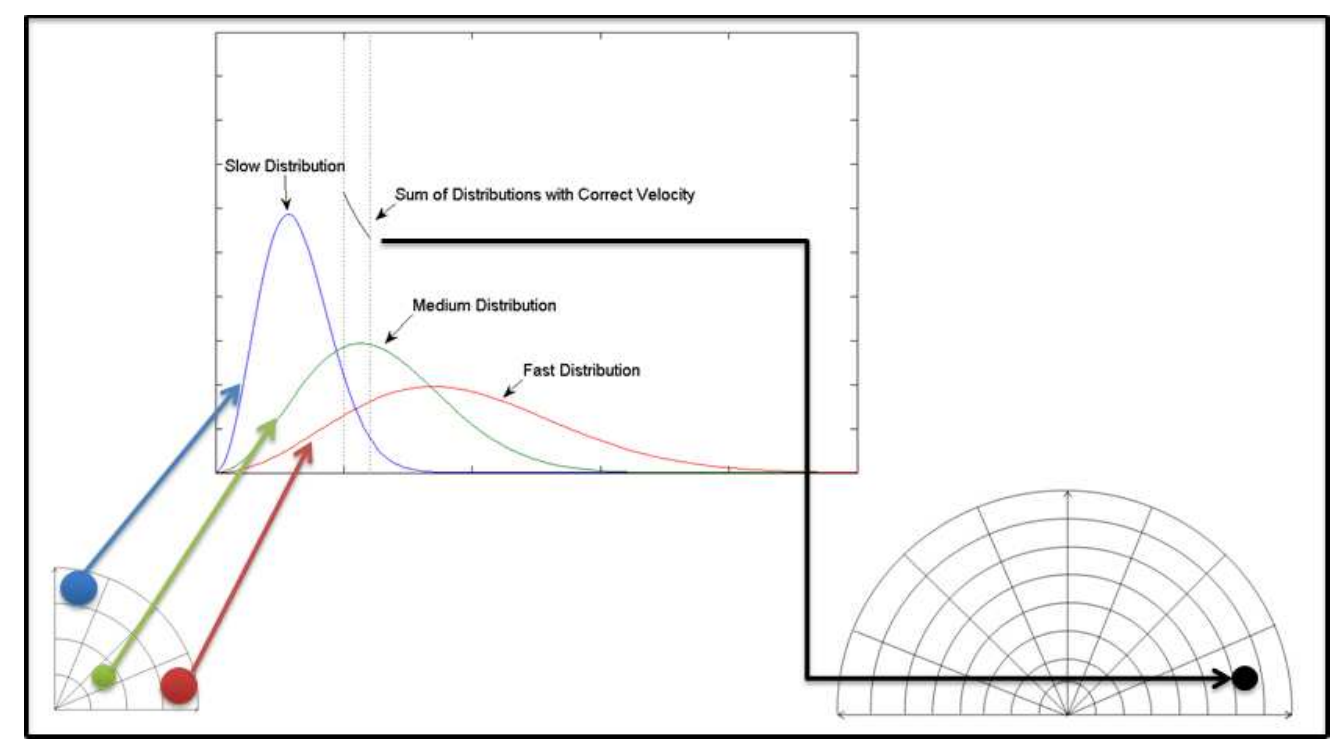

Figure 3.14.: Schematic diagram of contributions by the primary mesh to a cell in the secondary mesh.

a particular velocity will have travelled this particular distance by this particular time. The stringency of this velocity requirement is relaxed as the cell sizes increase and as the time step increases. For cells in the primary mesh with different velocity distributions but which are physically located close together then the contributions each give to a single cell located far away will be the particles located in the same part of their distribution function, the sum of which will be contributed to the cell in the secondary mesh. This is, again, a result of the requirement that particles have a particular velocity in order to travel a particular path in a given amount of time. A schematic diagram of this concept is illustrated in Figure 3.14 with the velocity requirement relaxed in sufficient proportion to the size of a cell. In this figure three different example distributions in primary mesh cells located physically close together are given along with the resulting, combined distribution which satisfies the conditions necessary to be located in the cell in the secondary mesh which is assumed to be located far enough away from the three primary mesh cells that the differences in distance are negligible. Each individual mapping of the particle distribution from $\mathrm{t}=0$ to any later time, $\mathrm{t}$, will obey Liouville's theorem, which states that the phase space distribution function is incompressible, as there are no boundaries present in the code and the flow is collisionless as well as there being no external forces acting upon the neutrals. Whilst particles are lost to ionisation, this is captured in the recalculation of the initial density mesh and hence the actual expansion process continues to satisfy the conditions necessary.

Therefore with the number density in any cell of the secondary mesh being a sum 
of the contributions of each cell from the primary mesh we obtain an expression for number density of a secondary mesh cell at time, t:

$$
n(r, \theta, t)=\sum_{r_{0}=0}^{r_{0 \max }} \sum_{\theta_{0}=0}^{\phi} F\left(r, \theta, r_{0}, \theta_{0}, t\right)
$$

Where:

- $r, \theta$ is the secondary mesh cell the number density is being calculated for

- $r_{0}, \theta_{0}$ is the primary mesh cell being considered

- $r_{0 \max }$ is the maximum radial distance of the primary mesh

- $\phi$ is the thruster half-angle

As an example Figures 3.15 and 3.16 show the evolution of the $\mathrm{H}_{2}$ and the $\mathrm{N}_{2}$ plumes separately at the simulation time of 28.5 seconds, that is, 28.5 seconds after the thruster is assumed to have stopped firing at a distance of ten solar radii. The solar wind number density is assumed to be approximately $10^{9}$ particles per cubic metre for comparison and the figures are in the frame of the spacecraft. These represent small burns of only a few grams in total. The general shape of the neutral plume produced is what would be expected as the flow is compressed slightly in the ram direction (along the $\hat{x}$ axis) and with the leading edge of the profile curving backwards for increasing $\theta$ as the flow velocity decreases. Due to the assumption of a Maxwellian velocity distribution it is possible for some of the slower moving particles to expand in such a way that backflow occurs and envelops the spacecraft as a result of their thermal velocities being comparable to, or greater than, their flow velocity. This is far more evident for the hydrogen component than for the nitrogen as the higher mass of the nitrogen causes it to have a much lower thermal speed and hence less spread compared to the hydrogen. Additionally it can be seen that the core of these clouds moves with the appropriate velocity of several $\mathrm{kms}^{-1}$. 


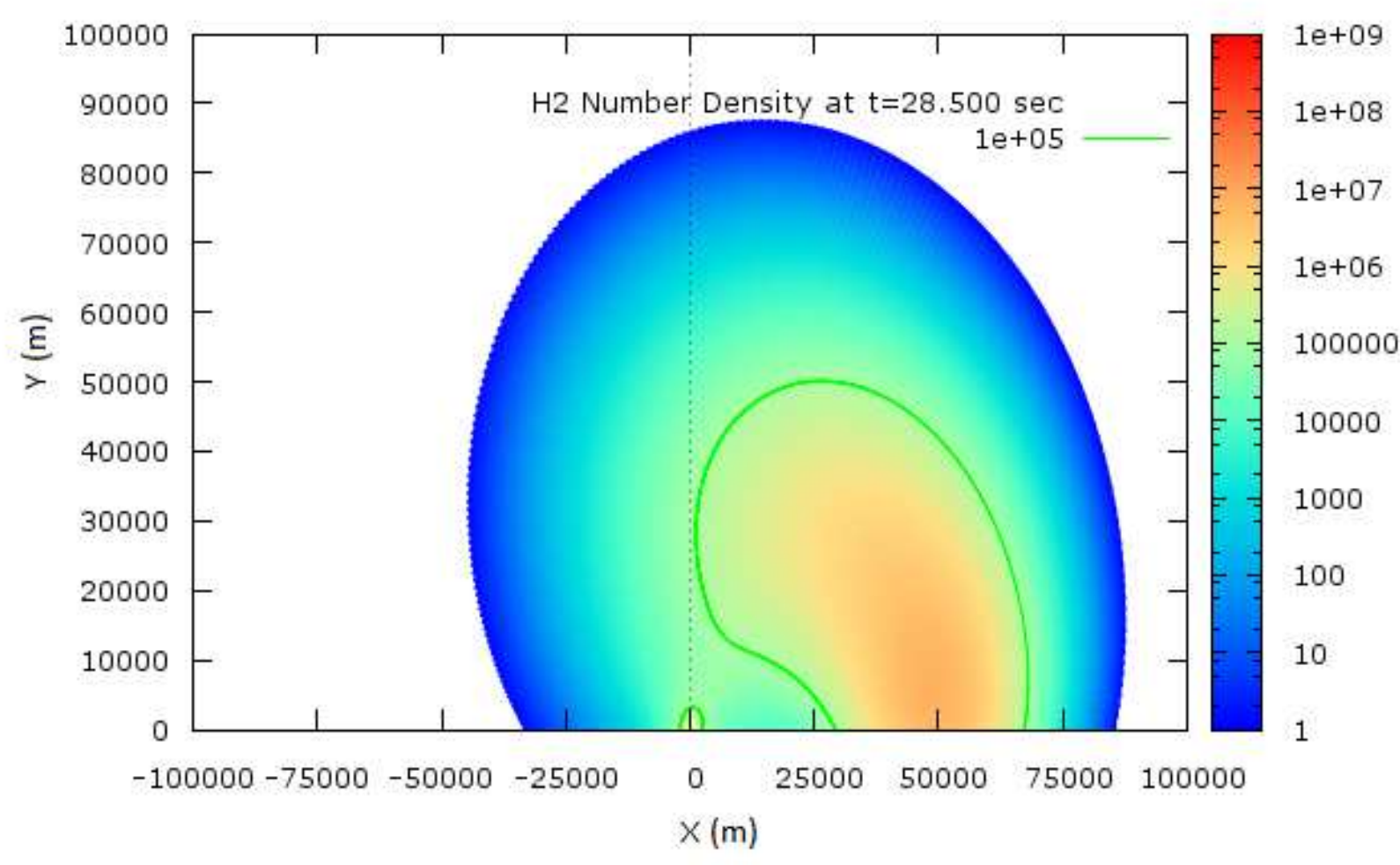

Figure 3.15.: $\mathrm{H}_{2}$ plume number density at $28.5 \mathrm{sec}$ in $\mathrm{m}^{-3}$. 


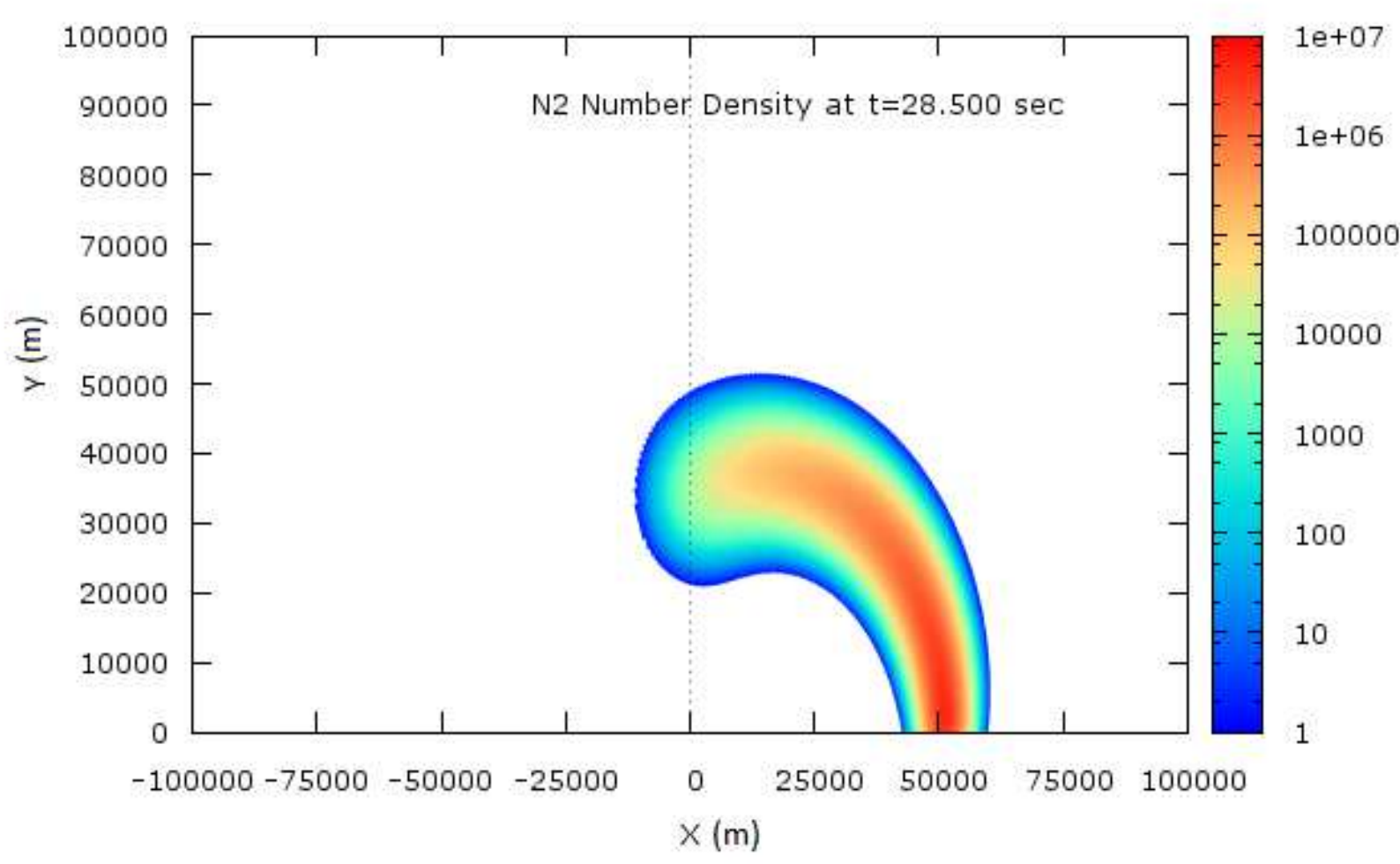

Figure 3.16.: $\mathrm{N}_{2}$ plume number density at $28.5 \mathrm{sec}$ in $\mathrm{m}^{-3}$. 


\subsection{Ionisation}

The ion plume is created on a new mesh identical in structure to the secondary neutral mesh and is done by assuming a fraction of the total neutral plume is ionised equally throughout its volume per time step. This equates to an assumption that the neutral cloud is optically thin in the UV region. The amount of neutrals ionised per time step is calculated by using the ionisation rates either measured or calculated for processes at $1 \mathrm{AU}$ and scaling them back to the required solar distance as $1 / r^{2}$. Ionisation rates were taken from Siscoe and Mukherjee [1972]. Only certain ionisation processes are considered and these include several ionisation pathways for $\mathrm{H}_{2}$ and $\mathrm{N}_{2}$. Specifically they are, for $\mathrm{H}_{2}$, the photolysis of $\mathrm{H}_{2}$, the photolysis and ionisation of $\mathrm{H}_{2}$, the photo-ionisation of $\mathrm{H}_{2}$, ionisation due to the impact of solar wind electrons with $\mathrm{H}_{2}$, ionisation due to the impact of solar wind protons with $\mathrm{H}_{2}$, ionisation due to charge exchange with solar wind protons and the photo-ionisation of $\mathrm{H}$.

$$
H_{2}+h \nu \rightarrow H+H
$$

Photolysis of $\mathrm{H}_{2}$.

$$
\mathrm{H}_{2}+\mathrm{h \nu} \rightarrow \mathrm{H}^{+}+\mathrm{H}+\mathrm{e}^{-}
$$

Photolysis and ionisation of $\mathrm{H}_{2}$.

$$
H_{2}+h \nu \rightarrow H_{2}^{+}+e^{-}
$$

Photo-ionisation of $\mathrm{H}_{2}$.

$\mathrm{H}_{2}+e^{-} \rightarrow \mathrm{H}_{2}^{+}+2 e^{-}$

Impact of solar wind electrons with $\mathrm{H}_{2}$.

$\mathrm{H}_{2}+\mathrm{H}^{+} \rightarrow \mathrm{H}_{2}^{+}+\mathrm{H}^{+}+\mathrm{e}^{-}$

Impact of solar wind protons with $\mathrm{H}_{2}$.

$\mathrm{H}_{2}+\mathrm{H}^{+} \rightarrow \mathrm{H}_{2}^{+}+\mathrm{H}$

Charge exchange of $\mathrm{H}_{2}$ with solar wind protons.

$H+h \nu \rightarrow H^{+}+e^{-}$

Photo-ionisation of $\mathrm{H}$.

Similarly for $\mathrm{N}_{2}$ the processes considered are the photolysis and ionisation of $\mathrm{N}_{2}$, ionisation due to the impact of solar wind electrons, photolysis of $\mathrm{N}_{2}$ and the photoionisation of $\mathrm{N}$. 
$N_{2}+h \nu \rightarrow N_{2}^{+}+e^{-}$

Photolysis and ionisation of $\mathrm{N}_{2}$

$N_{2}+e^{-} \rightarrow N_{2}^{+}+2 e^{-}$

Impact of solar wind electrons with $\mathrm{N}_{2}$

$N_{2}+h \nu \rightarrow 2 N$

Photolysis of $\mathrm{N}_{2}$

$N+h \nu \rightarrow N^{+}+e^{-}$

Photo-ionisation of $\mathrm{N}_{2}$

As each pass of the code evolves a different neutral component it must also similarly calculate the ionisation of the different neutral components on separate passes, this results in the different species being completely decoupled in this case. Components are handled separately in order to reduce the computational resources required but this does, however, allow each component of the fuel to be examined specifically and separately to the other components which provides a useful opportunity to categorise the bulk motion of the different mass ions. It should be noted that while there is plentiful experimental data available for the photolysis and ionisation rates of $\mathrm{H}_{2}$ at $1 \mathrm{AU}$ there is less when it comes to secondary ion production. There is less data available for the ionisation of $\mathrm{N}_{2}$ but it is still possible to provide rates which consider several of the major photolysis and ionisation pathways. There is almost no data available for the ionisation of $\mathrm{NH}_{3}$ and thus at this time it has not been included in simulations. As the ammonia ultimately ionises into nitrogen and hydrogen it is possible to approximate their ionisation by artificially enhancing the amount of the other species present if required. The ion plume is created by ionising a fraction of the neutral plume at each time step according to the above reaction equations on a new, separate mesh with the same ranges as the secondary neutral mesh, see Appendices A and B for more details. Additionally after a fraction of the neutral cloud is ionised the neutral density must be reduced accordingly to compensate for this loss of material. This is done by modifying the 'seed' value used to calculate the initial density and thereby creating an updated profile for the 'initial density' which is then expanded in the next time step to give a reduced number density at all points which represents the optically thin nature of the cloud as the ionisation occurs evenly throughout. This method has been tested against the analytic decay formula used and has been found to agree within $1 \%$. The new 'seed' 
value is calculated according to equation 3.8 .

$$
n_{0}(t+1)=n_{0}(t)[1-\lambda d t]
$$

Where:

- $\mathrm{n}_{0}(t+1)$ is the reduced seed value to be used in the next time step

- $\mathrm{n}_{0}(t)$ is the seed value used this time step

- $\lambda$ is the total rate at which $\mathrm{H}_{2}$ or $\mathrm{N}_{2}$ is ionised by all processes combined

- $\mathrm{dt}$ is the ionisation time step

\subsection{Implementation}

The program is written in $\mathrm{C}++$ and makes use of the key value format (KVF) third party library for the input parameter file.

The inputs to the program are:

\section{Thrust Vector}

The thrust vector is specified in the format $[\hat{x} \hat{y} \hat{z}]$ and is converted into a unit vector within the code and sets the orientation of the thrust vector in the $3 \mathrm{D}$ test particle section of the code.

\section{Solar Wind - B Angle}

The angle between the solar wind flow vector and the ambient magnetic field is set by this input. The magnetic field is defined to lie along the $\hat{x}$ axis. This angle therefore affects the direction of the solar wind velocity vector and hence that too of the motional electric field.

\section{Solar Distance}

This input, in units of solar radii, specifies the distance from the Sun at which the simulation takes place and causes a host of other parameters, such as the magnetic field strength, the spacecraft velocity, the solar wind number density, the solar wind velocity and the ionisation rates for the different species to be set.

\section{Destination Folder}

This input specifies where the output files will be located. This path is relative to the directory the neutral code was executed from. 


\section{Species}

This input determines which component of the hydrazine fuel will be simulated in this run. The different components are separated out to reduce the memory usage per individual simulation. This does not introduce additional error as the different species are assumed to not interact in the neutral phase and cannot interact in the test particle phase.

\section{Test Particle Simulation}

This input determines whether or not the simulation will create and move the macroparticles or if it will just compute the neutral and ionised meshes.

\section{Density Threshold}

This input defines where the edge of the neutral cloud is assumed to be, it determines which cells will have densities too low to be considered to be properly part of the neutral cloud and are hence included in calculating an average density for the cloud. This input is not critical to the successful operation of the code.

\section{Number of Burns}

This input determines the total number of 'burns' that will take place in the simulation. A single 'burn' corresponds to a very short ( $0.15 \mathrm{~s})$ burn of a fixed mass. The mass flow rate is comparable to that of a typical hydrazine thruster. This allows the user to either burn for a selected duration or to burn a selected amount of hydrazine. Unfortunately the user must determine the number of these burns that correspond to their chosen value manually. Fractional burns are possible but any fractional burn is always the final burn of a simulation and thus the mass flow rate cannot be changed at this time.

\section{Maximum Secondary Angle}

This input determines the maximum radial extent of the secondary mesh but will usually be set to $180^{\circ}$ for most simulations.

\section{Ionisation Scale Factor}

This input scales the ionisation rates such that the neutral plume will be ionised more quickly or more slowly. This is of use if the solar distance the hybrid simulation is being performed at has a cyclotron time which is very short, thus a more rapid ionisation shows the behaviour when a larger fraction of the neutral cloud has been ionised. 


\section{Burn Separation}

This input determines the time in seconds between successive burns if a longer duration burn is being approximated by a composite burn.

The primary neutral mesh typically has a maximum radius of $100 \mathrm{~m}$ and a thruster half angle of $60^{\circ}$. This half angle is somewhat large for a hydrazine thruster but the specific half angle used has been found to have a negligible effect on the evolution of the neutral plume within the assumptions and limits of this model over the long term. The secondary neutral mesh as well as the ion meshes have a maximum radius of $100 \mathrm{~km}$ and extend over the full $180^{\circ}$. The time step used is $0.025 \mathrm{~s}$ for a total duration of $30 \mathrm{~s}$. The radial cell width used is $50 \mathrm{~m}$ and the angular width used is $1^{\circ}$.

A potentially large limitation to the code is the fact that the neutral mesh is fixed on the spacecraft means that at some point in the simulation the neutral plume will effectively 'fall off' the edge of the mesh and so all data past that time is invalid for a sufficiently long duration simulation. This can be compensated for by sizing the mesh appropriately for the duration of the simulation to be performed by considering the plume's bulk velocity. This has a consequence of increasing the number of operations required to calculate the neutral plume evolution and hence increasing the runtime of the code exponentially.

\subsection{Hybrid Model}

The plasma interaction between the ambient solar wind and the newly born thruster ions is modelled by a hybrid simulation. Hybrid simulations are characterised by modelling the ions as particles and the electrons as a massless, charge neutralising fluid. The hybrid code suffers from being unable to simulate the 'near-field' environment around the spacecraft where the densities are initially highest due to cell size resolution and therefore there will be errors associated with this but the hybrid code does capture larger gyro-scales. Similarly as electrons are modelled as an adiabatic fluid the hybrid code is unable to capture details of electron interactions and the high frequency waves associated with them. We use the 3D code HYPSI which is based on the CAM-CL algorithm by Matthews [1994]. This uses the moment method to advance the velocity or current density half a time step ahead and then collects the source terms (plasma density and currents) in order to solve for the fields. Once the fields are found then the particles are advanced once more and new source terms are calculated. 
The Maxwell equations include Poisson's Law:

$$
\nabla \cdot \vec{E}=\frac{\rho}{\epsilon_{0}}
$$

Gauss's Law:

$$
\nabla \cdot \vec{B}=0
$$

Faraday's Law:

$$
\nabla \times \vec{E}=-\frac{\partial \vec{B}}{\partial t}
$$

and Ampere's Law:

$$
\nabla \times \vec{B}=\mu_{0} \vec{J}+\frac{1}{c^{2}} \frac{\partial \vec{E}}{\partial t} .
$$

In the hybrid simulation Poisson's Law is replaced by the fluid momentum equation as the electrons are treated as a charge neutralising, massless fluid, hence we can eliminate some terms, rearrange to obtain the electric field and use Ohm's Law:

$$
\vec{E}=-\vec{V}_{e} \times \vec{B}-\frac{\nabla P_{e}}{e n_{e}}+\eta e n_{e}\left(\vec{V}_{i}-\vec{V}_{e}\right)
$$

Ampere's Law is used to eliminate $V_{e}$ in equation 3.13 and Faraday's Law is used to advance the magnetic field in time. An isothermal or adiabatic equation of state is also used. The ions, which have a charge and mass of $q_{i}$ and $m_{i}$ respectively, are kinetic and hence the equations of motion are solved:

$$
F=\sum_{i=1}^{3} m_{i} \frac{d \vec{V}_{i}}{d t}=\sum_{i=1}^{3} q_{i}\left(\vec{E}+\vec{V}_{i} \times \vec{B}\right)
$$

and

$$
\frac{d \vec{x}_{i}}{d t}=\vec{V}_{i}
$$

Where the sum, i, is over all species included in the simulation.

This separation of required scales allows the resolution of ion gyrokinetics whilst not requiring the much greater computational resources needed to resolve the electron gyrokinetics over the duration being simulated. Hybrid simulations have been widely used to study the pickup interaction of the solar wind with comets and planetary bodies (e.g., Cowee and Gary [2012], Cowee et al. [2008]).

The hybrid simulations performed using HYPSI are in a fully three-dimensional co-ordinate system in the frame of reference of the solar wind. The simulation uses fully periodic boundary conditions and therefore can suffer from periodic wraparound. This can be seen in our simulations near the edges of the simulation domain at late 
times in the simulation however, we are mostly interested in the central part of the simulation domain which is far away from where any of these effects are important.

Unlike in the neutral code where all values are in SI units, all values in the hybrid code are normalised. The number densities are normalised to the assumed ambient solar wind density. All velocities are normalised to the ambient (undisturbed) Alfvén speed. Lengths are normalised with respect to the proton inertial length. Time is normalised to the inverse cyclotron frequency. Magnetic field strengths are normalised to the ambient solar wind magnetic field strength. Mass and charge are both normalised to the values of a proton.

$$
\begin{aligned}
\tilde{V} & =\frac{V}{V_{A}} \\
\tilde{x} & =\frac{x}{\lambda_{i}} \\
\tilde{t} & =t \Omega_{i c} \\
\tilde{n} & =\frac{n}{n_{s w}} \\
\tilde{B} & =\frac{B}{B_{s w}} \\
\tilde{m} & =\frac{m}{m_{p}} \\
\tilde{q} & =\frac{q}{q_{p}},
\end{aligned}
$$

where the values with tildes denote normalised quantities and:

$$
\begin{aligned}
V_{A} & =\frac{B_{0}}{\sqrt{\rho \mu_{0}}} \\
\lambda_{i} & =\frac{c}{\omega_{i p}} \\
\omega_{p i} & =\sqrt{\frac{n_{s w} q_{p}^{2}}{m_{p} \epsilon_{0}}} \\
\Omega_{i c} & =\frac{q B_{0}}{m_{p}} .
\end{aligned}
$$

The hybrid code was modified to include multi-species capability and to allow the injection of additional ions taking the production rate from the neutral expansion code. In order to circumvent some computational restraints the neutral ionisation has been artificially enhanced such that all of the species ionise at a rate four times 
that which would be expected. This is so that over the duration of the simulation we can observe the effects of the ionisation of a more significant fraction of the neutral cloud. If allowed to ionise at its normal rate then a far larger simulation would be necessary in order to produce the same amount of ionised material, that is to say, both the neutral cloud and the ion cloud will be smaller and denser than in reality but of course this also depends on the amount of mass burned in any particular thruster firing.

The injection rates produced by the neutral code are on a $2 \mathrm{D}$ axisymmetric mesh in the spacecraft frame. This mesh is then rotated such that the $\hat{x}$ axis in the neutral code is aligned with a user chosen thrust vector in the hybrid geometry. Ions are then created rotated about that axis based on the injection rate for this time step, with the origin of the $2 \mathrm{D}$ mesh being placed at the location of the spacecraft in the hybrid code and all distance values of the cell locations being normalised according to the simulation being performed. 


\section{Interaction of Spacecraft Thrusters with the Solar Wind - An Overview}

\subsection{Introduction}

Spacecraft commonly use chemical thrusters for orbital and attitude manoeuvres. Thruster firings produce an exhaust plume of neutral molecules which then expands into a large cloud spanning hundreds of kilometres after only a few minutes for a sufficiently large burn. Effects of such thruster firings have been studied for a long time and can be broadly split into two categories: scientific issues and operational issues. The operational issues include plume backflow and interactions of the spacecraft with the induced plasma environment. Topics such as contamination of the spacecraft sensors and solar arrays must be addressed as well as potential transient spacecraft charging effects [Ergun et al., 2010, Marchand et al., 2014]. The thruster exhaust and subsequent products also create a relatively turbulent environment on their own and in most cases require either partial or full sensor shut down which can seriously affect data taking if this is required at a time when accurate measurements are critical. All of these things can lead to interactions of the spacecraft with the ambient plasma and have the potential to cause operational problems if not properly planned for. The scientific issues are mainly to do with measurements of the ambient plasma. The very presence of the plume will affect and modify the characteristics of the solar wind flowing around the spacecraft and, at least for the short term, will contaminate measurements of the ambient magnetic field and plasma. This is especially true if measurements of the solar wind are attempted during the actual thruster firing itself.

Ionisation time scales for the neutrals are of the order of several tens of minutes, so these effects have the potential to persist for relatively long periods of time and thus these interactions require some investigation in order to characterise the disturbances 
most likely to occur so that they may be filtered out of any scientific data which is taken. Alternatively a way might be found to identify the period of time during which the measurements are irretrievably contaminated by large scale disturbances as well as informing of how long any reduced data taking may be required to last. The motivation for this work is the Solar Probe Plus (SPP) NASA mission due to launch in 2018. Its closest approach to the sun will take it within ten solar radii of the surface of the sun where both the plasma environment and ionising conditions are very different from at $1 \mathrm{AU}$ where most space plasma measurements are made. Furthermore, because of thermal constraints Solar Probe Plus will require frequent attitude changes during perihelion and therefore some use of chemical thrusters is planned. In this chapter we present an overview of a simulation corresponding to a thruster firing of Solar Probe Plus performed at solar conditions similar to those that may be experienced at the spacecraft's perihelion of approximately ten solar radii. This work has been submitted to Physics of Plasmas as a paper titled "Pickup Ion Processes Associated with Spacecraft Thrusters: Implications for Solar Probe Plus" Clemens and Burgess [2016].

\subsection{Simulation Setup}

This simulation is a 'standard' thruster firing at $90^{\circ}$ to the initial, undisturbed magnetic field direction which will be used as a reference for comparison with subsequent simulations in the chapters to follow. Table 4.1 summarises the normalised simulation parameters and the corresponding assumed values in SI units for conditions predicted at ten solar radii. It has been calculated also that the total equivalent real mass injected into the system over the duration of the simulation is approximately $30 \mathrm{~g}$ with the bulk of that owing to the greater atomic weight of nitrogen, this would be typical of a medium duration burn (of the order of minutes). The hybrid simulations have a cell size of one proton inertial length and the total domain size is a cube with sides of $240 \lambda_{i}$. The time step used in the simulation is $0.02 \Omega_{i}^{-1}$ and the total duration of the simulation is $120 \Omega_{i}^{-1}$. Initially there are 300 macroparticles per cell representing the solar wind and zero thruster particles, the thruster particles are all injected throughout the simulation's duration at a rate which is not constant but increases with time. The fast solar wind velocity is taken to be $480 \mathrm{kms}^{-1}\left(1.39 V_{A}\right)$ directed perpendicular to the spacecraft's velocity of $190 \mathrm{kms}^{-1}\left(0.55 V_{A}\right)$ which gives a relative injection velocity of $517 \mathrm{kms}^{-1}\left(1.5 V_{A}\right)$. The ambient solar wind number density is taken to be $1 \times 10^{9} \mathrm{~m}^{-3}$ and the ambient magnetic field strength of 500 
Table 4.1.: Simulation Parameters In Normalised and Real Units

\begin{tabular}{ccc}
\hline \hline Parameter & $10 \mathrm{R}_{\odot}$ & $0.05 \mathrm{AU}$ \\
\hline Cell Width & $1.0 \lambda_{i}$ & $7200 \mathrm{~m}$ \\
Time Step & $0.02 \Omega_{i}^{-1}$ & $0.0026 \mathrm{~s}$ \\
Particles Per Cell & 300 & \\
Domain Size & $240 \times 240 \times 240 \lambda_{i}$ & $1728 \times 1728 \times 1728 \mathrm{~km}$ \\
Corrected Injection Velocity & $1.5 V_{A}$ & $517.5 \mathrm{kms}^{-1}$ \\
Magnetic Field Strength & 1.0 & $500 \mathrm{nT}^{-1}$ \\
Solar Wind Velocity & $1.39 V_{A}$ & $480 \mathrm{kms}^{-1}$ \\
Spacecraft Velocity & $0.55 V_{A}$ & $190 \mathrm{kms}^{-1}$ \\
Solar Wind Number Density & 1.0 & $1 \times 10^{9} \mathrm{~m}^{-3}$ \\
Duration & $120 \Omega_{i}^{-1}$ & $15.74 \mathrm{~s}$ \\
\hline
\end{tabular}

$\mathrm{nT}$ is assumed directed parallel to the solar wind flow which is also perpendicular to the spacecraft's velocity. The ions are injected with their velocity directed along the $+\hat{y}$ axis (i.e. the thrust is in the ram direction of the spacecraft) with the ambient magnetic field directed along the $+\hat{x}$ axis and the $\hat{z}$ axis completing the triad. Additionally, the spacecraft is assumed to travel in the direction of the thruster firing with a velocity of $517 \mathrm{kms}^{-1}\left(1.5 V_{A}\right)$ and hence will remain close behind the region in which the neutral cloud lies (and hence the injection region for this simulation).

\subsection{Results}

The simulation was carried out in the solar wind frame so it is important to note the following. Mass is loaded onto the solar wind by the introduction of ions as a result of the ionisation of the neutral thruster plume through various processes. As this mass is picked up by the motional electric field of the solar wind, the solar wind is itself slowed down locally in the solar frame of reference in order to conserve energy. In the simulation frame of reference the solar wind is accelerated and the injected ions are slowed down. As the magnetic flux is frozen to the solar wind flow it begins to pile up in the region ahead of the spacecraft where the solar wind flow speed is reduced the most due to energy losses to the newborn pickup ions (in the solar frame). This results in a pile up of the magnetic field lines such that there is a strong enhancement ahead of the spacecraft. This has the further effect that the field lines drape around the edge of the interaction region as the solar wind flow recovers further away from the spacecraft where the mass loading effects do not affect the flow to as great a degree. 

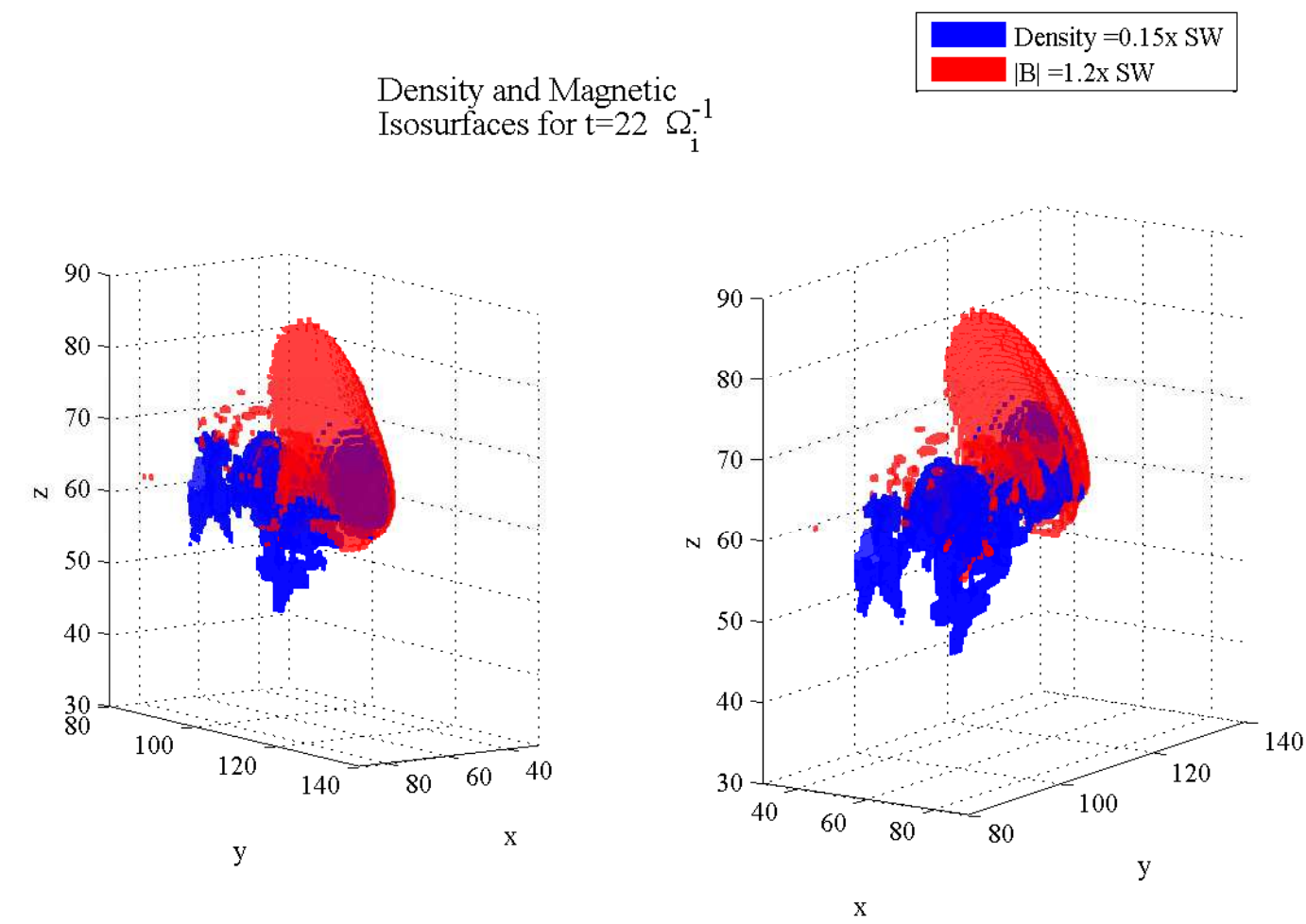

Figure 4.1.: Isosurfaces of the magnetic field strength and number density of injected material from two angles. The leading magnetic bow wave can be seen ahead of the ion cloud with ions being deflected in $+\hat{z}$ at that front and with some material being deflected in $-\hat{z}$ from the centre of the cloud.

This also results in the formation of a bow wave ahead of the spacecraft as the solar wind plasma is swept up by the bunched magnetic field lines. The combination of bow wave and the magnetic field pile up and draping forms an induced interaction region around the spacecraft which forms a barrier and, to some extent, constrains the interaction of the newborn ions with the solar wind.

Due to the injection of material causing the magnetic field lines to be pushed away from the spacecraft and the resultant sweeping up of the solar wind plasma, the induced interaction region has its ambient magnetic field reduced in the core area such that it approaches becoming diamagnetic. The field within the interaction region is mainly a self-generated field from the current of the newborn ions, without 
which the volume would be field free and diamagnetic in a similar way to the AMPTE 'comet' [Bernhardt et al., 1987, Harold and Hassam, 1991]. An additional indicator of this is that the electric field on a plane through the centre of the interaction region displays the same kind of outward-directed orientation as was observed by AMPTE as is displayed in figure 4.2 in the region of the bow wave near to the spacecraft. In this figure and all following figures the crosshair shows the position of the spacecraft within the simulation.
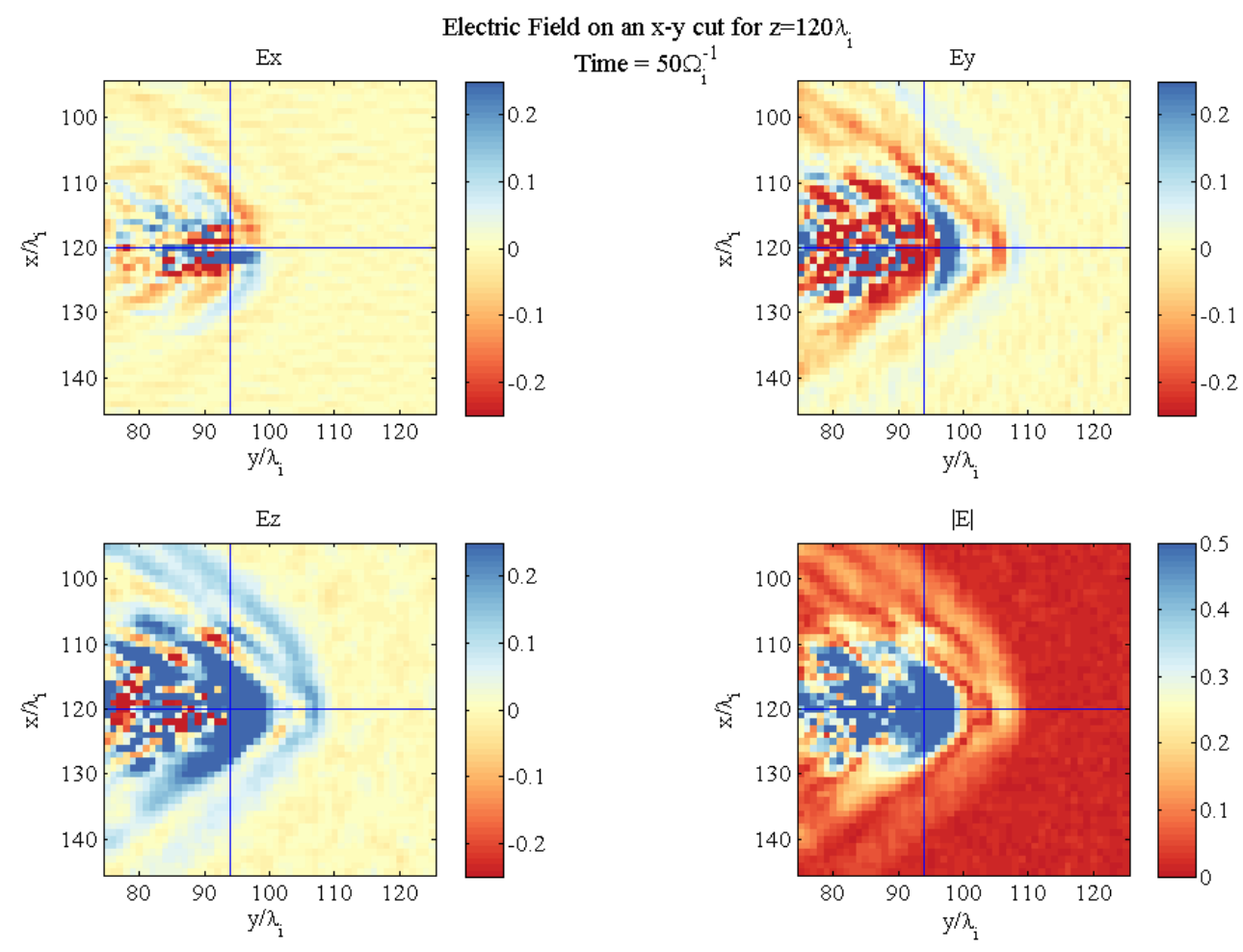

Figure 4.2.: A heatmap plot of the electric field on an $\hat{x}-\hat{y}$ plane through the centre of the simulation domain after $50 \Omega_{i}^{-1}$. This shows that at the leading edge of the interaction region that the field is polarised to be directed outward with respect to the core of the cloud. The $E_{z}$ component does not show the same polarisation in this figure as this is a cut through the $\hat{z}$ axis, it changes sign in a similar fashion upon moving to a different plane.

Figure 4.1 shows what happens as the interaction region begins to form and the types of structure that are seen. Plotted are isosurfaces for constant values of both magnetic field magnitude and injected number density, both panels show the same image but differing by view angle to provide a better perspective of the 3 -D structure and to highlight the shape of the 'shells' formed by the thruster firing. Ahead of the spacecraft the magnetic field pileup can be seen extending along $+\hat{z}$ and $\pm \hat{x}$ in a 
hemispherical shell with the injected material streaming behind it. Additionally in the $-\hat{z}$ direction some material can be seen. This is primarily the nitrogen component being deflected in 'clumps' as is shown by figure 4.3. Further behind the spacecraft the hydrogen component can be seen being caught on the field lines and being left behind in a more typical example of ion pickup.

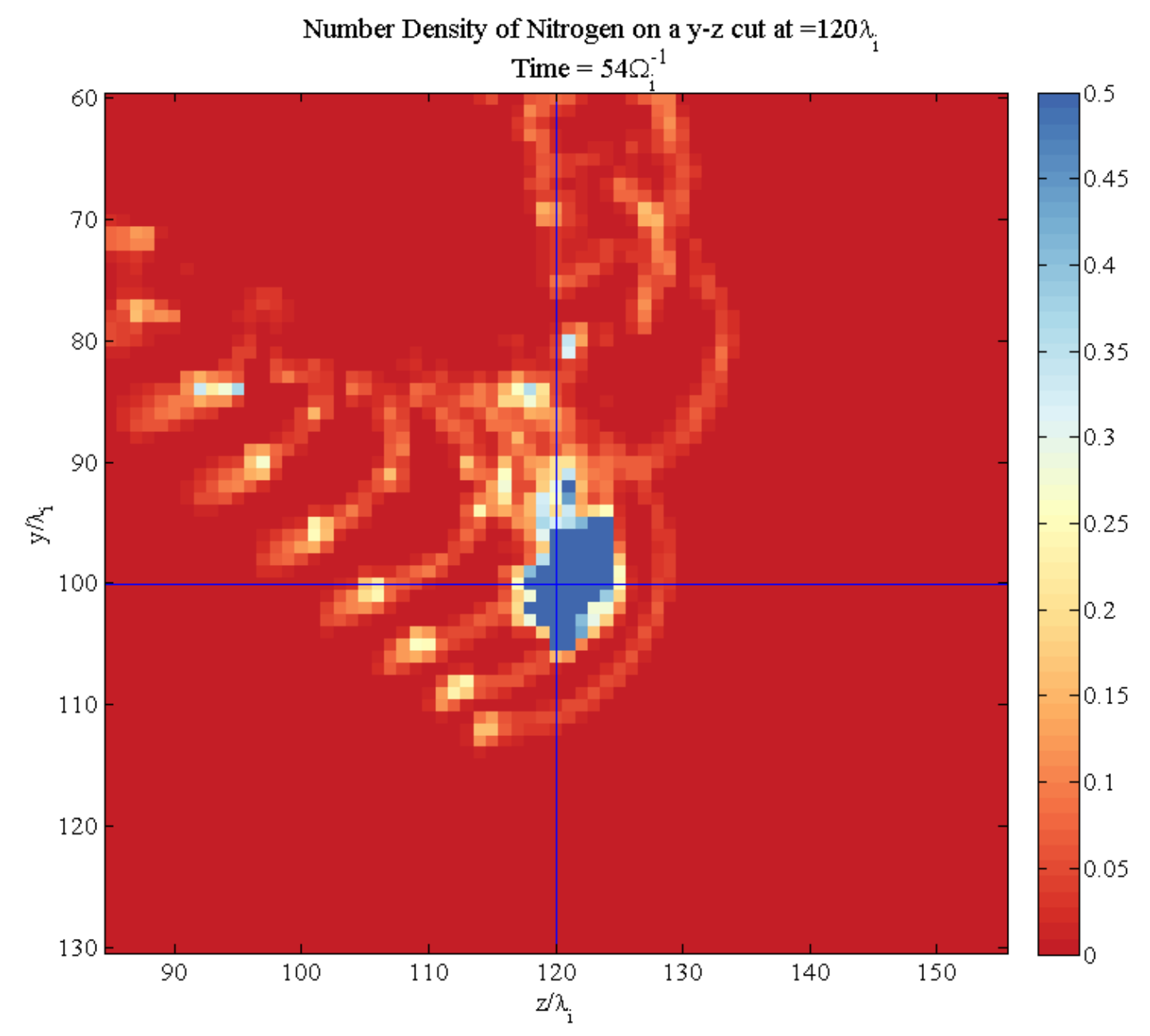

Figure 4.3.: A heatmap plot of the number density injected species on a $\hat{y}-\hat{z}$ plane through the centre of the simulation domain after $54 \Omega_{i}^{-1}$. This shows how the nitrogen is deflected in 'clumps' as it is deflected in the $-\hat{z}$ direction as it gyrates about the magnetic field lines.

Schematically, ion pickup is a result of neutral particles becoming ionised through some process, such as photo ionisation or impact ionisation, and having a difference in their velocity with respect to the solar wind. Due to this, the convective electric field acts to accelerate the newborn ions up to the solar wind velocity. Momentum conservation demands that the solar wind flow is slowed down by an equivalent 
amount in the local region to compensate for the acceleration of the picked up ions. The newborn ions then continue to gyrate about the magnetic field lines whilst being convected along with the solar wind flow ultimately having velocities between 0 and $2 V_{s w}$ as previously mentioned in Chapter 2 . However, the pickup process as seen in the simulations cannot be considered as 'textbook' given the significant asymmetries present, especially where the nitrogen component is concerned.

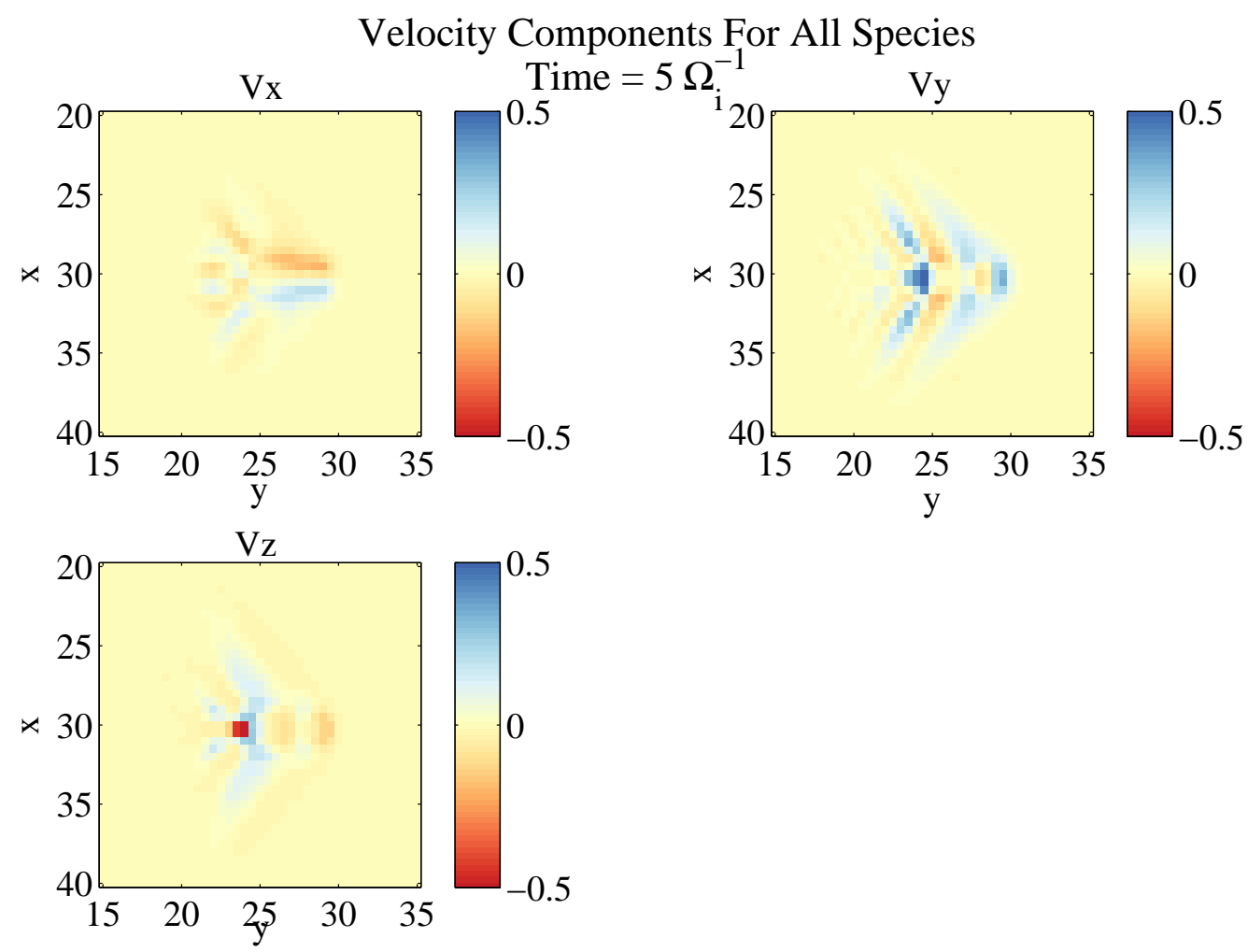

Figure 4.4.: The average velocity of the individual components of all particles in a cell after $5.0 \Omega_{i}^{-1}$. Axis units are in proton inertial lengths and the colour bar is relative to the Alfvén velocity. The disturbance to the solar wind velocity can be seen most clearly in the $\hat{x}$ and $\hat{y}$ components.

Figure 4.4 shows the average velocity taking into account all species (solar wind and thruster ions) at very early times in the simulation. From this state the effects only increase, since a structure can already be seen in the average velocity of the species it becomes clear that the mass loading effect will be significant once the interactions have developed. Since the simulation is performed in the frame of the solar wind, it will have zero ambient velocity initially. However, it can be seen from the $\hat{y}$ velocity component that the solar wind is being accelerated up to the injection velocity of the newborn ions in this frame which equates to a reduction of the solar wind flow speed in the solar frame. This effect is due to the mass loading that the 
thruster firing has caused via the ionisation of the neutral cloud. Additionally the flow can be seen to be polarised along $\hat{x}$ flowing away from the spacecraft

Figure 4.5 shows the combined number density of all species and that the solar wind is already disturbed by $7 \Omega_{i}^{-1}$ by the pickup of the newborn ions and perturbations in the density can be seen to be launched from the front of this induced interaction region which will later become a bow wave after sufficient mass has been loaded onto the solar wind flow to, in the simulation frame of reference, accelerate the ions to the pickup ion injection velocity.

Whilst this bow wave is initially weak, its amplitude grows over the duration of the simulation and propagates ahead of the spacecraft with a stand-off distance initially as small as $5 \lambda_{i}$ but increasing over time before fading away and being replaced by a newly formed wave behind. Although the injection of material is continuous, the injection rate is not constant. This is because the ionisation of the neutral cloud is an exponential process and so as the simulation progresses new ions are injected in slightly varying densities and over an ever increasing volume of the simulation domain. This leads to a lack of smoothness in the profile of the bow wave over time which can be seen in Figure 4.11 which shows the combined number density of all species for the first part of the simulation. Despite the relative smoothness of the injection the simulation never reaches a steady state. Whilst bow waves are regularly launched there is no truly steady flow of material ahead of the spacecraft, the system does reach a quasi-periodic state however in which there are periodic releases of clumps of material and the density of the core ion cloud increases a little more quickly.

Another result of this quasi-periodicity is that a Mach cone/Alfvén wings are formed behind the spacecraft [Drell et al., 1965, Neubauer, 1980] with a strength which is affected by the flow of material. As more ions are injected, the flow of the solar wind (or the lack thereof in the solar wind frame used for this simulation) is disturbed to a larger degree and the wings formed are both larger and more intense. Equally, when the injected ions have less relative motion (i.e. between the quasi-periodic events) then the wing structure generated begins to fade until this motion increases once again. This has the effect of forming sets of Alfvén wings which grow and fade in a similar manner to the wavefronts of the bow wave. These wings propagate away from the spacecraft with the expected phase speed of $1 V_{A}$. Additionally the magnetic fluctuations here are in anti-correlation with the fluctuations in the total number density.

In order to illustrate the bow waves, Figure 4.6 shows the magnitude of the magnetic field for $40 \Omega_{i}^{-1}$ along a line in the $\hat{x}-\hat{y}$ plane from $(\hat{x}, \hat{y}, \hat{z})$ co-ordinates 


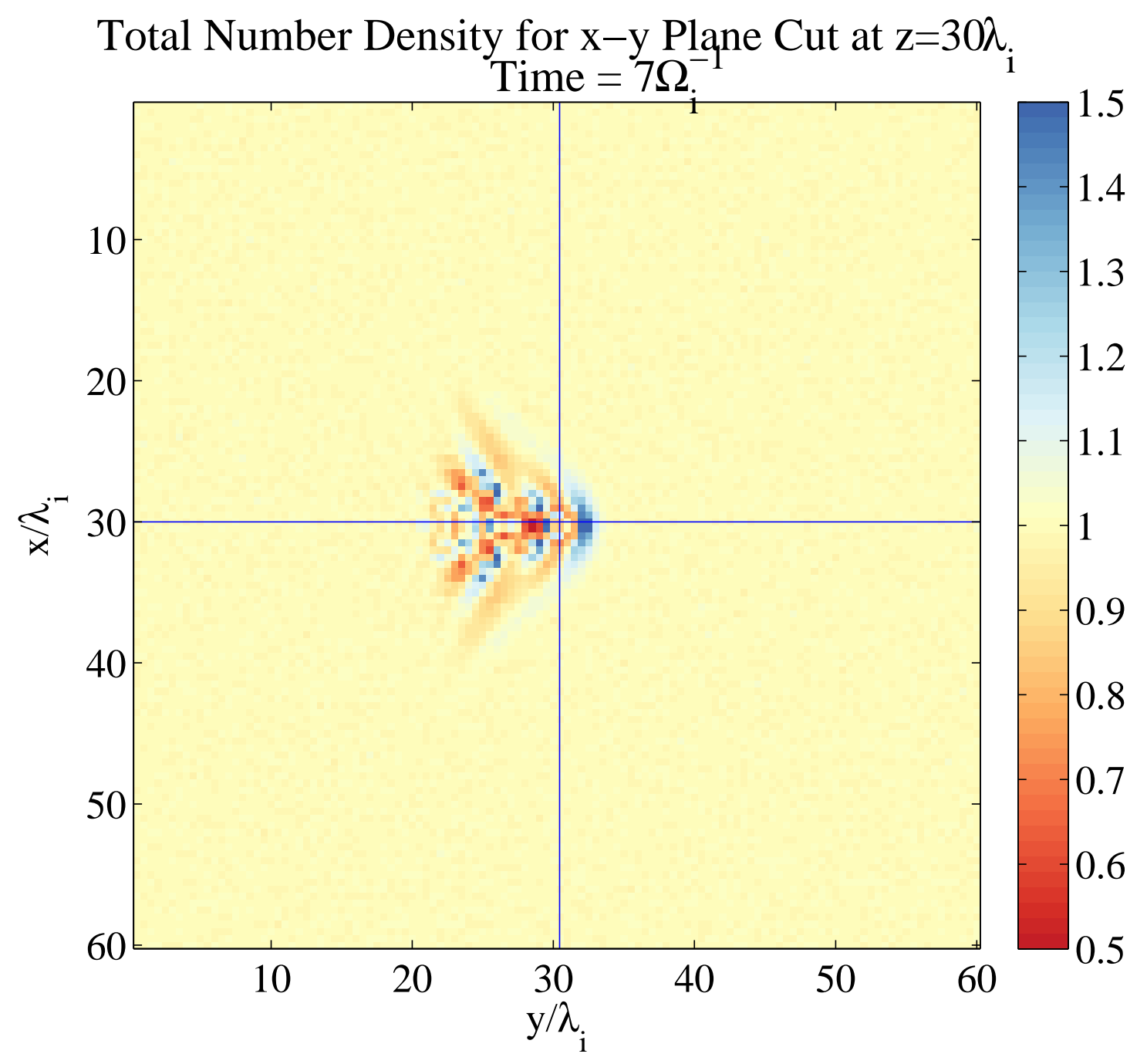

Figure 4.5.: The total number density of all species after $7.0 \Omega_{i}^{-1}$. Axis units are in proton inertial lengths and the colour bar is relative to the undisturbed solar wind number density. The formation of bow waves can be seen at the leading edge of the interaction region and already a periodicity to these waves is clear. The spacecraft marked by the crosshair is already enveloped by the interaction region. 


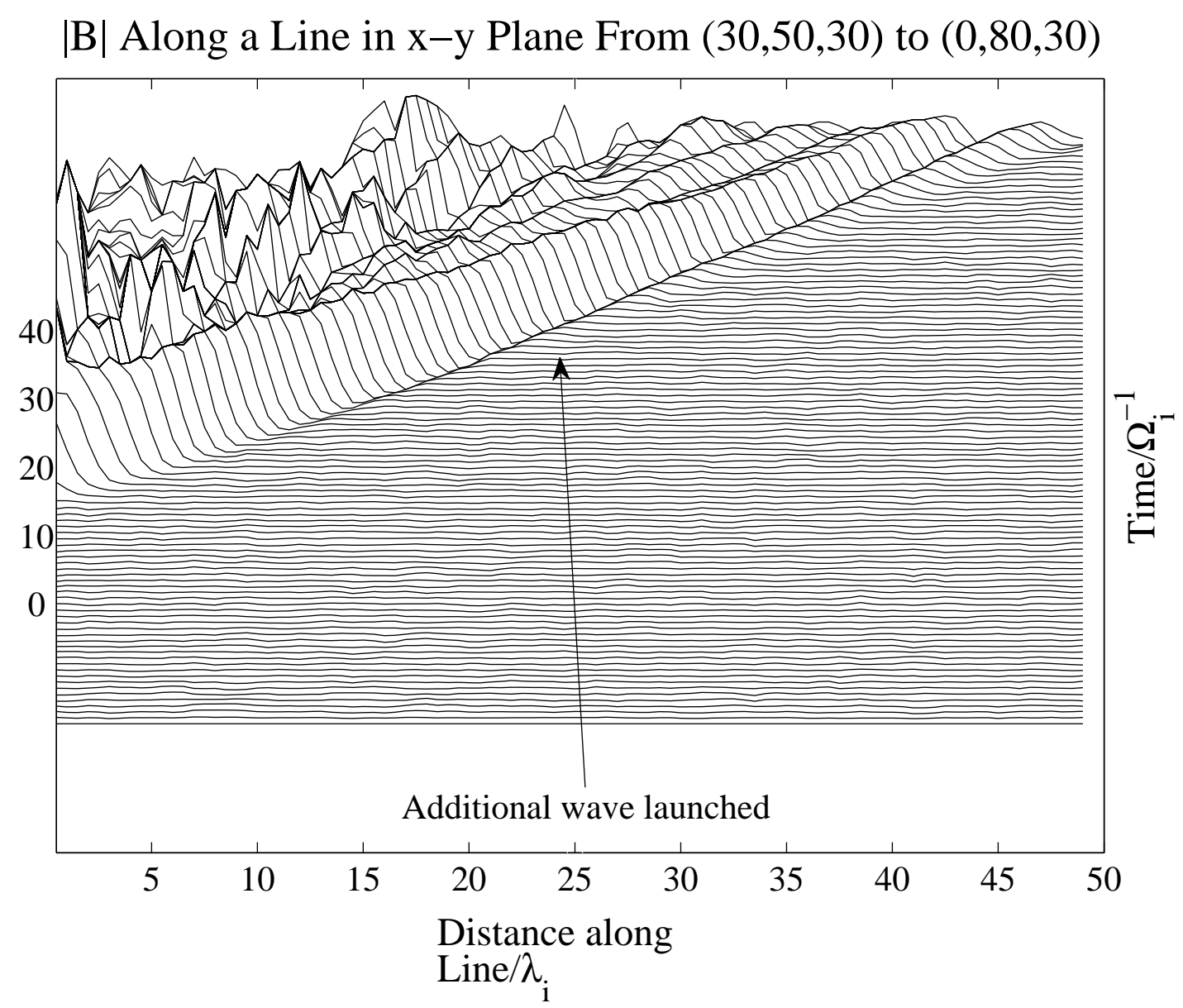

Figure 4.6.: A stacked line plot of $|B|$ from $0.0 \Omega_{i}^{-1}$ to $40.0 \Omega_{i}^{-1}$ showing the propagation of the main bow wave as well as the start of a wave being launched beyond the main bow wave. This can be seen by the relatively weak wave propagating away from the bow wave. The $\hat{x}$ axis shows distance along the strip measured in proton inertial lengths whilst the $\mathrm{y}$ axis shows the time in the simulation in cyclotron times. 
of $(30,50,30) \lambda_{i}$ to $(0,80,30) \lambda_{i}$. This line was chosen as the bow wave travels approximately in a straight line along it. The main wave can clearly be seen propagating along this line and after approximately $30.0 \Omega_{i}^{-1}$ an additional wave can be seen to be launched from the front of the main bow wave with a relatively small amplitude. Figure 4.6 also shows that towards the end of the simulation weak wave activity can be seen in front of the leading bow wave which means that the phase velocity of these waves is larger than the injection velocity of the newborn ions.

All of the mass being added to the solar wind flow and subsequently picked up by it also causes the ambient magnetic field lines to begin to drape around the induced interaction region formed before they begin to slip around the newly produced ion cloud. Figure 4.7 shows the magnetic field components for a planar cut through the simulation domain which indicates signs of field line draping: in the wings of the magnetic structure the magnitude of the field can be seen to fluctuate by approximately $25 \%$ of its undisturbed value and the perpendicular components of the field display the alternating field orientation associated with field line draping. The effects of draping, whilst not incredibly strong at these early times, are nonetheless of a significant strength and also extend large distances away from the spacecraft.

The magnitude of the magnetic field reflects to some extent the density enhancements in the solar wind. The magnetic field lines always curve along $+\hat{z}$ as they reach the interaction region before then curving in $-\hat{z}$ as they leave the interaction region, this is the structure that results in the hemispherical shells. At all planes of $\hat{z}$ the magnetic field drapes in the same way. The field never curves along $-\hat{z}$ first. The magnitude of curvature does change with the plane, however, as near the front of the interaction region the curvature is at its maximum and curves less towards the rear of the interaction region. Figure 4.8 shows the total number density of all species shortly after the simulation has begun. It can be clearly seen that the injected material is moving in the $+\hat{z}$ direction preferentially. This is due to the electric field induced at the front of the interaction region which accelerates particles along $+\hat{z}$ and thereby results in the preferential draping of the magnetic field lines along $+\hat{z}$.

Figure 4.9 shows the number density of the injected hydrogen component at $\mathrm{t}=120 \Omega_{i}^{-1}$. Over the long term the injected hydrogen ions are picked up in this sense and are left in a region behind the spacecraft gyrating about the magnetic field lines with a spread along the field lines that increases depending how far behind the spacecraft the region under consideration lies and hence how 'old' the ion is.

The nitrogen also displays a similar behaviour but its motion is on a much longer 


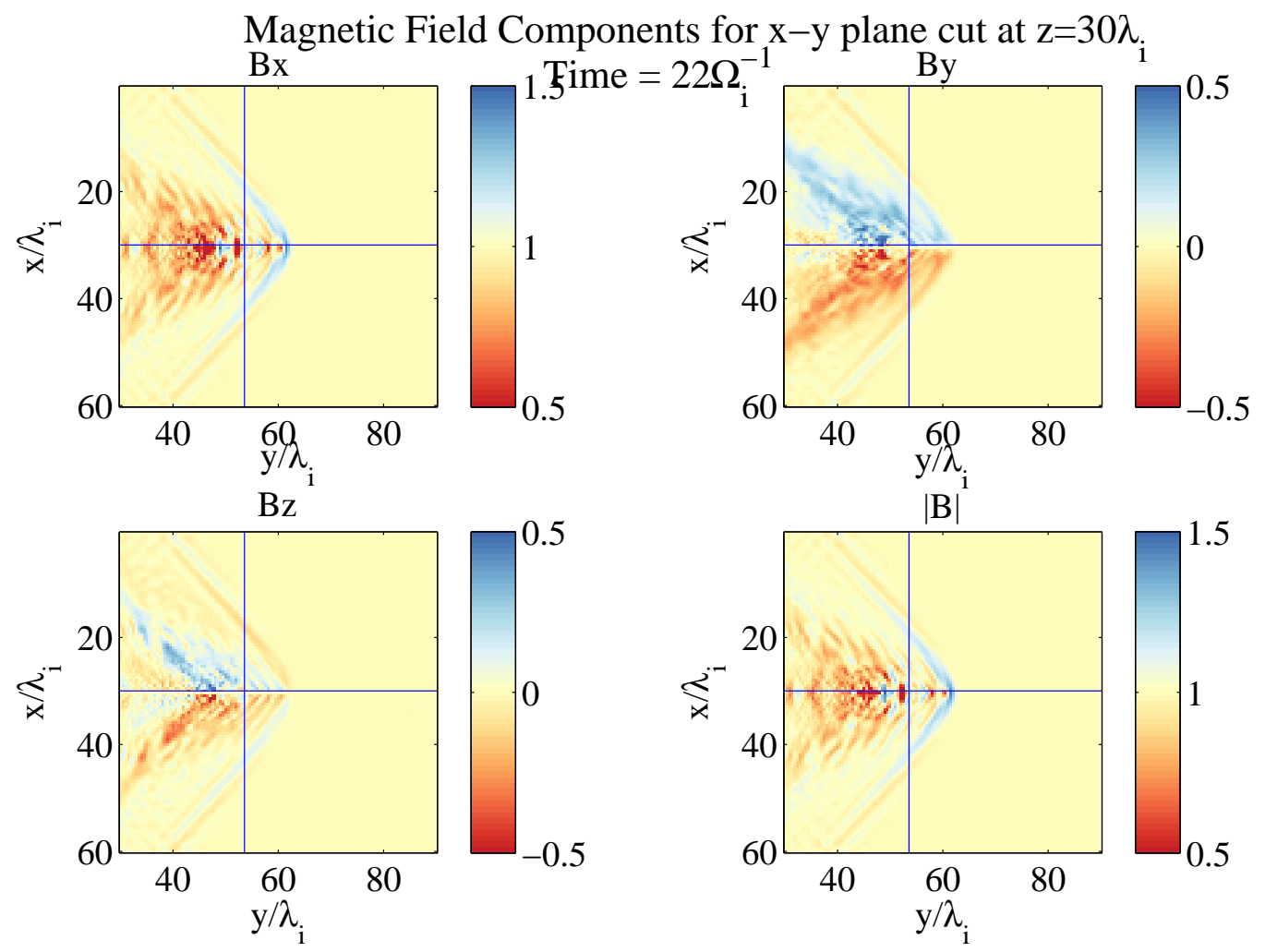

Figure 4.7.: The magnetic field components in the initial ambient field-spacecraft velocity plane $(\hat{x}-\hat{y})$ after $22.0 \Omega_{i}^{-1}$ showing a field line draping pattern. Axis units are in proton inertial lengths and the colour bar scale is relative to the undisturbed, ambient field strength. The crosshair shows the position of the spacecraft and the hydrogen component of the neutral cloud has the shape of a flattened sphere with a thickness of $2 \lambda_{i}$ in the ram direction and a width of $4 \lambda_{i}$ perpendicular to that and is centred $0.5 \lambda_{i}$ ahead of the spacecraft. 


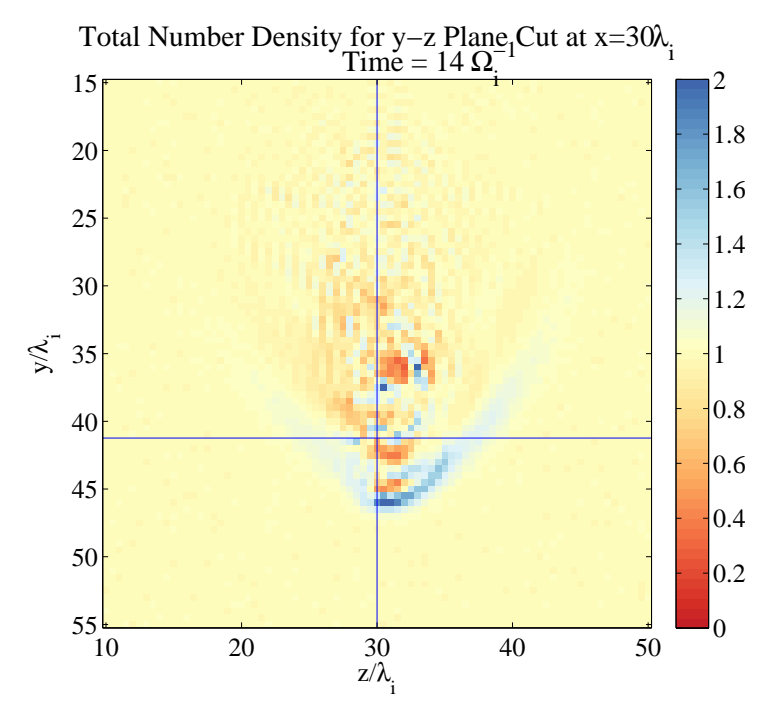

Figure 4.8.: The total number density of all species in the simulation after $14.0 \Omega_{i}^{-1}$. Axis units are in proton inertial lengths and the colour bar is relative to the undisturbed solar wind number density. An asymmetry along the $z$ axis can be seen ahead of the crosshair marking the spacecraft's position.

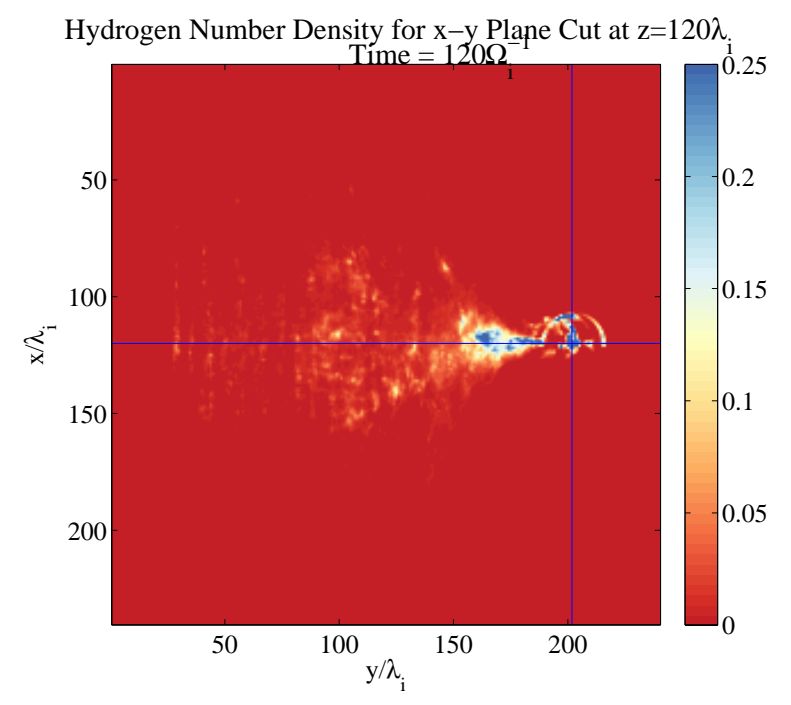

Figure 4.9.: The number density of just the hydrogen component of the injected material after $120.0 \Omega_{i}^{-1}$. Axis units are in proton inertial lengths and the colour bar is relative to the undisturbed solar wind number density. The ions have a much larger spread along the $\hat{x}$ axis at later times compared to the nitrogen component which remains confined largely to a region close to the spacecraft's trajectory. The spacecraft is marked by the crosshair and the hydrogen component of the neutral cloud has a thickness in the ram direction of $10.5 \lambda_{i}$ and a width perpendicular to that of $14 \lambda_{i}$. 


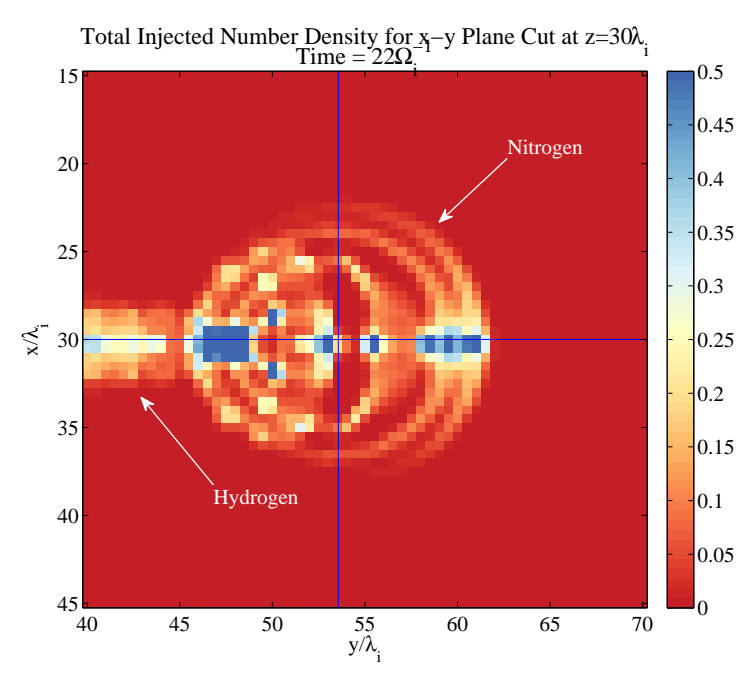

Figure 4.10.: The number density of only the injected material in the $\mathrm{x}-\mathrm{y}$ plane after $22.0 \Omega_{i}^{-1}$. Axis units are measured in proton inertial lengths. The colour bar scale is number density relative to the undisturbed solar wind number density. Distinct streams of nitrogen can be seen arcing in both the parallel and anti-parallel directions before rejoining a secondary cloud of material behind the spacecraft whose position is marked by the crosshair.

time scale (it requires longer than the total simulation duration to perform a single gyration) and is affected by the other drifts and disturbances it has been subjected to and thus is slightly more complex in nature. The nitrogen has typically not had sufficient time to become pitch angle scattered and is not as readily given a parallel velocity. The hydrogen eventually displays motion in the direction parallel to the initial, undisturbed ambient magnetic field once the strongest disturbances of the interaction region have passed by. As this is due to the more rapid pickup of the hydrogen component the nitrogen does not display this type of motion within the simulation duration but would be expected to with a simulation that both lasted a far greater time and had a much larger simulation domain. From Figure 4.10, which shows the number density of all injected species, it can be seen that the only parallel motion the nitrogen appears to perform is a semicircular gyration in the antiparallel direction before moving back into the line of its initial trajectory. At later times in the simulation the nitrogen has spread along the $\hat{x}$ axis and this can be attributed to the size of the neutral cloud exclusively.

The nitrogen has motion which is different to the hydrogen. The reason for the nitrogen component showing so little spread in the same way that the hydrogen does is due to the mass of the nitrogen being so much higher. Over an arbitrary duration the nitrogen ions will have completed less of a gyration than the hydrogen ions and 
so it will take longer for them to be scattered sufficiently along the parallel direction and to also have a fully gyrotropic velocity distribution.

Behind the spacecraft a dense clump of ions is formed over time. This large, dense region travels along the same trajectory as the spacecraft but with a slightly lower velocity. This large cloud of material is not deflected by the magnetic field as the front of the interaction region shields it from the effects by causing the magnetic field to drape around the interaction region which lowers the local magnetic field strength within the interaction region. This effect allows the ions to travel at slightly lower than the spacecraft's velocity without a significant drift away from that trajectory. This is similar to a 'tail' behind the spacecraft as it moves relative to the solar wind. The high density in the region results in fluctuations in the solar wind density which are slightly larger than those produced by the bow waves and fluctuations in the magnetic field which are significantly stronger than the bow waves.

As more and more material is injected into the solar wind and the mass loading becomes stronger an induced interaction region forms. This region is bounded at the front by the bow waves and the pile-up of the magnetic field and is bounded at the sides by the draped field lines. The rear of this induced interaction region is largely open and the hydrogen ions which become caught on the field lines can exit the region in this way. This strong interaction region has the main effect of containing the majority of the injected ions and their effects as pickup ions which encounter the front of this region are deflected such that they cannot easily pass through and, overall, has a lower density of solar wind protons. Within this region the ambient magnetic field has been reduced by the injection of ions such that there is a region approximately $10 \lambda_{i}$ across in which the field strength has been reduced to less than half its undisturbed value.

As the simulation progresses the size of the induced interaction region continues to increase as more mass is loaded onto ever increasing volumes of space such that the interaction region becomes quite large over the duration of the simulation although its relative effect does decrease slightly in some areas. As the newly injected material travels forward ahead of the spacecraft through a region of reduced ambient magnetic field it will encounter the front edge of the interaction region from the inside. The overall effect this has on the thruster ion flow is that it forms concentric, hemispherical shells of material along $\pm \hat{x}$ and in $\hat{z}$. These hemispherical shells appear as streams when a $2 \mathrm{D}$ planar cut is taken through the flowing material. These shells are launched from the front edge of the induced interaction region and travel over and behind the spacecraft where the flows all merge into a secondary ion cloud. The majority of this secondary ion cloud is composed of only nitrogen as it has a 
far larger gyroradius and also retains sufficient momentum to not be scattered as quickly as the hydrogen component which is, to a large extent, left behind in the hydrogen tail due to its far lesser mass.

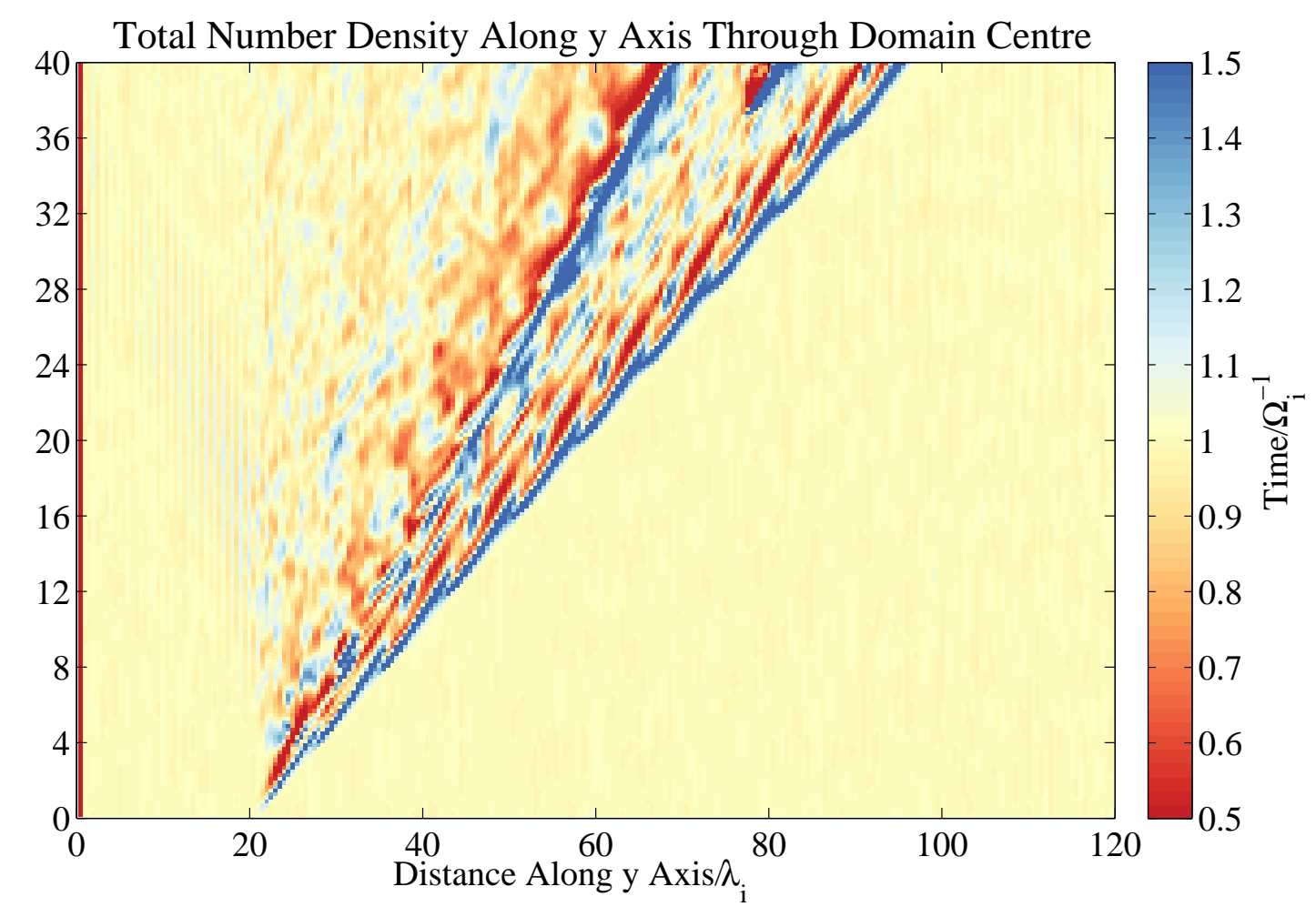

Figure 4.11.: A heatmap plot of the number density of all species from $0.0 \Omega_{i}^{-1}$ to $40.0 \Omega_{i}^{-1}$ showing the bow wave propagation along the $\hat{y}$ axis. The formation and the reformation of the main bow wave can be clearly seen as well as the motion of the secondary cloud that forms behind the spacecraft and follow along. The $\hat{x}$ axis shows distance along the strip measured in proton inertial lengths whilst the $\hat{y}$ axis shows the time in the simulation in cyclotron times.

Additionally the injection rate is not constant throughout the simulation as both the number of ions injected increases as does the size of the neutral cloud from which they are produced. For this reason the interaction region is not stationary over time and the bow waves formed are unsteady as they break and continually reform periodically. This can be seen in figure 4.11 which shows the number density of all species at early times as the leading bow wave is not smooth and steady but has a 'wavy' character as it breaks and reforms. The solar wind density can be significantly reduced in regions where the density of injected material is at its highest, at some places dropping as low as $25 \%$ of its undisturbed value. Additionally there remains some low level wave activity in the region behind the spacecraft when 
the solar wind density has recovered to near its ambient value. These fluctuations are at the $10 \%$ level and propagate predominantly along a line parallel to the $\hat{z}$ axis.

Shortly after injection the injected ions are not significantly scattered in pitch angle until an electric field is generated which then begins to scatter them slightly although they retain, to a large degree, their previous bulk velocity. The injected ions are 'picked up' or accelerated to the solar wind velocity by the action of the convective electric field. As the hybrid simulation is in the frame of the solar wind the electric field is produced by the relative motion of the injected ions and while in a solar frame the solar wind would slow down locally, in the hybrid simulation frame the solar wind will be accelerated to the ion injection speed.

$$
\begin{aligned}
\vec{E} & =-\vec{V}_{i n j} \times \vec{B} \\
\vec{E} & =V_{y} B_{x} \hat{z}
\end{aligned}
$$

Equation 4.1 shows that there will be an induced electric field in the solar wind frame at the forefront of the interaction region, where the ambient field and newborn ion velocity is as yet undisturbed, directed primarily along the $+\hat{z}$ direction with its strength being dependent on the injection velocity and the ambient magnetic field strength given the geometry used in the simulation. The ion injection velocity is the difference between the solar wind velocity and the spacecraft velocity and is most influenced by the solar wind bulk flow speed, although at small solar distances the spacecraft velocity becomes more significant. The injected material forms a relatively cold beam with a high energy compared to the solar wind flow and as the mass is loaded onto the magnetic field lines which are frozen into the solar wind flow this results in a slowdown of the local solar wind, in the solar frame, as the momentum is transferred to the injected ions as they are accelerated to the solar wind velocity. This can be seen by observing the magnetic field lines.

As the solar wind flow is accelerated, in the solar wind frame, in the region near the spacecraft the solar wind flow further away is initially unperturbed and continues at its ambient velocity and thus the magnetic field lines begin to pile up in front of the interaction region and form a magnetic barrier. As the magnetic flux is frozen to the solar wind the field lines are dragged along by the density pulse as it travels outward and hence the region of space where the thruster firing originated and the injected material remains is left with a deficiency of solar wind protons.

The pulse produced is similar to a bow wave in character as its peak amplitude 
lies in the ram direction with the intensity dropping off gradually as the angle of propagation with respect to the ram direction increases. The average phase velocity of this bow wave is approximately $1.9 V_{A}$, which is faster than the initial injection velocity of $1.5 V_{A}$. This can be seen in Figure 4.11 which shows the number density of all species for the first part of the simulation. In addition to this the effects of the expansion of the neutral cloud and a 'clumpiness' in the nitrogen component exerting an influence on the shape of the wave can be seen as the wave appears to slow down and break as it reforms slightly further ahead of where it would be expected to appear based on the wave being smooth and having a constant phase speed with a periodicity, holding constant throughout the simulation, of approximately $4.0 \Omega_{i}^{-1}$. Further, towards the end of the period captured in figure 4.11 the additional ion cloud, which forms behind the spacecraft's location, can be seen travelling a little slower than the main bow wave, such that it ends up being approximately $20 \lambda_{i}$ behind the main wave, with a similar periodicity in its enhancements but in contrast to the main bow wave this clump of ions does not disperse and travels at a constant speed throughout the simulation due to the shielding the leading bow wave provides it from the undisturbed solar wind.

The magnetic compression results in an increase in $B_{x}$ to approximately twice its undisturbed magnitude. This compression of the magnetic field also induces an electric field directed along $+\hat{z}$ and $-\hat{y}$ which acts to slow the injected material and deflect it from the thruster centreline. It is in this manner that the injected species are able to display the 'streaming' in the $\hat{y}-\hat{z}$ plane counter to their normally expected direction of gyration. Since

$$
-\frac{\partial \vec{B}}{\partial t}=\nabla \times \vec{E}
$$

If we take a point located along the trajectory of the spacecraft then the magnetic field will be compressed and hence the $B_{x}$ component will increase over time whilst $B_{y}$ and $B_{z}$ remain, ideally, at zero. Thus:

$$
\frac{\partial B_{x}}{\partial t} \hat{x}=\left(\frac{d E_{y}}{d z}-\frac{d E_{z}}{d y}\right) \hat{x}
$$

Which can produce an electric field directed along $-\hat{y}$ and $+\hat{z}$ where $E_{z}$ is largest and positive at the central point in question but decreases along $+\hat{y}$ whereas $E_{y}$ is smallest at the central point and decreases along $+\hat{z}$ but more slowly than the $E_{z}$ component which preserves the condition that the combination of the two remain positive. This also provides an electric field in the correct sense to deflect the 
positively charged ions along the $+\hat{z}$ axis before slowing them down so that the spacecraft passes them before the field reduces in magnitude which reverses the signs of the components of the electric field which causes the ions to move back to the thruster centreline where they then become a part of the secondary ion cloud.

The motion of the concentric shells of injected material along the $+\hat{z}$ direction may be due to a gradient in the magnetic field strength resulting from the pile up of flux because of the mass loading from the thruster firing. This gradient in $\vec{B}$ is encountered by the injected material which then experiences a drift according to:

$$
V_{\nabla B}=\frac{m v_{\perp}^{2}}{2 q B}(\vec{B} \times \nabla \vec{B}),
$$

where the drift velocity will be directed in the $\vec{B} \times \nabla \vec{B}$ direction, that is, along the $+\hat{z}$ axis. Additionally as the drift is proportional to the perpendicular energy of the ion experiencing the drift this results in the nitrogen having a higher drift speed than the hydrogen which is the reason for the shell being dominantly nitrogen in the $+\hat{z}$ direction and a combination of hydrogen and nitrogen in the $\pm \hat{x}$ direction. However, in order to confirm this the trajectories of individual ions would be a useful diagnostic and may shed light on some of the other mechanisms observed throughout the simulation such as what appears to be a periodic shedding instability at the forefront of the interaction region.

Additionally once the initial material has been injected and has begun to cause magnetic field line drag any subsequent material will be moving through a region with a lower local field strength and thus will have a velocity directed along $+\hat{y}$ relatively undisturbed until it reaches the front of the interaction region. This will then induce a convective electric field directed along $+\hat{z}$. The convective electric field combined with the induced electric field from the temporally varying magnetic field results in a reasonably strong acceleration of the newborn ions in the $+\hat{z}$ direction. The other component of the induced electric field acts to slow the flow of the ionised thruster material slightly so that in the solar wind frame it begins to travel more slowly than the spacecraft itself. As the material falls behind the spacecraft the $\hat{z}$ component of the electric field reverses and begins to accelerate the flow back towards the thruster centreline. Eventually this flow forms a cloud of significant density located behind the spacecraft with a velocity which is slightly slower than the injected velocity. This cloud continues to trail the spacecraft for the duration of the simulation whilst periodically ejecting clumps of material. Its density is also high enough that it produces some of the highest amplitude fluctuations seen in the simulation in both the density of the solar wind and the magnetic field. 

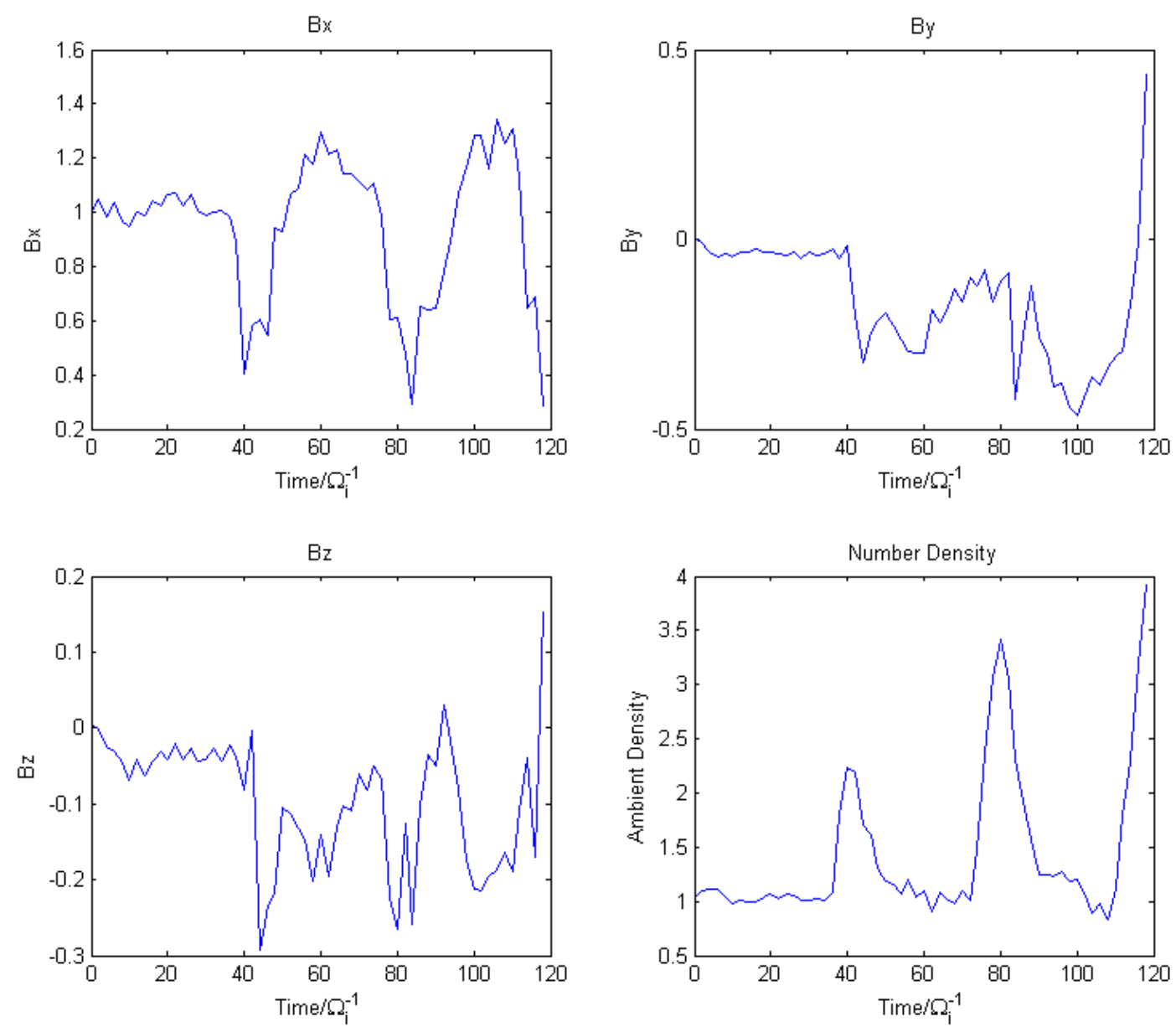

Figure 4.12.: A plot of the magnetic field and total density of ions that the spacecraft 'sees' throughout the duration of the simulation. There are several peaks in the density where the spacecraft encounters large regions of high density injected material along with drops in the ambient magnetic field as a result. 


\subsection{Conclusions}

Features which can be seen in this simulation include the pileup of magnetic field lines due to the mass loading of the solar wind. This pileup causes the field lines to drape about the interaction region that the injected cloud forms about itself and the field lines appear to slip around the interaction region preferentially in the $+\hat{z}$ direction and not at all in the $-\hat{z}$ direction. The parts of the interaction region with the densest concentration of injected ions can cause enough of a repulsive effect on the solar wind protons that the ambient solar wind density in those volumes of space can become significantly depleted for periods of time. The length of time the solar wind remains significantly disturbed appears to be similar to the simulation duration although even by the end of the simulation there are low level fluctuations present due to the injected ion gyration about the magnetic field lines of primarily the hydrogen component.

Additionally bow wave-like density pulses are produced which bound the interaction region and have the highest amplitude in the ram direction of several times the ambient solar wind density. These waves travel with a phase speed of $1.9 V_{A}$. Streams of mass of both injected species are also observed along both the parallel and antiparallel directions and also along the $+\hat{z}$ direction. This is thought to be the result of an induced electric field with possible contributions from a magnetic gradient drift effect. A Mach cone/Alfvén wing like structure is produced as the simulation progresses. The main area where the ions are injected does not retain a significant density over the long term, instead the majority of the ions are deflected around the spacecraft's position and rejoin in a secondary region behind the spacecraft where the injected ion density reaches its peak. This region of enhanced density follows behind the spacecraft with a slightly lower velocity and causes significant magnetic perturbations whilst persisting over the entire duration of the simulation and showing no signs of imminent dispersion. It should also be stated explicitly that the mass loading effects dominate over pick-up ion instabilities in this simulation.

These results may also have some applicability to weakly outgassing comets or asteroids [Blanco-Cano et al., 2003, Wang and Kivelson, 1996]. It should also be noted that the increasing injection rate bears similarities to a comet approaching the Sun as its mass flow rate, and hence the rate of production of ions which are then picked up by the solar wind, would increase inversely proportionate to its solar distance. For all practical purposes the interactions we have described will result in large periods of time where spacecraft data taking on Solar Probe will need to be, at least, reduced and more likely shut down entirely in order to protect the sensitive 
instruments as the lifetime of the neutral cloud is of the order of tens of minutes and so too, therefore, is the period of injection where the rate of ionisation is not negligible which means that the lifetime of the interaction region could last for an extended period of time. For the simulations performed the recovery time of the disturbed solar wind does not appear to be particularly lengthy as the initial region which is disturbed at the beginning of the simulation is relatively calm again by the end of the simulation although the ions that have been picked up do remain in the vicinity and continue to cause small fluctuations to the density and magnetic field throughout the entire duration of the simulation.

Figure 4.12 shows the levels of magnetic field fluctuations and density that the spacecraft might be subjected to over the duration of the simulation. From this it can be seen that several large density peaks are observed as regions of high density injected material encounter the spacecraft and with this comes a drop in the ambient magnetic field and perturbations on the 30-50\% level which could prove to be of consequence. The density fluctuations tend to settle down and return to near ambient levels far more rapidly than the magnetic perturbations which do not recover over the observed duration.

The largest issue is likely to be the fact that the disturbances have a very good chance of propagating ahead of the spacecraft and therefore will enter regions which are already disturbed as it travels onwards for some time after the thruster manoeuvre. These regions will continue to be disturbed for as long as the neutral cloud still has a density sufficient to maintain a modest injection rate, however, these issues are likely to be reduced if the direction of the thruster firing is not in the ram direction and a firing into the spacecraft's wake would produce the same strong interaction but the new thruster geometry would allow the spacecraft to outrun the worst of the effects and potentially reduce the 'downtime' required for a thrust. The effects observed would also be likely to change based on the angle of the solar wind flow compared to the spacecraft's velocity with some geometries causing the produced ion cloud to linger in the vicinity of the spacecraft for longer than others. 


\section{Interaction of Spacecraft Thrusters with the Solar Wind - A Parameter Survey}

The following chapter will discuss the results and features of the series of simulations performed as a part of a parameter survey. Parameters that were varied are the injection angle $(\theta)$, or spacecraft velocity vector (all injections are in the ram direction with respect to the spacecraft), with respect to the ambient magnetic field and the injection rate in the simulation which is related to the size of the thruster firing and determines the total amount of mass loaded onto the solar wind flow. The parameters were given previously in table 4.1 in Chapter 4 and are restated here. They include solar wind conditions which might be found at ten solar radii and approximately $30 \mathrm{~g}$ of ions injected over the simulation duration directed at $90^{\circ}$ to the ambient magnetic field. The domain size is a cube of sides $240 \lambda_{i}$ and has a duration of $120 \Omega_{i}^{-1}$, the newborn particles are injected with a velocity of $1.5 V_{A}$, the magnetic field strength is assumed to be 500nT and the solar wind number density to be $10^{9} \mathrm{~m}^{-3}$. First an overview of these results will be given followed by a more in-depth presentation.

\subsection{A Qualitative Overview}

This section will give a broad overview of the features seen in the simulations, such as magnetic field pile-up and field line draping, as well as the specific simulation set-ups used. The types of interactions seen will be briefly mentioned in a qualitative and more descriptive manner with the quantitative discussion to follow in later sections.

\subsubsection{Injection at $45^{\circ}$ to the Initial Magnetic Field}

This simulation has the same solar wind parameters as the 'standard' or 'reference' simulation in that it is performed at the same solar distance of ten solar radii. 
These parameters were given in table 4.1 in Chapter 4 and were briefly restated at the beginning of this chapter. The ion injection rate used for both hydrogen and nitrogen remains the same as the base thrust used is unchanged such that over the duration of the simulation approximately $30 \mathrm{~g}$ of ions is injected. The simulation domain is also identical measuring at $240 \lambda_{i}$ per axis and the simulation duration is also $120 \Omega_{i}^{-1}$. The magnetic field remains directed along the $+\hat{x}$ axis but the injection direction is changed to be angled at $45^{\circ}$ to the $\vec{B}$ field in the $\hat{x}-\hat{y}$ plane. This means that the bulk velocity of the ions as well as the spacecraft is changed to be directed in the $\hat{x}-\hat{y}$ plane rather than along the $\hat{y}$ axis only as the injection is still in the ram direction with respect to the spacecraft. The initial location of the spacecraft is changed such that it begins at $\left(20 \lambda_{i}, 20 \lambda_{i}, 120 \lambda_{i}\right)$ rather than at $\left(120 \lambda_{i}, 20 \lambda_{i}, 120 \lambda_{i}\right)$ in order to compensate for the new motion of the spacecraft. Over the duration of the simulation the same quantity of mass is added and therefore any difference in effects are due to the injection geometry only.

The most striking feature, looking at the magnetic field structure in the plane the spacecraft moves in, that is, the $\hat{x}-\hat{y}$ plane, is the asymmetric pileup of the field lines as Figure 5.1 shows. As is to be expected from the angle at which the ions are injected with respect to the ambient magnetic field the main pileup occurs in the direction perpendicular to the field and thus it can be seen that $|B|$ has its highest values in the $45^{\circ}$ between the spacecraft's velocity vector and the $+\hat{y}$ axis. The addition of a parallel component to the ion's velocity results in the interaction region having a larger perpendicular spread as the 'cavity' formed is both larger and 'deeper' than the reference simulation at $90^{\circ}$ to $\vec{B}$. Further, some waves can be seen propagating parallel to the $\vec{B}$ field ahead of the spacecraft's $\hat{x}$ co-ordinate.

In the $\hat{x}-\hat{z}$ plane a field draping pattern can be seen in the $B_{y}-B_{z}$ components as the field lines are separated by the parallel motion and compressed by the perpendicular motion. This gives rise to a draping pattern different to the one seen in the $90^{\circ}$ case. In the $\hat{y}-\hat{z}$ plane a similar structure to the $90^{\circ}$ case can be seen as a magnetic bow wave is present travelling along the $\hat{y}$ axis with a skew in the $-\hat{z}$ direction.

The view differs, however, when looking at the combined number densities of all the species present. There is initially very little disturbance to the solar wind plasma other than some modest wave activity/density pulses. As the simulation progresses and the injected mass builds, a bow wave begins to form in the $+\hat{x}$ direction and, to a lesser extent, in the $+\hat{y}$ direction. As the main ion cloud and the spacecraft move ahead the bow wave is strengthened. As more and more field lines are swept up, the plasma frozen to it is also swept up and around the edge of the interaction region. 
The component of the bow wave propagating perpendicular to the ambient magnetic field direction is able to move relatively freely into the solar wind as it propagates ahead of the injected ions along that direction and is able to disperse as the distance between the wavefront and the interaction region's core increases. The parallel propagating component, however, cannot create the same kind of distance as there is no force to restrict the motion of the ions in this direction and hence the intensity of the bow wave in this direction increases to much higher levels than in the perpendicular directions. The view in the $\hat{x}-\hat{z}$ plane from Figure 5.2 shows a similar picture as the bow waves start by being tightly contoured to the interaction region but then moving further away from it in the perpendicular $( \pm \hat{z})$ directions whilst remaining close to the interaction region in the parallel $(\hat{x})$ direction.

\subsubsection{Injection at $0^{\circ}$ to the Initial Magnetic Field}

This simulation shares parameters with both the $45^{\circ}$ simulation and the reference $90^{\circ}$ simulation as it is also performed at a solar distance of ten solar radii. Again the ion injection rate used for both the hydrogen and nitrogen is the same as the $90^{\circ}$ and $45^{\circ}$ simulations as the base thrust used is the same, approximately $30 \mathrm{~g}$ of ions is injected over the duration of the simulation. The magnetic field lines are directed along the $+\hat{x}$ axis, as is the direction of injection. The bulk velocity of the ions and spacecraft is therefore also directed along the $+\hat{x}$ axis. The simulation domain remains a cube of $240 \lambda_{i}$ per side and has a duration of $120 \Omega_{i}^{-1}$. The initial location of the spacecraft is at $\left(20 \lambda_{i}, 120 \lambda_{i}, 120 \lambda_{i}\right)$. Again, the total mass injected over the simulation is the same as for the $45^{\circ}$ and $90^{\circ}$ simulations and thus the geometry is the only significant change for this simulation.

Once more, looking at the magnetic field structure in the $\hat{x}-\hat{y}$ plane in Figure 5.3, the most obvious feature is a relative lack of field line pileup ahead of the spacecraft when compared with the previous simulations. There is a low level pileup at the edges of the interaction region with an Alfvén wing-like shape. The core of the interaction region is characterised by a very sharp drop in $|B|$. This region begins to resemble a diamagnetic cavity as the field strength is, overall, much reduced in the volume of the interaction region with respect to the ambient, undisturbed solar wind levels.

The $\hat{x}-\hat{z}$ plane shows a similar picture as there is a cavity but with far less spread in the $\hat{z}$ direction than in the $\hat{y}$ direction. Whilst the 'bow wave' seen here is still relatively weak, it is stronger in the $-\hat{z}$ direction and several wavefronts exist. The $\hat{y}-\hat{z}$ planes are disturbed by the passing of the ion cloud as the field lines are pushed 
outwards and a magnetosonic wave is launched, however, the field recovers very rapidly and the perturbations drop to almost negligible levels quickly.

Due to the injection being parallel to the magnetic field there is no significant mechanism by which the spacecraft can leave ions behind it and thus they continue to move with the spacecraft and their density builds. The core cloud does manage to achieve some minor spread in the perpendicular direction. Also because of the parallel injection geometry it becomes possible for injected ions to be backscattered and become deflected backwards in the $-\hat{x}$ direction. Similarly, it is possible for some of the leading ions to be further accelerated in the $+\hat{x}$ direction and move ahead of the main cloud by significant distances.

Further, enough ions are able to move ahead of the spacecraft in this manner such that they begin to affect the magnetic field structure in a more significant way. As these ions travel ahead they cause the field lines to separate and, to a lesser extent, the solar wind plasma to be moved out of the region as they move into it. This effect is strong enough that towards the end of the simulation the region directly ahead of the spacecraft begins to have a reduced field strength by a non-negligible amount. Behind the spacecraft there exists a thin region where backscattered ions have passed with both a reduced solar wind density and field strength. Very low level wave activity can also be seen propagating perpendicular to the ambient $\vec{B}$ field.

The $\hat{x}-\hat{z}$ plane shows a similar picture although the solar wind density does become elevated in the $-\hat{z}$ direction enough that it can be called a bow wave but the $+\hat{z}$ part of this wave is far weaker and does not extend as far. Looking at the $\hat{y}-\hat{z}$ plane, very little occurs with the levels of the solar wind number density. There is a brief enhancement as the spacecraft and ion cloud pass through a plane and a modest density pulse is launched but then quickly decays such that the solar wind returns to relatively undisturbed levels very rapidly after this 'ripple' is produced.

\subsubsection{Low Injection Rate at $90^{\circ}$ to the Initial Magnetic Field}

The injection rate of this simulation is reduced in comparison to the reference simulation such that over the simulation's total duration the average injection rate is only $20 \%$ of the reference. Over the simulation $\approx 6.5 \mathrm{~g}$ of mass is injected in total. Other parameters are kept the same for this simulation. The geometry of the simulation is the same in all respects to the $90^{\circ}$ reference simulation. The magnetic field remains directed along the $+\hat{x}$ axis and the spacecraft's velocity and hence the bulk 
velocity of the injected ions is once more directed along the $+\hat{y}$ axis. The simulation duration is $120 \Omega_{i}^{-1}$ and the domain forms a cube of sides $240 \lambda_{i}$. The simulation is performed with solar wind conditions expected at a solar distance of ten solar radii.

As would be expected from the lower mass injection rate the disturbances generated by the addition of the newborn ions have a lower amplitude than the reference simulations. Additionally, the disturbances which are produced take a longer period of time in order to reach visible levels and are also less extensive over space. The Alfvén wings produced are a lot thinner and more diffuse. Due to this the bow wave formed is very weak and scarcely even forms fully by the end of the simulation. The pileup of field lines ahead of the spacecraft is still evident but to a far lesser degree and in fact some of the strongest magnetic effects observed in this case are associated with draping rather than pileup in the parallel direction.

In an $\hat{x}-\hat{z}$ plane which the spacecraft and cloud pass through there is very little disturbance generated relative to the reference simulation. Low amplitude magnetic waves are generated in a circular pattern as the ion cloud passes through with an asymmetry in the $-\hat{z}$ direction. In the perpendicular direction the effects remain qualitatively the same. Magnetic pileup can still be observed as well as an asymmetry along the $\hat{z}$ axis and wave activity behind the spacecraft. All of these things are, however, affected by the lower rate of mass loading as the pileup is not as strong, an interaction region cannot be said to have formed until much later and, perhaps most importantly, the total size of the interaction region is much smaller. Any wave activity is also quickly quenched by the solar wind plasma once the ions have passed by.

Looking at the number density of the solar wind during this simulation it becomes clear that the mass injected is a significant factor when estimating the effects of a thruster firing at perpendicular injection. Initially the solar wind is hardly disturbed at all above background fluctuations and later in the simulation the disturbances which are 'large' for this simulation do not achieve particularly large amplitudes relative to the reference simulation. There is no evidence of a region in which the solar wind plasma becomes particularly depleted. The main effect seen is a weak 'ripple' in the solar wind number density which rapidly returns to ambient, undisturbed levels. Similarly in the $\hat{x}-\hat{z}$ plane the ion cloud passes through with very little effect and only very minor perturbations can be seen.

The $\hat{y}-\hat{z}$ (perpendicular) plane shows more of an impact due to the injection as the cloud accelerates the solar wind protons and forms a weak bow wave ahead of the spacecraft. Additional perturbations are visible predominantly in the $-\hat{z}$ direction. Within the much smaller interaction region there is a very small volume in which 
the solar wind plasma density briefly drops relatively low.

\subsubsection{High Injection Rate at $90^{\circ}$ to the Initial Magnetic Field}

The injection rate in this simulation is enhanced in comparison to the reference simulation such that over the total duration of the simulation the average rate of addition of mass is $400 \%$ that of the reference $90^{\circ}$ simulation. Over the duration of the simulation in excess of $120 \mathrm{~g}$ of material is injected. This simulation, like the low injection rate run, has many of the same parameters as the reference simulation. The simulation geometry is the same as the base simulation. The magnetic field is directed along the $+\hat{x}$ axis and the injection is directed along the $+\hat{y}$ axis. The simulation domain is a cube of sides $240 \lambda_{i}$ and, again, is simulated for $120 \Omega_{i}^{-1}$. The simulation is performed with solar wind conditions expected at a solar distance of ten solar radii.

Due to the much higher injection rate in this simulation an interaction region is formed almost immediately and the magnetic field is very rapidly swept up ahead of the spacecraft into a bow wave. These effects are very strong in comparison to the previous simulations. The Alfvén wings produced as well as the draping effects are not only strong but are also far more extensive. Bow waves are stimulated and formed so rapidly that they do not have sufficient time in which to propagate away from the spacecraft and subsequent waves merge with previous ones as they catch up due to having a higher phase speed. Additionally the interaction region becomes very turbulent due to the current produced by the motion of the injected ions. In an $\hat{x}-\hat{z}$ plane which the cloud passes through the effects can also be seen strongly as multiple wavefronts are quickly launched in concentric, circular patterns with strong field draping patterns in the perpendicular components of the magnetic field.

The view is similar when looking at the perpendicular $\hat{y}-\hat{z}$ plane as Figure 5.5 indicates. The field can be seen to pile up very quickly as $B_{x}$ shows rapid, high amplitude and extensive enhancements. The perpendicular components in this plane show a surprising lack of strength in the perturbations when compared with those present in $B_{x}$ and instead it appears that the inner interaction region is, in a sense, diamagnetic in that the field associated with the solar wind plasma is largely excluded and the main source of magnetic fluctuations is the injected ion motion itself. The interaction region formed in this simulation is also far larger than the one formed during previous simulations.

As with the magnetic field, the solar wind number density is heavily affected by 
this injection as multiple bow waves are formed and are linked at a point directly ahead of the spacecraft. These waves are high in amplitude and are, therefore, slow to disperse. Whilst the solar wind density does recover, it takes a longer time than in other simulations. Also unlike other simulations the inner interaction region has ejected the majority of the solar wind plasma such that its density there drops very close to zero in some places and is significantly depleted elsewhere. This picture is repeated in the $\hat{x}-\hat{z}$ plane as the core of the cloud passes through and produces ripples in the solar wind and completely pushes the solar wind plasma out of the core region as the plasma and magnetic field lines are swept up.

In the $\hat{y}-\hat{z}$ plane it can be seen that the interaction region is not as extensive along the $\hat{z}$ axis. The solar wind achieves significant densities ahead of the spacecraft, swept by the cloud core, and the region behind this is left depleted of solar wind protons. There also exists an asymmetry in the $-\hat{z}$ direction as the solar wind is more depleted towards $-\hat{z}$ than $+\hat{z}$. The recovered solar wind has larger background fluctuations in density than the undisturbed solar wind.

\subsection{Magnetic Pileup}

\subsubsection{Injection at $45^{\circ}$ to the Initial Magnetic Field}

For the simulation with the injection at $45^{\circ}$ to the ambient magnetic field Figure 5.1 shows that the pileup of the magnetic field lines is reasonably strong but highly asymmetric in the $\hat{x}-\hat{y}$ plane with respect to the spacecraft's trajectory. As the injection velocity has a parallel component the pileup is significantly reduced in terms of angular coverage (see Figure 4.7). Where for the $90^{\circ}$ simulation the pileup is surrounding the entire spacecraft, in this $45^{\circ}$ simulation the pileup initially only extends in the perpendicular directions. This then extends further which shows as a pileup in the strength of the perpendicular magnetic field components along the $+\hat{x}$ direction as the field begins to drape around the interaction region.

Figure 5.2 shows that in the $\hat{x}-\hat{z}$ plane the enhancements to the magnetic field are more symmetric with respect to the spacecraft's ram direction. The $B_{x}$ waves/pileup region is shaped much like the $90^{\circ}$ case but with a 'gap' at very small angles to the ambient field direction. At greater angles than $\approx 10-15^{\circ}$ the field lines can be seen to accumulate due to the motion of the injected ions as they cause loading onto the solar wind and cause the field lines to be dragged along with them in the $\hat{x}-\hat{y}$ plane but could be more accurately described as parting the field lines when looking at the $\hat{x}-\hat{z}$ plane. The $\hat{y}-\hat{z}$ plane shows the pileup to be completely symmetric on either 

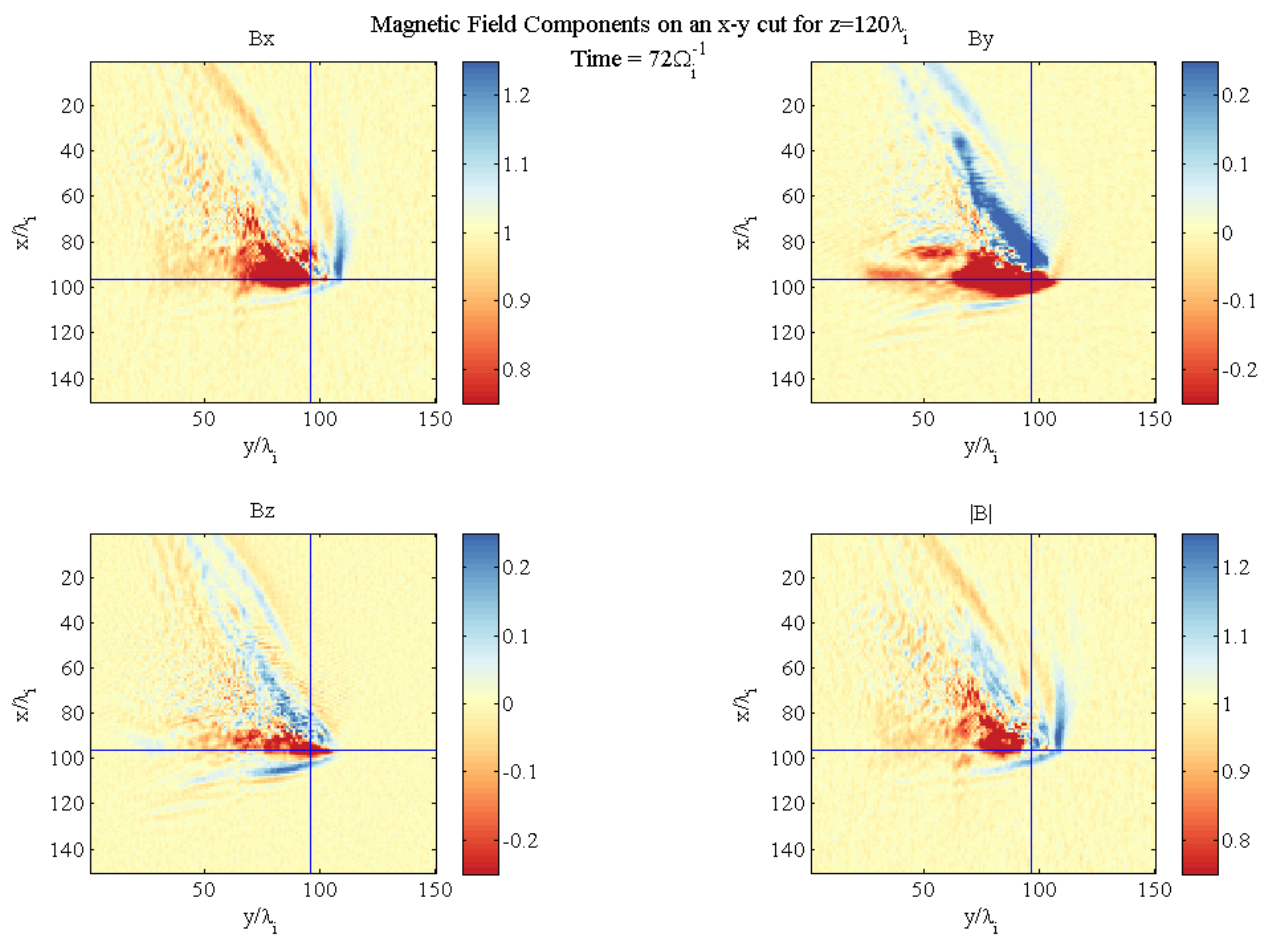

Figure 5.1.: The magnetic field on an $\hat{x}-\hat{y}$ plane at $\hat{z}=120 \lambda_{i}$ in the $45^{\circ}$ simulation domain after $72 \Omega_{i}^{-1}$. The flux pileup can be seen to be asymmetric with respect to the spacecraft's ram direction. 

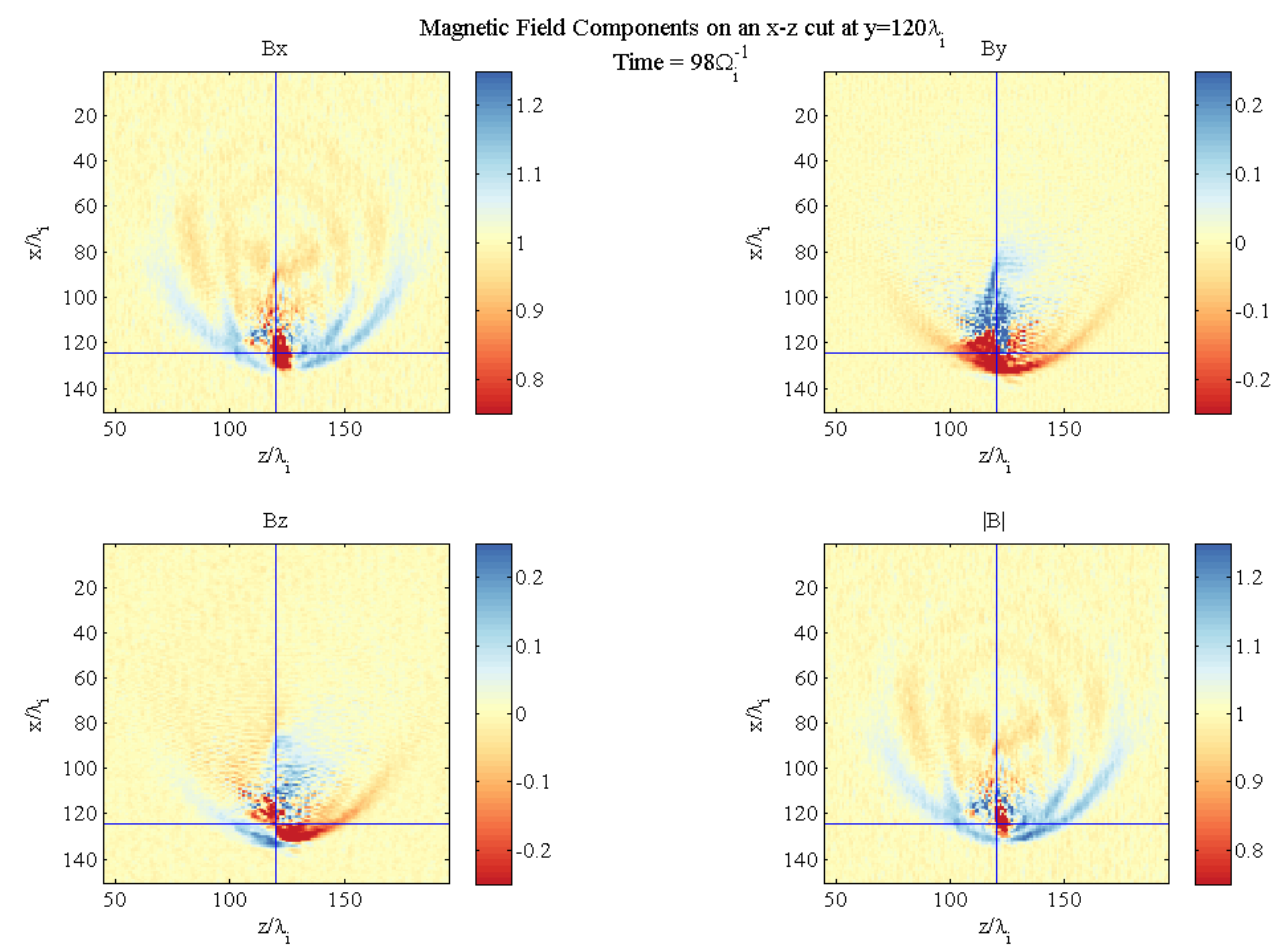

Figure 5.2.: The magnetic field on an $\hat{x}-\hat{z}$ plane at $\hat{y}=120 \lambda_{i}$ in the $45^{\circ}$ simulation domain after $98 \Omega_{i}^{-1}$. The pileup in this case is relatively symmetric along the $\hat{z}$ axis with a small break in the pileup in the centre, i.e. the spacecraft's ram direction in this plane.

side of the spacecraft and launching multiple waves in much the same manner as the $90^{\circ}$ case.

\subsubsection{Injection at $0^{\circ}$ to the Initial Magnetic Field}

The simulation at $0^{\circ}$, that is, where the injection is parallel to the magnetic field lines shows a different picture once again. In the $\hat{x}-\hat{y}$ plane there is initially no pileup of field lines whatsoever as the injected ions merely travel along the field lines without greatly disturbing them. However, for much the same reason this means that the ions will continue to travel with the spacecraft and subsequent injections will serve to increase the density of its core. After this density has risen enough, the expansion of the cloud and mutual repulsion of the ions will play a more prominent role as ions are 'squeezed' out of the cloud. This has the effect of causing the field lines to separate and move apart ahead of the spacecraft.

In the early stages of this simulation there is almost no pileup, it would be a better description to say that there are localised enhancements in $B_{x}$ due to field 

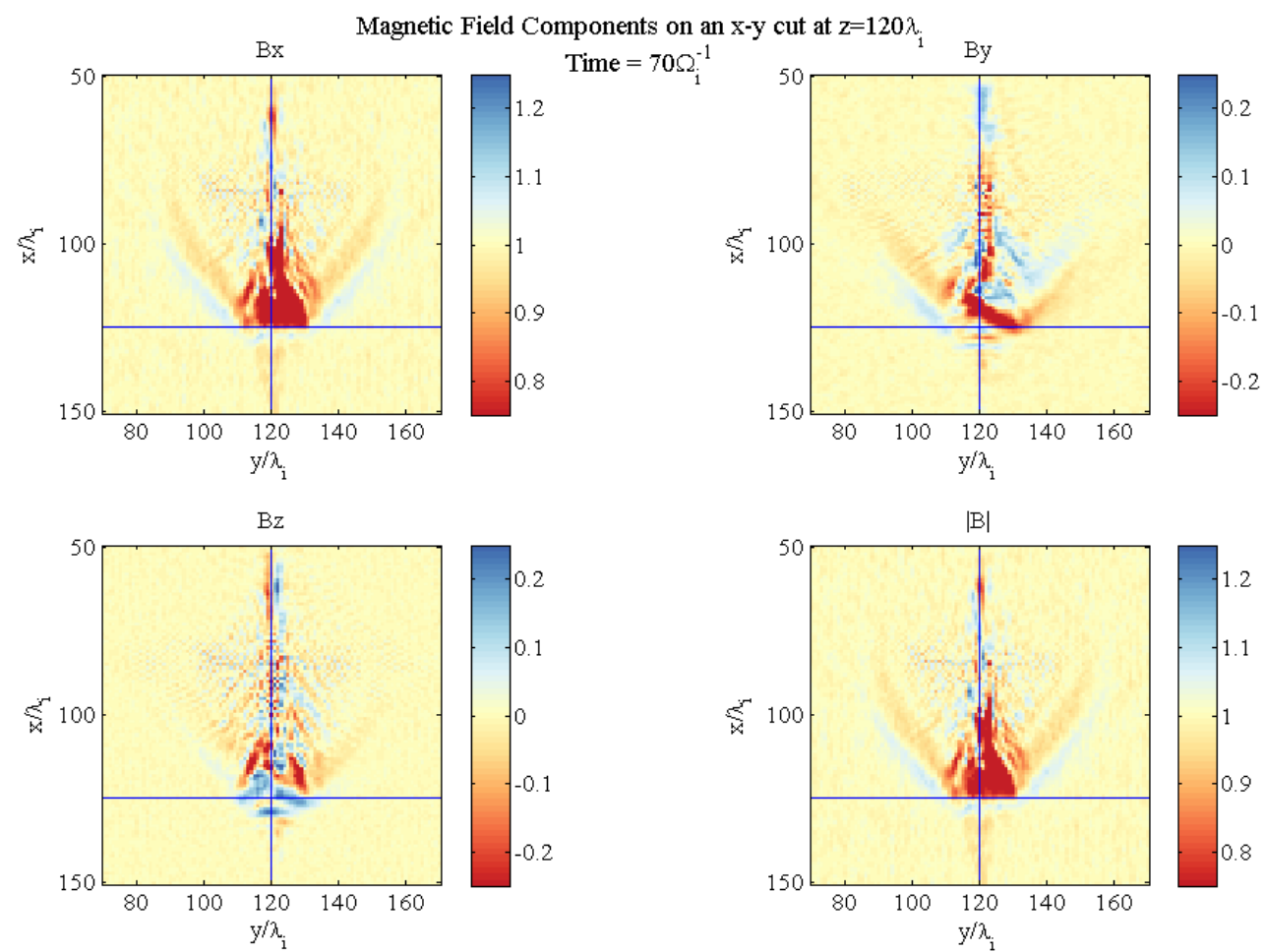

Figure 5.3.: The magnetic field on an $\hat{x}-\hat{y}$ plane at $\hat{z}=120 \lambda_{i}$ in the $0^{\circ}$ simulation domain after $70 \Omega_{i}^{-1}$. The magnetic pileup of flux in this simulation is relatively minor in comparison to others. Only low levels of pileup are seen ahead of the spacecraft. 
lines 'rippling' from the disturbance of the ion cloud's formation and expansion rather than a 'true' pileup which would have a more stable structure with respect to the spacecraft and ion cloud core. After $60 \Omega_{i}^{-1}$ the core has accumulated sufficient size and density to begin to cause the field lines to curve more significantly around it and this is shown in Figure 5.3 as there is no significant pileup ahead of the spacecraft but flux is gathering along the wings as the field lines are separated. At this stage some weak pileup occurs at the edges of the cloud akin to streamlines. The 'wings' are at approximately the $5 \%$ level and are due to the cloud parting the field lines nearby and forcing them to travel around the edge of it. The pileup strengthens over the duration of the simulation to a maximum of $10 \%$ level fluctuations but remains weaker at all times to comparable situations for other injection angles.

In the perpendicular directions (the $\hat{y}$ - $\hat{z}$ plane) the region of pileup is circular but weak and travels and disperses rapidly, as expected. The $\hat{x}-\hat{z}$ plane pileup is very similar to that in the $\hat{x}-\hat{y}$ plane but with a slight asymmetry in the $\hat{z}$ direction due to the gyration of the newborn ions as seen in Figure 5.8 in section 5.3.2. The enhancements are moderately stronger in the $-\hat{z}$ direction than in $+\hat{z}$.

\subsubsection{Low Injection Rate at $90^{\circ}$ to the Initial Magnetic Field}

The low injection rate simulation shares many features with the reference simulation. The main differences are in the levels of fluctuations seen. The pileup of field lines is much slower to occur throughout this simulation and where levels reach $30-40 \%$ for the reference simulation, in this case it only reaches $15 \%$ levels. Further, it takes a longer period of time for the disturbances and pileup to reach observable levels taking on the order of $20-30 \Omega_{i}^{-1}$ rather than the $5-6 \Omega_{i}^{-1}$ in the reference simulation. For the majority of the simulation the pileup remains at very mild levels, it starts to reach competitive levels of around $10 \%$ after approximately $100 \Omega_{i}^{-1}$, after a far longer period than the reference simulation.

The extent of the magnetic pileup is much reduced also as the region which is disturbed is reduced from $\approx 70 \lambda_{i}$ in the reference $90^{\circ}$ simulation to a mere $20 \lambda_{i}$ in either direction as the parts of the pileup region which extend further have reduced in amplitude far enough that the fluctuations are only comparable to the background noise. In the $\hat{y}-\hat{z}$ plane the interaction remains qualitatively the same as the reference but again with reduced levels and less peaks in the magnetic field strength. Where the reference has four peaks and a maximum of over $30 \%$, the low injection rate simulation only has two peaks with a maximum of $18 \%$ level fluctuations as shown 

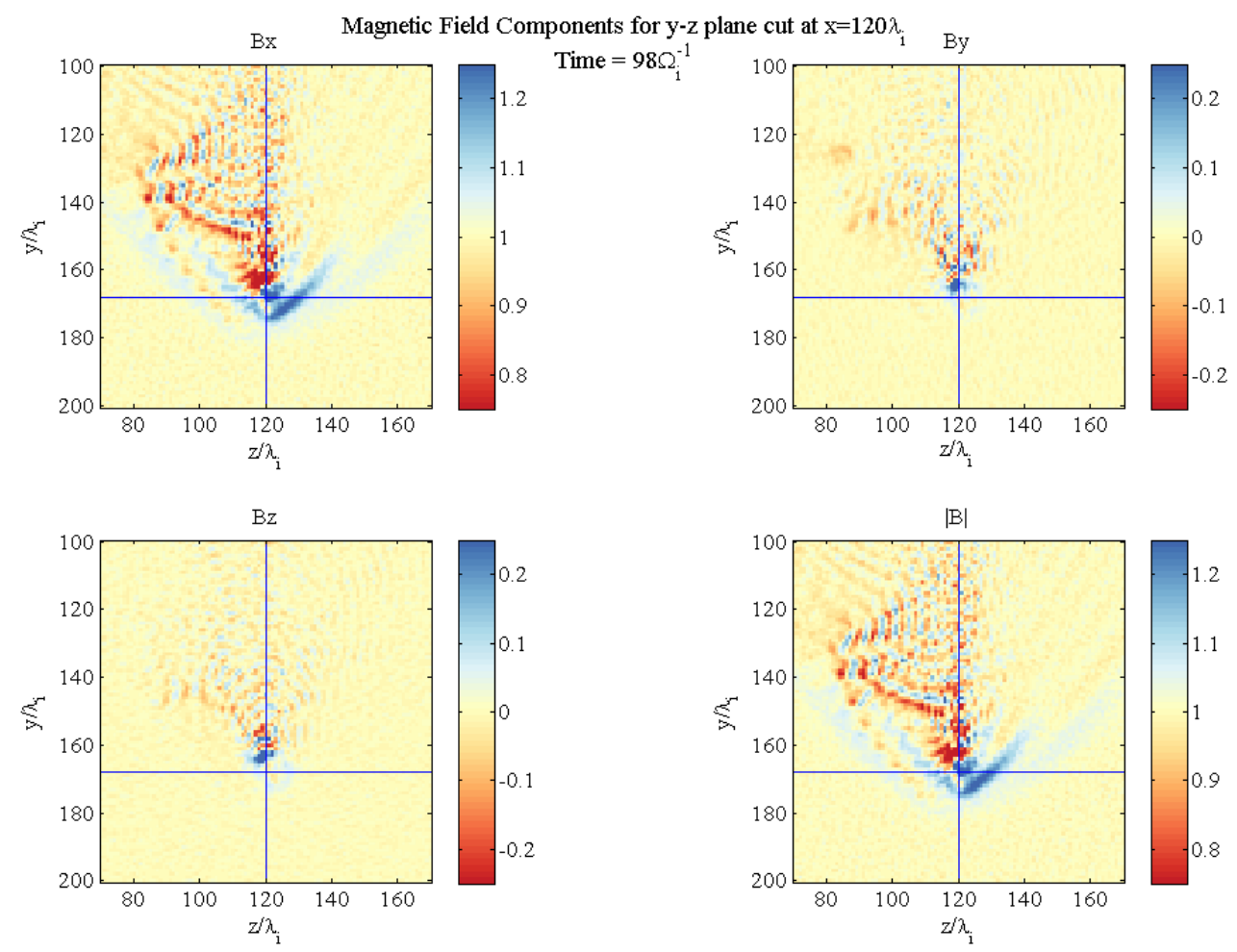

Figure 5.4.: The magnetic field on an $\hat{y}-\hat{z}$ plane at $\hat{x}=120 \lambda_{i}$ in the low injection rate simulation domain after $98 \Omega_{i}^{-1}$. Modest pileup of flux can be seen ahead of the spacecraft primarily in the $+\hat{z}$ direction. Peak values reach the $20 \%$ level. Injection angle is at $90^{\circ}$ to the initial magnetic field direction. 
in Figure 5.4.

\subsubsection{High Injection Rate at $90^{\circ}$ to the Initial Magnetic Field}

In the high injection rate simulation the magnetic pileup begins immediately and very quickly reaches levels of approximately $50 \%$ and higher. The injection rate in this run is so high that the waves in the pileup regions do not get the chance to form individually and instead form on top of each other. Figure 5.5 shows the extent of the pileup even at relatively early times as the leading waves are merged and multiple wave fronts can be seen. These merged waves do not begin to resolve into individual pulses until further than $80^{\circ}$ from the spacecraft's velocity vector from the point of view of the spacecraft. The thickness of the pileup region in the ram direction is $\approx 10 \lambda_{i}$. Further, the extent of the pickup region extends a lot further than in previous simulations. The $\hat{y}-\hat{z}$ plane shows, once again, a similar structure in the shape of the pileup region with very high level fluctuations throughout and also with additional waves produced in the $+\hat{z}$ direction. This is due to expansion effects of the neutral cloud, and hence, the injected ions.

\subsection{Magnetic Field Draping}

Each of the simulations performed for this survey exhibit signs of field line draping. The precise characteristics of this are modified by the different effects of the changing injection geometry and mass loading rate from the different thruster burn sizes and durations.

\subsubsection{Injection at $45^{\circ}$ to the Initial Magnetic Field}

Once again the run with injection at $45^{\circ}$ to the magnetic field shows an immediate asymmetry between the parallel and perpendicular directions in the $\hat{x}-\hat{y}$ plane. As the injection direction is in the $\hat{x}-\hat{y}$ plane for this simulation the field draping is skewed similarly. As expected, the major component of the draping is in the $B_{y}$ component of the magnetic field as the field lines are dragged in the $+\hat{y}$ direction along with the motion of the ion cloud such that the enhancements are to $+B_{y}$ behind the spacecraft's position at almost $45^{\circ}$ as the field lines are curved from behind the spacecraft to pass in front of the spacecraft and then continue onwards along $+\hat{x}$ and this is shown in Figure 5.1. As a result of this, once past the spacecraft, 

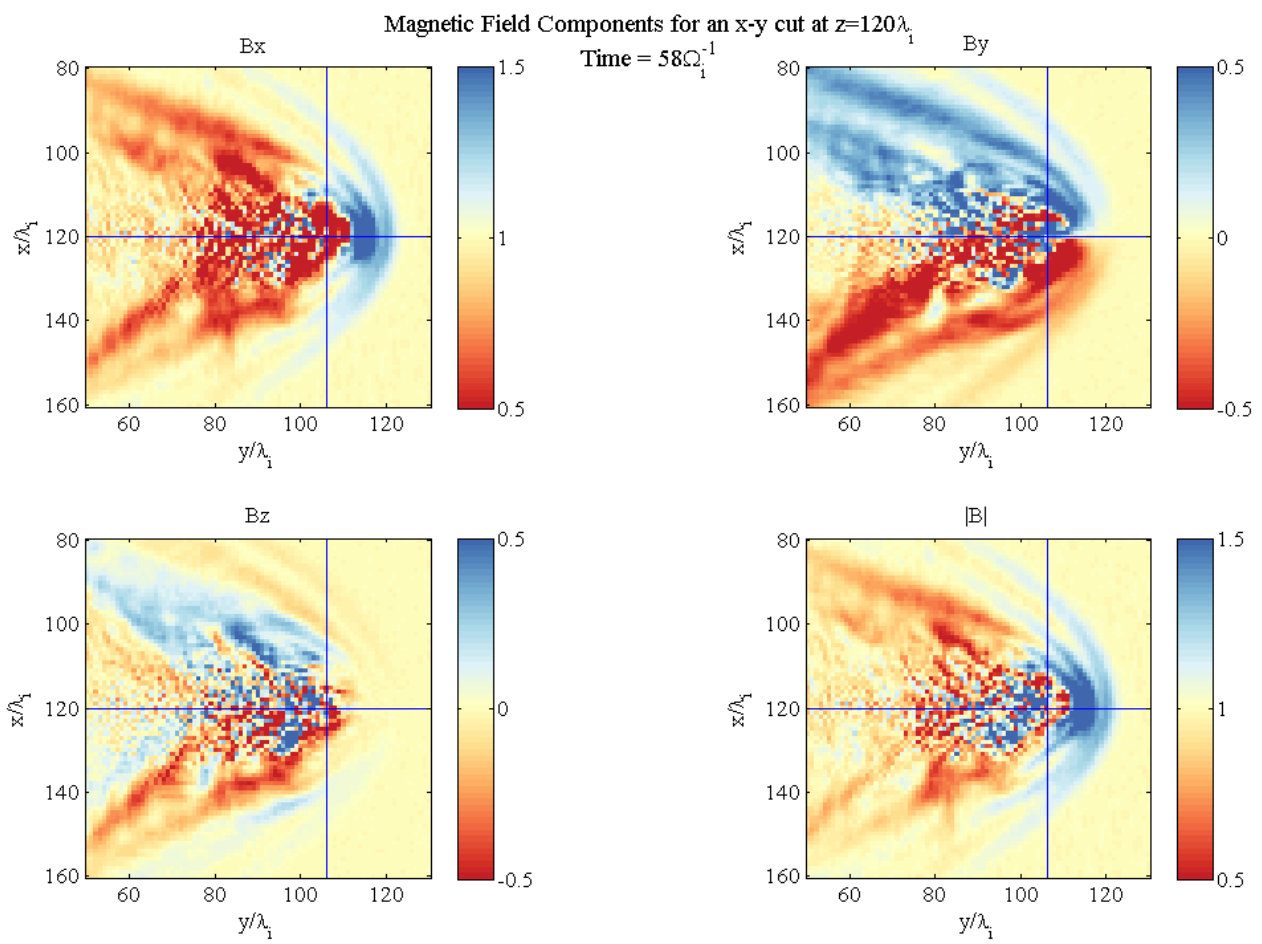

Figure 5.5.: The magnetic field on an $\hat{x}-\hat{y}$ plane at $\hat{z}=120 \lambda_{i}$ in the high injection rate simulation domain after $58 \Omega_{i}^{-1}$. Large amplitude and extensive flux pileup is observed with multiple wavefronts. Injection angle is at $90^{\circ}$ to the initial magnetic field direction. 

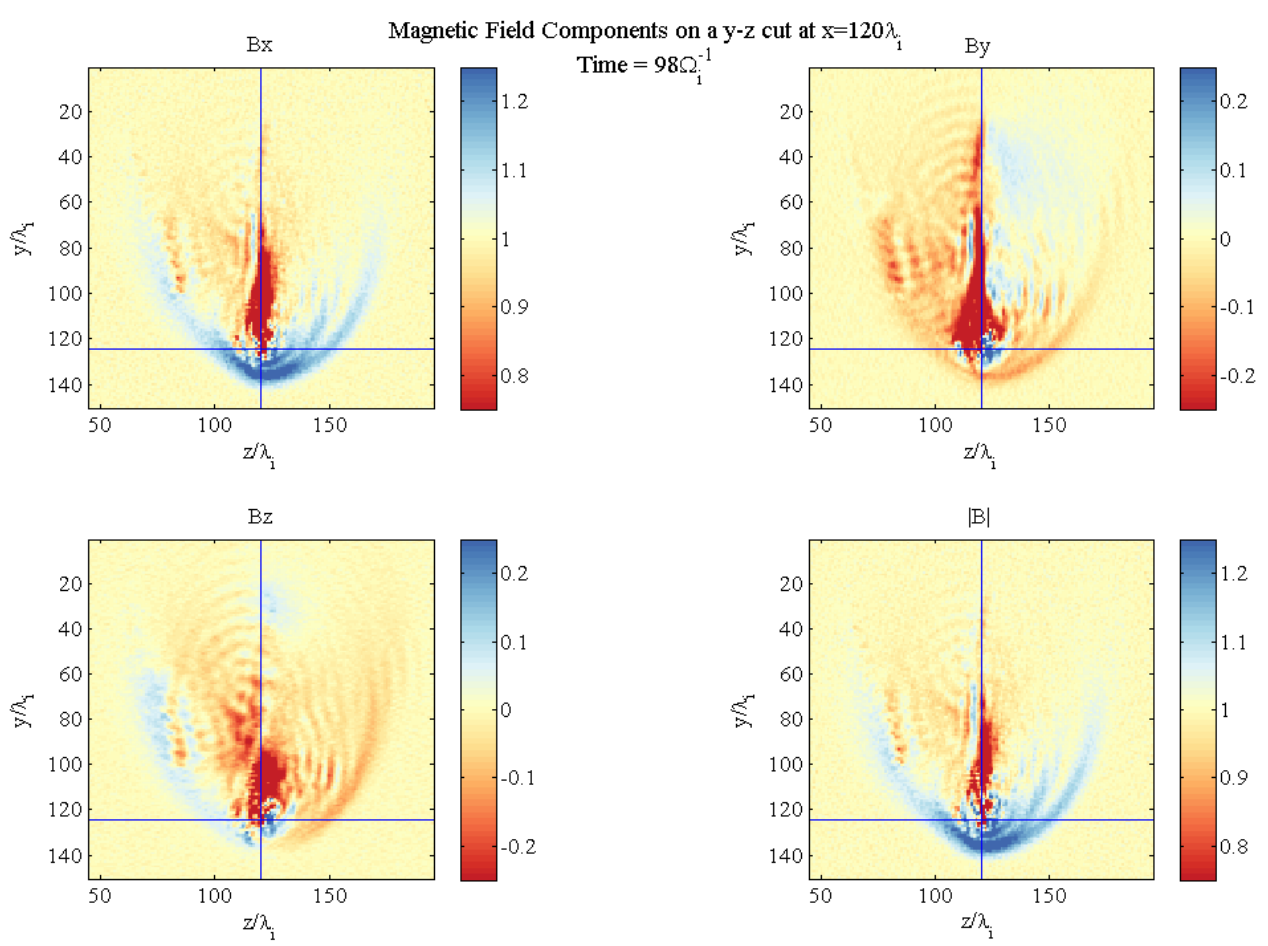

Figure 5.6.: The magnetic field on an $\hat{y}-\hat{z}$ plane at $\hat{x}=120 \lambda_{i}$ in the $45^{\circ}$ simulation domain after $98 \Omega_{i}^{-1}$. Signs of field line draping can be seen in the $B_{y}$ and $B_{z}$ components of the field as well as a region behind the spacecraft with a much reduced ambient field strength.

in the $+\hat{x}$ direction, the sense of the draping changes in $B_{y}$ as the field lines return to their undisturbed positions. This begins just ahead of the spacecraft as the field curves initially gently but the amount of curvature and hence the strength of the draping increases closer to the spacecraft. The draping is not restricted to just the $\hat{x}-\hat{y}$ plane or the $B_{y}$ component however as there is also evidence in $B_{z}$ which shows that the field also moves out in $+B_{z}$ to begin with as it simultaneously moves in $+B_{y}$ and then returns back to the same $\hat{x}-\hat{y}$ plane near to the spacecraft and in line with the spacecraft's $\hat{x}$ coordinate as the $B_{y}$ component also recovers but over a slightly wider region. Figure 5.6 shows a draping pattern in the $\hat{y}-\hat{z}$ plane and from this the extent of the draping patterns can also be seen. Field strengths in $B_{y}$ approach the $+30 \%$ level early on and then towards $-35-40 \%$ afterwards. The $B_{z}$ component has a very similar structure but is far weaker than that of the $B_{y}$, only reaching levels of $10-15 \%$ and occurring over a smaller spatial distance, the central region of negative field is roughly half the size of that in $B_{y}$. The draping in $B_{z}$ is not as expected as the field weaves in and out of this particular $\hat{x}-\hat{y}$ plane both throughout the interaction region and also for a time after it. 

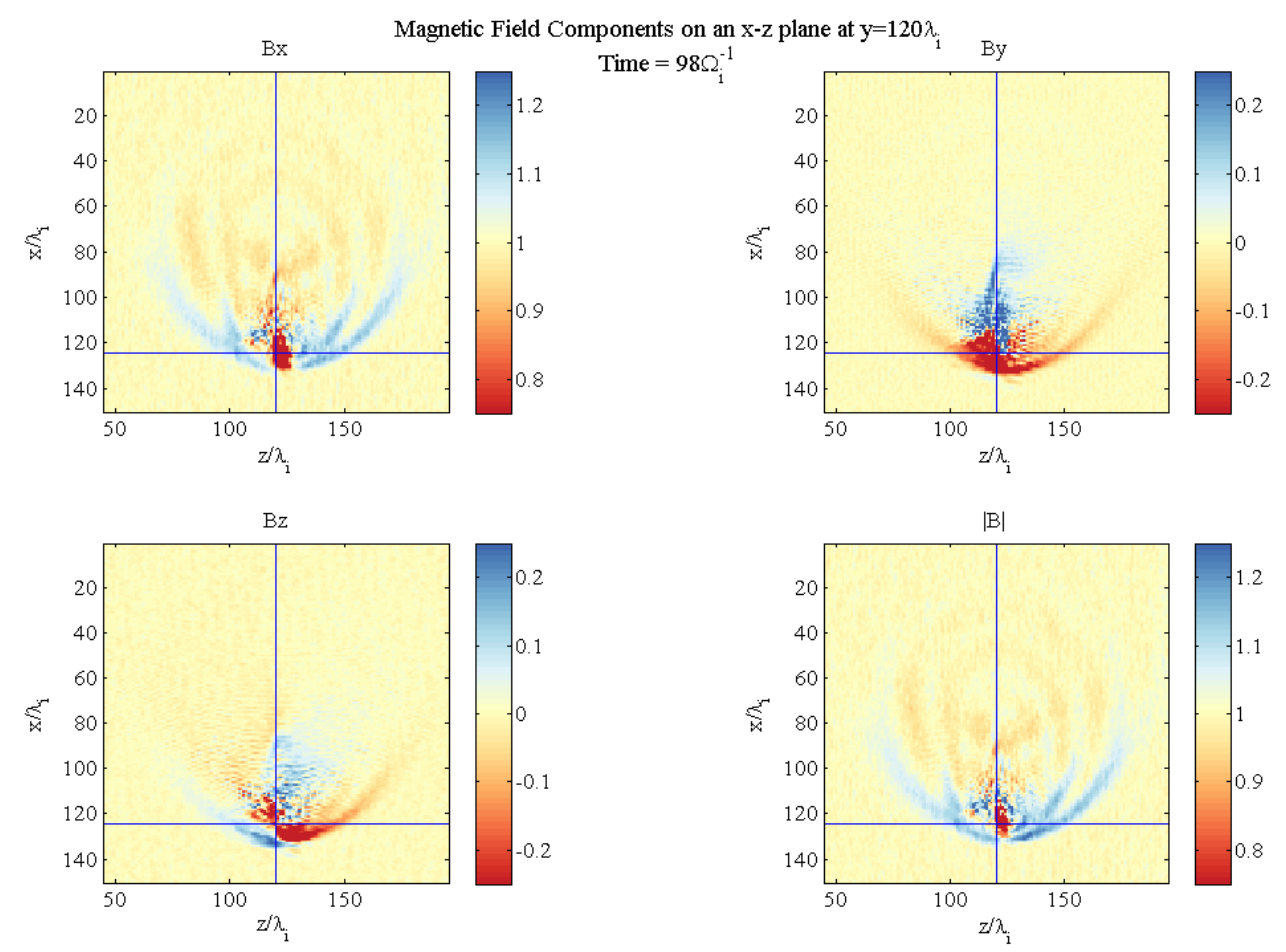

Figure 5.7.: The magnetic field on an $\hat{x}-\hat{z}$ plane at $\hat{y}=120 \lambda_{i}$ in the $45^{\circ}$ simulation domain after $98 \Omega_{i}^{-1}$. The draping along the $\hat{z}$ axis can be seen more clearly in this plane. The extent of the draping for the perpendicular components is reduced but the sense remains the same.

The draping begins modestly enough at early times and the first draping structure is formed with the strongest component at $+15 \%$ levels after $\approx 40 \Omega_{i}^{-1}$. Over the next $40 \Omega_{i}^{-1}$ the structure broadens and intensifies. This is due to the increasing radius of the injected ion cloud and the increasing effects of mass loading on the field.

Looking at the $\hat{x}-\hat{z}$ plane the picture changes a little as we can now see more clearly how the draping is altered along the $\hat{z}$ direction as Figure 5.7 shows. Ahead of the spacecraft (in this plane) the draping/field pattern follows the expected path as it is polarised to either side of the spacecraft in $B_{z}$ but is largely dominated by positive and then negative $B_{y}$ due to the bulk velocity of the cloud/spacecraft. The field also has a bulk draping profile within the interaction region as the $B_{y}$ component moves out of the plane and then back in along the $\hat{x}$ axis but also extending in the $-\hat{z}$ direction in a triangular pattern. The $B_{z}$ component shows a similar structure but is not as strong and does not cover as large of an area. 

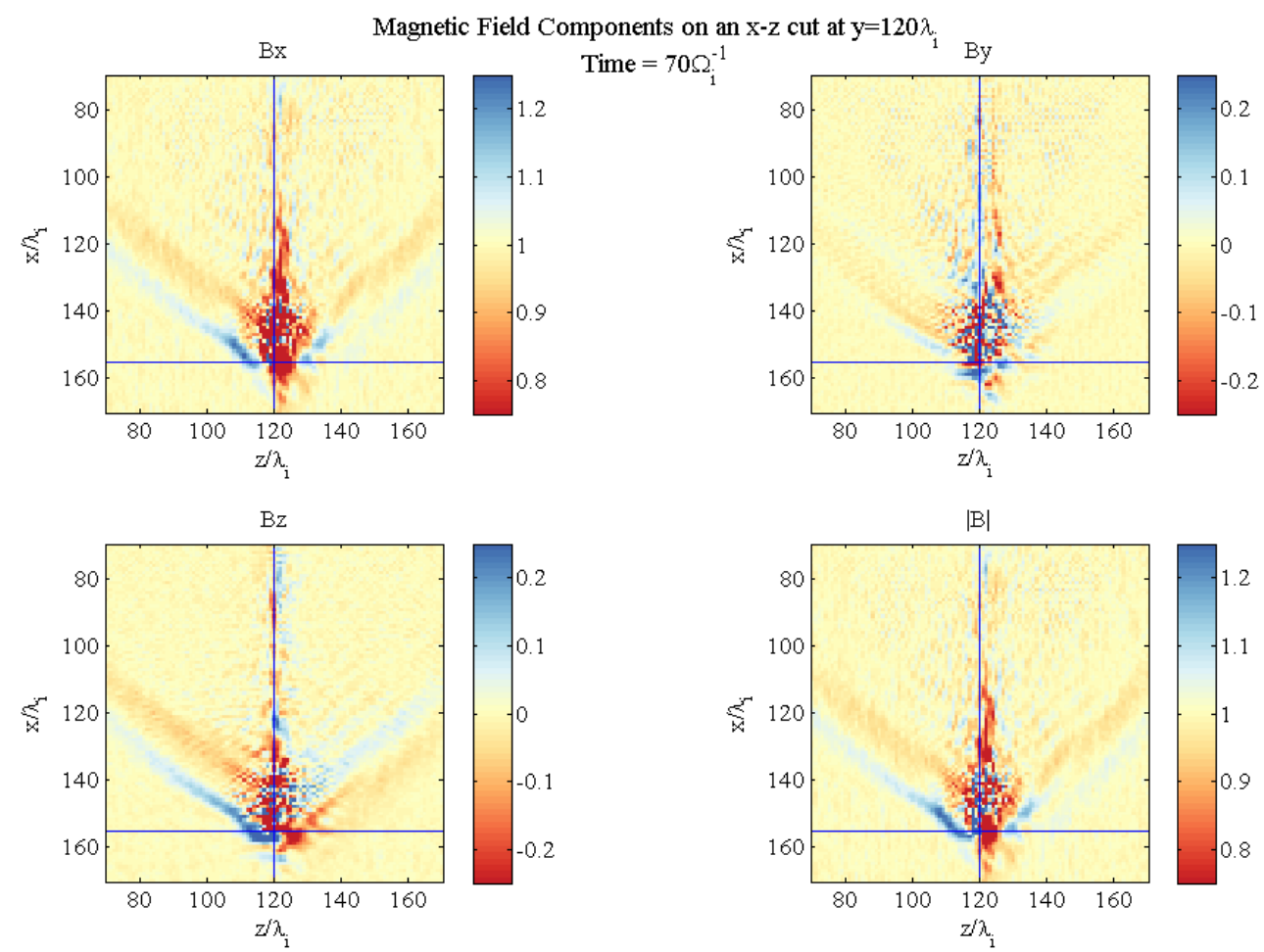

Figure 5.8.: The magnetic field on an $\hat{x}-\hat{z}$ plane at $\hat{y}=120 \lambda_{i}$ in the $0^{\circ}$ simulation domain after $70 \Omega_{i}^{-1}$. Whilst the field lines are not strictly 'draping' around the entire interaction region the field lines are being parted and curving around it by the motion and expansion of the ion cloud.

\subsubsection{Injection at $0^{\circ}$ to the Initial Magnetic Field}

The parallel injection simulation displays some signs of field draping but these are even less significant than for the $45^{\circ}$ case. As the cloud increases in size and density it causes the field lines to be pushed outward and away from the spacecraft which results in a draping pattern in the $\hat{x}-\hat{y}$ plane which is dominated by the effects in $B_{y}$ as the field lines are directly pushed away with very little out of plane movement, thus the $B_{z}$ component is very weak indeed, only reaching $5 \%$ values at the highest and often weaker. The extent of these effects is also confined to be much closer to the spacecraft.

As the simulation progresses the magnitude of the field line draping increases steadily with the size and density of the cloud. Similarly in the $\hat{x}-\hat{z}$ plane in Figure 5.8 we see a similar pattern but rotated into the appropriate plane. The $B_{y}$ component is now very weak and the $B_{z}$ component shows the main features of the field line draping in similar fashion to the $B_{y}$ component in the $\hat{x}-\hat{y}$ plane. This shift occurs gradually as the plane rotates from $\hat{x}-\hat{y}$ to $\hat{x}-\hat{z}$ and the components are bal- 
anced in strength at a $45^{\circ}$ rotation from $\hat{x}-\hat{y}$ towards $\hat{x}-\hat{z}$. Figure 5.3 in section 5.2 .2 shows a view perpendicular to Figure 5.8 with stronger effects in the $\hat{y}$ direction. The $\hat{y}-\hat{z}$ plane shows the same picture, that of field lines running towards the cloud and being diverted around it. Additionally the rotation of the draping can be more easily seen in this plane.

\subsubsection{Low Injection Rate at $90^{\circ}$ to the Initial Magnetic Field}

The draping effects for the low injection rate simulation manifest almost immediately but at very low levels of $5 \%$ of the strength of the undisturbed magnetic field. The form of the draping is the same as the reference simulation but with weaker effects. These effects begin subtly and increase over the duration of the simulation from $5 \%$ levels to $25 \%$ levels of the strength of the undisturbed magnetic field. Figure 5.9 shows the magnetic field late in the simulation where the draping effects have reached large values. As the cloud expands the effect of the draping increases in both strength and extent of effect. There is no draping in the main core of the cloud and in fact it is field free to some extent.

In the $\hat{x}-\hat{z}$ plane the draping effects again show an asymmetry in the $\hat{z}$ direction as well as a triangular structure along the $\hat{x}$ direction in the same manner as previous simulations within the region bounded by the main draping structure. The draping in the $\hat{y}-\hat{z}$ plane is also very similar to the reference simulation in form with very little effect being noticeable at the central plane and only very mild fluctuations being observed. Overall in this particular plane the draping effects are very minor.

\subsubsection{High Injection Rate at $90^{\circ}$ to the Initial Magnetic Field}

The relatively high injection rate used for this simulation results in the strongest draping fields of all of the simulations performed. The $B_{y}$ and $B_{z}$ components of the magnetic field very quickly reach $50 \%$ levels of fluctuations. The extent of the stronger draping pattern is also far larger than for the other simulations, extending over $50 \lambda_{i}$ as compared to as little as $10-20 \lambda_{i}$ in other simulations.

Further, the actual draping pattern also changes slightly for this simulation. For previous simulations the field, running along $+\hat{x}$, would first be deflected simultaneously in $+\hat{y}$ and $+\hat{z}$ before then reversing once past the spacecraft in order to return to the proper plane. Here the $B_{z}$ component shows some 'rippling' before the major 

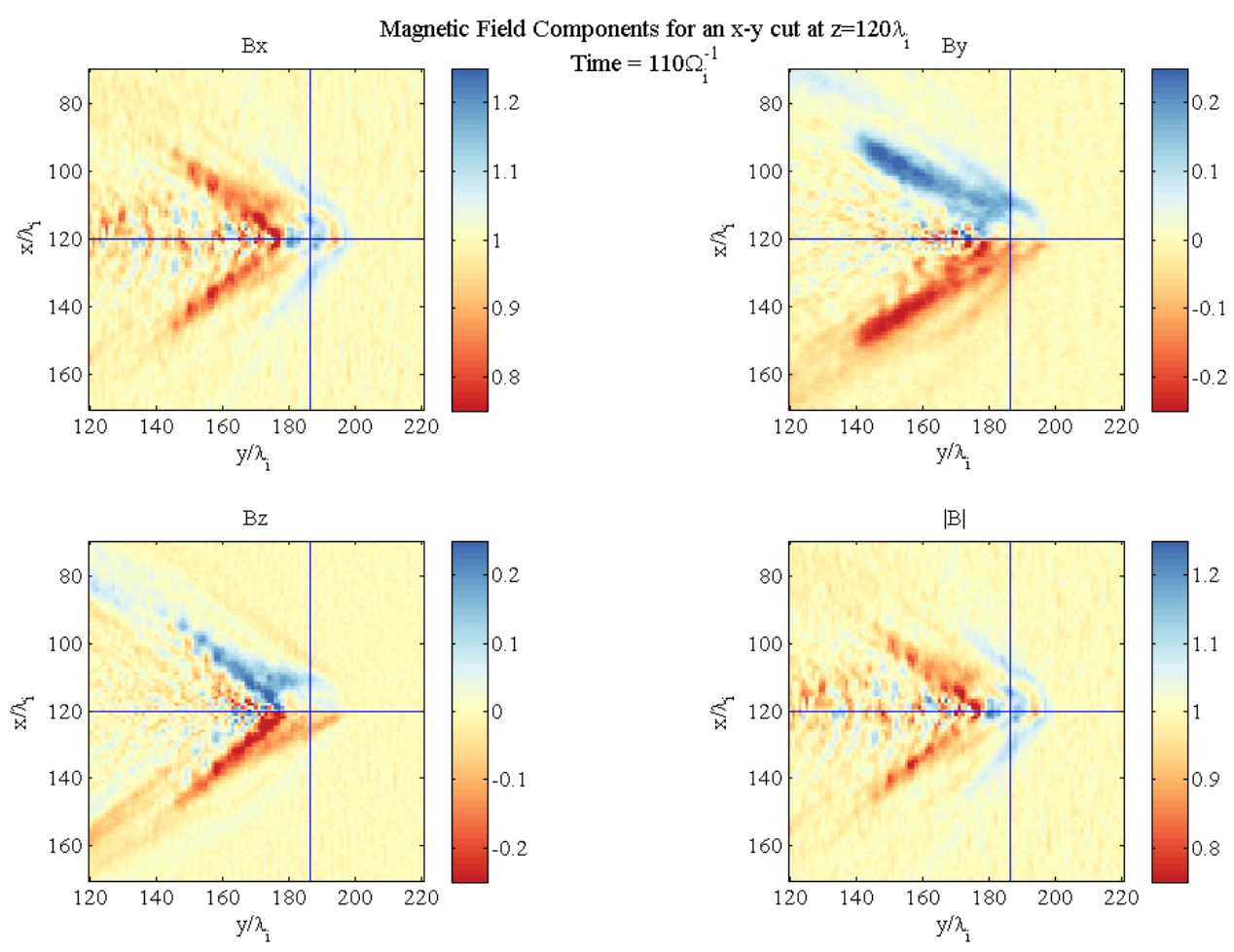

Figure 5.9.: The magnetic field on an $\hat{x}-\hat{y}$ plane at $\hat{z}=120 \lambda_{i}$ in the low injection rate simulation domain after $110 \Omega_{i}^{-1}$. Signatures of field line draping can be seen although they are weaker than in other simulations whilst qualitatively similar. Injection is at $90^{\circ}$ to the initial magnetic field direction. 

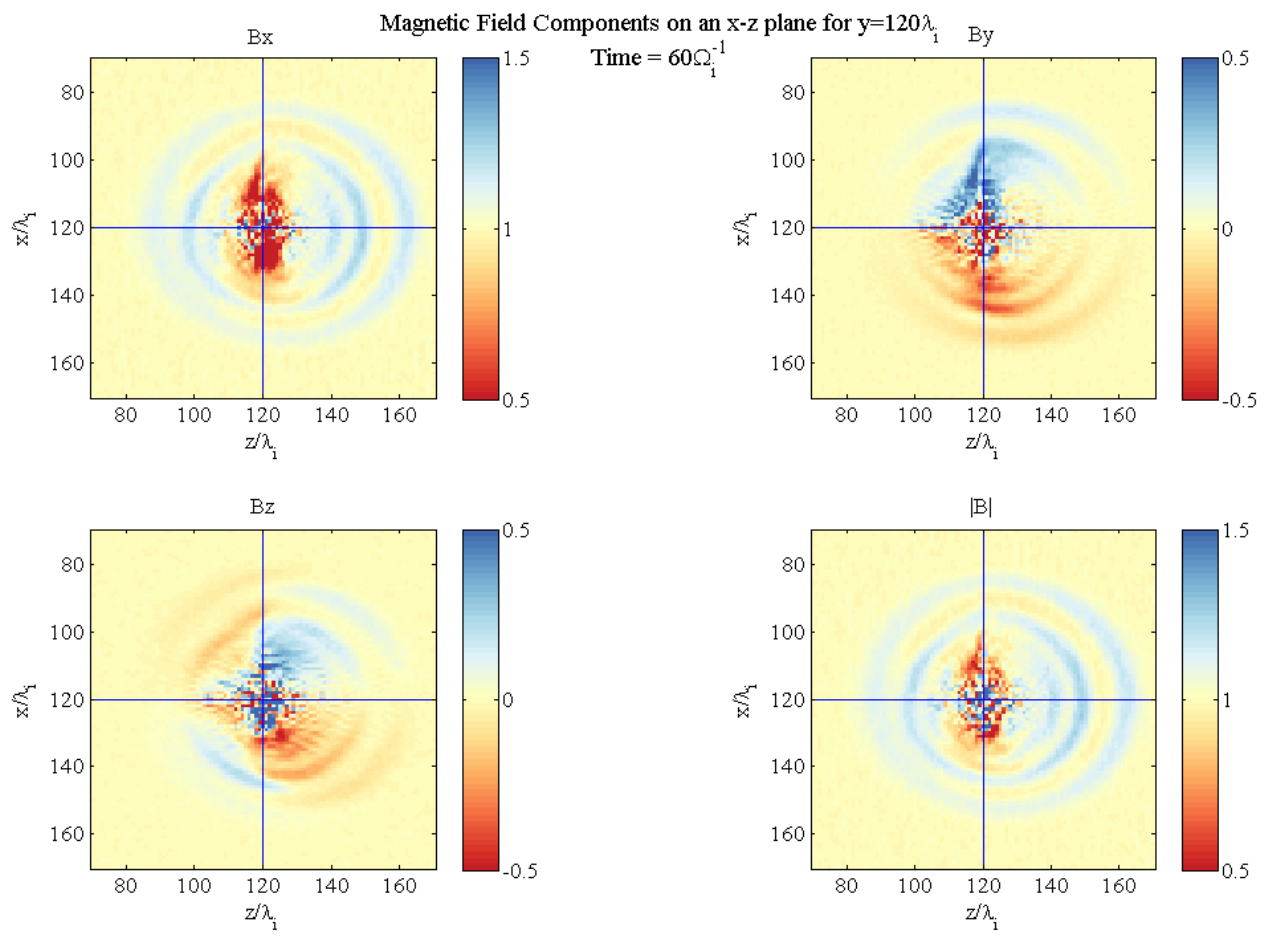

Figure 5.10.: The magnetic field on an $\hat{x}-\hat{z}$ plane at $\hat{y}=120 \lambda_{i}$ in the high injection rate simulation domain after $60 \Omega_{i}^{-1}$. The magnetic field can be seen to drape around the spacecraft by the oppositely directed field at either side of the spacecraft. In this plane the spacecraft is travelling into the page. Injection is at $90^{\circ}$ to the initial magnetic field direction. 
draping occurs. These ripples are also associated with additional perturbations in $B_{y}$ but thinner and with a lower amplitude than the central draping feature. This additional structure is not observed in other simulations. The effect is so large, however, that the effects extend a significant distance into the core of the cloud. The $\hat{x}-\hat{z}$ plane shows clearer signs of field line draping as the $B_{y}$ field is polarised along the $\hat{x}$ direction and the $B_{z}$ component is polarised in both the $\hat{x}$ and $\hat{z}$ directions as seen in Figure 5.10, additional field line draping features can be seen in Figure 5.5 in section 5.2.4.

\subsection{Bow Waves}

This section will look at the bow waves formed by the thruster firings, paying attention to both the magnetic component and also to the signature in the number densities as the solar wind plasma is disturbed.

\subsubsection{Injection at $45^{\circ}$ to the Initial Magnetic Field}

The bow wave formed at this angle of injection takes approximately $40 \Omega_{i}^{-1}$ to form as the density of the injected cloud increases as can be seen from Figure 5.11. In contrast to the pileup of the magnetic field lines in the $\hat{x}-\hat{y}$ plane the bow wave forms most strongly towards the $+\hat{x}$ direction as opposed to the pileup which dominates in the $+\hat{y}$ direction.

The bow wave observed begins to form after $40 \Omega_{i}^{-1}$ and then develops over the following $40 \Omega_{i}^{-1}$ to form a wave front which is predominantly in the $+\hat{x}$ direction with a small part along the $+\hat{y}$ direction. In this plane the bow wave extends for over $100 \lambda_{i}$ and reaches amplitudes of $50 \%$ over the course of the simulation. Figure 5.12 shows that by the end of the simulation multiple wave fronts can be seen to form. Much more minor waves at the $10 \%$ level can also be seen propagating in the $+\hat{y}$ direction, coincident with the magnetic pileup. Further, an asymmetry along the $\hat{y}$ direction can also be seen, this is due to the ability of the ions to stream more freely along the direction parallel to the magnetic field which results in the bow wave existing for a longer duration in comparison to the perpendicular direction where the Lorentz force causes ion gyration and hence dissipation of the wave.

Consideration of the $\hat{x}-\hat{z}$ plane shows that the bow wave is roughly symmetric over $\hat{z}$ and is able to propagate away from the spacecraft and spread out, dispersing as it does so. The $\hat{y}-\hat{z}$ plane shows the same picture as the cloud and spacecraft move into the plane with a moderate enhancement ahead of it which then gives way 


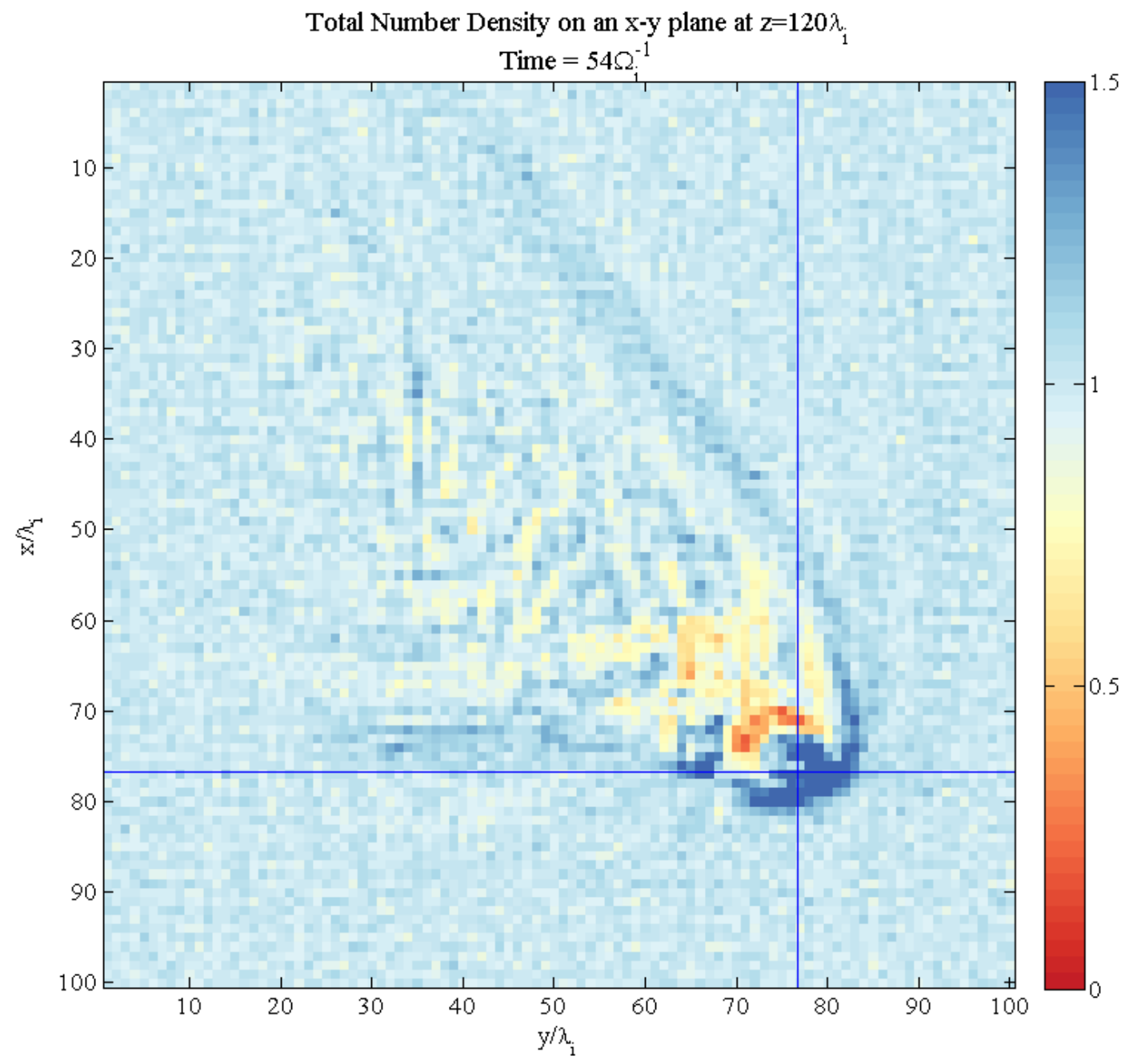

Figure 5.11.: The total number density of all species on an $\hat{x}-\hat{y}$ plane at $\hat{z}=120 \lambda_{i}$ in the $45^{\circ}$ simulation domain after $54 \Omega_{i}^{-1}$. The beginnings of the bow wave formation can be seen. 


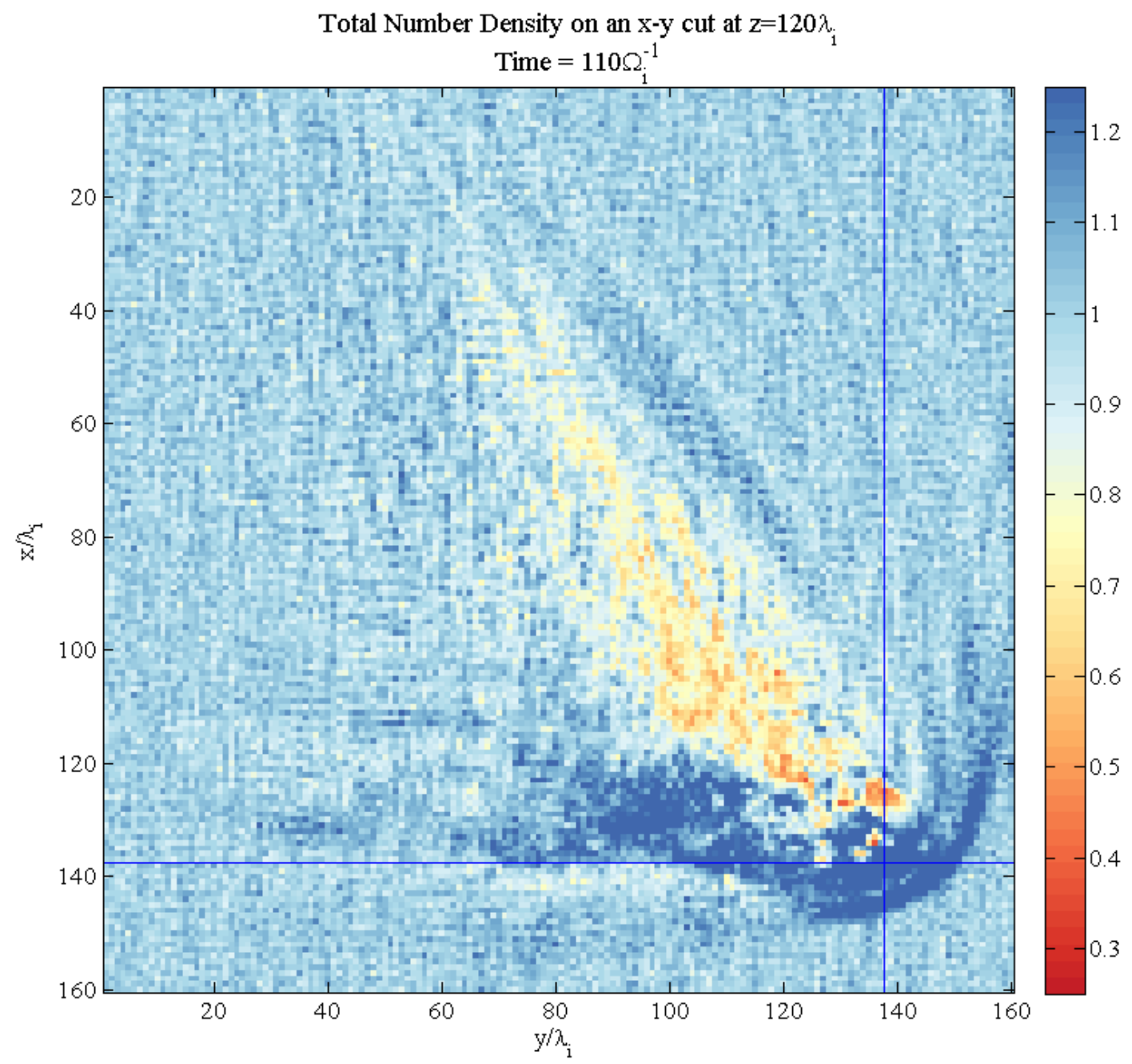

Figure 5.12.: The total number density of all species on an $\hat{x}-\hat{y}$ plane at $\hat{z}=120 \lambda_{i}$ in the $45^{\circ}$ simulation domain after $110 \Omega_{i}^{-1}$. By this time the bow waves have more fully formed and a pronounced peak can be seen ahead of the spacecraft's position with an asymmetry along the $\hat{y}$ axis. 

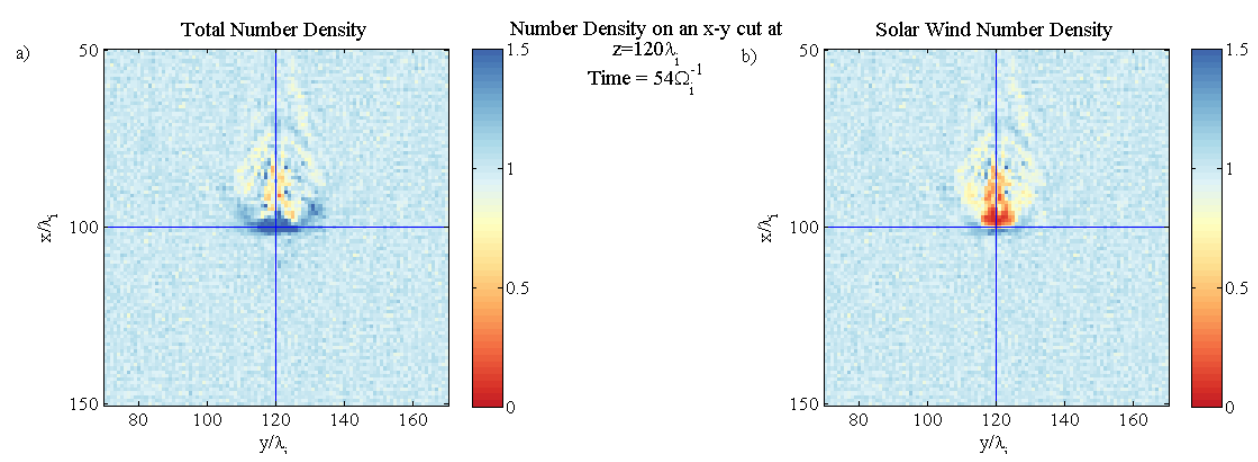

Figure 5.13.: a) The total number density of all species on an $\hat{x}-\hat{y}$ plane at $\hat{z}=120 \lambda_{i}$ in the $0^{\circ}$ simulation domain after $54 \Omega_{i}^{-1}$. b) The solar wind number density on an $\hat{x}-\hat{y}$ plane at $\hat{z}=120 \lambda_{i}$ in the $0^{\circ}$ simulation domain after $54 \Omega_{i}^{-1}$. A modest bow wave is formed but is composed almost entirely of injected ions, the solar wind component of is shown to be extremely weak.

to the induced interaction region of the cloud and an asymmetric (in $\hat{z}$ ) bow wave of the same size and amplitude as in the $\hat{x}-\hat{z}$ plane.

\subsubsection{Injection at $0^{\circ}$ to the Initial Magnetic Field}

A strong bow wave is not formed in this simulation in a similar manner to the magnetic pileup as a result of the injection angle being parallel to the magnetic field. The highest density of ions is the cloud core and as field lines are not being swept up by it nor is a great deal of the solar wind plasma. Towards the end of the simulation a very small bow wave, broken in the near-parallel direction, forms with an amplitude of only 10-15\%. The main mechanism for this sweeping up of even small amounts of the solar wind plasma is the expansion of the core of the ion cloud. This causes some of the solar wind plasma to be accelerated along their field lines and cause minor enhancements to the solar wind density ahead of the ion cloud forming a weak bow wave.

Along the $\hat{z}$ direction in the $\hat{x}-\hat{z}$ plane a bow wave can more clearly be seen to form. This begins to form early on in the simulation, after $45 \Omega_{i}^{-1}$. The amplitude of the bow wave increases from $10 \%$ to $40 \%$ levels over the simulation. As the region of influence of this bow wave spreads along the $\pm \hat{z}$ direction a region behind the wave forms which is depleted of solar wind plasma. Panel a) of Figure 5.13 shows the formation of a density pulse ahead of the spacecraft of a reasonable strength whilst panel b) of Figure 5.13 which shows only the solar wind density indicates that only a small number of solar wind protons are actually being swept up by the injected 
ion cloud.

The $\hat{x}-\hat{y}$ plane shows almost no evidence of a fully formed bow wave. As the cloud passes through there is very little disturbance and only a very weak wave is seen to propagate a short distance before dispersing.

\subsubsection{Low Injection Rate at $90^{\circ}$ to the Initial Magnetic Field}

As the injection rate is much lower for this simulation a strong bow wave was neither expected nor observed. However, a modest bow wave is formed gradually with peak amplitudes approaching the $30 \%$ level as shown in figure 5.14. The waves seen are of the same shape as those seen in the reference simulation but generally weaker as the solar wind is more readily able to accommodate the addition of this lesser mass and thus is less disturbed.

Additionally, whereas before multiple wave fronts were formed ahead of the spacecraft, in this case there is only ever a single well formed wave front present at any given time as the leading wave fronts disperse by the time any subsequent waves form. The $\hat{x}-\hat{z}$ plane, again, shows little in the way of perturbations. As the cloud passes through the plane a weak pulse is launched with amplitudes of only $10 \%$. There is an asymmetry in this towards the $-\hat{z}$ direction due to the ion gyration. This produces something akin to a secondary bow wave. All these perturbations quickly disperse. As expected the $\hat{y}-\hat{z}$ plane also shows little evidence of a fully formed bow wave. Towards the end of the simulation there is a very small, weak bow wave forming.

\subsubsection{High Injection Rate at $90^{\circ}$ to the Initial Magnetic Field}

Also as expected this high injection rate simulation very quickly clears out a region of solar wind plasma to form a bow wave ahead of the spacecraft. After only $15 \Omega_{i}^{-1}$ the beginning of the wave can be seen where it then rapidly expands and strengthens as the solar wind is unable to deal with the level of mass loading. Contrasting with the low injection rate simulation where no additional pulses were launched ahead of the spacecraft, during this simulation several wave fronts were observed at once and potentially more may have formed behind those as the first pulse was still of a significant amplitude. Figure 5.15 shows the formation of multiple wave fronts in the bow wave. 


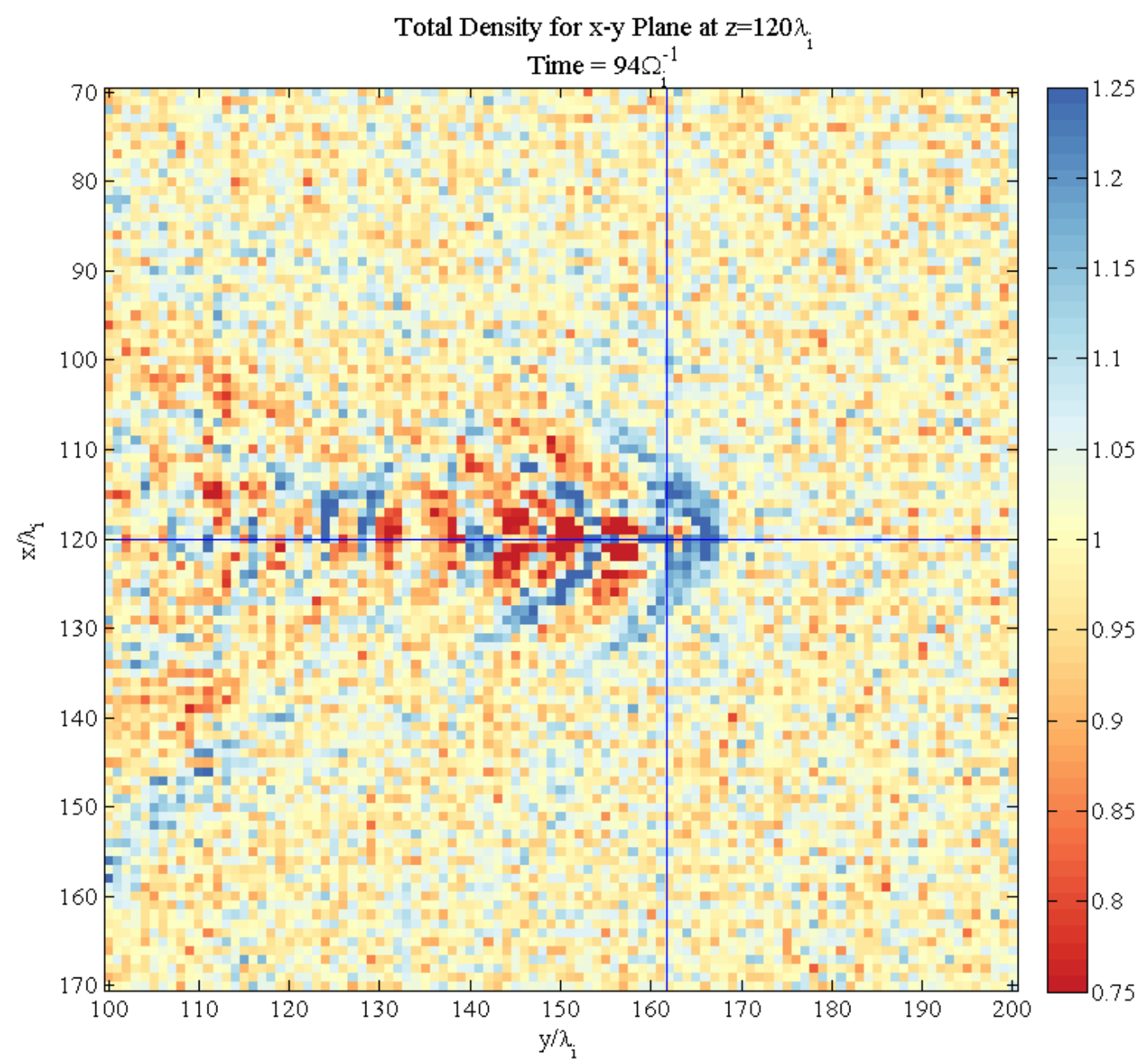

Figure 5.14.: The total number density of all species on an $\hat{x}-\hat{y}$ plane at $\hat{z}=120 \lambda_{i}$ in the low injection rate simulation domain at $90^{\circ}$ to the initial magnetic field after $94 \Omega_{i}^{-1}$. Even late into the simulation only a very weak bow wave is formed due to the low injection rate, the shape remains qualitatively the same as the other $90^{\circ}$ simulations however. Injection is at $90^{\circ}$ to the initial magnetic field direction. 


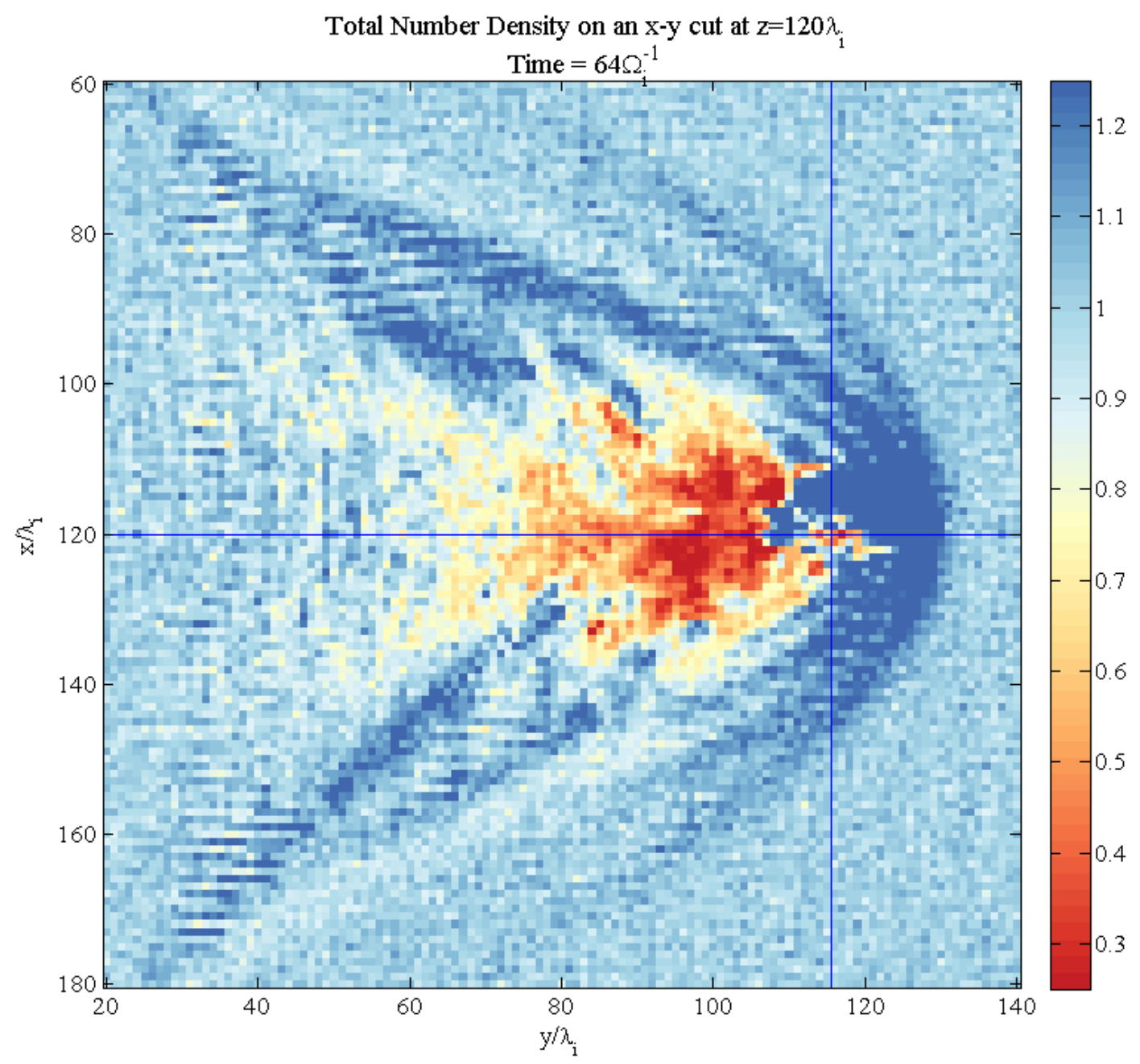

Figure 5.15.: The total number density of all species on an $\hat{x}-\hat{y}$ plane at $\hat{z}=120 \lambda_{i}$ in the high injection rate simulation domain at $90^{\circ}$ to the initial magnetic field after $64 \Omega_{i}^{-1}$. Several very strong bow waves have formed and merged into a single pulse in the ram direction. The high density ion cloud can also be seen ahead of the spacecraft. Injection is at $90^{\circ}$ to the initial magnetic field direction. 
Similarly in the $\hat{x}-\hat{z}$ and $\hat{y}-\hat{z}$ planes the multiple wave fronts are clearly visible and very high in amplitude. There is also an asymmetry in the $\hat{z}$ direction due in part to gyration of ions and in part to the expansion of the cloud. The bow waves in this run grow from only $10 \lambda_{i}$ to over $100 \lambda_{i}$ in length. Further, directly ahead of the spacecraft the subsequent bow waves have a sufficiently high phase speed that they are able to catch up with previously produced pulses to form an extended high density leading pulse.

\subsection{Interaction Region}

In each simulation, due to the loading of mass onto the solar wind flow, an induced interaction region is formed. We define this region to be that volume of the simulation in which the ions injected as a result of the thruster firing have had a significant effect upon the solar wind and beyond which the solar wind remains, to a large extent, undisturbed or recovered. This region holds the majority of the newly injected material and its size, shape and depth can vary with the solar conditions as well as the size of the thrust.

\subsubsection{Injection at $45^{\circ}$ to the Initial Magnetic Field}

The interaction region begins to form in as little as $10 \Omega_{i}^{-1}$ but does not start to expand significantly until $45 \Omega_{i}^{-1}$ into the simulation. In this case the interaction region is formed of two parts, a magnetic component and a density component.

The magnetic component of the interaction region is formed early on and grows throughout the simulation. The extent of this magnetic component can be seen in Figure 5.1 in section 5.2.1. This can be seen as a region of reduced $|B|$ which forms and then expands perpendicular to the ambient field whilst itself still travelling parallel to the field. The result of this is a region which extends for $70 \lambda_{i}$ along the $\hat{y}$ axis whilst still extending for $25 \lambda_{i}$ in the $\hat{x}$ direction.

The density component of the interaction region is not as extensive as for the magnetic field. A roughly spherical region is formed of approximately $10 \lambda_{i}$ radius inside which the solar wind protons are significantly depleted in number density as displayed in Figure 5.16. This is inside the region of space where the injected ion cloud lies. Further, this interaction region displays a 'tail' of material left behind it. Solar wind density levels are also reduced in this region and its effects extend for $140 \lambda_{i}$ behind the spacecraft.

The main body of the interaction region is bounded by magnetic pileup on one 


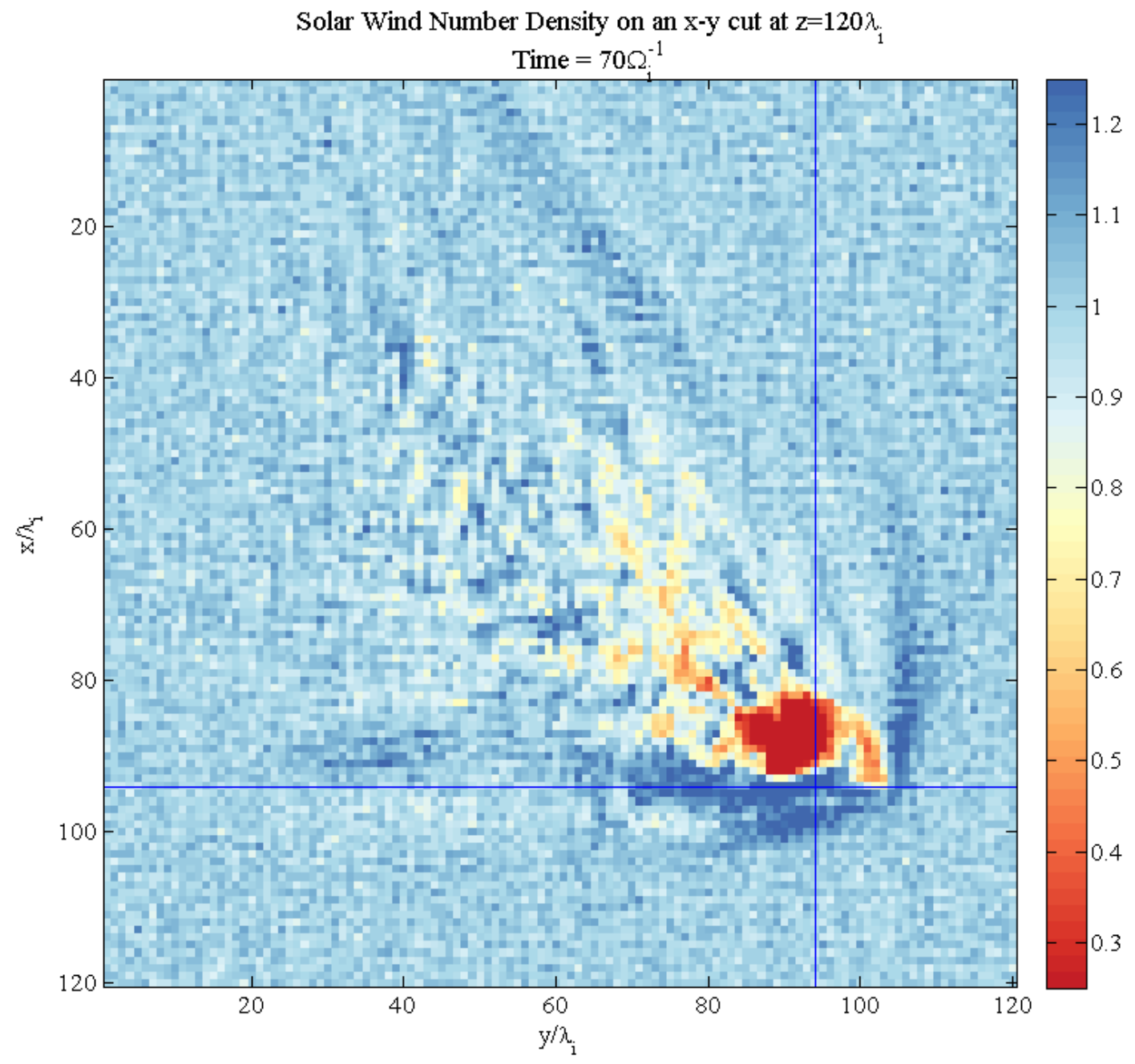

Figure 5.16.: The solar wind number density on an $\hat{x}-\hat{y}$ plane at $\hat{z}=120 \lambda_{i}$ in the $45^{\circ}$ simulation domain after $70 \Omega_{i}^{-1}$. The beginnings of the formation of the interaction region's core can be seen as the solar wind number density becomes heavily depleted in the region of the injected ion cloud. 
side and then by a density bow wave on the other with respect to the ram direction with a smooth transition region directly ahead of the spacecraft where the pileup of flux is reduced and the density of the solar wind is increasing. The interaction region exists primarily in the $\hat{x}-\hat{y}$ plane but does also have a presence in the $\hat{y}$ - $\hat{z}$ plane although its width is only a few $\lambda_{i}$ in the $\hat{z}$ direction.

\subsubsection{Injection at $0^{\circ}$ to the Initial Magnetic Field}

For the case of parallel injection the interaction region is primarily magnetic in nature although there is a small region where the solar wind density becomes depleted extending $20 \lambda_{i}$ across $\hat{y}$ and $50 \lambda_{i}$ along $\hat{x}$. There also exists a region behind the spacecraft where there is an ion tail. Again this results in a region of space in which the solar wind density is slightly reduced and extends $125 \lambda_{i}$ behind the spacecraft. This interaction region is bounded in the ram direction by a slight enhancement to density: a weak bow wave. It can be seen in Figure 5.17 that the density component of the interaction region is relatively small. It is bounded in the $\hat{y}$ and $\hat{z}$ directions by weak magnetic waves.

The magnetic 'cavity' region is far larger than the density reduced region as shown by Figure 5.18. The magnitude of the field is reduced in this region. The field within this interaction region, whilst reduced with respect to the ambient field, is still turbulent in the $B_{y}-B_{z}$ components as there is no overall average direction in which the field there is oriented, however, this is also true of the other simulations.

The interaction region in this simulation has a unique feature compared to the others. As the injection is parallel to the field injected particles are able to stream freely ahead of the spacecraft. Only a relatively small number of particles are able to achieve this in practice due, in part, to scattering effects which are present. The particles which are able to travel ahead of the position of the main bow wave cause a minor disturbance to the magnetic field. Over the duration of the simulation this interaction grows from a mild fluctuation into a region where the ambient field has dropped to levels comparable with the main cavity. This effect can be seen by the magnetic fluctuations ahead of the spacecraft in Figure 5.18.

The main body of the interaction region has a relatively flat profile in the ram direction, mimicking the shape of the ion cloud. The scattering and streaming of particles is not limited to along the $+\hat{x}$ direction as particles are also backscattered to travel in the $-\hat{x}$ direction. These particles quickly become disconnected from the main body of the interaction region but their influence is still significant as the small clumps of ions are able to cause the ambient solar wind magnetic field to drape 


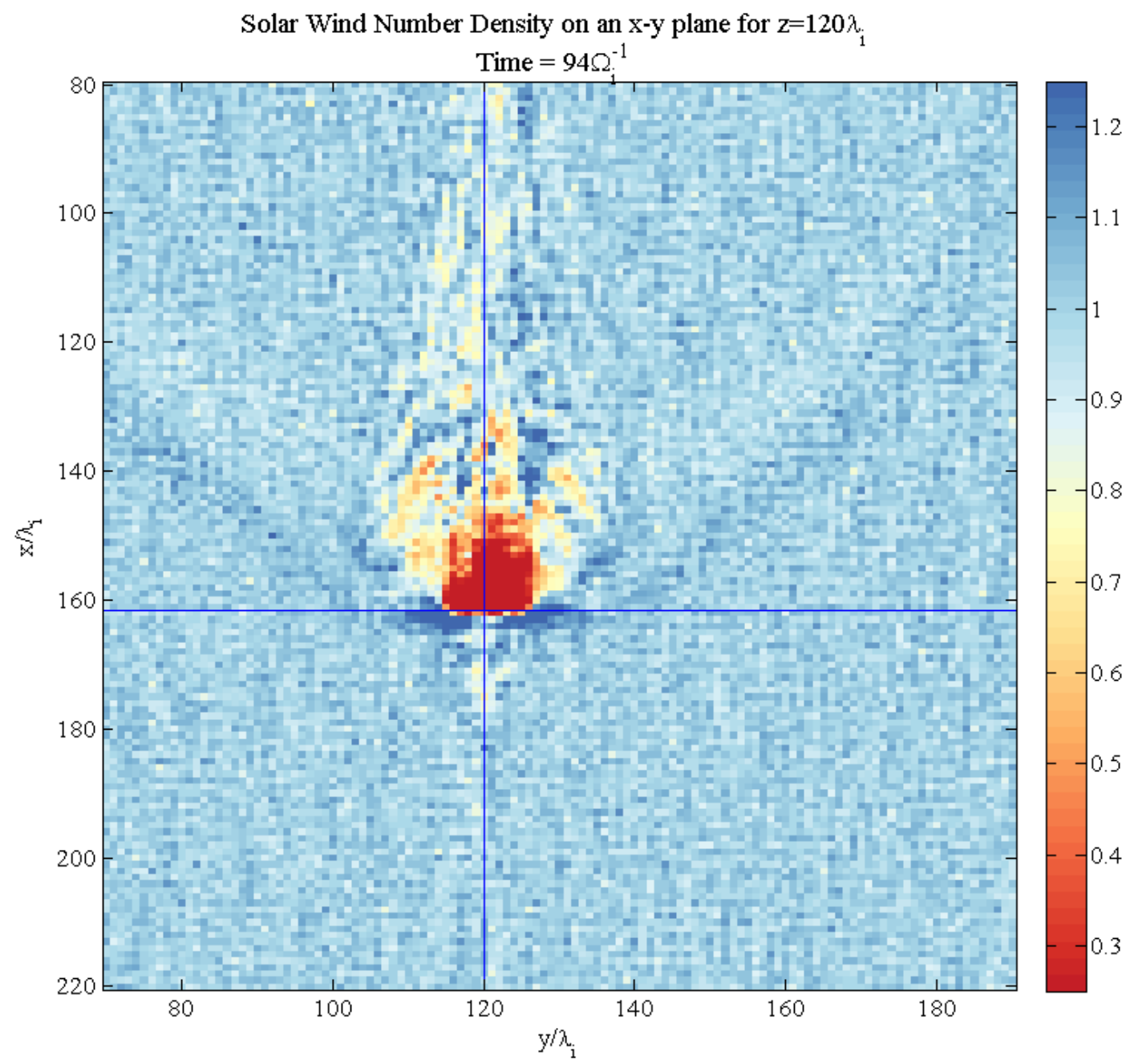

Figure 5.17.: The solar wind number density on an $\hat{x}-\hat{y}$ plane plane at $\hat{z}=120 \lambda_{i}$ in the $0^{\circ}$ simulation domain after $94 \Omega_{i}^{-1}$. The formation of the density component of the interaction region can be seen forming. It is far smaller than the magnetic component but the levels of solar wind protons in the core region drops almost to nothing. 

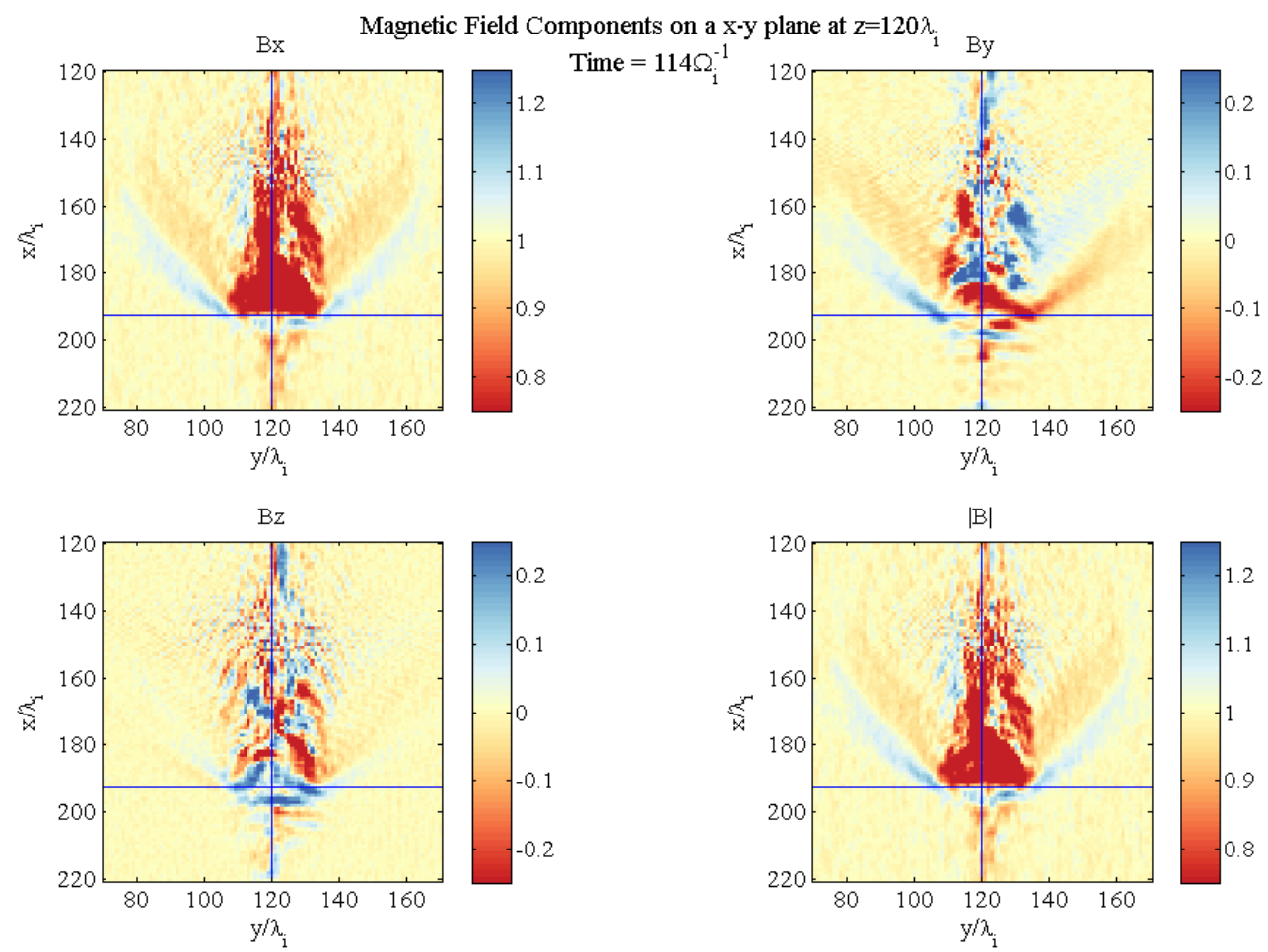

Figure 5.18.: The magnetic field components on an $\hat{x}-\hat{y}$ plane at $\hat{z}=120 \lambda_{i}$ in the $0^{\circ}$ simulation domain after $114 \Omega_{i}^{-1}$. The extent of the magnetic interaction region extends a far greater distance than the density component. Wider along the $\hat{y}$ and $\hat{z}$ axes it also extends a great distance behind the spacecraft with relatively strong effects as the field strength drops by a modest amount. 
around them. Thus the interaction region in this case is not restricted to only the main core of the ion cloud but also extends some distance both ahead and behind due to fast streaming and scattered ions.

\subsubsection{Low Injection Rate at $90^{\circ}$ to the Initial Magnetic Field}

The interaction region in the low injection rate simulation is not as well formed as those of other simulations. In terms of the volume cleared, even partially, of solar wind ions its influence is small as displayed in Figure 5.19. In this case the density perturbations of the inner cloud produce a larger area of effect at their maximum than do the magnetic fluctuations. The interaction region, in terms of magnetic effects, is small also and is defined largely by the relatively minor effects of the cloud's tail.

These effects are rarely large and are confined to the region behind the spacecraft and fairly close to its trajectory. The rate of mass loading in this simulation is simply not sufficient to see the formation of a stable interaction region over the duration observed.

\subsubsection{High Injection Rate at $90^{\circ}$ to the Initial Magnetic Field}

Whilst the $45^{\circ}$ injection angle simulation showed that the interaction region is affected by the injection geometry and can be larger than a strictly $90^{\circ}$ injection, the size of the interaction region is dominated by the amount of mass loaded onto the solar wind. The interaction region is formed extremely quickly, within $5 \Omega_{i}^{-1}$ and from there only continues to grow as more mass loading occurs. Magnetically, the interaction region spans a width parallel to the magnetic field of $40 \lambda_{i}$ and extends from the starting position of the spacecraft all the way to the spacecraft, over $100 \lambda_{i}$. The main body of the interaction region, however, excluding the ion tail is a little more compact, forming a rough sphere of radius $25 \lambda_{i}$ as seen in Figure 5.20. The magnetic depressions formed though make a diamagnetic cavity in the region close to the spacecraft extending $60 \lambda_{i}$ by $25 \lambda_{i}$.

Additionally the mass loading is severe enough that the wings formed by the field draping have a significantly reduced field strength also, although the field does not completely disappear. As there is such a large effect on the magnetic field it is unsurprising that there is also a very large region which is cleared either partially or 


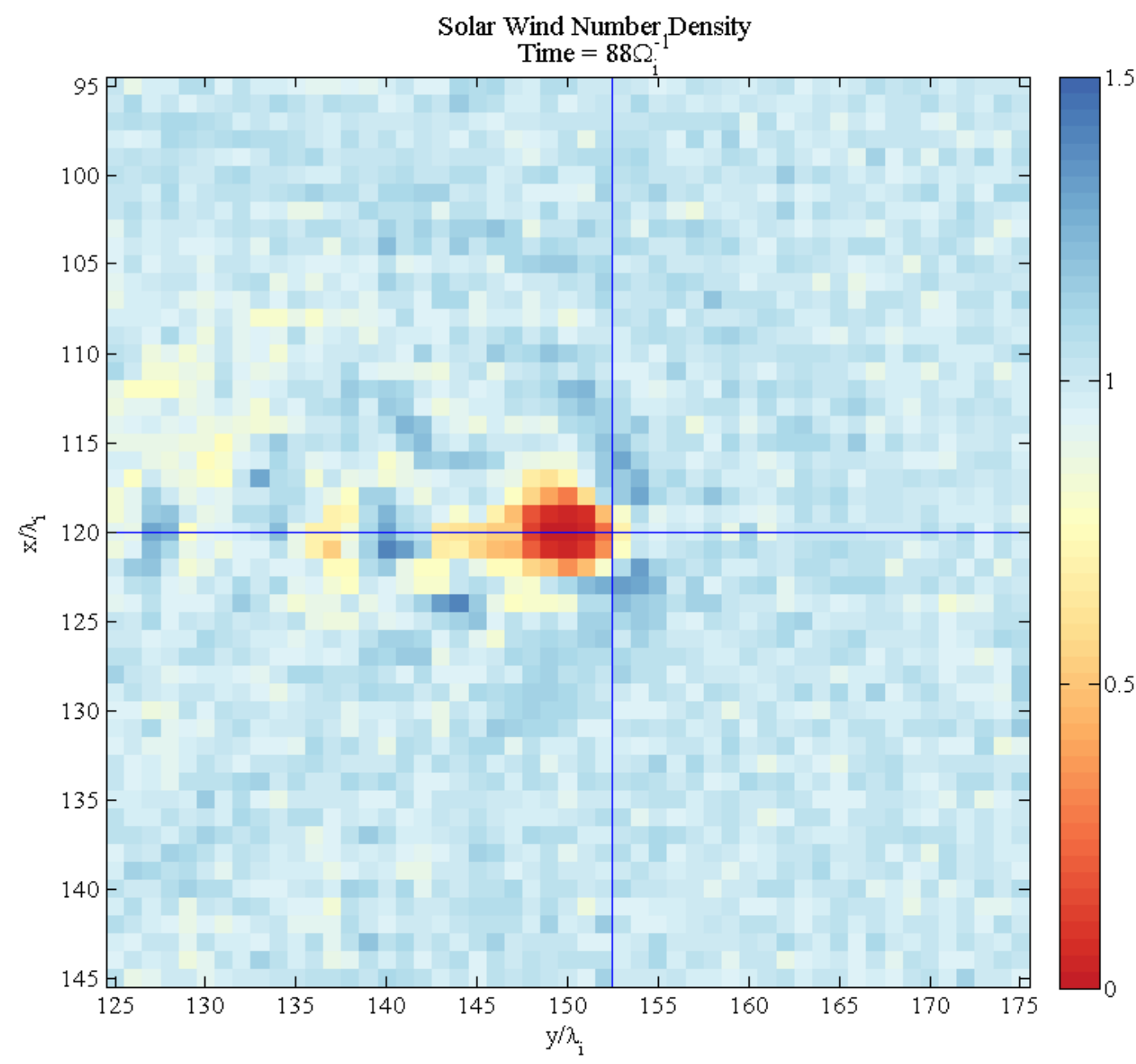

Figure 5.19.: The solar wind number density on an $\hat{x}-\hat{y}$ plane at $\hat{z}=120 \lambda_{i}$ in the low injection rate simulation domain at $90^{\circ}$ to the initial magnetic field after $88 \Omega_{i}^{-1}$. The interaction region formed by this very low injection rate is correspondingly small although its depth remains competitive with the higher injection rate simulations. Injection is at $90^{\circ}$ to the initial magnetic field direction. 


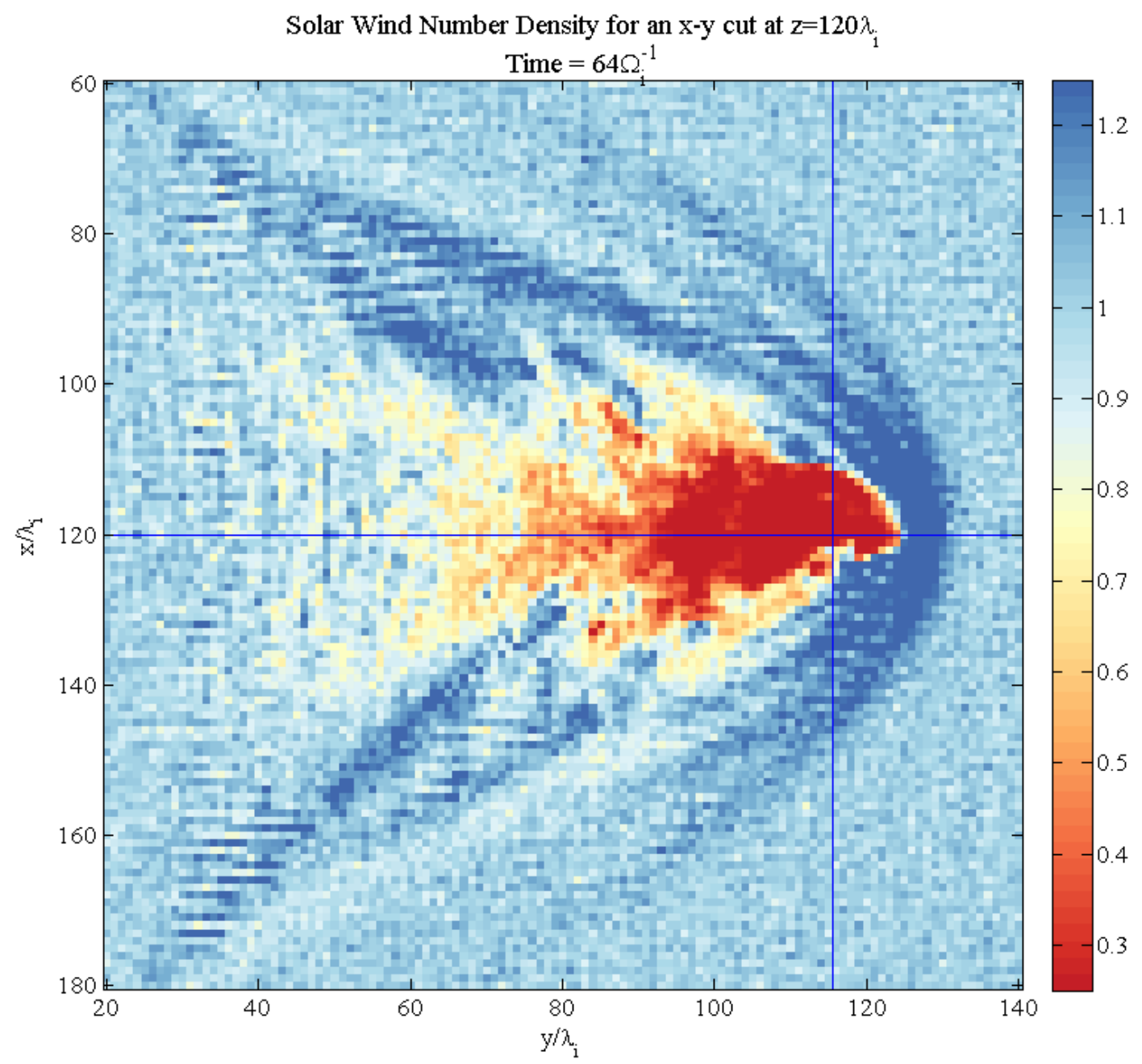

Figure 5.20.: The solar wind number density on an $\hat{x}-\hat{y}$ plane at $\hat{z}=120 \lambda_{i}$ in the high injection rate simulation domain at $90^{\circ}$ to the initial magnetic field after $64 \Omega_{i}^{-1}$. The density component of the interaction region is relatively extensive and the solar wind ions are significantly depleted over a large region. Injection is at $90^{\circ}$ to the initial magnetic field direction. 
completely of solar wind protons. This part of the interaction region is, again, not as large as the magnetic perturbations but still spans a region over $50 \lambda_{i}$ long. This interaction region is by far the largest of all the simulations performed.

\subsection{Mass Loading}

Due to mass loading of the injected ions upon the solar wind, conservation of momentum demands that as the injected ions are accelerated up to the solar wind velocity, so too must the solar wind ions be equivalently slowed. Since the simulation is performed in the solar wind frame where it will have zero velocity this will be seen as the solar wind being accelerated.

\subsubsection{Injection at $45^{\circ}$ to the Initial Magnetic Field}

As mass is loaded onto the solar wind from the beginning of the simulation, so too is the solar wind accelerated right away by the injected ions. Since the injection is at $45^{\circ}$ to both the $\hat{x}$ and $\hat{y}$ axes, the solar wind is primarily accelerated in these directions also as shown by Figure 5.21.

Initially the solar wind is accelerated away from the main ion cloud in $+\hat{x}$ along the $\hat{x}$ axis and in $\hat{y}$ along the $\hat{y}$ axis after approximately $40 \Omega_{i}^{-1}$ up to a velocity of $0.5 V_{A}$ in each direction. Whilst these directions of acceleration are initially mutually exclusive, the solar wind ions which were initially moving only in the $+\hat{x}$ direction are then further accelerated to be moving in $+\hat{y}$ and $+\hat{z}$. The Alfvén wings which are formed are also comprised of accelerated particles moving mostly in the $+\hat{y}$ direction at $0.25 V_{A}$ but they also have a smaller parallel velocity of $0.1 V_{A}$. In this simulation the solar wind is effectively swept up ahead of the main ion cloud until it is able to slip around the core on its field lines and thence eventually return to 'normal'.

\subsubsection{Injection at $0^{\circ}$ to the Initial Magnetic Field}

After the initial injection whilst the largest acceleration of the solar wind is in the $+\hat{x}$ direction to $0.4 V_{A}$, some of the solar wind protons are accelerated in the $-\hat{x}$ direction to $-0.2 V_{A}$. From the parting of the field lines the particles are also accelerated to move in both the $\pm \hat{y}$ direction at $0.3 V_{A}$. Due to the resultant gyration of the particles there is also motion in the $\hat{z}$ direction.

The solar wind is affected far less in this geometry than in the $45^{\circ}$ geometry as even far in to the simulation, due to the expansion of the cloud providing a larger 

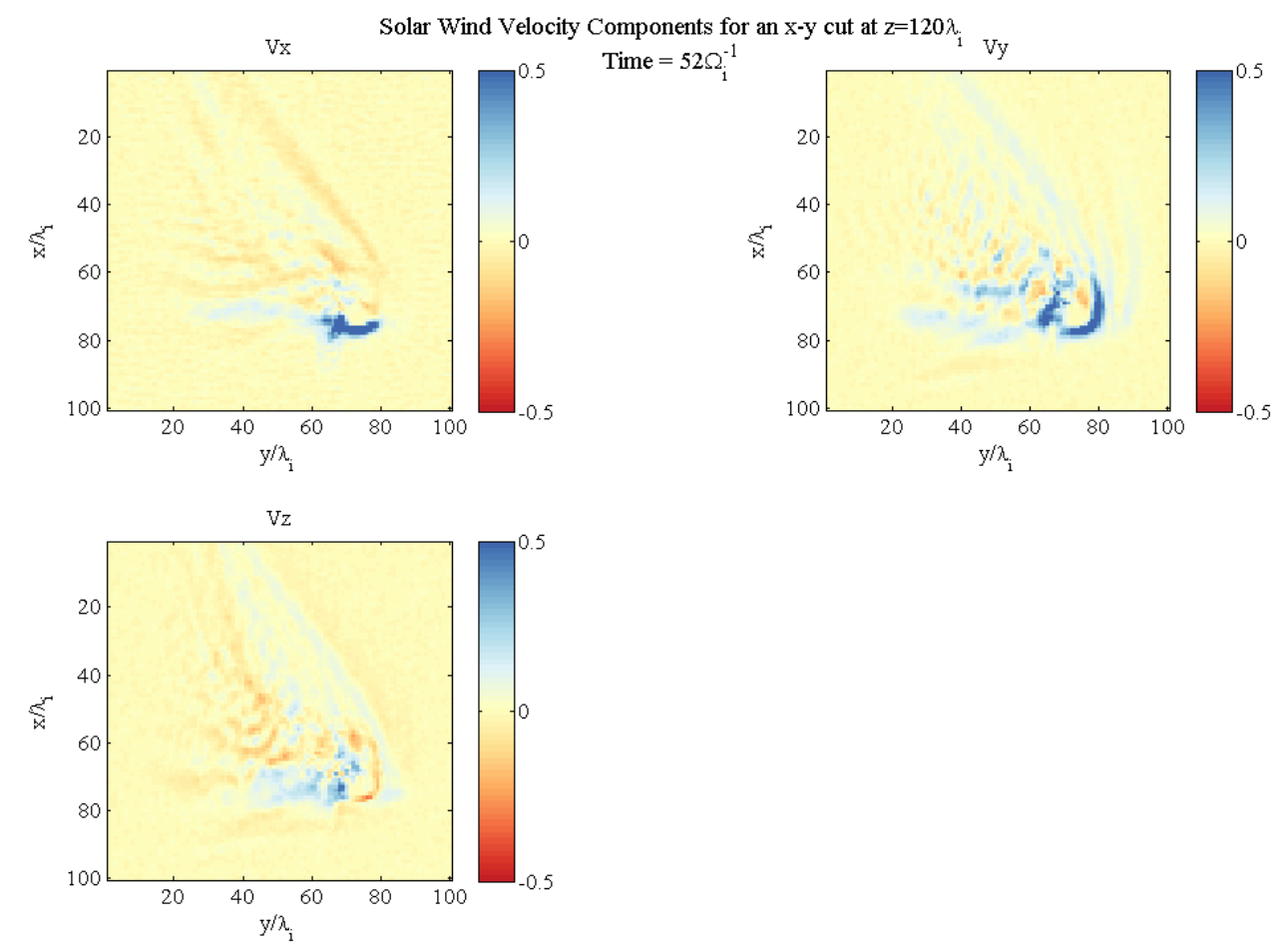

Figure 5.21.: The average velocity of the solar wind ions in each cell on an $\hat{x}-\hat{y}$ plane at $\hat{z}=120 \lambda_{i}$ in the $45^{\circ}$ simulation domain after $52 \Omega_{i}^{-1}$. The ions are primarily travelling in the $+\hat{x}$ and $+\hat{y}$ directions with some split along the $\hat{z}$ direction. This acceleration is a result of the mass loading process as the energy is transferred from the injected ions to the solar wind protons. 

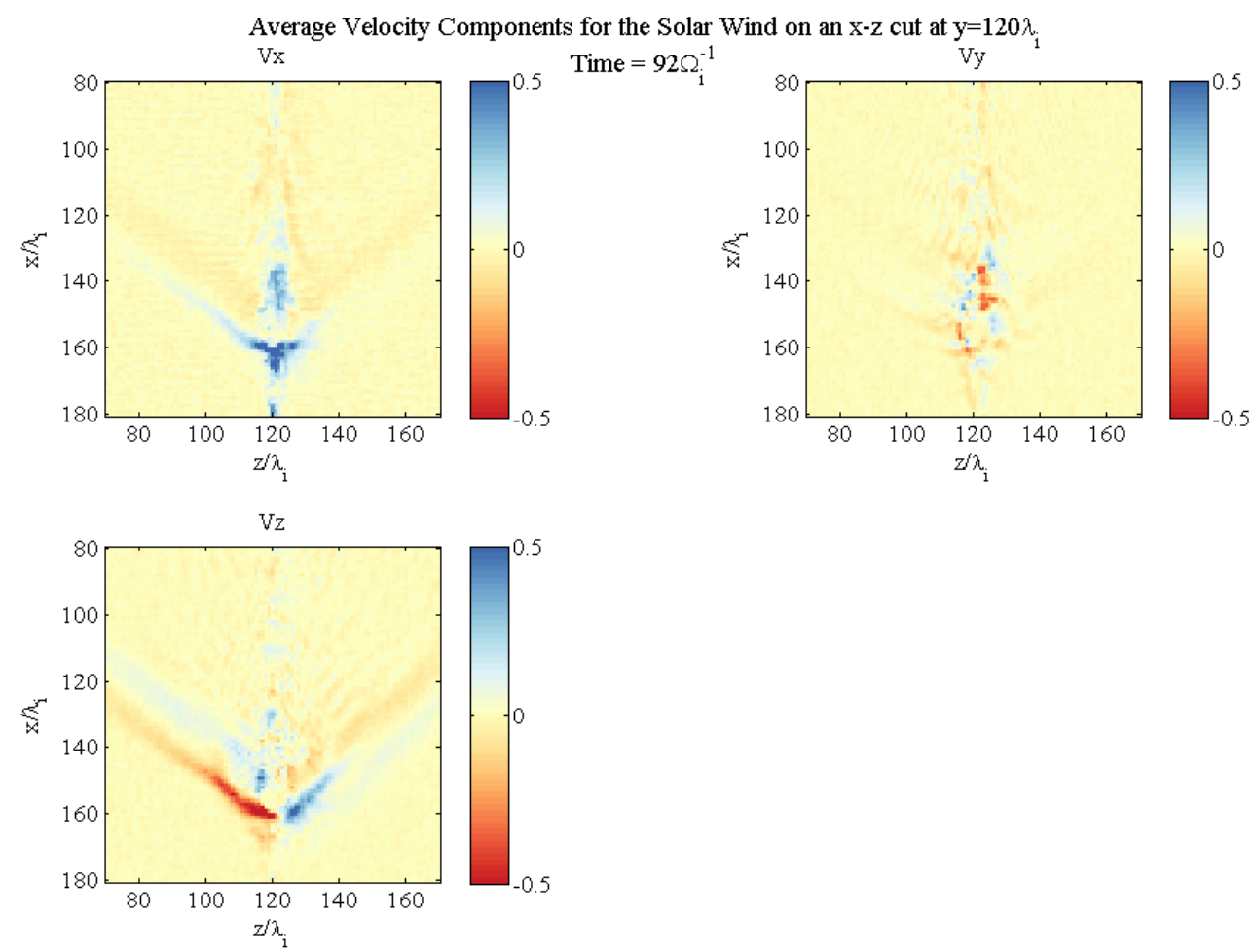

Figure 5.22.: The average velocity of the solar wind ions in each cell on an $\hat{x}-\hat{y}$ plane at $\hat{z}=120 \lambda_{i}$ in the $0^{\circ}$ simulation domain after $92 \Omega_{i}^{-1}$. The ions have the largest velocities in the $\hat{x}$ and $\hat{z}$ directions. This is due to being swept up by the ion cloud as a part of the mass loading process and being accelerated perpendicular to the field as the field lines are curved. This results in more coherent gyration of the solar wind protons in these regions. 
area of influence in which it can affect the solar wind, it has only been accelerated in the $+\hat{x}$ direction by $0.4 V_{A}$ as can be seen in Figure 5.22. The main evolution over the simulation is the size of the accelerated area. Whilst the solar wind is eventually also moving in other directions, this is largely due to gyration effects.

\subsubsection{Low Injection Rate at $90^{\circ}$ to the Initial Magnetic Field}

With the injection being at $90^{\circ}$ once more, the main acceleration of the solar wind is in the $+\hat{y}$ direction along with the injected ions. As the injection rate is low enough to not significantly disturb the solar wind the ions accelerated in $+\hat{y}$ are then left behind the spacecraft, gyrating around their field lines. In addition to the acceleration in $\hat{y}$ to $0.3-0.4 V_{A}$ there is also a parallel component to the acceleration such that some of the solar wind ions move along the field at $\pm 0.2 V_{A}$. Due to the levels of mass being injected being low the effects of mass loading in this simulation are relatively minor.

\subsubsection{High Injection Rate at $90^{\circ}$ to the Initial Magnetic Field}

As the effects in the low injection rate simulation are weak, so are the effects in this simulation correspondingly strong. The solar wind ions are, within $4-5 \Omega_{i}^{-1}$, accelerated by $0.5 V_{A}$ in both $+\hat{y}$ and $\pm \hat{x}$. As the mass loading continues the solar wind is accelerated further to $0.7 V_{A}$ in the $+\hat{y}$ direction as well as there being more extensive regions where the ions are accelerated along $\pm \hat{x}$ to $0.5 V_{A}$ near the leading edge where the mass is being actively loaded and the velocity slows to $0.25 V_{A}$ further back where the mass was first loaded. Figure 5.24 shows the larger acceleration effects of the mass loading on the solar wind ions as they reach far higher speeds and a much larger simulation volume is affected.

In addition to this there is also significant acceleration in the $\hat{z}$ axis up to maximums of $0.6 V_{A}$. Ultimately the mass loading effect is so strong in this simulation that the solar wind is 'blasted' from the path of the injected ion cloud although it is not quite so high as to cause the formation of a shock. 

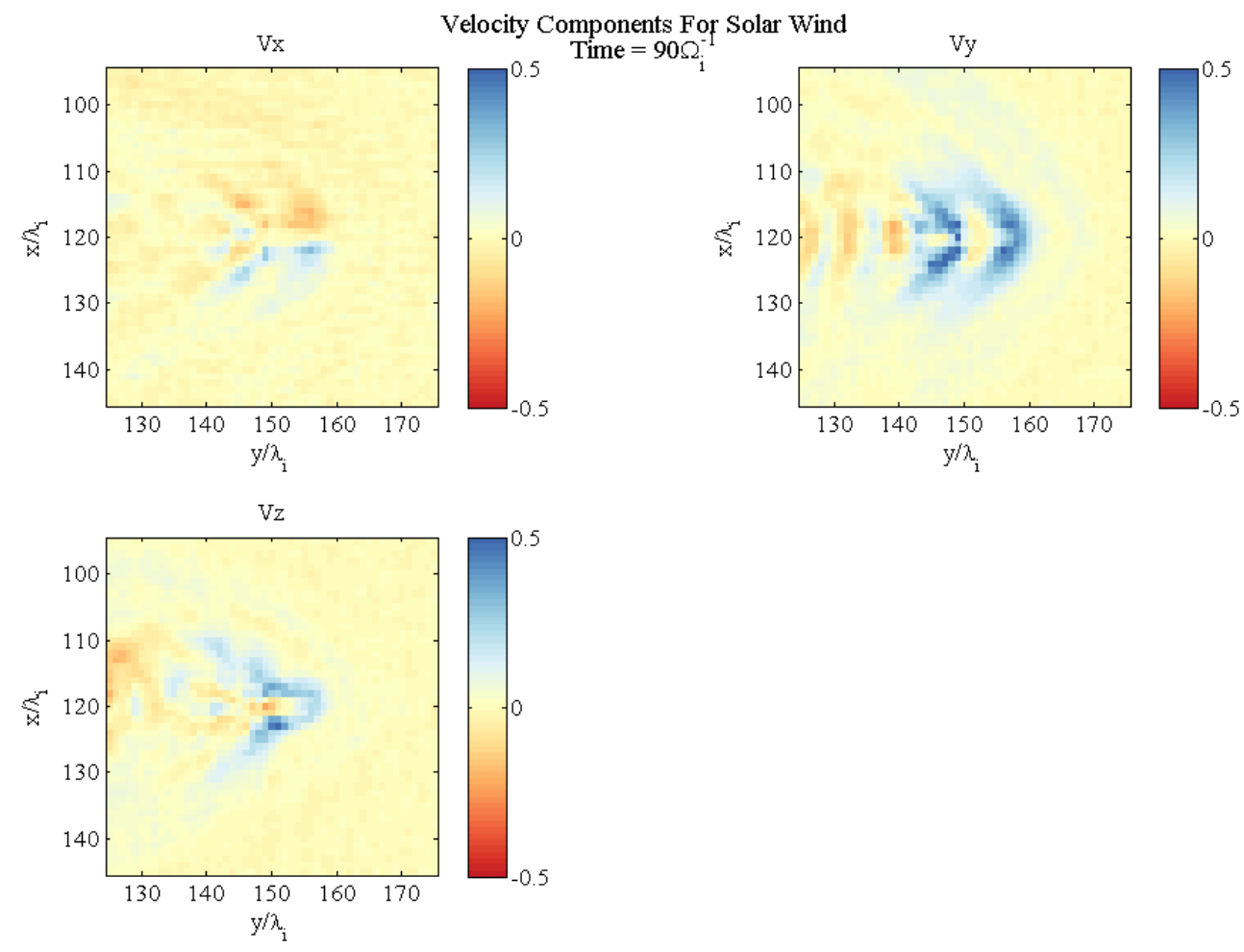

Figure 5.23.: The average velocity of the solar wind ions in each cell on an $\hat{x}-\hat{y}$ plane at $\hat{z}=120 \lambda_{i}$ in the low injection rate simulation domain at $90^{\circ}$ to the initial magnetic field after $90 \Omega_{i}^{-1}$. The solar wind has been accelerated in the direction of the pickup ions but the effect is far less than for the greater injection rates as the ions have a directed velocity of the same order as their thermal speed. 

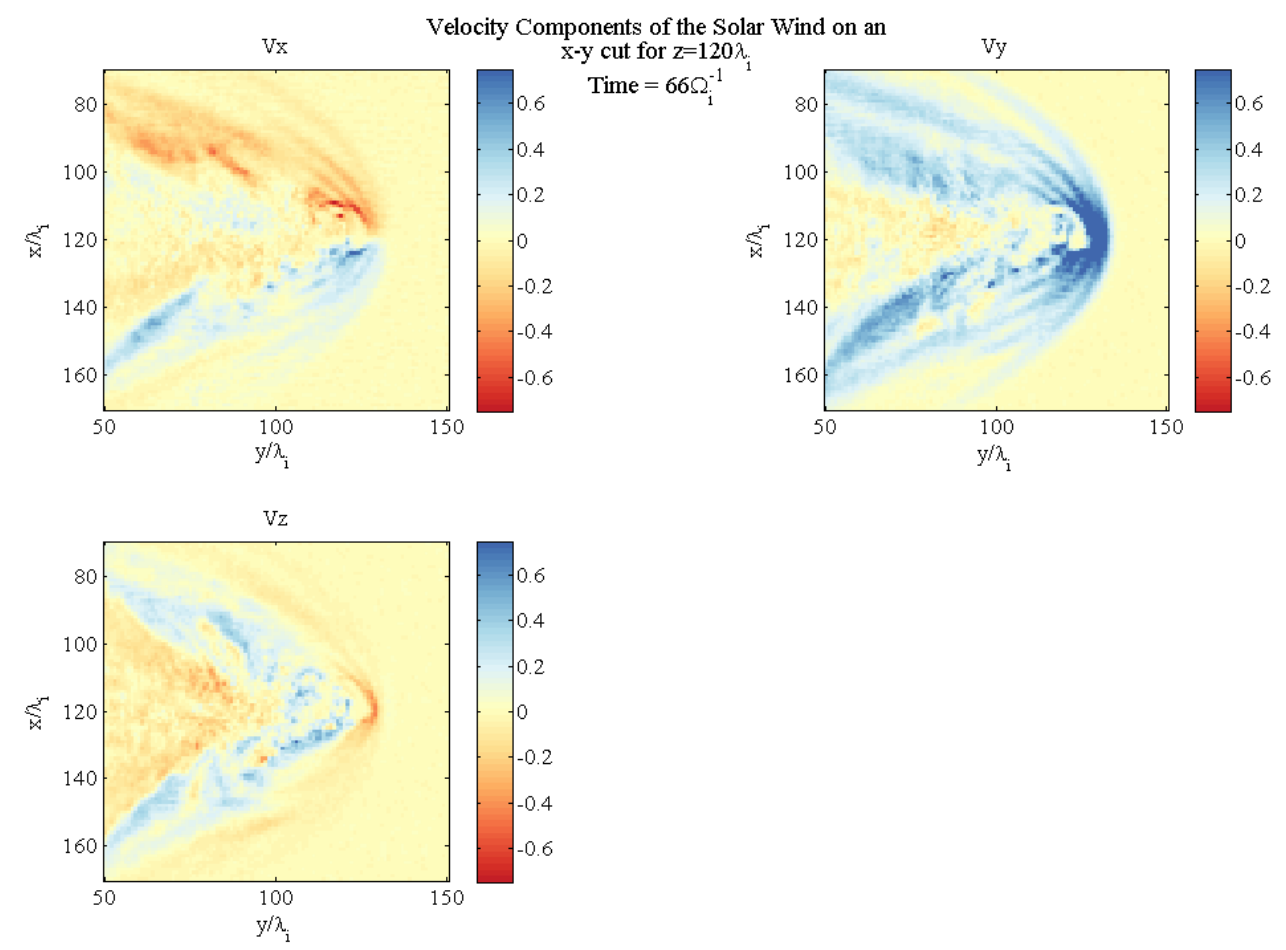

Figure 5.24.: The average velocity of the solar wind ions in each cell on an $\hat{x}-\hat{y}$ plane at $\hat{z}=120 \lambda_{i}$ in the high injection rate simulation domain at $90^{\circ}$ to the initial magnetic field after $66 \Omega_{i}^{-1}$. The region of the simulation affected by the mass loading is far greater in this case as can be seen by the much larger velocities of the solar wind ions. 


\section{Interaction of Spacecraft Thrusters with the Solar Wind - Particle Trajectories}

The following chapter discusses individual particle kinetics and investigates some characteristic particle trajectories for each of the simulations performed. Trajectories of interest are both those of 'standard' pickup ions, that is, those ions which are rapidly caught up on magnetic field lines and are, in the simulation frame, decelerated to the solar wind speed and then merely perform gyromotion (as described in more detail in Chapter 2) as well as, perhaps more importantly, those ions whose trajectories depart from this simpler behaviour in some way. The aim of this work has been to look for signs of particle scattering, energisation and diffusion as signatures of instabilities which might have been expected were not observed. It has, however, proven somewhat difficult to unravel all of the details of particle kinetics due to the evolution over time and space and therefore we only present introductory work here. Particles were selected for closer inspection due to their rapid loss of energy and negligible scatter, in the case of injected ions, as representative of a standard pickup ion trajectory. Particles were also selected if some component of their velocity was significantly different than was expected of a standard pickup ion, e.g., if they achieved very high velocities in some direction due to wave interactions or if they were scattered significantly either through interactions with Alfvén waves or through interactions with the bow waves formed. Throughout this chapter all particle trajectories, their properties and ambient field data are given in the solar wind frame in which all of the simulations were performed.

\subsection{Reference Simulation}

The reference simulation is the simulation with the standard parameters from which the other simulations will vary. The parameters for this simulation were given 

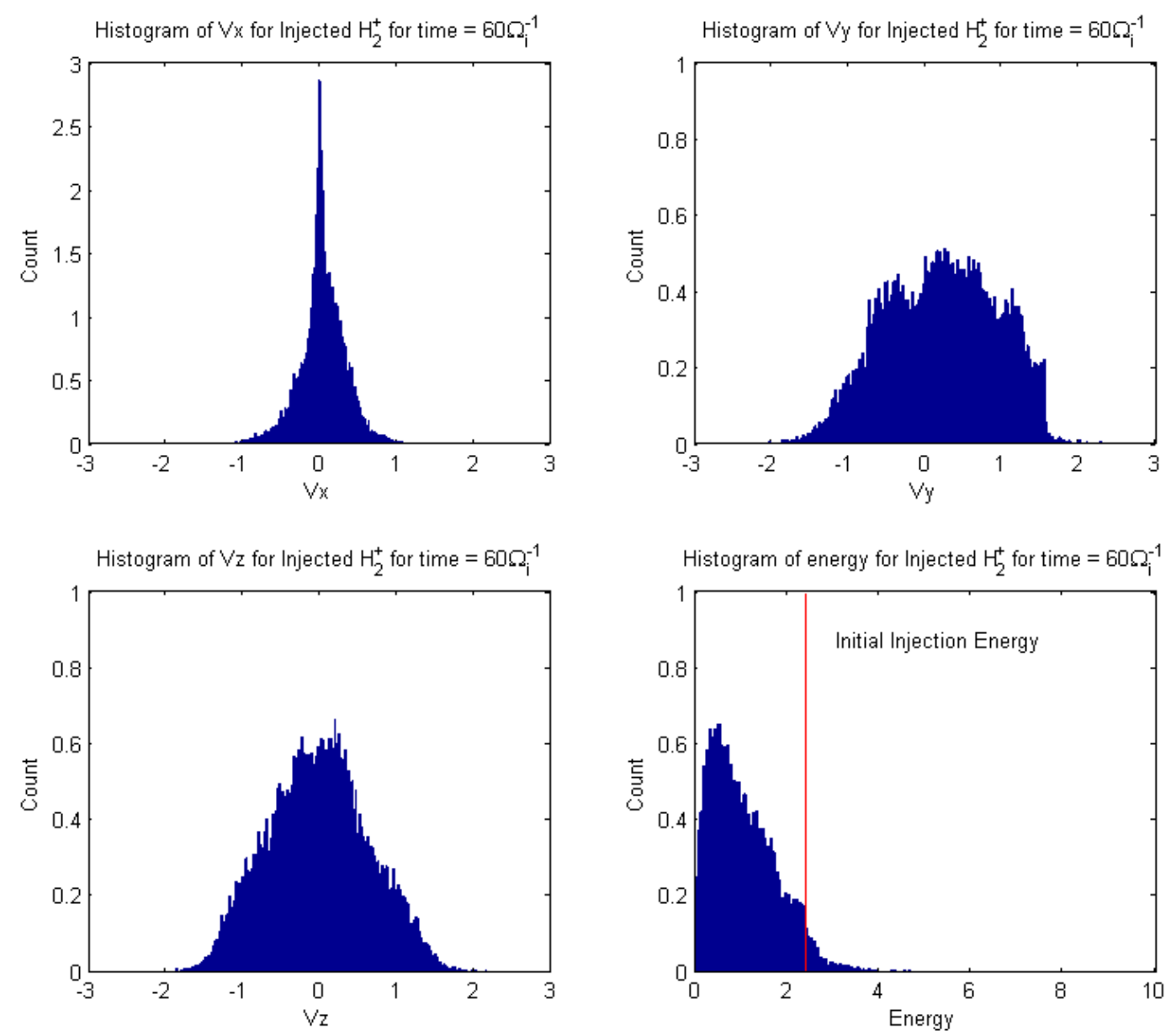

Figure 6.1.: A normalised histogram taken after $60 \Omega_{i}^{-1}$ of the spread of the components of the injected hydrogen's velocity and resulting overall energy in the reference simulation. The red line on the energy plot shows where a newly injected particle's energy lies.

previously in table 4.1 in Chapter 4 and are restated here. They include solar wind conditions which might be found at ten solar radii and approximately $30 \mathrm{~g}$ of ions injected over the simulation duration directed at $90^{\circ}$ to the ambient magnetic field. The domain size is a cube of sides $240 \lambda_{i}$ and a duration of $120 \Omega_{i}^{-1}$, the newborn particles are injected with a velocity of $1.5 V_{A}$ and the magnetic field strength is assumed to be $500 \mathrm{nT}$ and the solar wind number density to be $10^{9} \mathrm{~m}^{-3}$.

Figure 6.1 shows a histogram of the injected hydrogen's velocity components. From this figure it can be seen that the injected ions are pitch angle scattered rapidly as distributions of this form exist from as little as $20 \Omega_{i}^{-1}$ into the simulation, albeit less well defined. The ions are evenly pitch angle scattered in the parallel 
direction due to the injection angle being $90^{\circ}$ to the ambient magnetic field and therefore the scattering effect from both the forward and backward parallel propagating Alfvén waves is the same. Whilst most of the particles lose energy to waves and the acceleration of solar wind ions it can be seen that some few do gain energy beyond their initial injection energy. The velocity distribution of the injected hydrogen ions for this simulation is initially flat in the perpendicular direction but over time it evolves towards a peak centred on zero and then broadens as the effects of pitch angle scattering occur. The peak in the parallel velocity is initially very sharp and centred on zero as the ions initially have no component of their injection velocity directed along the $\hat{x}$ axis. Over the duration of the simulation this sharp peak broadens and spreads to higher parallel velocities with a slight preference to scatter into the $-\hat{x}$ direction approximately $5-10 \Omega_{i}^{-1}$ earlier than into the $+\hat{x}$ direction. The energy distribution is initially sharply peaked at the injection energy but evolves towards a cold Maxwellian distribution over the simulation's duration.

Figure 6.2 shows the velocity distribution of the hydrogen ions with the red lines being the paths particles would diffuse along into the bispherical shell distribution. It should be noted that for this figure, and all other bispherical distribution figures, only every $n^{\text {th }}$ particle was used where the value of $\mathrm{n}$ was varied in order to include enough particles to properly populate the figures and obtain a representative distribution but also few enough that it remained as uncluttered as possible. Energetically speaking, the paths indicated for the bispherical distributions represent contours of constant energy in the wave frame and particles are scattered along them by both forward and backward propagating Alfvén waves, hence the two spherical distributions centred on $\pm V_{A}$. It can be seen that this is in fact the case as there is a larger concentration of ions along those lines which have been scattered by forward and backward propagating Alfvén waves in a roughly equal ratio. Those particles centred with a zero parallel velocity have interacted equally with both direction waves with a net effect, in terms of pitch angle scattering, close to zero due to the injection angle being $90^{\circ}$. The particles mostly diffuse to lower perpendicular velocities due to the fact that they must, on average, lose energy in this frame and the upper paths of the bispherical distribution correspond to increasing energy. Energy diffusion could also be due to other effects such as oblique waves or nonlinear structures and strong field intensities, however, the bispherical paths bound most particles.

One of the most common trajectories that an injected $\mathrm{H}_{2}^{+}$ion follows shows a similar path to a test particle. All ions are injected initially with a velocity purely perpendicular to the ambient magnetic field with a magnitude of $1.5 V_{A}$. The typical ion is then caught on the local magnetic field line and begins to undergo gyromo- 


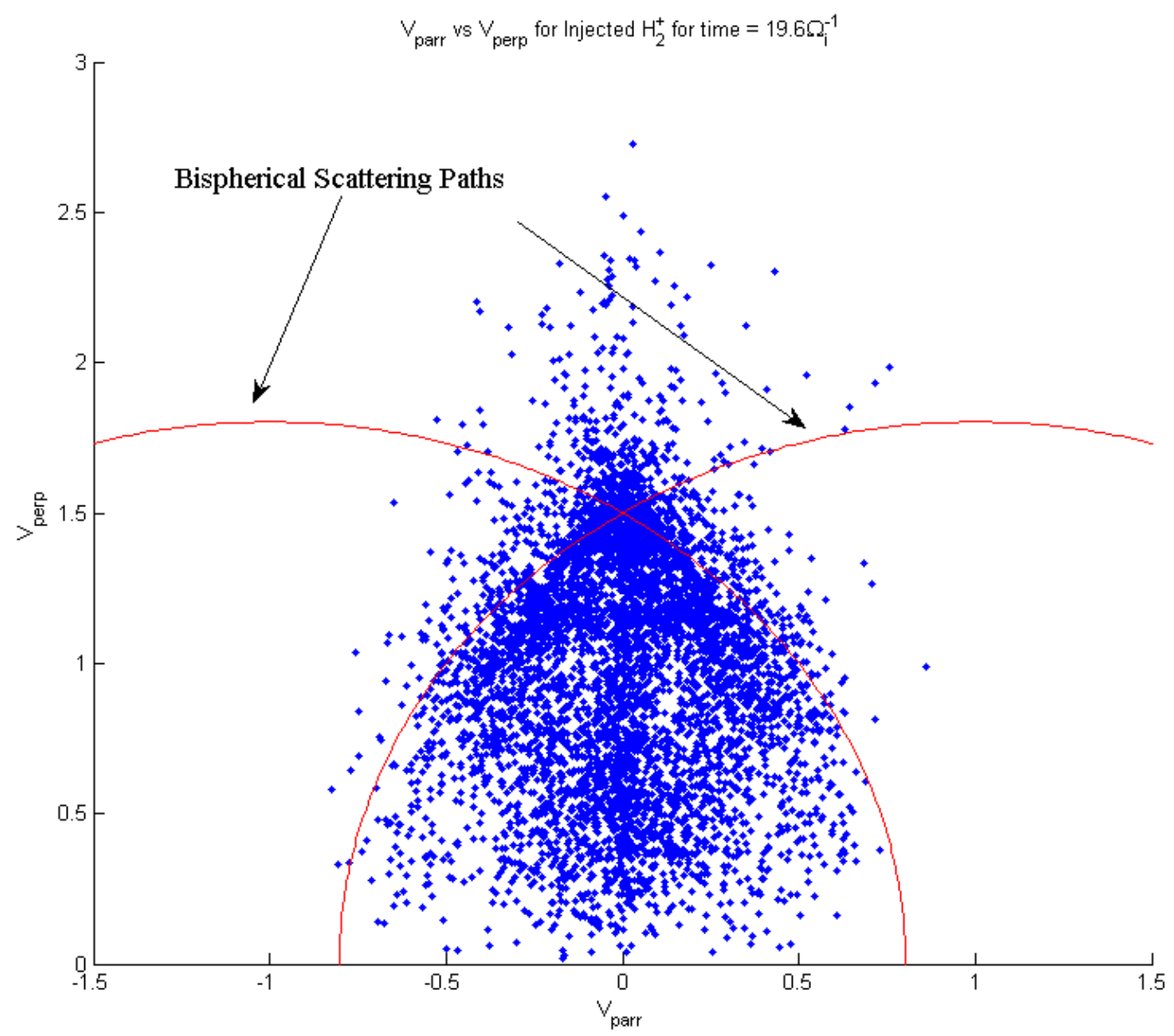

Figure 6.2.: A parallel-perpendicular velocity distribution of the injected hydrogen ions in the reference simulation after $19.6 \Omega_{i}^{-1}$. The red lines show the scattering paths of a bispherical shell distribution assuming parallel propagating Alfvén waves. 

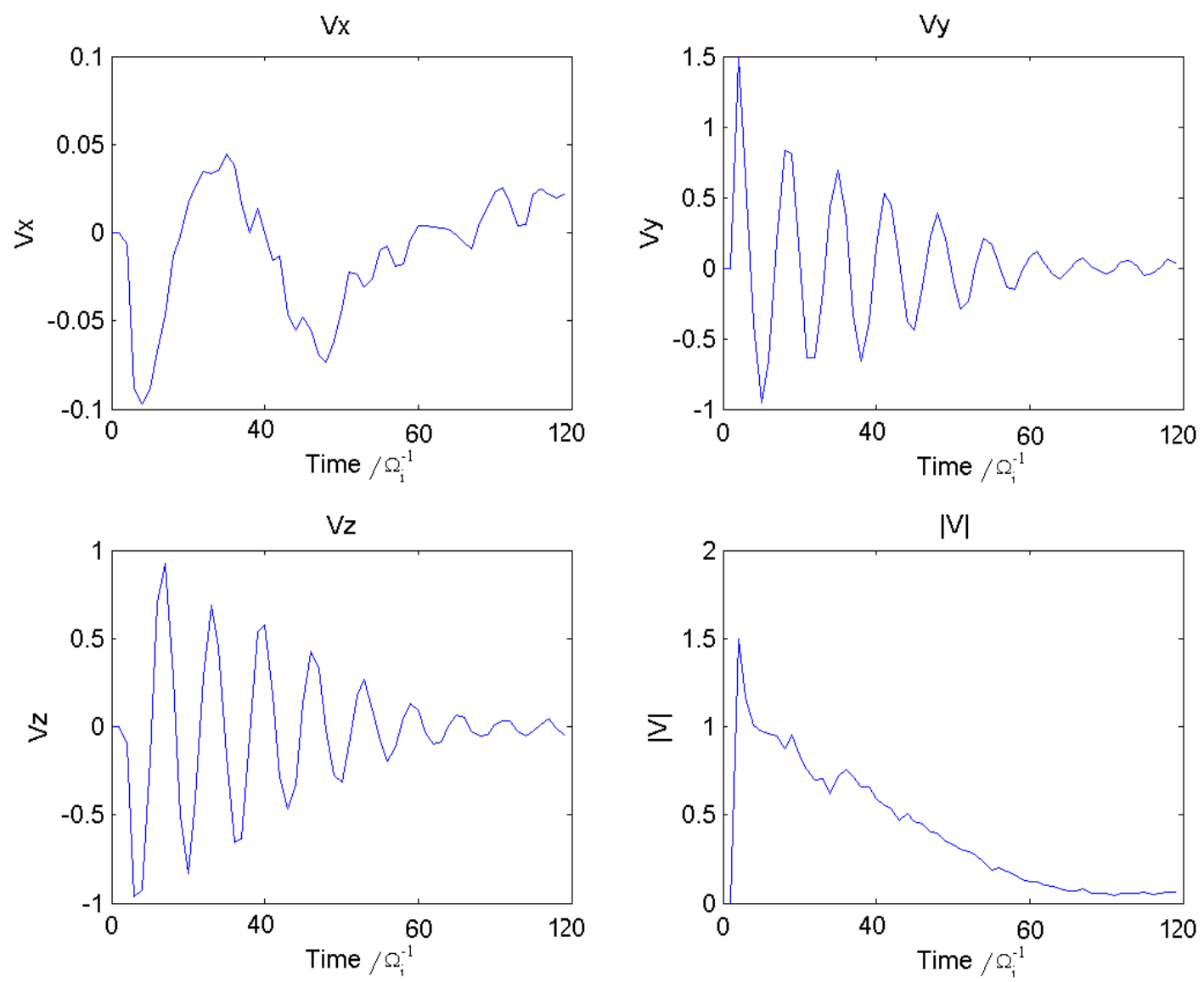

Figure 6.3.: Components and magnitude of an injected hydrogen's velocity in the solar wind frame. The particle performs cyclotron motion with an ever decreasing larmor radius as its free energy is used to drive waves.

tion. This motion causes fluctuations in both the magnetic and electric fields. The fluctuating fields, which also have contributions from other particles, act to scatter the ions in velocity space such that they will often attain some small parallel velocity. Additionally, in stimulating the wave activity the ions will usually lose energy overall and thus their perpendicular velocity will become lower.

There are other particles which interact with these fluctuations and over the duration of the simulation lose the majority of their kinetic energy to the fields. Such ions can go from having $|V|=1.5 V_{A}$ to having only $|V|=0.15 V_{A}$ over $80 \Omega_{i}^{-1}$. This is shown in Figure 6.3 which displays the components and magnitude of such a particle's velocity over the duration of the simulation, from this it can be seen that the particle is continuously losing energy as a result of driving the waves in the simulation. This can be clearly seen in the reduction of its perpendicular velocity as it decays throughout the simulation. These particles are fully picked up by the solar 

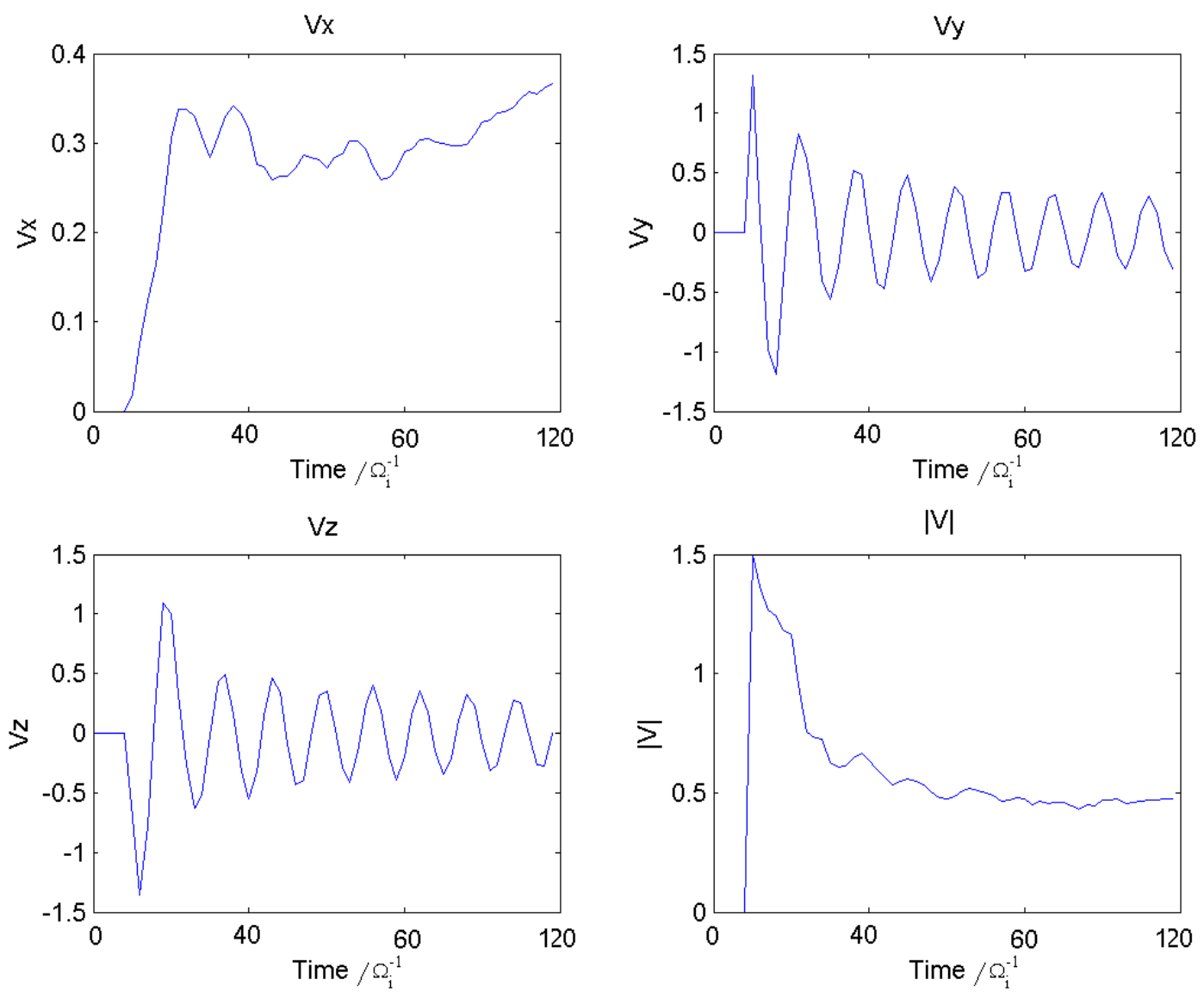

Figure 6.4.: Components and magnitude of an injected hydrogen's velocity in the solar wind frame. The particle is injected later into the simulation and can interact with the already-formed waves present in order to scatter and achieve some parallel acceleration at the expense of perpendicular energy.

wind and end up with similar velocities to the undisturbed background solar wind of $\approx 0.1-0.2 V_{A}$. The ion in Figure 6.3 is injected into the simulation early on and moves very little from its starting position, it begins a short distance behind where the bow wave will form and interacts with it almost immediately as it forms. It is then left behind and does not move significantly in any direction over the duration of the simulation but continues to lose energy as it is picked up by the solar wind and provides a source of free energy for electromagnetic fluctuations.

Particles which are injected slightly later interact a little differently as the fluctuating fields have had time to become established. These particles can experience significant electric fields depending on the section of the bow wave encountered and its propagation direction relative to the ambient magnetic field as shown by Figure 
4.2. This has the effect of pitch angle scattering the ions, such that they end up with a parallel velocity of a similar magnitude to their perpendicular velocity as shown by Figure 6.4, the pitch angle scattered ion encounters the bow wave shortly after being injected and is then scattered over approximately $10 \Omega_{i}^{-1}$. As it then travels in the $+\hat{x}$ direction away from the interaction region which forms a part of the hydrogen tail which is left behind. These ions have a reduced kinetic energy and also continue on to describe a helical trajectory.

Not all injected particles contribute to wave growth however as there are some which are in fact accelerated overall. Such particles do generally stimulate some wave growth at first as they lose some energy during early interactions which will have, either directly, stimulated waves or else will have energised solar wind ions which in turn generates wave activity. They can then interact with the higher amplitude waves and overall will end the simulation with a higher energy than they were injected with. Such energy gains are not so common nor so dramatic as those particles which lose energy and contribute to wave growth as was evidenced in Chapter 5 by the fact that large amplitude waves are generated which therefore means a diffusion of particles to lower energies must occur. Particles of this class which experience a weak energisation will typically go from $|V|=1.5 V_{A}$ to $1.8 V_{A}$ without moving significantly from their injection location and have therefore been picked up easily by the solar wind. Some other particles are, to a large extent, unaffected by the fluctuating fields. These particles are slightly scattered in velocity space and consequently lose a small amount of energy. However, in a slight contrast to the other particle classes discussed these ions are not significantly affected and will merely undergo gyromotion as they are picked up.

The nitrogen ions, being significantly heavier than the hydrogen ions, are not affected so strongly by the ambient fields. Typically these particles will experience a weak scattering effect due to interactions with the electric field but will generally continue on a trajectory very similar to that of a test particle and undergo cyclotron motion. Thus, even though the $\vec{E}$ field magnitude can become very large, the greater mass of these ions renders them, on the whole, largely unaffected over the duration they experience the fields as the solar wind requires timescales longer than the simulation duration to fully assimilate these ions. The fact the nitrogen ions are mostly unaffected is demonstrated in Figure 6.5 as the nitrogen ion is only scattered very slightly in the parallel direction and its velocity perpendicular to the magnetic field remains largely the same. These nitrogen ions will typically gyrate into the $-\hat{z}$ direction after being injected, forming part of the nitrogen 'clumps' mentioned in Chapter 4 . There are nitrogen ions which are scattered to slightly 

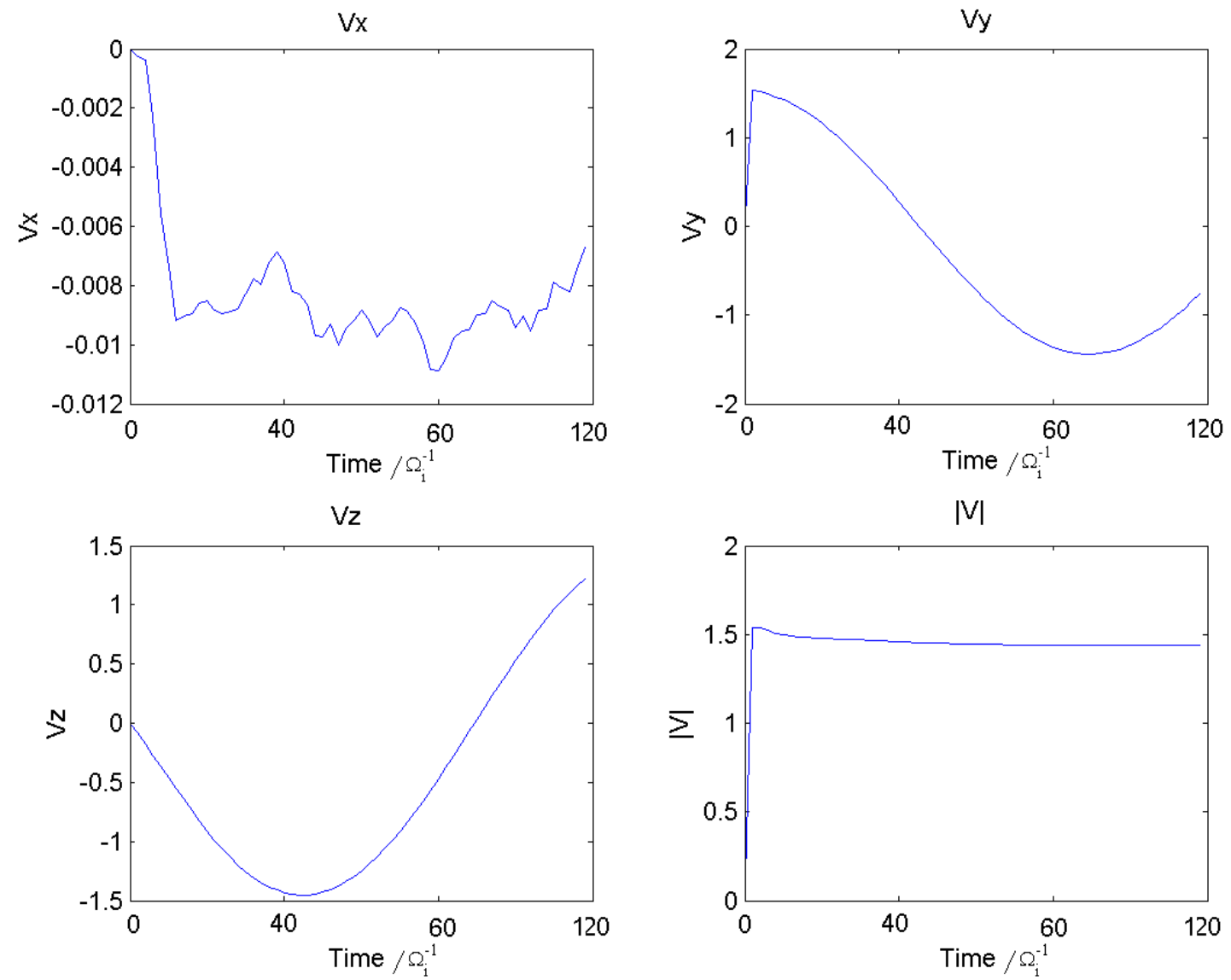

Figure 6.5.: Components and magnitude of an injected nitrogen's velocity. There is a very minor pitch angle scattering in the parallel direction as it gains a very small, negative $V_{x}$ whilst its perpendicular velocity remains largely unaffected by its interaction with the ambient solar wind plasma. 
larger degrees and do obtain a more significant parallel velocity. These ions do begin to perform cycloidal motion but due to the length of the simulation in comparison to the nitrogen gyroperiod (which are of a similar duration) it does not experience a significant scattering effect and thus does not travel a large distance along the $\hat{x}$ axis.

Solar wind particles experience the accelerating effects of mass loading and as the lightest ions in the simulation they respond quite rapidly to fluctuations in ambient conditions. The solar wind velocity distribution is initially isotropic and thus it is common to find particles describing a helix or cyclotron motion. Such particles can, with the right initial conditions, move towards the newly formed bow wave and encounter the interaction region from the outside. This results in the ion being swept up by the bow wave and accelerated along with it. It is by this series of events that particles are accelerated significantly, some having their energies increased by an order of magnitude or more. These trajectories are helical initially until the ion encounters the bow wave at which time it is deflected from its path very significantly as it moves along the outer edge of the interaction region as it is unable to penetrate the magnetic barrier along with other solar wind ions which have been accelerated away from their initial location where the interaction region formed and hence contributing to the strength of the bow wave as well as to the deficiency of solar wind protons within the interaction region.

There are also other particles which are accelerated but have different trajectories after this acceleration. They, too, begin with helical motion and encounter the bow wave. These ions, however, are not swept up in the same way and in fact reflect from the bow wave as they are accelerated and then reverse their $V_{x}$ whilst having a much increased perpendicular velocity. These particles, again, have their energies increased by an order of magnitude or more. Figure 6.6 shows such a solar wind particle as it encounters the bow wave and interaction region and is significantly accelerated as the electric field preceding it causes an acceleration along the $\hat{x}$ axis and also causes fluctuations of a smaller amplitude in the magnetic field for a duration of $40 \Omega_{i}^{-1}$.

\subsection{Low Injection Rate Simulation}

The ions are once again injected with a purely perpendicular velocity and therefore the typical trajectory of such a particle involves it becoming caught on the local magnetic field lines and performing cyclotron motion. Usually these injected par- 

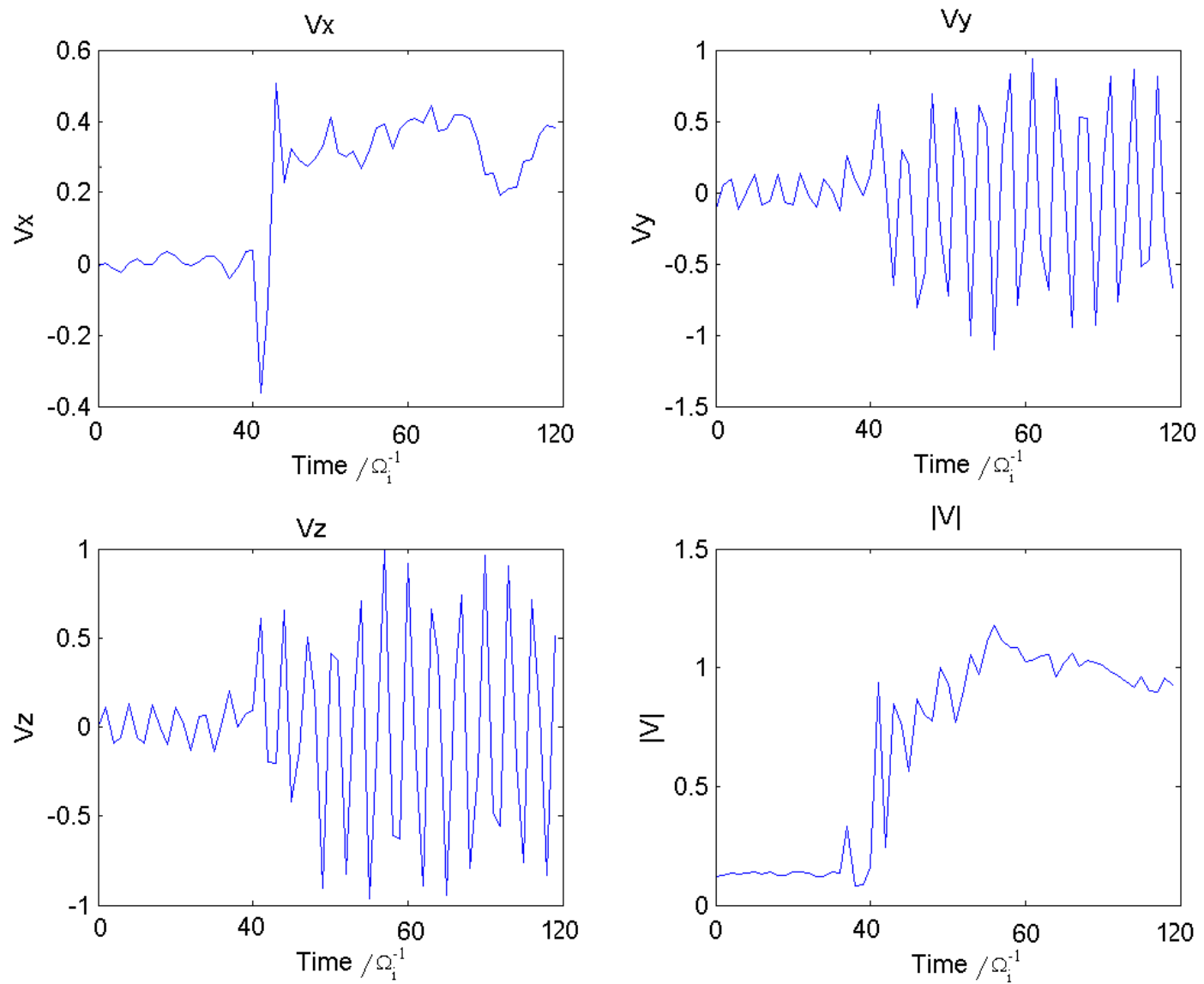

Figure 6.6.: Components and magnitude of a solar wind ion's velocity. The ion initially performs cyclotron motion until the bow wave and interaction region sweep over it at which point it experiences a relatively large acceleration and a reflection in its parallel velocity. 
ticles will lose energy overall as they cause fluctuations in the ambient electric and magnetic fields as part of their coupling with the solar wind protons. These particles, typically, will be scattered also by their interactions with the fluctuating fields and thus will often gain a small parallel velocity an order of magnitude lower than its gyrovelocity as the rate of pitch angle scattering is lower. There are also particles which retain the majority of their injection energy and only lose a very small fraction, such particles behave in a qualitatively similar manner to those that lose energy and the main difference is that they experience less pitch angle scattering and thus have a lower parallel velocity. Additionally, there are particles which are placed such that they can make a net gain in energy, these ions encounter electric fields parallel to the ambient magnetic field (i.e. the rest magnetic field, not the local magnetic field) and thus experience an acceleration and gain energy before encountering the scattering centres of the fluctuating fields which, for these particles, translates the parallel velocity into an additional perpendicular velocity.

Figure 6.7 shows a normalised histogram of the components of the velocity of the injected hydrogen ions. A comparison with Figure 6.1 shows a lower rate of pitch angle scattering as there is a smaller spread in parallel velocities although there is a larger, more even spread in perpendicular velocities where the distribution is not as peaked. Comparing the distribution of particle energies shows the largest difference, however, as the energy diffusion is not as far advanced. Due to fewer ions being injected, and hence fewer ions interacting with the solar wind plasma in order to generate waves which act as scattering centres, the average energy of a particle in this simulation is several times that of the reference simulation. As the simulation progresses, however, the distributions become more similar although the diffusion of the particles to lower energies remains slow. The perpendicular velocity distribution is initially peaked at $\pm V_{i n j}$ (the injection velocity) as fewer ions have zero perpendicular velocity. This shows that the rate of scattering in this simulation is lower than the reference and that it has a longer duration than the injected hydrogen gyroperiod. The distribution does then flatten in a similar manner to the reference as the simulation progresses until it has a very broad peak centred on $V_{\perp}=0$ at the end of the simulation although the final distribution remains flatter and less peaked than the reference simulation. The parallel velocity spreads and broadens over the duration, again with a preference to spread to $-\hat{x}$ before $+\hat{x}$. The energy of the ions also diffuses towards a Maxwellian in this case but it does so more slowly due to the lower scattering rate of the simulation and thus the resulting distribution is warmer at the end of the simulation.

Figure 6.8 shows that in this simulation the particles are also scattered into a 

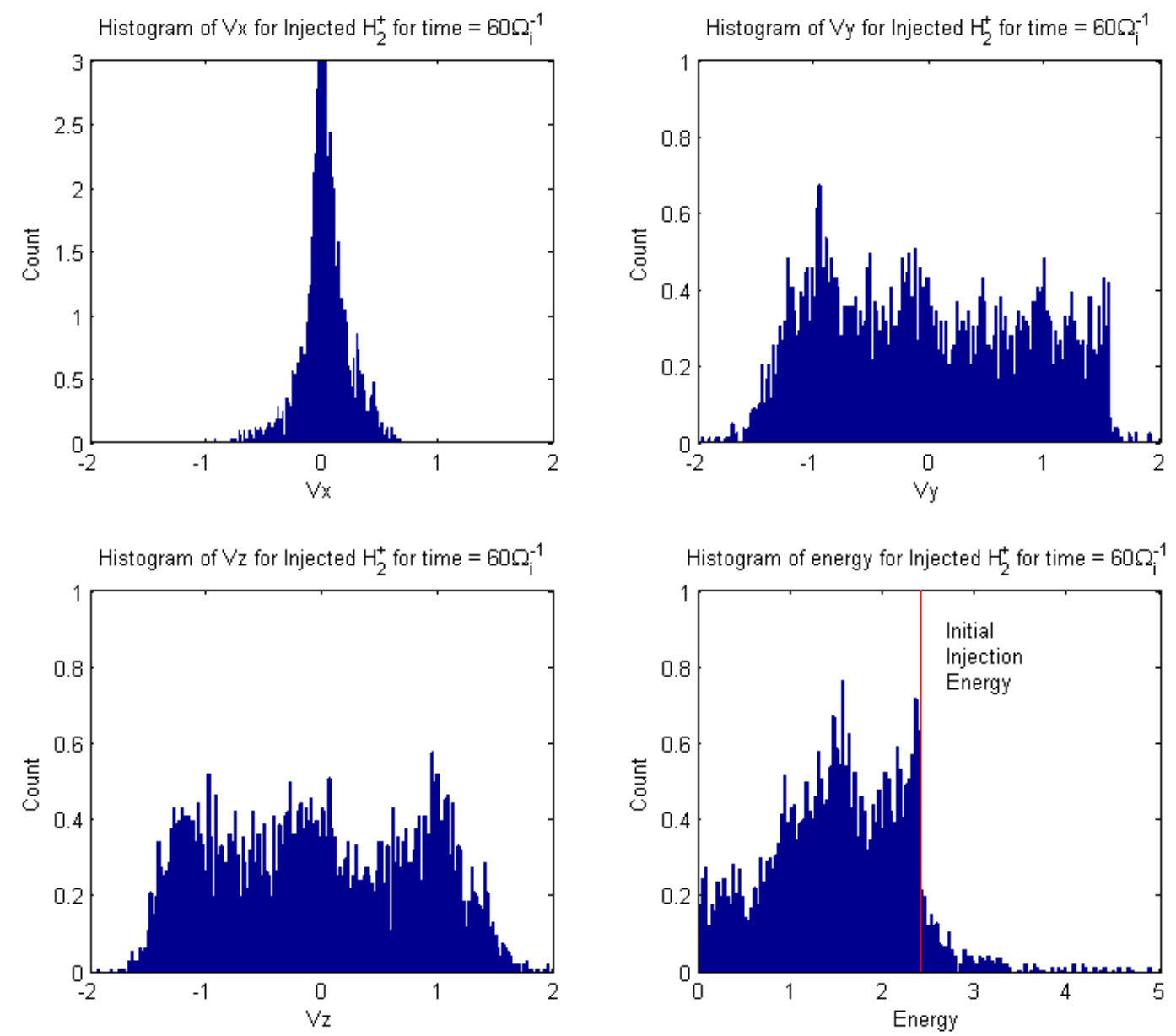

Figure 6.7.: A normalised histogram taken after $60 \Omega_{i}^{-1}$ of the spread of the components of the injected hydrogen's velocity and resulting overall energy in the low injection rate simulation. The red line on the energy plot shows where a newly injected particle's energy lies. 


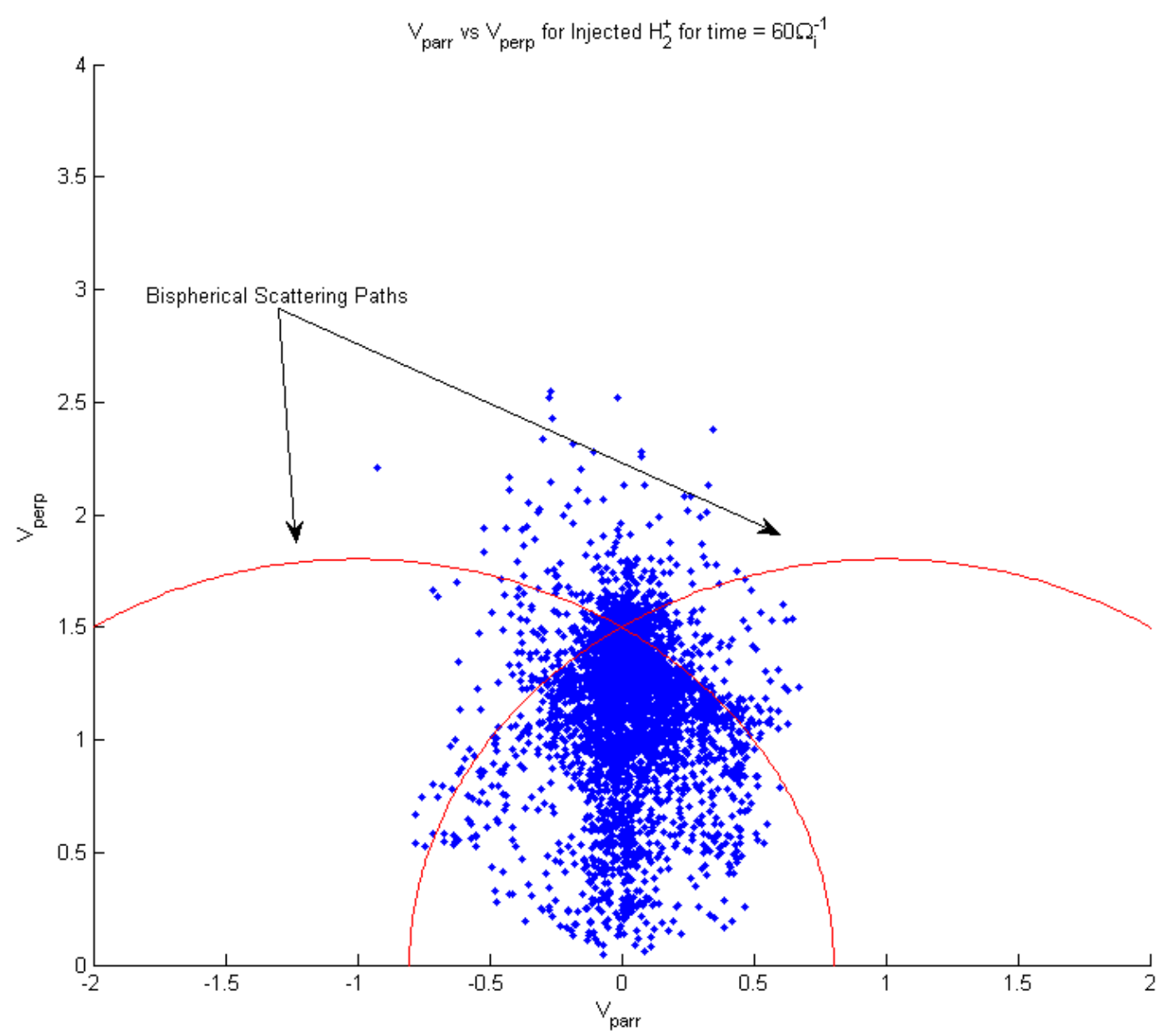

Figure 6.8.: A parallel-perpendicular velocity distribution of the injected hydrogen ions in the low injection rate simulation after $60 \Omega_{i}^{-1}$. The red lines show the scattering paths of a bispherical shell distribution assuming parallel propagating Alfvén waves.

bispherical distribution. The bispherical scattering paths bound the velocity distribution of the majority of the injected hydrogen ions in much the same way as in the reference simulation and thus the type of scattering the newborn ions experience does not appear to be changed with a reduced injection rate.

Again, particles which are injected into the simulation a little later can be scattered more significantly such that they can achieve relatively large parallel velocities. These particles usually lose energy overall during this scattering process and thus end up with a smaller gyroradius to their helical motion but other ions can retain the majority of their injected energy after being scattered in pitch angle. There are some particles which gain energy, these retain their perpendicular velocity and 
thus have been accelerated by a parallel electric field to parallel directed velocity components of the order of $0.5-1.0 V_{A}$ although there are, however, far less of these particles as the velocity distribution as a whole must diffuse to lower energies. Once more due to the nitrogen's much higher mass it remains almost unaffected by the perturbations it encounters. That effect is even more pronounced in this simulation as, with a lower injection rate, the perturbations are themselves smaller. Thus the $\mathrm{N}_{2}^{+}$ions display very little change and will typically just perform gyromotion around the local field lines. They begin to lose energy in a similar manner to the $\mathrm{H}_{2}^{+}$ions but over a larger time scale. The $\mathrm{N}_{2}^{+}$only displays 'pure' cyclotron motion as there are few, if any, which were found to differ from a textbook pickup ion trajectory, these ions gyrate into the $-\hat{z}$ direction over the duration of the simulation.

As with the reference simulation the mass loading effects influence the solar wind ions differently depending upon how and where the interaction occurs. As expected, the net effect is that the solar wind protons are energised in the simulation frame of reference. Most of these particles are accelerated by an encounter with the propagating bow wave, although in this case the strength of the bow wave is far less than in the reference simulation and it might, therefore, be expected to have a lesser effect on ions, this is not the case. The bow wave has sufficient strength to affect the individual solar wind protons to a similar degree as in other simulations, ions which encounter this bow wave can be accelerated to velocities very close to the ion injection velocity of $1.5 V_{A}$ extremely quickly, within $6 \Omega_{i}^{-1}$. The number of ions which are accelerated to this degree is, however, relatively small. Most of the solar wind ions affected are accelerated by a far more modest amount, typically by around $10 \%$ of their initial velocity although there are still a significant number which are accelerated to $0.5 V_{A}$ which is in keeping with the injected hydrogen being decelerated, in this frame, to approximately the same velocity.

Whilst the effect on individual ions remains comparable in a like for like comparison the number of ions affected in this case, with a lower injection rate, are consequently lower than the reference simulation due to the mass loading, and therefore free energy available, being proportionately less. Figure 6.6 shows a similar trajectory as a solar wind ion which drifts on a helical trajectory ahead of the bow wave and interacts with the bow wave at a later time and yet its velocity increase is comparable to the reference simulation and is not reduced due to the lower injection rate. 


\subsection{High Injection Rate Simulation}

The ions are injected with a velocity directed entirely perpendicular to the ambient magnetic field, however, the injection rate is far higher than in previous simulations and thus both the effects of mass loading and the free energy available is also much greater. A common trajectory for an injected hydrogen ion describes a decaying helix as the ion gyrates around the magnetic field lines and is often scattered so that it has a non-zero parallel velocity as shown in Figure 6.9. Throughout this motion the ions generally lose energy to wave generation and other interactions with the ambient plasma to a greater or lesser degree. The magnitude of energy loss depends mostly on the particle's phase with respect to the fluctuating fields as well as just the raw strength of those fields. Also due to the very high mass loading of the solar wind, injected ions tend to change energy over a relatively short amount of time, around $14 \Omega_{i}^{-1}$, and thus it can be said that the rate of diffusion of energy of the particles is higher. Thus there are particles which lose all of their additional energy and are thermalised in that very short timeframe in a similar manner to that shown by Figure 6.3. In this way the solar wind attempts to assimilate the injected ions although the loading of mass can become too high in localised regions for this to be completely effective and thus there is the formation of a powerful, extensive bow wave.

There are particles which interact with several wavefronts and it is possible for these ions to be affected differently by each wavefront. Some particles are decelerated by one wave only to be left with the correct velocity to be accelerated by a subsequent wave and then again decelerated by a following wave. In this manner the energy of certain particles can oscillate and it is likely that particles displaying such changes are trapped between two wavefronts and are being reflected between the two, gaining energy as a wave approaches it and losing energy as it approaches a wave. Figure 6.10 shows such a particle as it is slowed as it encounters the rear of a wavefront and accelerated as the following wavefront catches up to it resulting in an oscillating energy but with an increasing average.

Once more, particles injected later can experience a much greater effect from the scattering centres as they are injected within the interaction region. The result of this is that within a gyroperiod of the injected ion it can be significantly scattered in velocity space and can achieve parallel velocities of up to $1.0 V_{A}$ whilst maintaining an average speed perpendicular to the ambient field of $0.5 V_{A}$. It should be noted that these scattered particles initially lose energy and then display a similar oscillation of energy which is primarily indicated by its parallel motion and has an increasing 

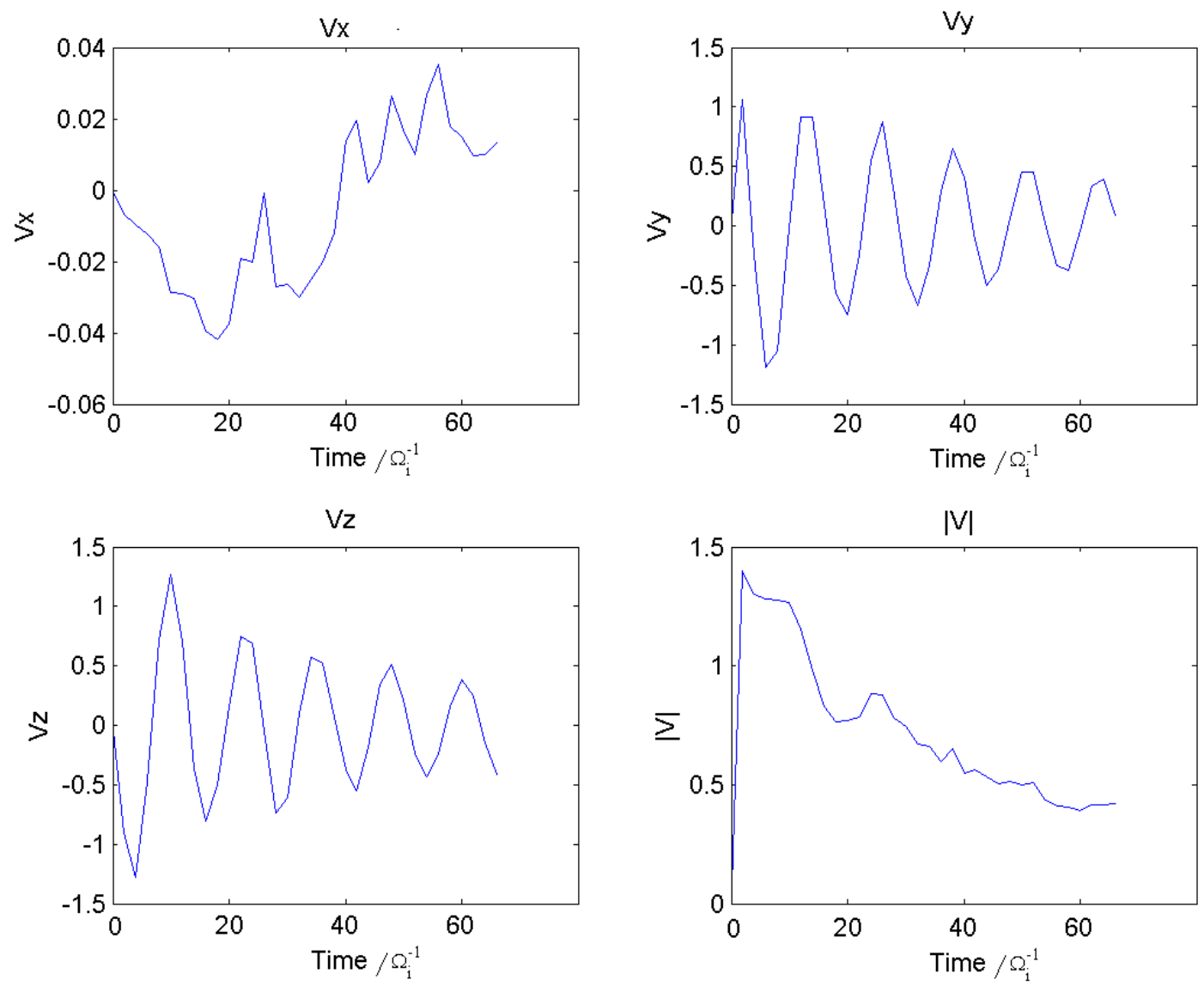

Figure 6.9.: Components and magnitude of an injected hydrogen's velocity. The decaying helix is the standard trajectory of an ion in this simulation as its energy is 'drained' as it stimulates wave growth. 

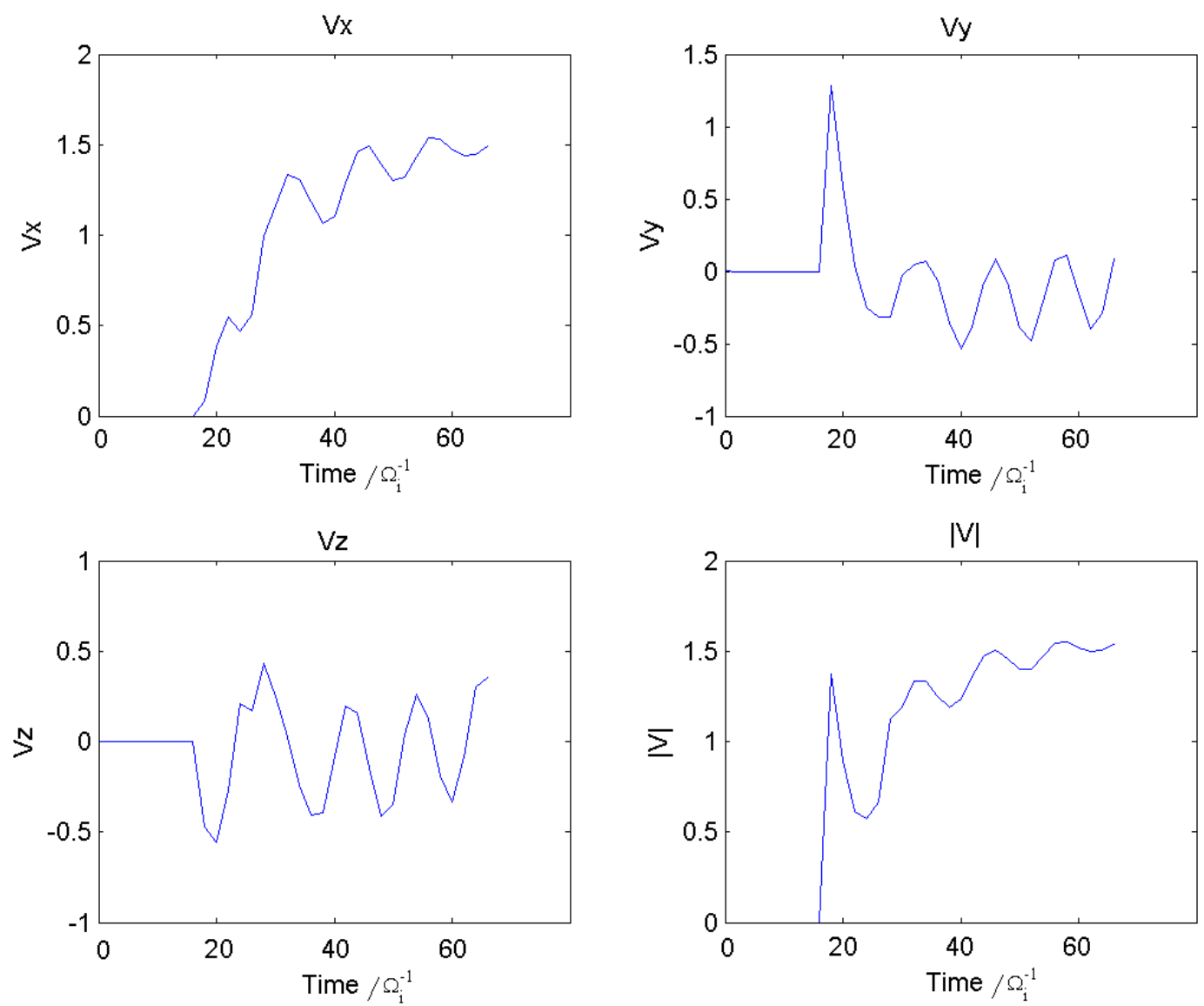

Figure 6.10.: Components and magnitude of an injected hydrogen's velocity. This ion is initially scattered strongly as it loses energy but then becomes trapped between wavefronts as it travels in the parallel direction which results in an energy increase on average. 
average over time. This effect can be more extreme if a particle is scattered such that the greater part of its velocity is in the parallel direction then the ion can gain energy more rapidly from reflection between the wavefronts.

Of course the contrary can also occur, there are particles which are reflected between the propagating wavefronts and gradually lose energy on average in a similar, but opposite, sense to that shown by Figure 6.10, this type of behaviour is not seen in the other simulations. Further, there are injected ions which are scattered and greatly accelerated as their parallel velocity is dramatically increased with no loss of perpendicular energy, this is due to enormous spikes in the local electric field induced as a result of the injection.

Figure 6.11 shows a normalised histogram of the components of the velocity of the injected hydrogen ions. Comparing this figure to Figure 6.1 shows that the rate of pitch angle scattering is higher in this simulation as the spread in the parallel velocity component is larger and less strongly peaked, this is also the case for both perpendicular components of velocity. The diffusion of energy is also more rapid with a higher injection rate, the general shape to the histogram is similar but is more smooth and also has a higher absolute range of energies which would be expected given the evidence of the acceleration of the injected ions. The perpendicular velocity flattens in this case almost before the ring velocity distribution has time to form properly but it retains a peak at the injection velocity. The distribution very rapidly forms the characteristic peak at $V_{\perp}=0$ seen in previous simulations which also becomes extremely broad in comparison to those simulations with a velocity range of $\pm 3 V_{A}$. Additionally the parallel velocity distribution becomes very broad also and thus this all indicates further that the scattering process at work is proportional to the strength of the injection and the number of injected ions present. The energy of the ions develops to a Maxwellian as in previous cases but with a relatively high energy tail with respect to other simulations.

Figure 6.12 shows that particles are largely scattering onto bispherical shells but that with an injection rate this high the assumption begins to break down as a larger fraction of the ion population is energised beyond that predicted by bispherical scattering although it remains a good approximation. It is thus possible that with an injection rate increased even beyond this that the bispherical distribution will cease to describe the velocity distribution of the injected ion population well.

The typical nitrogen trajectory again merely describes a helix, however, due to the much higher injection rate and the consequently higher field fluctuations, the motion of the $\mathrm{N}_{2}^{+}$is affected more in this simulation than in the previous simulations. Unfortunately due to the higher gyroperiod and mass of the nitrogen ions and the 

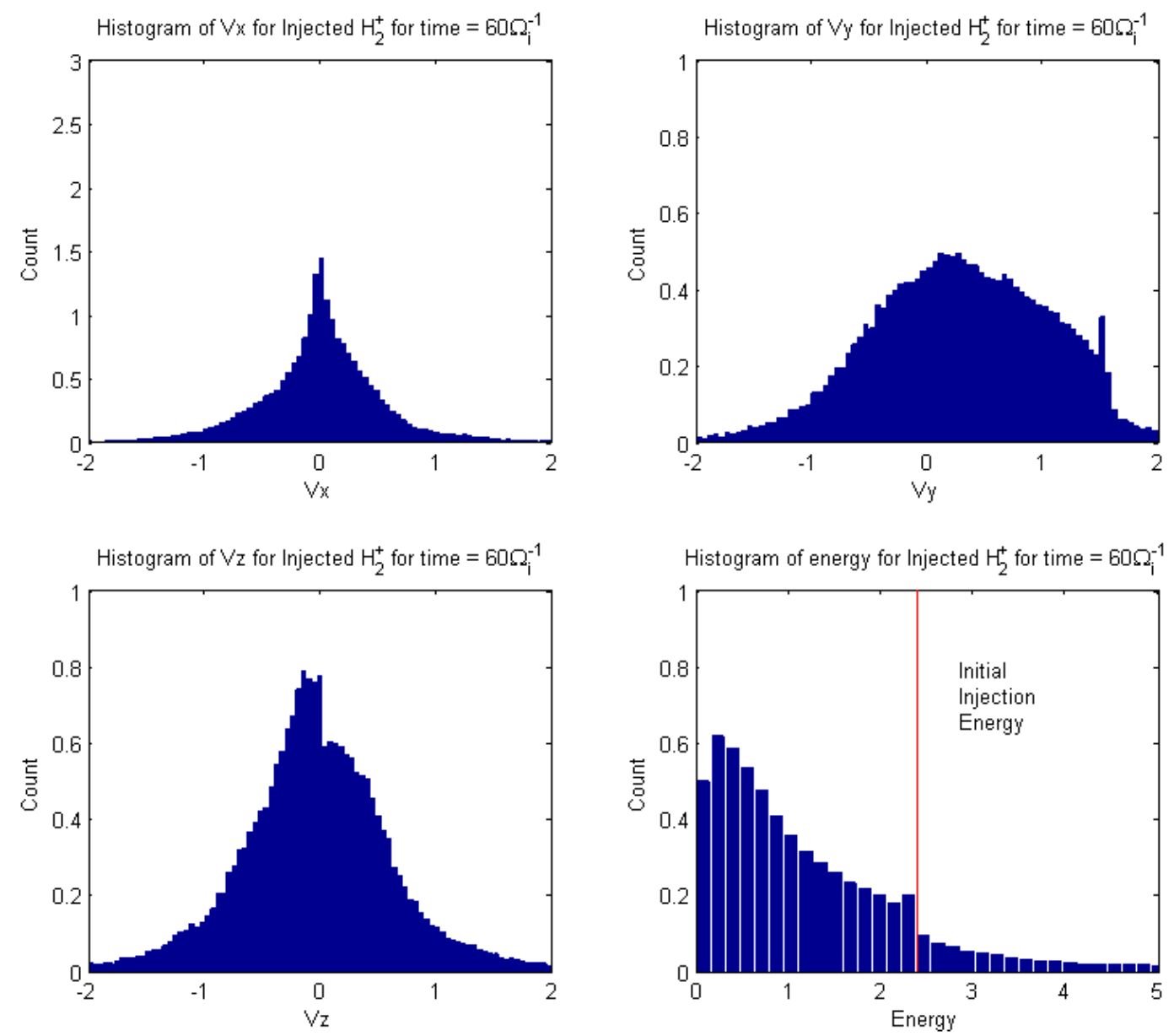

Figure 6.11.: A normalised histogram taken after $60 \Omega_{i}^{-1}$ of the spread of the components of the injected hydrogen's velocity and resulting overall energy in the high injection rate simulation. The red line on the energy plot shows where a newly injected particle's energy lies. 


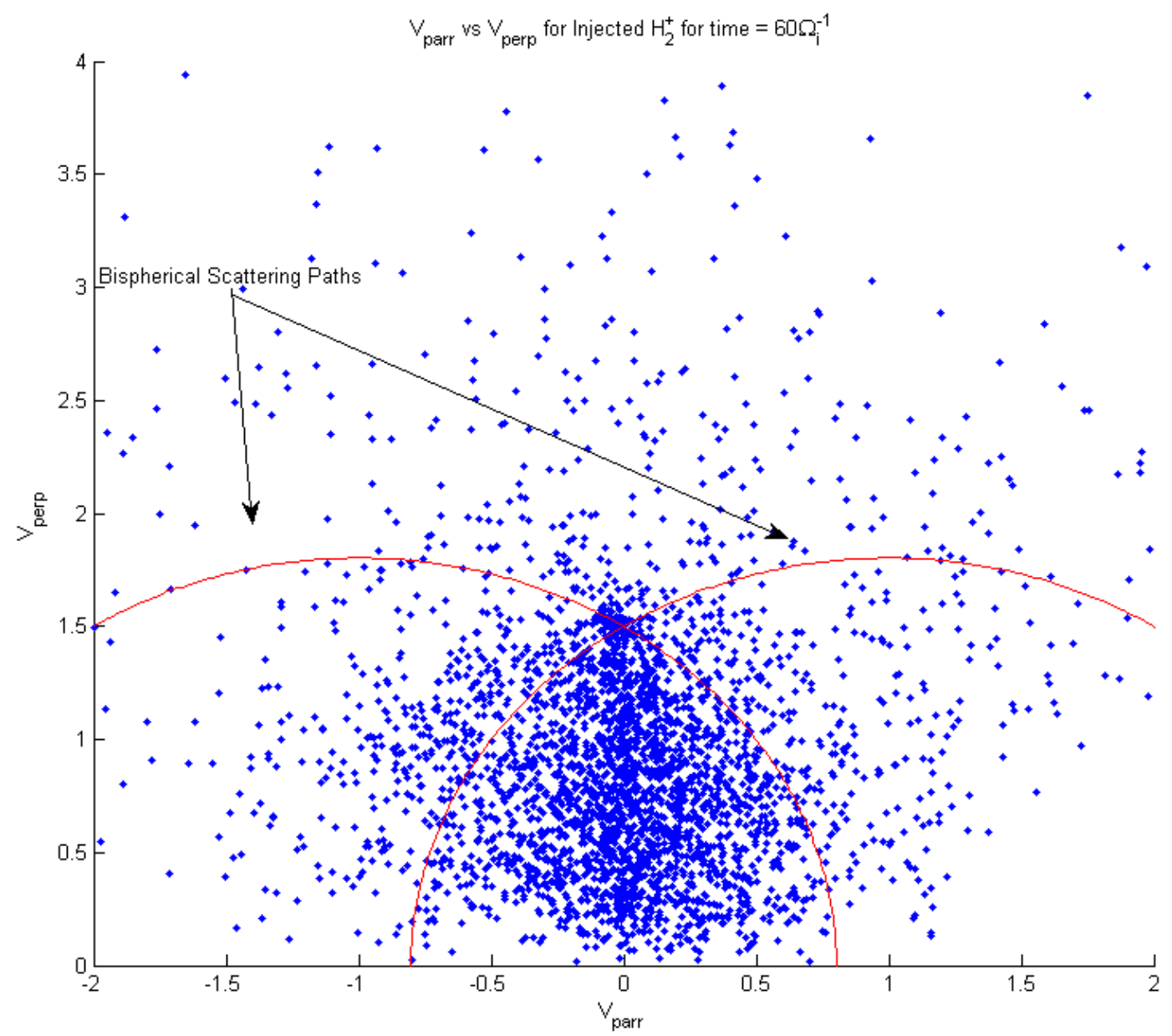

Figure 6.12.: A parallel-perpendicular velocity distribution of the injected hydrogen ions in the high injection rate simulation after $60 \Omega_{i}^{-1}$. The red lines show the scattering paths of a bispherical shell distribution assuming parallel propagating Alfvén waves. 
relatively short simulation duration these effects do not get the opportunity to fully develop and only the beginnings of these interactions are seen. That said, the ions are still scattered to a far larger degree and far more quickly such that some achieve parallel velocities of almost $1.0 V_{A}$ as they are scattered and their overall energy slightly decreases.

Again, the mass loading is the dominant effect on the solar wind plasma, as the injected ions couple to it via the magnetic field they become accelerated. As the injection rate, and hence the coupling effect, is higher more solar wind particles are swept up by the injected plasma compared to other simulations. Additionally the particles which are affected are often accelerated more quickly than in other simulations, this is all due to interactions with the travelling bow wave which is far stronger and more extensive in this simulation.

With a similar comparison to the low injection rate simulation, particles are not accelerated to higher speeds here on average compared to other simulations since that would be energetically improbable under the current setup. Rather, the same energy is transferred to more solar wind particles and is transferred more rapidly. A typical particle will be accelerated to $1.0 V_{A}$ with some reaching as high as $4.0 V_{A}$ and others only $0.4 V_{A}$. Akin to the other particles, all of the acceleration an individual particle experiences occurs over $10-16 \Omega_{i}^{-1}$.

\section{4. $45^{\circ}$ Injection Simulation}

With a $45^{\circ}$ injection angle one of the most common trajectories of the hydrogen ions differs from the other simulations as a typical particle will naturally have a relatively large parallel velocity as a result of its injection geometry. As a result of this most injected ions will have helical trajectories as they stream along field lines and gyrate with their perpendicular velocity. These particles will almost always lose some energy early on in their 'lifetime' to wave generation and as a result of the mass loading process, this energy will usually be drawn initially from the parallel motion of the ions. The energy of an individual particle will often not change significantly after this point although it is not uncommon for the particle to then gain some parallel velocity at the expense of its perpendicular velocity as it is scattered through wave interactions. Occasionally particles can also gain some energy if they have been injected late enough into the simulation that they are positioned to be able to interact in an energetically favourable way with the waves that are produced, this is seen most frequently as a boost to the perpendicular 

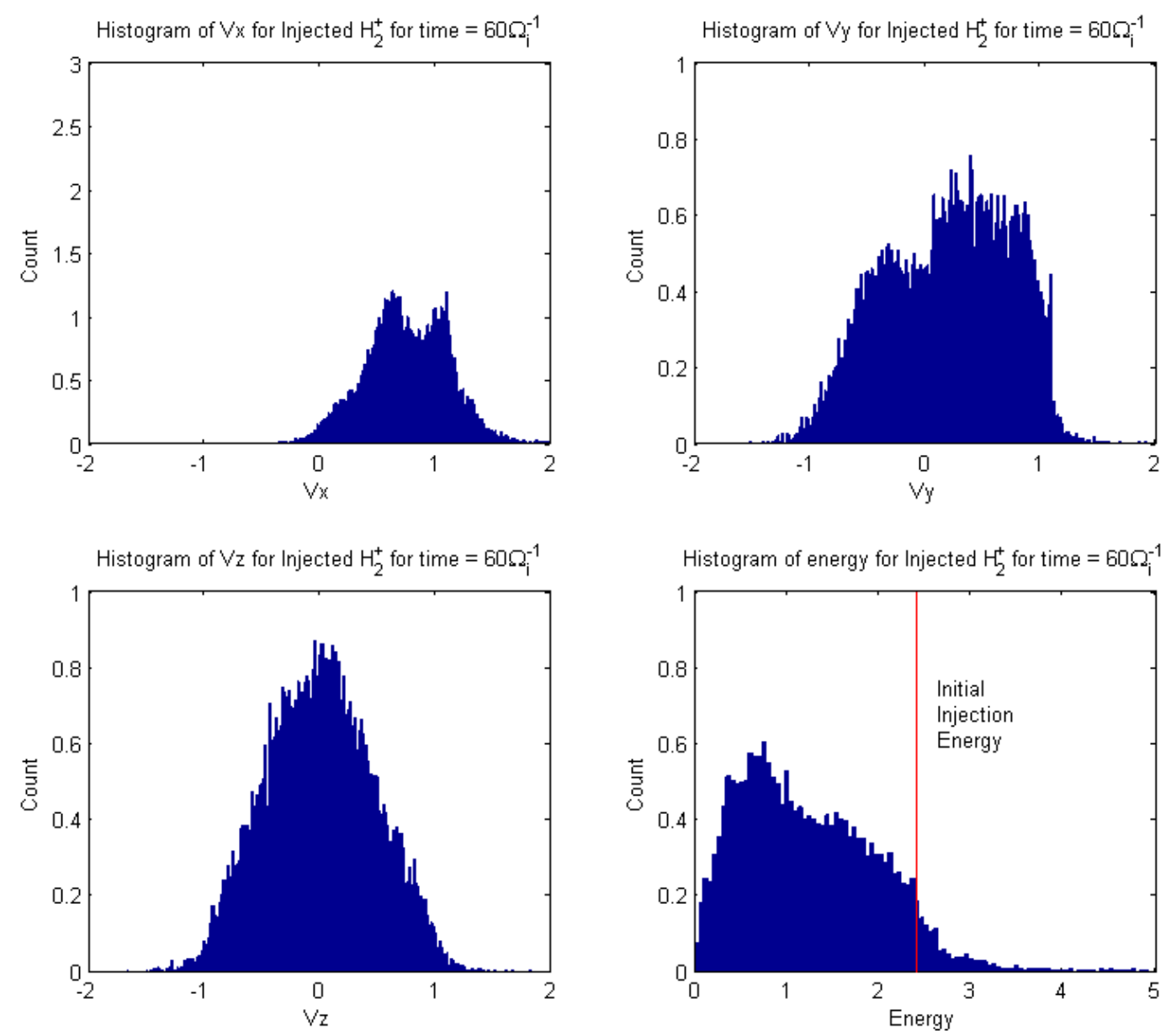

Figure 6.13.: A normalised histogram taken after $60 \Omega_{i}^{-1}$ of the spread of the components of the injected hydrogen's velocity and resulting overall energy in the $45^{\circ}$ injection simulation. The red line on the energy plot shows where a newly injected particle's energy lies.

velocity of a particle. After the injected hydrogen ion has been in the simulation domain for $\approx 20 \Omega_{i}^{-1}$ then it will typically have found its equilibrium and will not then interact strongly with anything thereafter.

Figure 6.13 shows a normalised histogram of the components of the velocity of the injected hydrogen ions. A comparison with Figure 6.1 shows a very similar picture to the reference simulation as the rate of pitch angle scattering is comparable. There is, however, a difference in the rate at which the ions diffuse in energy as there are more ions with a higher energy in this simulation which is due to the parallel component of the injection velocity. This results in a smaller perpendicular velocity and hence a somewhat reduced interaction with the solar wind ions, the parallel component 


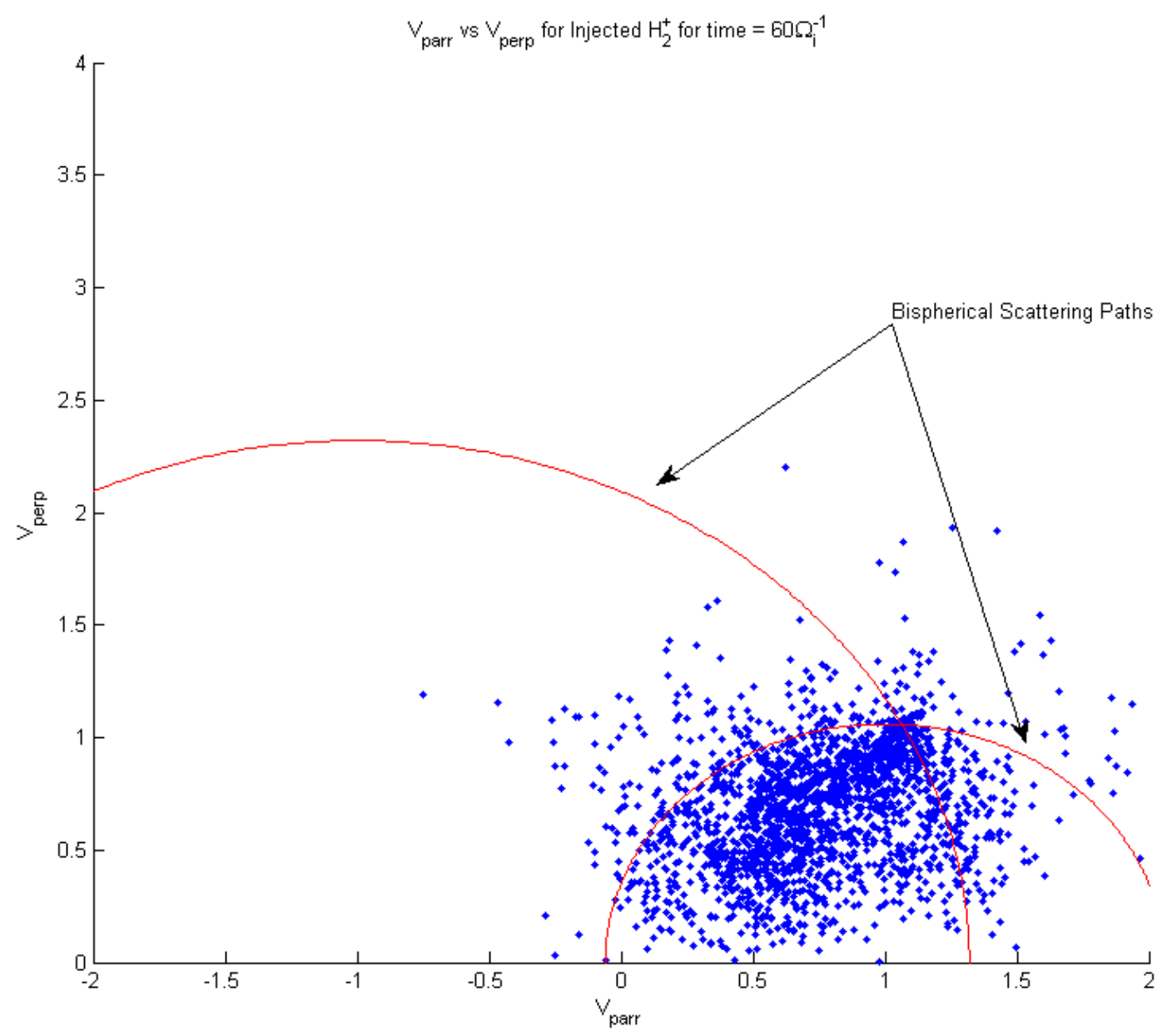

Figure 6.14.: A parallel-perpendicular velocity distribution of the injected hydrogen ions in the $45^{\circ}$ injection simulation after $60 \Omega_{i}^{-1}$. The red lines show the scattering paths of a bispherical shell distribution assuming parallel propagating Alfvén waves.

of velocity drifts slowly towards zero over the duration of the simulation as the scattering process continues. A similar picture of the perpendicular distribution is seen in this case as it flattens and then forms a peaked distribution. The parallel velocity distribution also forms a peak centred on the initial parallel injection velocity which then drifts towards $V_{\|}=0$ but retains a small peak at the injection velocity for the duration of the simulation. The energy distribution behaves in a similar manner to the reference simulation in that it forms a cold Maxwellian distribution.

Figure 6.14 shows that the bispherical distribution continues to describe the evolution of the injected hydrogen's velocity distribution even with a modified angle of injection and appears to do so more accurately than for the reference simulation. 
Nearly the entire population of $\mathrm{H}_{2}^{+}$is contained by at least one of the pair of bispherical shells with very few lying outside. Thus the type of scattering experienced remains the same although we see here that the relative importance of the backward and forward travelling Alfvén waves do depend upon the injection angle as the ions are scattered more strongly by the backward travelling wave in this case.

In this simulation there are also particles which become scattered and have increased parallel velocities. Hydrogen ions which are scattered in this manner will typically lose a small fraction of their perpendicular velocity but can make significant gains to their parallel velocity which is indicative of undergoing a moderate acceleration such as the particle from Figure 6.15. These particles will then continue on helical trajectories but the increased parallel velocity can cause them to pace or even overtake the spacecraft along the $\hat{x}$ co-ordinate (although of course there is a separation along the $\hat{y}$ axis) and such particles are responsible for the extent and persistence, compared to other simulations, of the interaction region along both $\hat{x}$ and the $\hat{y}$ axes. Again, within about $20 \Omega_{i}^{-1}$, the particles have escaped the influence of the interaction region and have been mostly assimilated by the solar wind flow.

Equally, there are particles which, whilst they are initially injected along the $\hat{x}$ axis, are very rapidly reflected and then travel in the $-\hat{x}$ direction for a time. These particles lose the majority of their injection energy during this process and can then either retain their negative parallel velocity or it will slowly 'decay' towards zero. The determining factor appears to be the gyrophase of the particle at the time it undergoes its reflection process. A particle can either be left with an average $V_{y} / V_{z}$ of zero in which case it will retain its parallel velocity or it can have a non-zero average of $V_{y} / V_{z}$ in which case this average will be brought to zero at the expense of the parallel velocity whose average will also 'decay' towards zero. An example of an ion with a non-zero average of $V_{y} / V_{z}$ is shown in Figure 6.16.

It should be noted that the aforementioned hydrogen particles were injected into the simulation early on and are, therefore, located in the 'wings' of the interaction region structure. Particles injected later into the simulation, and therefore into the interaction region more directly, experience a more perturbed environment. These particles are all, seemingly without exception, accelerated a little shortly after injection and this is seen entirely in an oscillation of the parallel velocity component which is likely due to rapid reflections between small wavefronts in the region into which it has been injected after the initial acceleration by the first wave.

The injected nitrogen particles do not significantly interact over the duration of the simulation. They, typically, will merely gyrate about the field lines as they stream along them with a parallel velocity. Due to their larger mass and consequently lower 

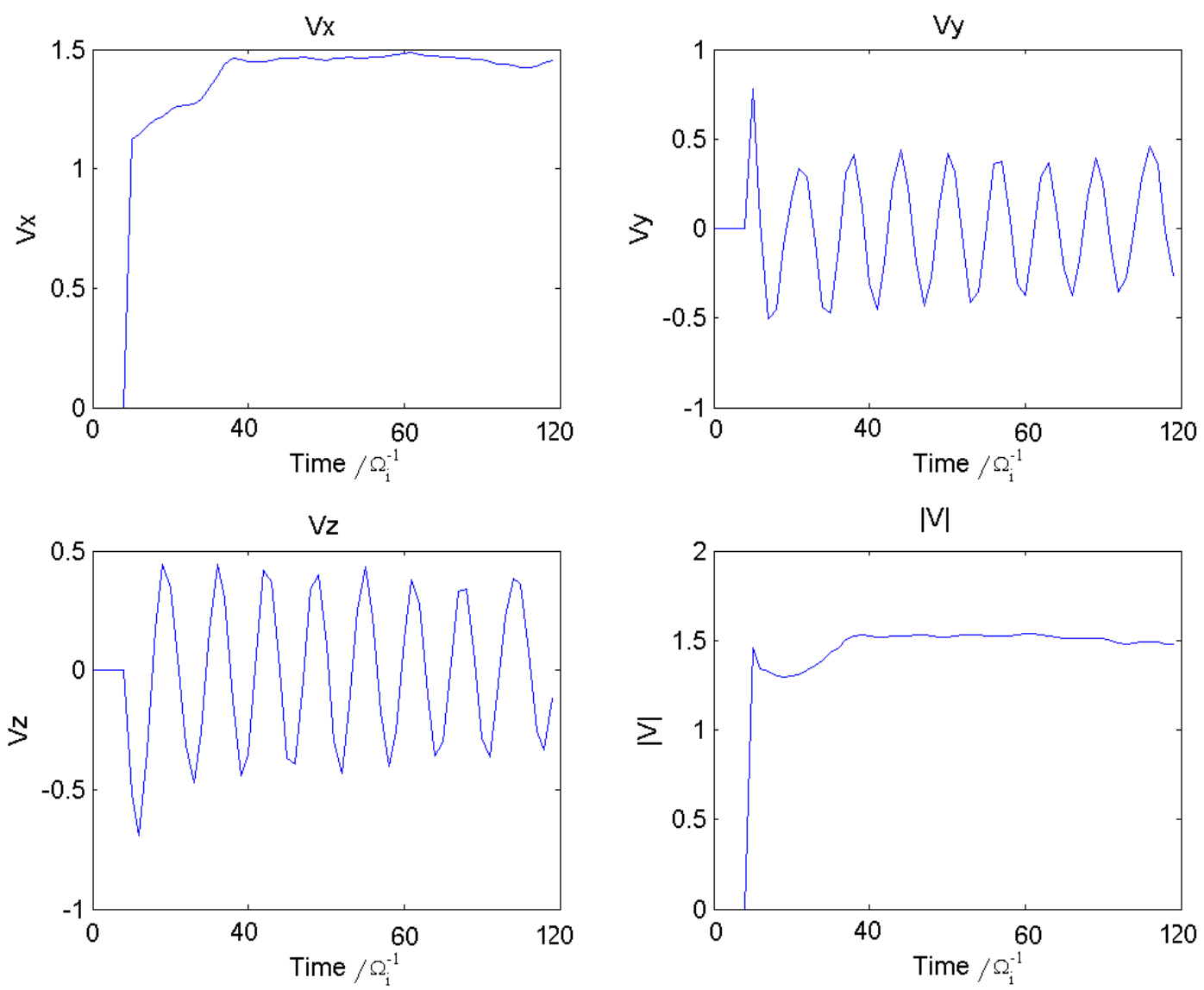

Figure 6.15.: Components and magnitude of an injected hydrogen's velocity. This ion is very strongly scattered into the parallel direction but also makes a net gain of energy, indicative of undergoing an acceleration process. 

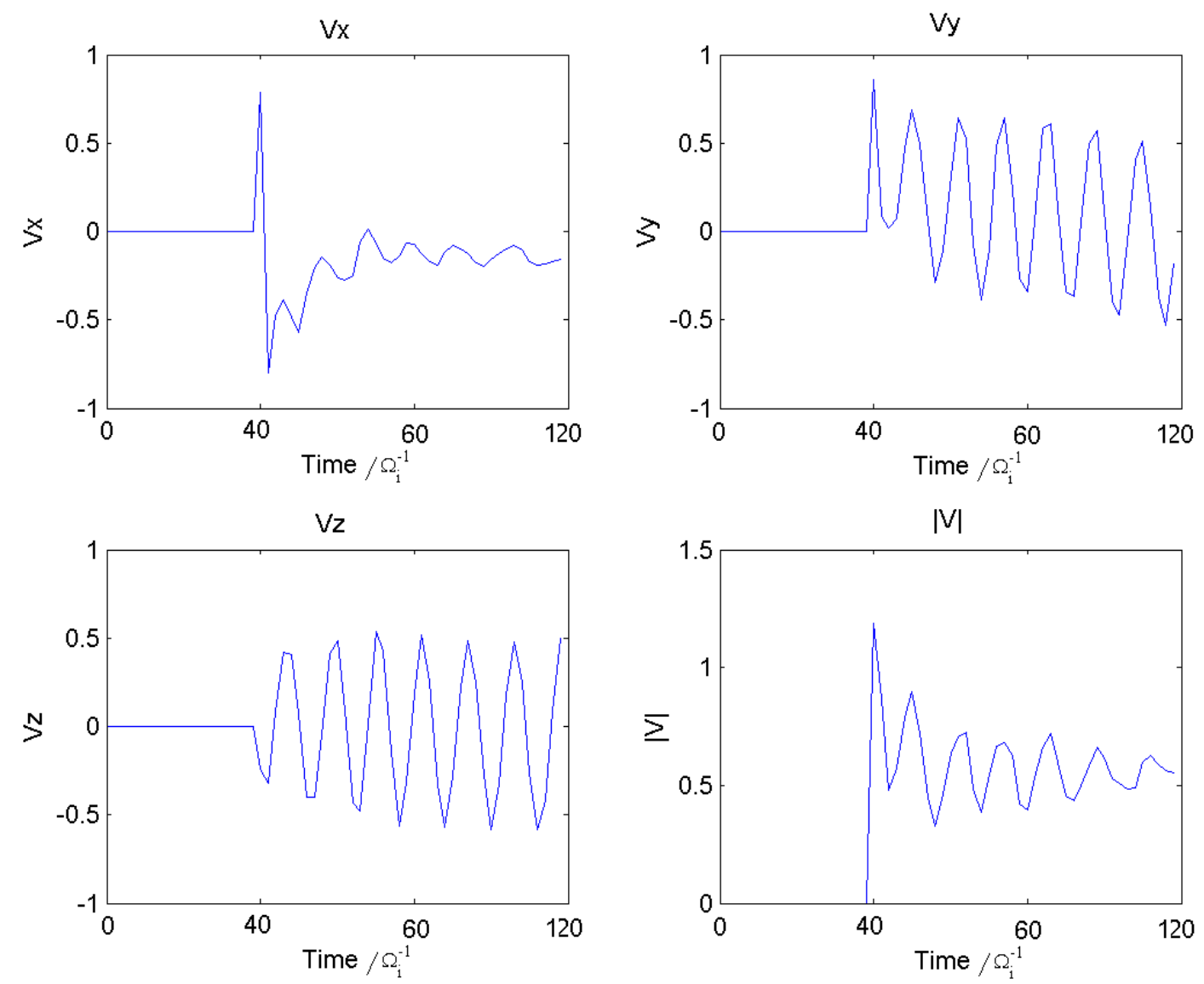

Figure 6.16.: Components and magnitude of an injected hydrogen's velocity. The ion is left with a non-zero average of $V_{y} / V_{z}$ and its parallel velocity decays as this average is brought to zero. 
cyclotron frequency they do not resonate significantly with the higher frequency waves caused and carried by the hydrogen element of the injection. Thus the nitrogen ions only lose a very small fraction of their initial energy over the duration of the simulation and will have trajectories which are almost a textbook helix.

As a result of the injection the solar wind flow is continually mass loaded and thus, in the solar wind frame, will be accelerated. This can be seen as the interaction region passes over the region by the sudden, sharp acceleration of the solar wind protons in the region. After this acceleration the fastest solar wind ions have $|V|=$ $1-3 V_{A}$ with the largest component being in the parallel direction which is shown by Figure 6.17 as the solar wind ion is accelerated by several orders of magnitude within only a few cyclotron times. Not all particles retain their new energies as some are accelerated away from the interaction region and will be decelerated after travelling a short distance and return almost to their initial velocity whilst still others gain a large $V_{\|}$and then are scattered to pitch angles close to $90^{\circ}$ but with a significantly higher perpendicular velocity than they initially had. There are some particles though which are accelerated and then retain the entirety of that energy for the rest of the simulation.

A similar picture can be seen in the $-\hat{x}$ direction as solar wind protons are accelerated anti-parallel to the magnetic field due to the passing of the interaction region. In this case the magnitude of the acceleration is far lower as maximum speeds are of the order $|V|=0.7-1.3 V_{A}$. An additional difference is that the ions all behave in roughly the same manner: they are initially accelerated and gain a modest boost to both their parallel and perpendicular velocity and from that point onwards there is very little change to their energies. They then have a parallel velocity which shows small oscillations as the particle interacts with local waves and a roughly constant perpendicular velocity. This contrasts to the $+\hat{x}$ acceleration where particles reach higher energies and also have a far higher variability to their velocities once accelerated.

\section{5. $0^{\circ}$ Injection Simulation}

Particles are injected along field lines in this simulation so the idealised trajectory of an injected hydrogen ion would simply be a straight line trajectory. Indeed, to a first approximation the ions do follow this type of path although the mutual repulsion of the ions does cause them to have a slight perpendicular velocity. Once the cloud density increases sufficiently these particles can be quickly scattered such that they 

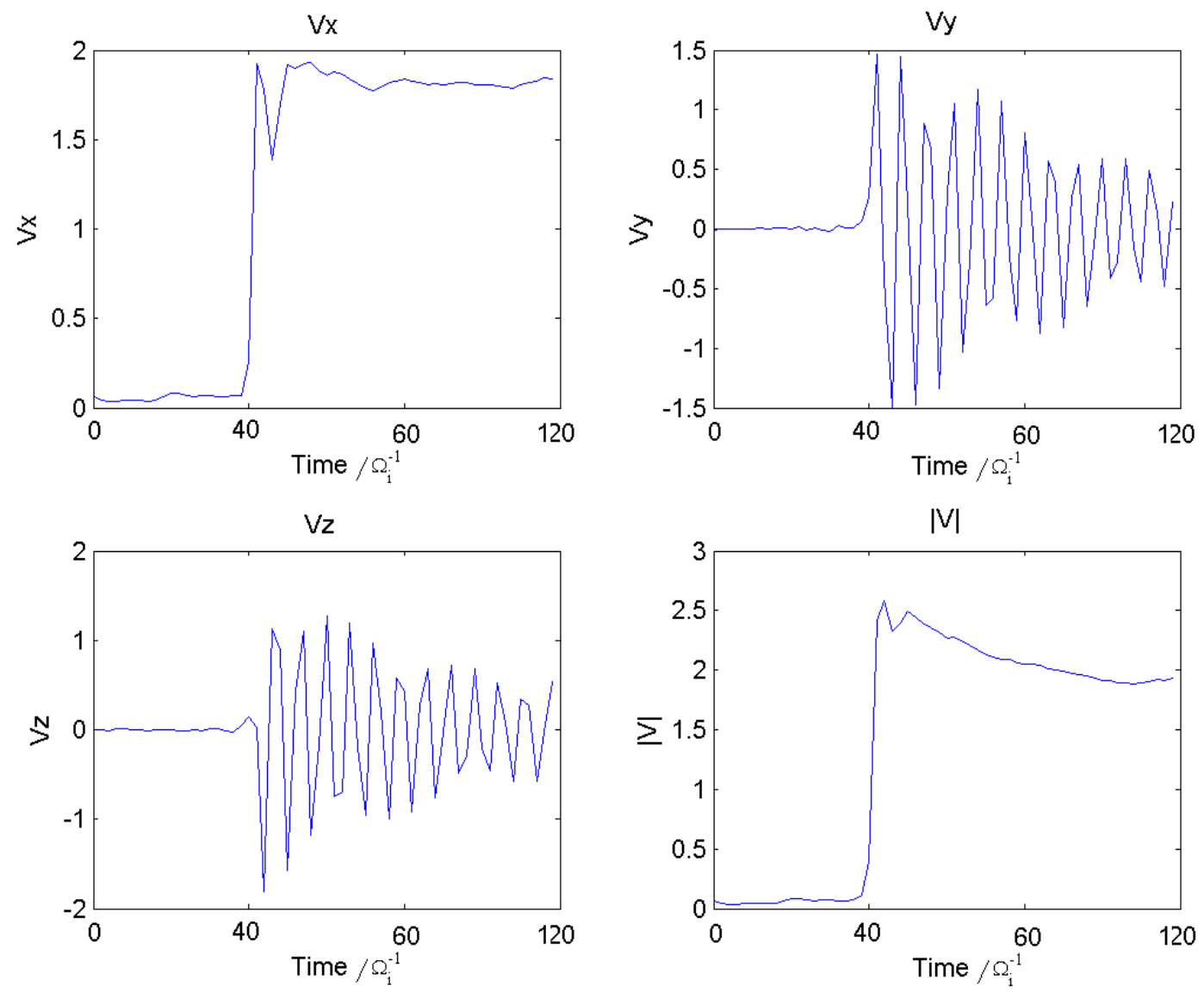

Figure 6.17.: Components and magnitude of a solar wind ion's velocity. The ion encounters the bow wave and is accelerated incredibly rapidly to high speeds. 
have a far larger $V_{\perp}$. In the largest cases the perpendicular velocity can be as high as $1.0 V_{A}$ but is generally a more modest $0.3-0.5 V_{A}$. In most cases this results in an overall slowdown of the particles as they stimulate wave growth but continue onward with helical trajectories.

Figure 6.17 shows a velocity profile qualitatively similar to particles which are injected into the simulation later and are able to interact with both the ion cloud and the produced waves strongly and incredibly rapidly such that they achieve parallel velocities of up to $3 V_{A}$, twice their injection velocity. As the injection is parallel these fast ions are able to stream ahead of the main 'bow wave' of the interaction region, often in clumps. These small clumps of particles then act in a similar manner to the main ion cloud but on a smaller scale as they cause the field lines to curve around them. Equally, whilst some injected hydrogen ions are accelerated, so too are some of them decelerated, these 'detach' from the main ion cloud as their parallel velocity falls significantly below that of most of the injected ion cloud. Again, these travel in small clumps as they fall behind and then spread out and gyrate about the field lines. The trajectories of these particles are similar in that they begin in much the same way, with a trajectory that is almost a straight line. The deceleration of these particles happens early on and they feel the increase in cloud density as a scattering effect as their perpendicular velocity increases by an order of magnitude and they begin to gyrate about the field lines.

Figure 6.18 shows a normalised histogram of the components of the velocity of the injected hydrogen ions. A comparison with Figure 6.1 shows a relatively similar scatter in the perpendicular velocity distribution of this simulation compared to the parallel spread in the reference simulation although the parallel velocity spread of this simulation is significantly less well developed than might have been expected. Due to the parallel injection the ions do not interact at all strongly with the solar wind compared to any of the other simulations and thus the scatter in this case is minimal. This can especially be seen in the energy distribution as a far larger proportion of the injection ions are, in fact, accelerated beyond their initial energy and those that have diffused to lower energies have only done so by a small amount. Thus it can be said that a parallel injection significantly reduces the rate of both pitch angle scattering and energy diffusion of injected ions in this case which is not unexpected. The perpendicular velocity distribution initially is centred on $V_{\perp}=0$ but broadens slightly at the start of the simulation as the ion cloud spreads in space. After approximately $40 \Omega_{i}^{-1}$ the peak suddenly begins to broaden more rapidly as the ion cloud reaches a higher density and the self repulsion is able to give ions a larger perpendicular velocity which enables the pitch angle scattering rate to increase 

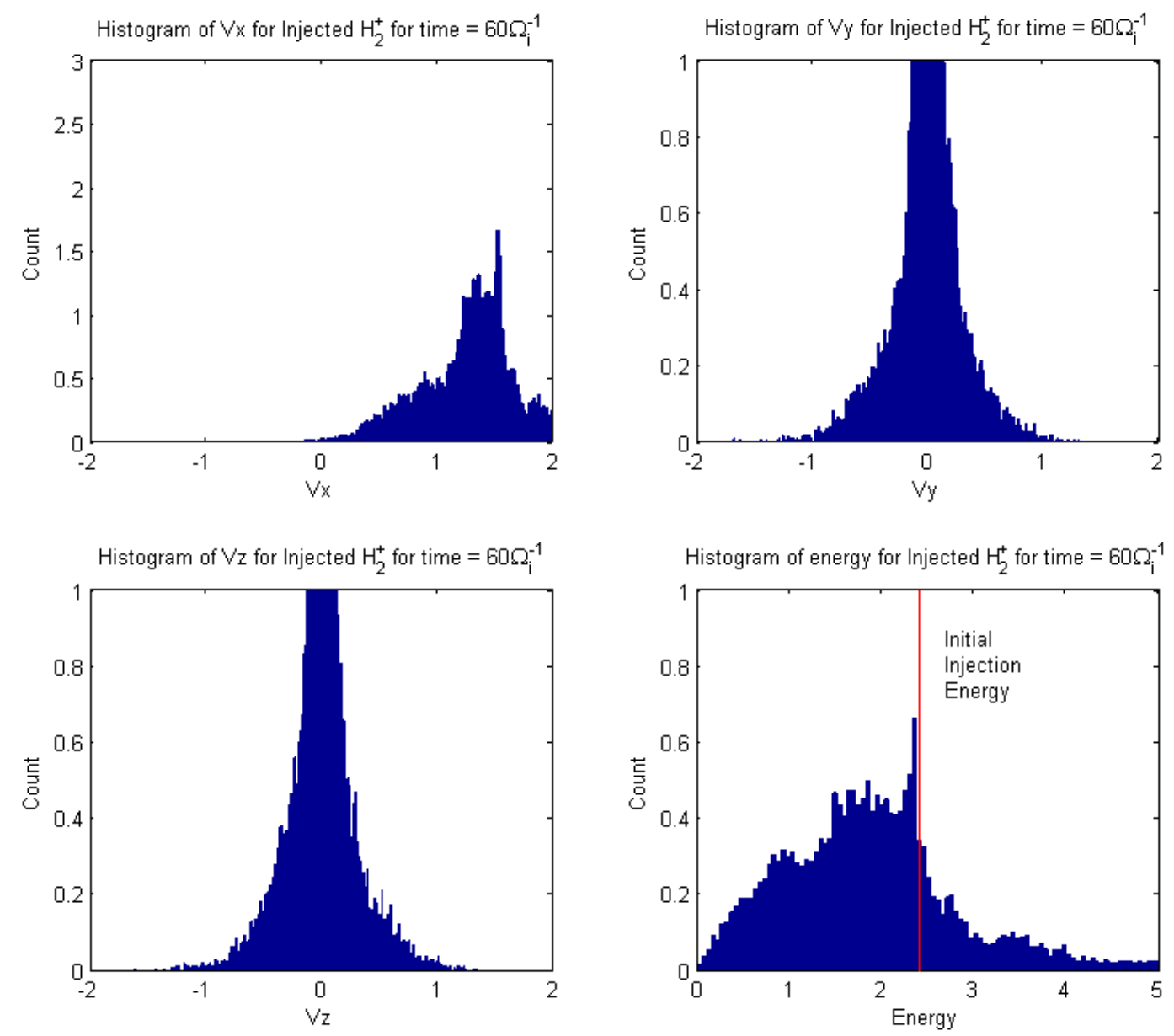

Figure 6.18.: A normalised histogram taken after $60 \Omega_{i}^{-1}$ of the spread of the components of the injected hydrogen's velocity and resulting overall energy in the parallel injection simulation. The red line on the energy plot shows where a newly injected particle's energy lies. 


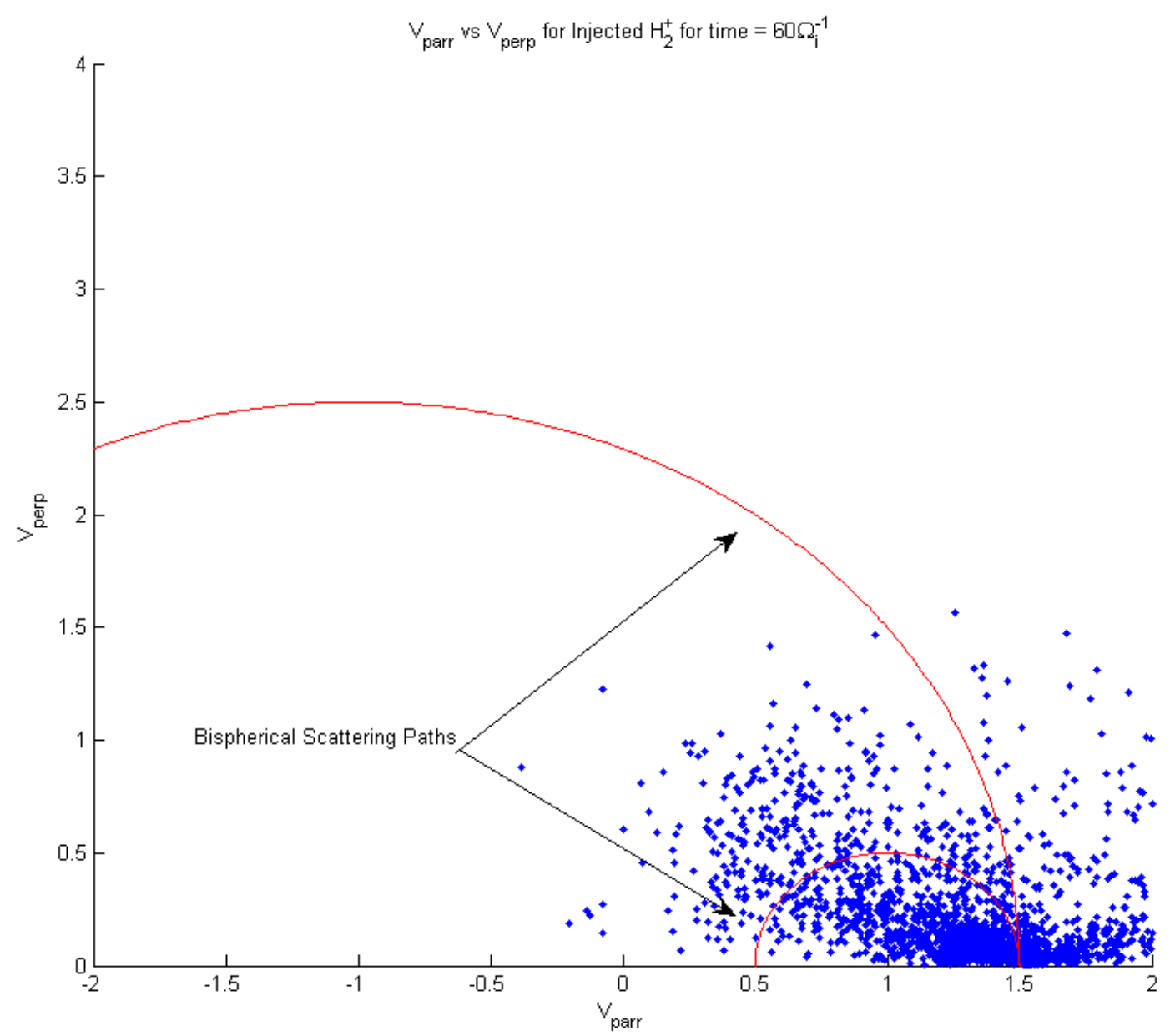

Figure 6.19.: A parallel-perpendicular velocity distribution of the injected hydrogen ions in the parallel injection simulation after $60 \Omega_{i}^{-1}$. The red lines show the scattering paths of a bispherical shell distribution assuming parallel propagating Alfvén waves.

such that the perpendicular distribution is bounded by $\pm V_{A}$. The parallel velocity distribution initially spreads more rapidly to even higher parallel velocities than the ions were injected with and a small peak at $2 V_{A}$ forms which persists throughout the simulation, the distribution then spreads towards $V_{\|}=0$ as scattering progresses but does so less rapidly than for other simulations. This is also evidenced by the evolution of the energy distribution as it becomes a warmer Maxwellian than in other cases with more particles at higher energies overall.

Figure 6.19 shows that, for parallel injections, the bispherical description is not the most appropriate one as the particles have been scattered into a monospherical distribution as is evidenced by most of the ions being contained by a single shell. This 
is due to the relative orientation of the scattering waves compared to the injection direction and also to the interaction between the solar wind ions and the injected ions being weaker than in the reference simulation as the injection is parallel to the ambient magnetic field.

The standard nitrogen trajectory is very similar to the standard hydrogen trajectory in that is travels along the field line almost undisturbed before being slightly influenced by the ion cloud and beginning to gyrate slightly. Other nitrogen ions encounter large and small amplitude magnetic waves which significantly deflect them from their initial trajectory. Particles which encounter the large magnetic field are only slightly deflected and, considering the mass of the ions, moderately slowed. The ions encounter the magnetic field whose orientation is closer to undisturbed and begin gyromotion a short time later. Conversely, those ions which encountered the smaller amplitude wave are scattered less in pitch angle and are therefore deflected to a far greater degree as they reach the undisturbed solar wind sooner and hence begin gyromotion sooner and thus have a much larger deviation from the initial trajectory.

In a similar manner to the hydrogen there are some nitrogen ions which are accelerated in $+\hat{x}$. The increase in their parallel velocity is small as the time for which the nitrogen ions interact with the main cloud is very brief and therefore their parallel velocity has peak values of $V_{\|}=1.7 V_{A}$. The nitrogen ions are also scattered very slightly by their encounter with the wave and begin to gyrate along a radially small helix and again, like the hydrogen, some nitrogen ions lose energy as they are scattered. This deceleration is of a similar magnitude to the acceleration some of the other nitrogen ions experience and they are typically reduced to $V_{\|}=1.3 V_{A}$ which contrasts with the hydrogen which was decelerated less than it was accelerated. These ions are, however, scattered far more on average than the accelerated nitrogen ions.

In a similar manner to the other simulations the solar wind protons can be seen to be accelerated as the ions are deflected in both the $\pm \hat{x}$ directions. These ions then behave in a similar manner to the injected hydrogen ions as they stream along field lines whilst gyrating, describing a helix. The solar wind protons often gain energy consistently for the duration of the simulation as the source of free energy follows behind them. Whilst this energy gain is primarily in the parallel component of the velocity, there is also an element of acceleration involving the perpendicular component. Other ions can also be seen to become trapped between wave fronts in the same way the injected hydrogen was but only in the $-\hat{x}$ direction. For the solar wind protons those ions accelerated in the $+\hat{x}$ direction do not appear to 
become trapped between wave fronts. They only have an increasing parallel velocity although the energy gains are of a similar magnitude. The perpendicular velocity of these ions remains fairly constant throughout the simulation which again contrasts to the fluctuations seen for the protons accelerated in $-\hat{x}$.

\subsection{Conclusions}

We have presented here the findings of an initial investigation into the trajectories of individual particles for several different simulation regimes. We have found that there are many different trajectories that the injection ions may follow. These different trajectories range from the 'standard' pickup ion trajectory of a cycloid in the simulation frame of reference to qualitatively simpler trajectories where the particle is accelerated significantly to particles which encounter the bow wave at oblique angles and have a significantly more complex trajectory as the environment which they experience changes very rapidly. The majority of injected hydrogen ions are found to stimulate wave growth as a result of the mass loading process as is indicated by their overall loss of energy in the solar wind frame although some few ions are accelerated, sometimes significantly, and thus do not contribute significantly to the growth of fluctuations over the duration of the simulation.

Particles are found to undergo pitch angle scattering with differing rates. Compared to the reference simulation the simulation with the lower injection rate showed scattering to a lesser degree and also with a lower rate whereas the simulation with the higher injection rate showed strong scattering early on which persisted throughout the simulation and thus had the largest spread of parallel velocities of all of the simulations with injection performed at $90^{\circ}$ to the magnetic field. The injection parallel to the magnetic field showed less pitch angle scattering as the perpendicular components of the velocity were more strongly peaked than for the $90^{\circ}$ injection and the scattering in energy also differed, more ions were scattered to a higher energy and the diffusion to lower energies was also far less significant The injected ions interacted less strongly with the solar wind than for other injection angles and thus the majority of ions retained the majority of their energy. For the injection at $45^{\circ}$ to the magnetic field the scattering observed was a little more complex as the ions were initially scattered more in the parallel direction around their initial injection velocity component which was directed parallel to the magnetic field. At the same time the scattering in the perpendicular direction was less than observed in the reference simulation. Over time the ions were, however, scattered into a distribution 
tending towards an average parallel velocity centred on zero and with the perpendicular components also centred on zero as predicted by standard pickup theory. The rate of energy diffusion was also slightly lower for this simulation, with more particles having a higher energy.

Particles are found to be scattered into a bispherical distribution as at all times throughout the simulation the majority of injected ions are bounded by the curves which represent the bispherical scattering surfaces of constant energy (constant energy in the frame of the parallel propagating Alfvén waves) although the rate of energy diffusion appears to be comparable to the rate of pitch angle scattering due to the rapid thickening of these shells. The fact that the ions are scattered into a bispherical distribution displays a similarity with cometary ions although the rate at which these shells thicken appears to be faster than is seen at comets. 


\section{Conclusions and Further Work}

This thesis was written with the aim of studying the interactions of a chemical thruster with the solar wind with particular emphasis upon the near solar environment. This is an important area of research given the upcoming Solar Probe Plus (SPP) mission due to launch in 2018 as it will travel to within ten solar radii of the Sun's surface where the space environment will be vastly different to 1 AU. For this reason this work has focused on different interactions a thruster firing may have at ten solar radii.

Thruster firings in general have some extensive literature although to a large extent this is concerned with electric thrusters and the dynamics of ions in the near spacecraft region. Topics such as spacecraft charging, backflow and plume impingement are also covered. Literature concerning chemical thrusters is less extensive and is typically concerned with thruster performance and flow field calculation as well as any spacecraft contamination that may happen. There is a distinct lack of literature which attempts to bridge the gap between chemical thrusters and interactions with the solar wind and especially the effect any thruster has on the solar wind over extended distances.

For this reason this work involved the creation of a model of a generic chemical thruster which provides inputs of ion injection rates to a 3D hybrid plasma simulation code which then tracks the motion of a non-interacting spacecraft as well as the ions produced from the neutral cloud ionisation as they move through the solar wind. These interactions were studied for a range of injection rates and injection angles with respect to the ambient magnetic field

The primary features seen in the simulations as a result of these thruster firings are macroscopic in scale. A bow wave is formed as the injection of newborn ions continues, the intensity and number of wavefronts of the bow wave depends specifically upon the injection rate of the simulation and hence on, effectively, the size of the thruster firing. A larger, longer firing will result in more intense, longer-lived wavefronts which have the potential to become a single 'super wavefront' if the thrust is large enough and multiple wavefronts are given the opportunity to merge. The formation of the bow wave additionally depends upon the ram angle with respect 
to the direction of the magnetic field. The waves are far stronger for injections with a significant component perpendicular to the field lines and weaker for parallel injections although intermediate angles can still have relatively strong interactions.

Due to the mass loading effect, which is the most significant aspect of the thruster firings, the magnetic flux also piles up along the bow wave. This flux pileup is also stronger for larger thrusts in the same way as the bow wave formation, however, there is no component parallel to the undisturbed magnetic field except for those field lines which have been bent by the injection of additional mass. The levels of pileup seen can become quite large, in some cases the fluctuations observed are of the same order of magnitude as the magnetic field's undisturbed strength. Since the flux is frozen to the magnetic field lines and the mass loading effect in the frame of reference of the simulation results in the solar wind ions being locally accelerated whilst those ions further away are unaffected, this means that the field lines will inevitably become curved and will therefore be draped around the cloud of injected material.

The magnitude of this draping is affected by the ability of the field lines to slip over and past the ion cloud and is, therefore, dependant on the size of the thruster firing with larger burns resulting in stronger field line draping patterns. The effects of the draping remain relatively strong until the injection angle becomes close to parallel. At that time the draping pattern requires significantly more time to form as the field lines are no longer dragged along with the ion cloud and are instead pushed aside by the ion cloud's growth. Thus the draping effects in this case are also often weaker.

The combination of the above effects causes the formation of an induced interaction region which is bounded by the combination of magnetic and density perturbations. The size of the interaction region depends rather strongly upon both the size of the thrust and upon its angle with respect to the direction of the magnetic field. For a given angle the interaction region will be larger if the amount of mass loaded onto the solar wind is larger. In addition to this an injection angle of $45^{\circ}$ yields the largest interaction region of all. At this angle the field lines are dragged in the perpendicular direction but are then also moved in the parallel direction due to the draping effects changing the orientation of the local field significantly enough that the motion of the ions may still affect it. This results in a region which has a reasonably large parallel spread due to perpendicular motion but then also has a far larger spread along the perpendicular direction of injection (the $\hat{y}$ axis) due to the parallel component of injection. The interaction region, additionally, whilst bounded in the ram direction by the combination of the bow wave and flux pileup 
regions is typically open in the wake of the spacecraft and ions are free to leave the interaction region in that direction if they possess sufficient velocity.

As a consequence of all of these fairly major perturbations to the solar wind there are magnetosonic waves launched. These are predominantly the large amplitude waves associated with the direct mass loading of the solar wind, i.e., the bow wave. In addition to this though there are also smaller fluctuations which propagate behind the spacecraft. These low level waves are typically in response to the gyration of the injected hydrogen ions. The disturbances caused by the passage of the spacecraft and its ion cloud do not last for significant durations and the solar wind is able to recover rapidly once the core of the cloud has moved on to a different region of space. The solar wind is able to recover more quickly for smaller thrusts and also for parallel injection as it is initially disturbed less.

Curiously there is no evidence of major instabilities present throughout these simulations. From the injection geometry and a comparison with cometary magnetospheres it might have been expected to observe some instabilities which occur there, particularly the electromagnetic ion cyclotron (EMIC) instability which requires an anisotropic velocity distribution. However, despite this, evidence of such instabilities is not seen. This could be due to the scale of the mass loaded onto the solar wind over, initially a relatively small volume, the fact that the spacecraft, and hence the injection region, is moving or perhaps given a longer duration simulation some cyclotron waves associated with the motion of the nitrogen ions may emerge as the simulation durations used are only long enough for the nitrogen to perform a partial gyration.

As a result of the thruster firings there will be significant perturbations present for all scenarios investigated. Due to this, the spacecraft will often experience fluctuations in the ambient conditions which are unrelated to 'natural' processes in the solar wind and are instead a result of the thruster firing only. In the case of a very small burn there will be minimal risk to delicate equipment and sensors, rather, the data acquired for a duration thereafter should be carefully analysed to ensure that the thruster effects have dissipated although such would not be expected to linger for longer than several minutes.

For the larger scale thrusts it may become necessary to cease data taking in order to protect the sensors on the spacecraft. In addition to this the much larger neutral cloud will have a lifetime of tens of minutes and perhaps hours for truly large burns. As a result the ion injection will continue over that time frame and as far as the spacecraft is concerned it will be more likely to pass out of the affected region rather than for the effects of the thruster firing to die down. Thus it becomes likely that for 
larger burns the thrust geometry will have a large influence upon the duration the spacecraft will experience disturbances for and also upon the maximum amplitude of such. It is possible that the worst effects of the thrust can be mitigated somewhat if the thrust is in the spacecraft's wake rather than in the ram direction.

The model used for this series of simulations is by no means perfect. The neutral model is for a generic thruster and therefore will differ from real thrusters in small ways. The ionisation rates used are only predicted rates as there is as yet no accurate data for the processes of interest at such close solar proximity. Further to this not all ionisation pathways are modelled, the pathways with lower rates of ionisation are omitted. These effects combined may result in a different overall ionisation time of the neutral cloud when observed in-situ and hence of a different possible injection rate of newborn ions. The hybrid code models electrons as a charge neutralising fluid and therefore cannot model any electron kinetic effects and hence anything on the very small scale of the electron gyroradius will be lost. Due to computational restraints we cannot study the very long term evolution of the thruster firings. Present simulations have used enhanced ionisation rates in order to accelerate the process that we may observe effects within a reasonable time frame. In order to investigate effects on longer time scales it would be necessary to vastly increase the size of the simulation's domain. Due to this the effects which will be observed by Solar Probe Plus are likely to be a smaller increase to wave fluctuations than predicted here although the strength of the thruster firing is an additional parameter which will have a significant effect upon the perturbations observed.

The work in this thesis inspired a Cluster guest investigator programme in which science data taking continued through some selected thruster firings. The programme was implemented in 2015 and data analysis has not yet commenced. The intention is to validate the simulations against data recorded for thruster firings for future work as data for both electric and magnetic fields at spin resolutions will be available for both long and short duration burns at different times and therefore potentially different solar conditions. This will provide a valuable chance to thoroughly test and tune the model such that its accuracy will be as high as possible for the predictions relevant to Solar Probe Plus.

The spacecraft's thruster firings will introduce a great deal of new material into the solar wind in the area local to Solar Probe Plus. This will directly affect the measurements made by the spacecraft's sensors. The neutral cloud produced will first form a barrier in the visible spectrum but will quickly ionise thereby producing electrostatic noise which may interfere with the Wide Field Imager which attempts to map the Sun's corona in 3D. 
As the interaction progresses and the magnetic and electric fields are increasingly affected the measurements recorded by the FIELDS experiment will no longer be sampling the pristine solar wind but will instead be logging the fluctuations induced by the newborn ions as the neutral cloud becomes increasingly ionised. The magnetometer could be left active during these manoeuvres and predicted data compared with measurements. This in turn, once verified, might enable accurate predictions of the magnitude and duration of the disturbed solar wind. Additionally, it is likely that some of the neutral hydrazine will contact the spacecraft antenna during this time and hence produce additional voltage signals unrelated to space dust.

Similarly, SWEAPS (Solar Wind Electrons Alphas and Protons Investigation) at this time will likely begin to record higher levels of protons, electrons and nitrogen containing compounds as the local region becomes saturated with the hydrazine products. In a similar way to SWEAPS the mass spectrometer on Solar Probe Plus will record increased levels of hydrazine compounds during this time.

Additional further work would involve extending the current area of research to investigate the effects of thruster firings at various other solar distances which would provide the ability to test the model specifically with Solar Probe at times earlier than its first closest approach which will not occur until several years after launch. As with many investigations of this type it will be necessary in the future to perform additional simulations with both a larger simulation domain and longer durations in order to properly simulate the entire lifetime of the thruster firing. 


\section{Appendix A.}

\section{Ionisation Calculations for $\mathbf{H}_{2}$}

As part of the ionisation routines it is useful to define the processes by which $\mathrm{H}_{2}$ can be ionised or otherwise have its structure modified. The following are the ionisation processes considered:

$$
\begin{gathered}
\alpha_{1}: H_{2}+h \nu \rightarrow H+H=2.28 \times 10^{-5} s^{-1} \\
\alpha_{2}: H_{2}+h \nu \rightarrow H^{+}+H+e^{-}=2.77 \times 10^{-5} s^{-1} \\
\alpha_{3}: H_{2}+h \nu \rightarrow H_{2}^{+}+e^{-}=4.87 \times 10^{-5} s^{-1} \\
\beta: H_{2}+e^{-} \rightarrow H_{2}^{+}+2 e^{-}=1.25 \times 10^{-4} s^{-1} \\
\gamma: H_{2}+H^{+} \rightarrow H_{2}^{+}+H^{+}+e^{-}=2.46 \times 10^{-4} s^{-1} \\
\delta: H_{2}+H^{+} \rightarrow H_{2}^{+}+H=2.11 \times 10^{-4} s^{-1} \\
\Lambda: H+h \nu \rightarrow H^{+}+e^{-}=4.5 \times 10^{-7} s^{-1}
\end{gathered}
$$

This results in the following rates of production for the respective species:

- $\lambda_{H_{2}^{+}}=\alpha_{3}+\beta+\gamma+\delta=6.3 \times 10^{-4} s^{-1}$ is the total rate of production of $\mathrm{H}_{2}^{+}$

- $\lambda_{H^{+}}=\alpha_{2}$ is the total rate of production of $\mathrm{H}^{+}$

- $\lambda_{e^{-}}=\alpha_{2}+\alpha_{3}+\beta+\gamma=4.5 \times 10^{-4} s^{-1}$ is the total rate of production of $e^{-}$ 
- $\lambda_{H}=2 \alpha_{1}+\alpha_{2}+\delta=2.8 \times 10^{-4} s^{-1}$ is the total rate of production of $\mathrm{H}$

- $\Lambda_{H_{2}}=\alpha_{1}+\alpha_{2}+\alpha_{3}+\beta+\gamma+\delta=6.8 \times 10^{-4} s^{-1}$ is the total rate of 'decay' or dissociation and ionisation of $\mathrm{H}_{2}$

- $\Lambda_{H}^{\prime}$ is the total rate of ionisation of $\mathrm{H}$

The derivation of the evolution of the species numbers follows.

\section{A.0.1. Rate of decay of $\mathbf{H}_{2}$}

If the total rate of 'decay' of $\mathrm{H}_{2}$ due to all processes is simply $\Lambda_{H_{2}}$ then the number evolution of $\mathrm{H}_{2}$ is:

$$
\begin{aligned}
\frac{d N\left(H_{2}\right)_{t}}{d t} & =-\lambda N \\
N\left(H_{2}\right)_{t} & =N\left(H_{2}\right)_{0} e^{-\Lambda_{H_{2}} t}
\end{aligned}
$$

\section{A.0.2. Rate of production and decay of $\mathrm{H}_{2}^{+}$}

If the total rate of production of $\mathrm{H}_{2}^{+}$is $\lambda_{\mathrm{H}_{2}^{+}}$then the number evolution of $\mathrm{H}_{2}^{+}$is:

$$
\begin{aligned}
\frac{d N\left(H_{2}^{+}\right)_{t}}{d t} & =N\left(H_{2}\right)_{t} \lambda_{H_{2}^{+}}=\lambda_{H_{2}^{+}} N\left(H_{2}\right)_{0} e^{-\Lambda_{H_{2}} t} \\
N\left(H_{2}^{+}\right)_{t} & =\int_{0}^{t} \lambda_{H_{2}^{+}} N\left(H_{2}\right)_{0} e^{-\Lambda_{H_{2}} t} d t \\
& =\lambda_{H_{2}^{+}} N\left(H_{2}\right)_{0} \int_{0}^{t} e^{-\Lambda_{H_{2}} t} d t \\
& =\frac{-\lambda_{H_{2}^{+}} N\left(H_{2}\right)_{0}}{\Lambda_{H_{2}}}\left[e^{-\Lambda_{H_{2}} t}-e^{0}\right] \\
& =\frac{\lambda_{H_{2}^{+}} N\left(H_{2}\right)_{0}}{\Lambda_{H_{2}}}\left(1-e^{-\Lambda_{H_{2}} t}\right)
\end{aligned}
$$

\section{A.0.3. Rate of production and decay of $\mathbf{H}$}

If the total rate of production of $\mathrm{H}$ due to all processes is $\lambda_{H}$ and the rate of ionisation of $\mathrm{H}$ due to all processes is $\Lambda_{H}^{\prime}$ then the number evolution is: 


$$
\begin{aligned}
\frac{d N(H)_{t}}{d t} & =\lambda_{H} N\left(H_{2}\right)_{0} e^{-\Lambda_{H_{2}} t}-N(H)_{t} \Lambda_{H}^{\prime} \\
\frac{d N(H)_{t}}{d t}+N(H)_{t} \Lambda_{H}^{\prime} & =\lambda_{H} N\left(H_{2}\right)_{0} e^{-\Lambda H_{2} t} \\
\frac{d N(H)_{t}}{d t} e^{\Lambda_{H}^{\prime} t}+N(H)_{t} \Lambda_{H}^{\prime} e^{\Lambda_{H}^{\prime} t} & =\lambda_{H} N\left(H_{2}\right)_{0} e^{-\Lambda_{H_{2}} t} \\
\frac{d}{d t}\left(N(H)_{t} e^{\Lambda_{H}^{\prime} t}\right) & =\lambda_{H} N\left(H_{2}\right)_{0} e^{-\Lambda_{H_{2}} t} e^{\Lambda_{H}^{\prime} t} \\
N(H)_{t} e^{\Lambda_{H}^{\prime} t} & =\int_{0}^{t} \lambda_{H} N\left(H_{2}\right)_{0} e^{\left(\Lambda_{H}^{\prime}-\Lambda_{H_{2}}\right) t} \\
N(H)_{t} e^{\Lambda_{H}^{\prime} t} & =\frac{\lambda_{H} N\left(H_{2}\right)_{0}}{\Lambda_{H}^{\prime}-\Lambda_{H_{2}}}\left[e^{\left(\Lambda_{H}^{\prime}-\Lambda_{H_{2}}\right) t}-1\right] \\
N(H)_{t} & =\frac{\lambda_{H} N\left(H_{2}\right)_{0}}{\Lambda_{H}^{\prime}-\Lambda_{H_{2}}}\left[e^{-\Lambda_{H_{2}} t}-e^{-\Lambda_{H}^{\prime} t}\right]
\end{aligned}
$$

\section{A.0.4. Rate of production of $\mathbf{H}^{+}$}

If the total rate of production of $\mathrm{H}^{+}$due to ionisation of $\mathrm{H}_{2}$ is $\lambda_{H^{+}}=\alpha_{2}$ and the total rate of production due to the ionisation of $\mathrm{H}$ is $\Lambda_{H}^{\prime}$ then the number evolution is:

$$
\begin{aligned}
\frac{d N\left(H^{+}\right)_{t}}{d t} & =N\left(H_{2}\right)_{t} \alpha_{2}+N(H)_{t} \Lambda_{H}^{\prime} \\
\frac{d N\left(H^{+}\right)_{t}}{d t} & =N\left(H_{2}\right)_{0} \alpha_{2} e^{-\Lambda_{H_{2}} t}+\frac{\Lambda_{H}^{\prime} \lambda_{H} N\left(H_{2}\right)_{0}}{\Lambda_{H}^{\prime}-\Lambda_{H_{2}}}\left(e^{-\Lambda_{H_{2}} t}-e^{-\Lambda_{H}^{\prime} t}\right) \\
N\left(H^{+}\right)_{t} & =\frac{N\left(H_{2}\right)_{0} \alpha_{2}}{\Lambda_{H_{2}}}\left(1-e^{-\Lambda_{H_{2}} t}\right) \\
& +\frac{\Lambda_{H}^{\prime} \lambda_{H} N\left(H_{2}\right)_{0}}{\Lambda_{H}^{\prime}-\Lambda H_{2}}\left[\left(\frac{-e^{-\Lambda_{H_{2}} t}}{\Lambda_{H_{2}}}+\frac{e^{-\Lambda_{H}^{\prime} t}}{\Lambda_{H}^{\prime}}\right)-\left(\frac{-1}{\Lambda_{H_{2}}}+\frac{1}{\Lambda_{H}^{\prime}}\right)\right] \\
N\left(H^{+}\right)_{t} & \left.=N\left(H_{2}\right)_{0}\left[\frac{\alpha_{2}}{\Lambda_{H_{2}}}\left(1-e^{-\Lambda_{H_{2}} t}\right)+\frac{\Lambda_{H}^{\prime} \lambda_{H}}{\Lambda_{H}^{\prime}-\Lambda_{H_{2}}}\left(\frac{1-e^{-\Lambda_{H_{2}} t}}{\Lambda_{H_{2}}}\right)-\left(\frac{1-e^{-\Lambda_{H}^{\prime}}}{\Lambda_{H}^{\prime}}\right)\right)\right]
\end{aligned}
$$

Where the first term in the square brackets on the right is due to the ionisation of $\mathrm{H}_{2}$ and the second term is due to the secondary ionisation of $\mathrm{H}$ atoms.

\section{A.0.5. Rate of production of electrons}

If the total rate of production of electrons from all processes is $\lambda_{e}$ then the number evolution is: 


$$
\begin{aligned}
\frac{d N(e)_{t}}{d t} & =N\left(H_{2}\right)_{t} \lambda_{e}+N(H)_{t} \Lambda_{H}^{\prime} \\
\frac{d N(e)_{t}}{d t} & =N\left(H_{2}\right)_{0}\left[\lambda_{e} e^{-\Lambda_{H_{2}} t}+\frac{\Lambda_{H}^{\prime} \lambda_{H}}{\Lambda_{H}^{\prime}-\Lambda_{H_{2}}}\left(e^{-\Lambda_{H_{2}} t}-e^{-\Lambda_{H}^{\prime} t}\right)\right] \\
N(e)_{t} & =N\left(H_{2}\right)_{0}\left[\left(\frac{-\lambda_{e} e^{-\Lambda_{H_{2}} t}}{\Lambda_{H_{2}}}\right)_{0}^{t}+\frac{\Lambda_{H}^{\prime} \lambda_{H}}{\Lambda_{H}^{\prime}-\Lambda_{H_{2}}}\left(\frac{-e^{-\Lambda_{H_{2}} t}}{\Lambda_{H_{2}}}+\frac{e^{-\Lambda_{H}^{\prime} t}}{\Lambda_{H}^{\prime}}\right)_{0}^{t}\right] \\
N(e)_{t} & =N\left(H_{2}\right)_{0}\left[\frac{\lambda_{e}}{\Lambda_{H_{2}}}\left(1-e^{-\Lambda_{H_{2}} t}\right)+\frac{\Lambda_{H}^{\prime} \lambda_{H}}{\Lambda_{H}^{\prime}-\Lambda_{H_{2}}}\left(\frac{-e^{-\Lambda_{H_{2}} t}}{\Lambda_{H_{2}}}+\frac{e^{-\Lambda_{H}^{\prime} t}}{\Lambda_{H}^{\prime}}+\frac{1}{\Lambda_{H_{2}}}-\frac{1}{\Lambda_{H}^{\prime}}\right)\right] \\
N(e)_{t} & \left.=N\left(H_{2}\right)_{0}\left[\frac{\lambda_{e}}{\Lambda_{H_{2}}}\left(1-e^{-\Lambda_{H_{2}} t}\right)+\frac{\Lambda_{H}^{\prime} \lambda_{H}}{\Lambda_{H}^{\prime}-\Lambda_{H_{2}}}\left(\frac{1-e^{-\Lambda_{H_{2}}}}{\Lambda_{H_{2}}}\right)-\left(\frac{1-e^{-\Lambda_{H} t}}{\Lambda_{H}^{\prime}}\right)\right)\right]
\end{aligned}
$$

Where the first term in the square brackets on the right is due to processes involving $\mathrm{H}_{2}$ and the second term is due to the ionisation of $\mathrm{H}$ atoms. 


\section{Appendix B.}

\section{Ionisation Calculations for $\mathrm{N}_{2}$}

The ionisation processes considered for $\mathrm{N}_{2}$ are as follows:

$$
\begin{gathered}
\mu: N_{2}+h \nu \rightarrow N_{2}^{+}+e^{-}=7.00 \times 10^{-7} s^{-1} \\
\eta: N_{2}+e^{-} \rightarrow N_{2}^{+}+2 e^{-}=3.52 \times 10^{-7} s^{-1} \\
\theta: N_{2}+h \nu \rightarrow 2 N=1.30 \times 10^{-7} s^{-1} \\
\sigma: N+h \nu \rightarrow N^{+}+e^{-}=8.30 \times 10^{-7} s^{-1}
\end{gathered}
$$

\section{B.0.6. Rate of Decay of $\mathrm{N}_{2}$}

If the total rate of 'decay' of $\mathrm{N}_{2}$ due to all processes is simply $\Lambda$ then the number evolution of $\mathrm{N}_{2}$ is:

$$
\begin{aligned}
\frac{d N\left(N_{2}\right)_{t}}{d t} & =-\lambda N \\
N\left(N_{2}\right)_{t} & =N\left(N_{2}\right)_{0} e^{-\Lambda t}
\end{aligned}
$$

\section{B.0.7. Rate of production and decay of $\mathrm{N}_{2}^{+}$}

If the total rate of production of $\mathrm{N}_{2}^{+}$is $\lambda_{N_{2}^{+}}$then the number evolution of $\mathrm{N}_{2}^{+}$is: 


$$
\begin{aligned}
\frac{d N\left(N_{2}^{+}\right)_{t}}{d t} & =N\left(N_{2}\right)_{t} \lambda_{N_{2}^{+}}=\lambda_{N_{2}^{+}} N\left(N_{2}\right)_{0} e^{-\Lambda t} \\
N\left(N_{2}^{+}\right)_{t} & =\int_{0}^{t} \lambda_{N_{2}^{+}} N\left(N_{2}\right)_{0} e^{-\Lambda t} d t \\
& =\lambda_{N_{2}^{+}} N\left(N_{2}\right)_{0} \int_{0}^{t} e^{-\Lambda t} d t \\
& =\lambda_{N_{2}^{+}} N\left(N_{2}\right)_{0}\left[\frac{-e^{-\Lambda t}}{\Lambda}\right]_{0}^{t} \\
& =\lambda_{N_{2}^{+}} N\left(N_{2}\right)_{0}\left[\frac{1-e^{-\Lambda t}}{\Lambda}\right] \\
N\left(N_{2}^{+}\right)_{t} & =\frac{\lambda_{N_{2}^{+}} N\left(N_{2}\right)_{0}}{\Lambda}\left[1-e^{-\Lambda t}\right]
\end{aligned}
$$

\section{B.0.8. Rate of production and ionisation of $\mathrm{N}$}

If the rate of production of $\mathrm{N}$ due to all processes is $\lambda_{N}$ and the rate of ionisation of $\mathrm{N}$ due to all processes is $\lambda_{N^{+}}$then the number evolution of $\mathrm{N}$ is:

$$
\begin{aligned}
\frac{d N(N)_{t}}{d t} & =N\left(N_{2}\right)_{0} e^{-\Lambda t} \lambda_{N}-N(N)_{t} \lambda_{N^{+}} \\
\frac{d N(N)_{t}}{d t}+N(N)_{t} \lambda_{N^{+}} & =N\left(N_{2}\right)_{0} e^{-\Lambda t} \lambda_{N} \\
\frac{d}{d t}\left(e^{\lambda_{N^{+}} t} N(N)_{t}\right) & =e^{\lambda_{N^{+}} t} N\left(N_{2}\right) e^{-\Lambda t} \lambda_{N} \\
\frac{d}{d t}\left(e^{\lambda_{N^{+}} t} N(N)_{t}\right) & =\lambda_{N} N\left(N_{2}\right)_{0} e^{\lambda_{N^{+}} e^{-\Lambda t}} \\
& \left.\left.=\lambda_{N} N\right) N_{2}\right)_{0} e^{\left(\lambda_{N^{+}}-\Lambda\right) t} \\
e^{\lambda_{N^{+}} t} N(N)_{t} & =\int_{0}^{t} \lambda_{N} N\left(N_{2}\right)_{0} e^{\left(\lambda_{N^{+}}-\Lambda\right) t} d t \\
e^{\lambda_{N^{+}} t} N(N)_{t} & =\lambda_{N} N\left(N_{2}\right)_{0} \int_{0}^{t} e^{\left(\lambda_{N^{+}}-\Lambda\right) t} d t \\
e^{\lambda_{N}+t} N(N)_{t} & =\lambda_{N} N\left(N_{2}\right)_{0}\left[\frac{e^{\left(\lambda_{N^{+}}-\Lambda\right) t}}{\lambda_{N^{+}}-\Lambda}\right]_{0}^{t} \\
& =\lambda_{N} N\left(N_{2}\right)_{0}\left[\frac{e^{\left(\lambda_{N^{+}}-\Lambda\right) t}-1}{\lambda_{N^{+}}-\Lambda}\right] \\
e^{\lambda_{N^{+}} t} N(N)_{t} & =\frac{\lambda_{N} N\left(N_{2}\right)_{0}}{\lambda_{N+}-\Lambda}\left[e^{\lambda_{N^{+}} t} e^{-\Lambda t}-1\right] \\
N(N)_{t} & =\frac{\lambda_{N} N\left(N_{2}\right)_{0}}{\lambda_{N+}}\left[e^{-\Lambda t}-e^{-\lambda_{N}+t}\right]
\end{aligned}
$$




\section{B.0.9. Rate of production of $\mathrm{N}^{+}$}

If the total rate of production of $\mathrm{N}^{+}$due to ionisation of $\mathrm{N}$ is $\lambda_{N^{+}}$then the number evolution is:

$$
\begin{aligned}
\frac{d N\left(N^{+}\right)}{d t} & =N(N)_{t} \lambda_{N^{+}} \\
\frac{d N\left(N^{+}\right)}{d t} & =\overbrace{\frac{\lambda_{N^{+}} \lambda_{N} N\left(N_{2}\right)_{0}}{\lambda_{N^{+}}-\Lambda}}^{\mathrm{A}}\left[e^{-\Lambda t}-e^{-\lambda_{N^{+}}}\right] \\
N\left(N^{+}\right)_{t} & =A \int_{0}^{t}\left[e^{-\Lambda t}-e^{-\lambda_{N^{+}}}\right] d t \\
N\left(N^{+}\right)_{t} & =A\left[\frac{-e^{-\Lambda t}}{\Lambda}+\frac{e^{-\lambda_{N^{+}}}}{\lambda_{N^{+}}}\right]_{0}^{t} \\
& =A\left[\left(\frac{e^{-\lambda_{N^{+}} t}}{\lambda_{N^{+}}}-\frac{e^{-\Lambda t}}{\Lambda}\right)-\left(\frac{1}{\lambda_{N^{+}}}-\frac{1}{\Lambda}\right)\right] \\
& =A\left[\left(\frac{e^{-\lambda_{N^{+}}}-1}{\lambda_{N^{+}}}\right)+\left(\frac{1-e^{-\Lambda t}}{\Lambda}\right)\right] \\
N\left(N^{+}\right)_{t} & =\frac{\lambda_{N^{+}} \lambda_{N} N\left(N_{2}\right)_{0}}{\lambda_{N^{+}}-\Lambda}\left[\frac{e^{-\lambda_{N^{+}}}-1}{\lambda_{N^{+}}}+\frac{1-e^{-\Lambda t}}{\Lambda}\right]
\end{aligned}
$$




\section{References}

H. Alfvén. On the origin of the solar system. 1954.

M. G. Baring. Diffusive shock acceleration: The fermi mechanism. In GiraudHeraud, Y. and Tran Thanh Vanastro, J., editors, Very High Energy Phenomena in the Universe, volume 97. 1997.

W. Baumjohann, A. Matsuoka, K.H. Glassmeier, C.T. Russell, T. Nagai, M. Hoshino, T. Nakagawa, A. Balogh, J.A. Slavin, R. Nakamura, and W. Magnes. The magnetosphere of mercury and its solar wind environment: Open issues and scientific questions. Advances in Space Research, 38(4):604 - 609, 2006. ISSN 0273-1177. doi: http://dx.doi.org/10.1016/j.asr.2005.05.117. URL http: //www.sciencedirect.com/science/article/pii/S0273117705007787. Mercury, Mars and Saturn.

P.A. Bernhardt, R. A. Roussel-Dupre, M. B. Pongratz, G. Haerendel, A. Valenzuela, D. A. Gurnett, and R. R. Anderson. Observations and theory of the ampte magnetotail barium releases. JGR, 92(A6), 1987.

P.C. Birch and S.C. Chapman. Detailed structure and dynamics in particle-incell simulations of the lunar wake. PHYSICS OF PLASMAS, 8(10):4551-4559, October 2001. URL http://wrap.warwick.ac.uk/11741/.

N.F. Blagoveshchenskaya, T.D. Borisova, and V.A. Kornienko. Medium-scale ionospheric wave processes during the $\{\mathrm{CRRES}\}$ barium releases. Advances in Space Research, 21(5):749 - 752, 1998. ISSN 0273-1177. doi: http://dx.doi.org/10. 1016/S0273-1177(97)01017-X. URL http://www. sciencedirect.com/science/ article/pii/S027311779701017X. Active Experiments in Space Plasmas.

X. Blanco-Cano, C.T. Russell, D.E. Huddleston, and R.J. Strangeway. Ion cyclotron waves near Io. Planetary and Space Science, 49(1011):1125 - 1136, 2001. ISSN 0032-0633. doi: http://dx.doi.org/10.1016/S0032-0633(01)00020-4. URL http: //www.sciencedirect.com/science/article/pii/S0032063301000204. Magnetosphere of the Outer Planets Part II.

X. Blanco-Cano, N. Omidi, and C.T. Russel. Simulations of solar wind interaction with magnetized asteroids: Comparison with galileo observations near gaspra and ida. Journal of Geophysical Research, 108(A5):1216-1229, 2003.

S. A. Boardsen and J. A. Slavin. Search for pick-up ion generated $\mathrm{Na}^{+}$cyclotron waves at Mercury. Geophysical Research Letters, 34(22), 2007. ISSN 1944-8007. doi: 10.1029/2007GL031504. URL http://dx .doi .org/10 . 1029/2007GL031504. 
Scott A. Boardsen, James A. Slavin, Brian J. Anderson, Haje Korth, David Schriver, and Sean C. Solomon. Survey of coherent $1 \mathrm{~Hz}$ waves in Mercury's inner magnetosphere from messenger observations. Journal of Geophysical Research: Space Physics, 117(A12), 2012. ISSN 2156-2202. doi: 10.1029/2012JA017822. URL http://dx.doi.org/10.1029/2012JA017822. A00M05.

O. Bolin, N. Brenning, C. M. Swenson, and F. Primdahl. Crit II electric and magnetic observations inside and outside an ionizing neutral jet. Journal of Geophysical Research: Space Physics, 101(A9):19729-19744, 1996. ISSN 2156-2202. doi: 10.1029/96JA01284. URL http://dx.doi.org/10.1029/96JA01284.

A. L. Brinca. Generation and Nonlinear Evolution of Cometary Waves, pages 45-76. TERRAPUB, 1997.

Armando L. Brinca and Bruce T. Tsurutani. Survey of low-frequency electromagnetic waves stimulated by two coexisting newborn ion species. Journal of Geophysical Research: Space Physics, 93(A1):48-58, 1988. ISSN 2156-2202. doi: 10. 1029/JA093iA01p00048. URL http://dx.doi.org/10.1029/JA093iA01p00048.

D A Bryant. Ion release experiments in the solar wind. Plasma Physics and Controlled Fusion, 27(12A):1369, 1985. URL http://stacks.iop.org/0741-3335/ $27 / i=12 \mathrm{~A} / \mathrm{a}=004$.

D. Burgess. Virtual instruments for space plasmas. ESA SP, 371:179-185, 1995.

K.M. Bury and T.W. Kerslake. The effect of reaction control system thruster plume impingement on orion service module solar array power production. In Proceedings of the Sixth International Energy Conversion Engineering Conference. American Institute of Aeronautics and Astronautics, July 2008.

C. Cai and I. Boyd. Theoretical and numerical study of free-molecular flow problems. Journal of Fluid Mechanics, 44(3):619-624, 2007.

C. Cai and I. D. Boyd. Collisionless gas expanding into vacuum. Journal of Spacecraft and Rockets, 44:1326-1330, November 2007.

C. Cai and L. Wang. Gaskinetic analytical and numerical studies on rarefied unsteady planar jet flows. Physica Scripta, 88(4), 2013.

S. V. Chalov. Helium pickup ion focusing cone as an indicator of the interstellar flow direction. Monthly Notices of the Royal Astronomical Society: Letters, 443(1):L25-L28, 2014. doi: 10.1093/mnrasl/slu074. URL http://mnrasl. oxfordjournals.org/content/443/1/L25. abstract.

O. Chanrion. Electrostatic charging simulation of spacecraft using a stationary plasma thruster in geostationary plasmic environment. In R.A. Harris, editor, Proceedings of the Seventh International Spacecraft Charging Technology Conference, page 509. ESA, April 2001. 
P. Cheouxdamas, J.M. Stephan, S. Castejon, A. Romashkp, V.F. Petrusevich, and D. Volkov. Validation of an ion thruster plume impingement software by in-flight measurements. In Michael Perry, editor, Proceedings of the Second European Spacecraft Propulsion Conference, page 499. ESA, May 1997.

Adam Clemens and David Burgess. Pickup ion processes associated with spacecraft thrusters: Implications for solar probe plus. Physics of Plasmas, 23(3):032901, 2016. doi: http://dx.doi.org/10.1063/1.4942938. URL http://scitation .aip. org/content/aip/journal/pop/23/3/10.1063/1.4942938.

A. J. Coates, A. D. Johnstone, and F. M. Neubauer. Cometary ion pressure anisotropies at comets Halley and Grigg-Skjellerup. Journal of Geophysical Research: Space Physics, 101(A12):27573-27583, 1996. ISSN 2156-2202. doi: 10.1029/96JA02524. URL http://dx.doi.org/10.1029/96JA02524.

A.J. Coates. Ion pickup at comets. Advances in Space Research, 33(11): 1977 - 1988, 2004. ISSN 0273-1177. doi: http://dx.doi.org/10.1016/j.asr. 2003.06.029. URL http://www.sciencedirect.com/science/article/pii/ S0273117704000213. Comparative Magnetospheres.

M. M. Cowee and S. P. Gary. Electromagnetic ion cyclotron wave generation by planetary pickup ions: One-dimensional hybrid simulations at sub-alfvnic pickup velocities. Journal of Geophysical Research: Space Physics, 117(A6), 2012. ISSN 2156-2202. doi: 10.1029/2012JA017568. URL http://dx.doi.org/10.1029/ 2012JA017568. A06215.

M. M. Cowee, C. T. Russell, and R. J. Strangeway. One-dimensional hybrid simulations of planetary ion pickup: Effects of variable plasma and pickup conditions. Journal of Geophysical Research: Space Physics, 113(A8), 2008. ISSN 2156-2202. doi: 10.1029/2008JA013066. URL http://dx.doi.org/10.1029/2008JA013066. A08220.

M. M. Cowee, S. P. Gary, and H. Y. Wei. Pickup ions and ion cyclotron wave amplitudes upstream of Mars: First results from the 1d hybrid simulation. Geophysical Research Letters, 39(8), 2012. ISSN 1944-8007. doi: 10.1029/2012GL051313. URL http://dx.doi.org/10.1029/2012GL051313. L08104.

N. F. Cramer, F. Verheest, and S. V. Vladimirov. Instabilities of alfvn and magnetosonic waves in dusty cometary plasmas with an ion ring beam. Physics of Plasmas, 6(1):36-43, 1999. doi: http://dx.doi.org/10.1063/1.873255. URL http: //scitation.aip.org/content/aip/journal/pop/6/1/10.1063/1.873255.

T. E. Cravens, A. Hoppe, S. A. Ledvina, and S. McKenna-Lawlor. Pickup ions near mars associated with escaping oxygen atoms. Journal of Geophysical Research: Space Physics, 107(A8):SMP 7-1-SMP 7-10, 2002. ISSN 2156-2202. doi: 10. 1029/2001JA000125. URL http://dx.doi.org/10.1029/2001JA000125. 
T.E. Cravens and T.I. Gombosi. Cometary magnetospheres: a tutorial. Advances in Space Research, 33(11):1968 - 1976, 2004. ISSN 0273-1177. doi: http://dx.doi. org/10.1016/j.asr.2003.07.053. URL http://www. sciencedirect.com/science/ article/pii/S0273117704000201. Comparative Magnetospheres.

Shannon M. Curry, Janet Luhmann, Yingjuan Ma, Michael Liemohn, Chuanfei Dong, and Takuya Hara. Comparative pick-up ion distributions at mars and venus: Consequences for atmospheric deposition and escape. Planetary and Space Science, 115:35 - 47, 2015. ISSN 0032-0633. doi: http://dx.doi.org/10.1016/j. pss.2015.03.026. URL http://www. sciencedirect.com/science/article/pii/ S0032063315001026. Solar wind interaction with the terrestrial planets.

P.A. Delamere, D.W. Swift, and H.C. Stenbaek-Nielsen. A three-dimensional hybrid code simulation of the december 1984 solar wind ampte release. Geophysical Research Letters, 26(18):2837-2840, 1999.

P.A. Delamere, D.W. Swift, and H.C. Stenbaek-Nielsen. An explanation of the ion cloud morphology in the crres plasma injection experiments. JGR, 106(A10): 21289-21295, 2001.

Vincent Dols, Peter A. Delamere, Fran Bagenal, William S. Kurth, and William R. Paterson. Asymmetry of Io's outer atmosphere: Constraints from five galileo flybys. Journal of Geophysical Research: Planets, 117(E10), 2012. ISSN 2156-2202. doi: 10.1029/2012JE004076. URL http://dx.doi.org/10.1029/2012JE004076. E10010.

S. D. Drell, H. M. Foley, and M. A. Ruderman. Drag and propulsion of large satellites in the ionosphere: An Alfvén propulsion engine in space. Journal of Geophysical Research, 70(13):3131-3145, 1965. ISSN 2156-2202. doi: 10.1029/ JZ070i013p03131. URL http://dx.doi .org/10.1029/JZ070i013p03131.

R.E. Ergun, D.M. Malaspina, S.D. Bale, J.P. McFadden, D.E. Larson, F.S. Mozer, N. Meyer-Vernet, M. Maksimovic, P.J. Kellogg, and J.R. Wygant. Spacecraft charging and ion wake formation in the near-sun environment. Physics of Plasmas, 17(072903), 2010.

Xiaohua Fang, Michael W. Liemohn, Andrew F. Nagy, Yingjuan Ma, Darren L. De Zeeuw, Janet U. Kozyra, and Thomas H. Zurbuchen. Pickup oxygen ion velocity space and spatial distribution around mars. Journal of Geophysical Research: Space Physics, 113(A2), 2008. ISSN 2156-2202. doi: 10.1029/2007JA012736. URL http://dx.doi.org/10.1029/2007JA012736. A02210.

G. Gloeckler, E. Möbius, J. Geiss, M. Bzowski, S. Chalov, H. Fahr, D. R. McMullin, H. Noda, M. Oka, D. Ruciński, R. Skoug, T. Terasawa, R. von Steiger, A. Yamazaki, and T. Zurbuchen. Observations of the helium focusing cone with pickup ions. AA, 426(3):845-854, 2004. doi: 10.1051/0004-6361:20035768. URL http://dx.doi.org/10.1051/0004-6361:20035768. 
J. D. Gaffey, D. Winske, and C. S. Wu. Time scales for formation and spreading of velocity shells of pickup ions in the solar wind. Journal of Geophysical Research: Space Physics, 93(A6):5470-5486, 1988. ISSN 2156-2202. doi: 10.1029/ JA093iA06p05470. URL http://dx.doi.org/10.1029/JA093iA06p05470.

A. A. Galeev, K. I. Gringauz, S. I. Klimov, A. P. Remizov, R. Z. Sagdeev, S. P. Savin, A. Yu. Sokolov, M. I. Verigin, K. Szegö, M. Tátrallyay, R. Grard, Ye. G. Yeroshenko, M. Mogilevsky, W. Riedler, and K. Schwingenschuh. Physical processes in the vicinity of the cometopause interpreted on the basis of plasma, magnetic field, and plasma wave data measured on board the vega 2 spacecraft. Journal of Geophysical Research: Space Physics, 93(A7):7527-7531, 1988. ISSN 2156-2202. doi: 10.1029/JA093iA07p07527. URL http://dx.doi .org/10.1029/ JA093iA07p07527.

A.A. Galeev and R.Z. Sagdeev. Alfvén waves in a space plasma and its role in the solar wind interaction with comets. Astrophysics and Space Science, 144 (1-2):427-438, 1988. ISSN 0004-640X. doi: 10.1007/BF00793196. URL http: //dx.doi.org/10.1007/BF00793196.

S. P. Gary. Electromagnetic ion/ion instabilities and their consequences in space plasmas - A review. , 56:373-415, May 1991. doi: 10.1007/BF00196632.

S.P. Gary and C.D. Madland. Electromagnetic ion instabilities in a cometary environment. JGR, 93(A1), 1988.

S.P. Gary and R. Sinha. Electromagnetic waves and instabilities from cometary ion velocity shell distributions. JGR, 94(A7), 1989.

Daniel J. Gershman, George Gloeckler, Jason A. Gilbert, Jim M. Raines, Lennard A. Fisk, Sean C. Solomon, Edward C. Stone, and Thomas H. Zurbuchen. Observations of interstellar helium pickup ions in the inner heliosphere. Journal of Geophysical Research: Space Physics, 118(4):1389-1402, 2013. ISSN 2169-9402. doi: 10.1002/jgra.50227. URL http://dx.doi.org/10.1002/jgra. 50227.

J. Giacalone and J. R. Jokipii. Spatial variation of accelerated pickup ions at co-rotating interaction regions. Geophysical Research Letters, 24(14):1723-1726, 1997. ISSN 1944-8007. doi: 10.1029/97GL01631. URL http://dx.doi.org/10. 1029/97GL01631.

P. W. Gingell, T. Sundberg, and D. Burgess. The impact of a hot sodium ion population on the growth of the kelvin-helmholtz instability in mercury's magnetotail. Journal of Geophysical Research: Space Physics, 120(7):5432-5442, 2015. ISSN 2169-9402. doi: 10.1002/2015JA021433. URL http://dx.doi.org/10.1002/ 2015JA021433. 2015JA021433.

K. H. Glassmeier, U. Motschmann, C. Mazelle, F. M. Neubauer, K. Sauer, S. A. Fuselier, and M. H. Acua. Mirror modes and fast magnetoacoustic waves 
near the magnetic pileup boundary of comet p/halley. Journal of Geophysical Research: Space Physics, 98(A12):20955-20964, 1993. ISSN 2156-2202. doi: 10.1029/93JA02582. URL http://dx.doi.org/10.1029/93JA02582.

K. H. Glassmeier, B. T. Tsurutani, and F. M. Neubauer. Adventures in Parameter Space: A Comparison of Low-Frequency Plasma Waves at Comets, pages 77-119. TERRAPUB, 1997.

G. Gloeckler, J. Geiss, E. C. Roelof, L. A. Fisk, F. M. Ipavich, K. W. Ogilvie, L. J. Lanzerotti, R. von Steiger, and B. Wilken. Acceleration of interstellar pickup ions in the disturbed solar wind observed on Ulysses. Journal of Geophysical Research: Space Physics, 99(A9):17637-17643, 1994. ISSN 2156-2202. doi: 10. 1029/94JA01509. URL http://dx.doi.org/10.1029/94JA01509.

George Gloeckler and Johannes Geiss. Interstellar and inner source pickup ions observed with SWICS on Ulysses. Space Science Reviews, 86(1-4):127-159, 1998. ISSN 0038-6308. doi: 10.1023/A:1005019628054. URL http://dx.doi.org/10 . 1023/A\%3A1005019628054.

George Gloeckler and Johannes Geiss. Heliospheric and interstellar phenomena deduced from pickup ion observations. Space Science Reviews, 97(1-4):169-181, 2001. ISSN 0038-6308. doi: 10.1023/A:1011867320416. URL http://dx.doi. org/10.1023/A\%3A1011867320416.

R. Grard, F. Scarf, J. G. Trotignon, and M. Mogilevsky. A comparison between wave observations performed in the environments of Comets Halley and GiacobiniZinner. In E. J. Rolfe and B. Battrick, editors, Diversity and Similarity of Comets, volume 278 of ESA Special Publication, pages 97-105, September 1987.

J.M. Grebowsky, D.H. Crider, D.S. Intriligator, R.E. Hartle, and M.H. Acua. Venus/Mars pickup ions and ionosheath wave structures. Advances in Space Research, 33(2):176 - 181, 2004. ISSN 0273-1177. doi: http://dx.doi.org/10.1016/j. asr.2003.04.014. URL http://www. sciencedirect.com/science/article/pii/ S0273117703010548. Planetary Atmospheres, Ionospheres and Plasma Interactions.

D. A. Gurnett, R. R. Anderson, P. A. Bernhardt, H. Lühr, G. Haerendel, O. H. Bauer, H. C. Koons, and R. H. Holzworth. Plasma waves associated with the first AMPTE magnetotail barium release. Geophysical Research Letters, 13(7): 644-647, 1986. ISSN 1944-8007. doi: 10.1029/GL013i007p00644. URL http: //dx.doi.org/10.1029/GL013i007p00644.

D.A. et al Gurnett. Plasma waves associated with the AMPTE artificial comet. Geophysical Research Letters, 12(12):851-854, 1985.

G. Haerendel, G. Paschmann, W. Baumjohann, and C.W. Carlson. Dynamics of the AMPTE artificial comet. Nature, 320(24):720-723, 1986. 
J.B. Harold and A.B. Hassam. A simulation of the december 1984 solar wind AMPTE release. Geophysical Research Letters, 18(2):135-138, 1991.

B. Häusler, L. J. Woolliscroft, R. R. Anderson, D. A. Gurnett, R. H. Holzworth, H. C. Koons, O. H. Bauer, G. Haerendel, R. A. Treumann, P. J. Christiansen, A. G. Darbyshire, M. P. Gough, S. R. Jones, A. J. Norris, H. Lühr, and N. Klöcker. Plasma waves observed by the IRM and UKS spacecraft during the AMPTE solar wind lithium releases: Overview. Journal of Geophysical Research: Space Physics, 91(A2):1283-1299, 1986. ISSN 2156-2202. doi: 10.1029/JA091iA02p01283. URL http://dx.doi.org/10.1029/JA091iA02p01283.

D. E. Huddleston, R. J. Strangeway, X. Blanco-Cano, C. T. Russell, M. G. Kivelson, and K. K. Khurana. IO - Jupiter Interaction: Waves Generated by Pickup Ions. Advances in Space Research, 26:1513-1518, 2000. doi: 10.1016/S0273-1177(00) 00091-0.

D. E. Hunton and J. S. Machuzak. Thruster firing effects in the shuttle environment: 2. positive ion composition. Journal of Geophysical Research: Space Physics, 99 (A3):4011-4022, 1994. ISSN 2156-2202. doi: 10.1029/93JA03060. URL http: //dx.doi.org/10.1029/93JA03060.

Philip A. Isenberg and Martin A. Lee. A dispersive analysis of bispherical pickup ion distributions. Journal of Geophysical Research: Space Physics, 101(A5):1105511066, 1996. ISSN 2156-2202. doi: 10.1029/96JA00293. URL http://dx.doi. org/10.1029/96 JA00293.

T. Itoh and K. Hirao. The Sakigake and Suisei Encounters with comet Halley (GRL 13(8) 1986), page 45. 1998.

A.D. Johnstone. Cometary ion pickup processes: Halley and Grigg-Skjellerup compared. Advances in Space Research, 16(4):11 - 18, 1995. ISSN 02731177. doi: http://dx.doi.org/10.1016/0273-1177(95)00202-P. URL http: //www . sciencedirect.com/science/article/pii/027311779500202P. Comparative Studies of Magnetospheric Phenomena.

J.R. Jokipii and J. Giacalone. The acceleration of pickup ions. Space Science Reviews, 78(1-2):137-148, 1996. ISSN 0038-6308. doi: 10.1007/BF00170800. URL http://dx.doi.org/10.1007/BF00170800.

D Jones and M J Rycroft. The plasma physics of the active magnetospheric particle tracer explorers (AMPTE) mission. Plasma Physics and Controlled Fusion, 26 (12A):1395, 1984. URL http://stacks.iop.org/0741-3335/26/i=12A/a=005.

M. Jugroot and J.K. Harvey. Particle simulation of the main chamber of a kaufmantype ion thruster. In R.A. Harris, editor, Proceedings of the 3rd International Conference on Spacecraft Propulsion. ESA, October 2000. 
R. Kallenbach, J. Geiss, G. Gloeckler, and R. von Steiger. Pick-up ion measurements in the heliosphere - a review. Astrophysics and Space Science, 274(1-2):97-114, 2000. URL http://hdl.handle.net/2027.42/41954.

F. Kazeminezhad, J. M. Dawson, and R. Bingham. Simulations and qualitative analysis of the AMPTE experiments. Journal of Geophysical Research: Space Physics, 98(A6):9493-9502, 1993. ISSN 2156-2202. doi: 10.1029/92JA02006. URL http://dx.doi.org/10.1029/92JA02006.

P. L. Koehn, T. H. Zurbuchen, G. Gloeckler, R. A. Lundgren, and L. A. Fisk. Measuring the plasma environment at Mercury: The fast imaging plasma spectrometer. Meteoritics Planetary Science, 37(9):1173-1189, 2002. ISSN 1945-5100. doi: 10.1111/j.1945-5100.2002.tb00887.x. URL http://dx.doi.org/10.1111/j . 1945-5100.2002.tb00887.x.

Harri Laakso. Electric fields and cold electrons in the vicinity of comet Halley. Journal of Geophysical Research: Space Physics, 96(A5):7731-7757, 1991. ISSN 21562202. doi: 10.1029/90JA02459. URL http://dx.doi.org/10.1029/90JA02459.

G. Le, C. T. Russell, S. P. Gary, E. J. Smith, W. Riedler, and K. Schwingenschuh. ULF waves at comets Halley and Giacobini-Zinner: Comparison with simulations. Journal of Geophysical Research: Space Physics, 94(A9):11989-11995, 1989. ISSN 2156-2202. doi: 10.1029/JA094iA09p11989. URL http://dx.doi.org/10.1029/ JA094iA09p11989.

Stephen A. Ledvina, Stephen H. Brecht, and Janet G. Luhmann. Ion distributions of 14 amu pickup ions associated with Titan's plasma interaction. Geophysical Research Letters, 31(17), 2004. ISSN 1944-8007. doi: 10.1029/2004GL019861. URL http://dx.doi.org/10.1029/2004GL019861. L17S10.

Martin A. Lee, Vitali D. Shapiro, and Roald Z. Sagdeev. Pickup ion energization by shock surfing. Journal of Geophysical Research: Space Physics, 101(A3):47774789, 1996. ISSN 2156-2202. doi: 10.1029/95JA03570. URL http://dx.doi. org/10.1029/95JA03570.

J. S. Leisner, C. T. Russell, M. K. Dougherty, X. Blanco-Cano, R. J. Strangeway, and C. Bertucci. Ion cyclotron waves in Saturn's E ring: Initial Cassini observations. Geophysical Research Letters, 33(11), 2006. ISSN 1944-8007. doi: 10.1029/2005GL024875. URL http://dx . doi .org/10 . 1029/2005GL024875. L11101.

Timur J. Linde and Tamas I. Gombosi. Interstellar dust filtration at the heliospheric interface. Journal of Geophysical Research: Space Physics, 105(A5):10411-10417, 2000. ISSN 2156-2202. doi: 10.1029/1999JA900149. URL http://dx.doi.org/ 10.1029/1999JA900149.

K. Liou, R. B. Torbert, and G. Haerendel. Momentum coupling in the CRIT II critical ionization velocity experiment. Journal of Geophysical Research: Space 
Physics, 101(A9):19649-19657, 1996. ISSN 2156-2202. doi: 10.1029/96JA00393. URL http://dx.doi.org/10.1029/96JA00393.

Q. Lu, L. Shan, T. Zhang, G. P. Zank, Z. Yang, M. Wu, A. Du, and S. Wang. The Role of Pickup Ions on the Structure of the Venusian Bow Shock and its Implications for the Termination Shock. , 773:L24, August 2013. doi: 10.1088/ 2041-8205/773/2/L24.

J. G. Luhmann, S. A. Ledvina, J. G. Lyon, and C. T. Russell. Venus O + pickup ions: Collected PVO results and expectations for Venus Express. , 54:1457-1471, November 2006. doi: 10.1016/j.pss.2005.10.009.

J.G. Luhmann. The solar wind interaction with venus. Space Science Reviews, 44 (3-4):241-306, 1986. ISSN 0038-6308. doi: 10.1007/BF00200818. URL http: //dx.doi.org/10.1007/BF00200818.

Hermann Lühr, Wolfgang Baumjohann, and Thomas A. Potemra. The AMPTE lithium releases in the solar wind: A possible trigger for geomagnetic pulsations. Geophysical Research Letters, 17(13):2301-2304, 1990. ISSN 1944-8007. doi: 10. 1029/GL017i013p02301. URL http://dx.doi.org/10.1029/GL017i013p02301.

M. A. Malkov and L. O'C. Drury. Nonlinear theory of diffusive acceleration of particles by shock waves. Reports on Progress in Physics, 64:429-481, 2001.

U. Mall, E. Kirsch, K. Cierpka, B. Wilken, A. Söding, F. Neubauer, G. Gloeckler, and A. Galvin. Direct observation of lunar pick-up ions near the moon. Geophysical Research Letters, 25(20):3799-3802, 1998. ISSN 1944-8007. doi: 10.1029/1998GL900003. URL http://dx.doi.org/10.1029/1998GL900003.

R. Marchand, Y. Miyake, H. Usui, J. Deca, G. Lapenta, J. C. Matéo-Vélez, R. E. Ergun, A. Sturner, V. Gnot, A. Hilgers, and S. Markidis. Cross-comparison of spacecraft-environment interaction model predictions applied to solar probe plus near perihelion. Physics of Plasmas, 21(6):062901, 2014. doi: http://dx.doi.org/ 10.1063/1.4882439. URL http://scitation.aip.org/content/aip/journal/ pop/21/6/10.1063/1.4882439.

P. Matthews, A. Current advance method and cyclic leapfrog for $2 \mathrm{~d}$ multispecies hybrid plasma simulations. Journal of Computational Physics, 112(1):102-116, 1994.

Ronald H. Miller, S. Peter Gary, Dan Winske, and Tamas I. Gombosi. Pitch angle scattering of cometary ions into monospherical and bispherical distributions. Geophysical Research Letters, 18(6):1063-1066, 1991a. ISSN 1944-8007. doi: 10.1029/91GL01047. URL http://dx.doi.org/10.1029/91GL01047.

Ronald H. Miller, Tamas I. Gombosi, S. Peter Gary, and Dan Winske. The directional dependence of magnetic fluctuations generated by cometary ion pickup. Journal of Geophysical Research: Space Physics, 96(A6):9479-9490, 1991b. ISSN 
2156-2202. doi: 10.1029/91JA00158. URL http://dx.doi.org/10.1029/ 91JA00158.

E. Möbius, D. Hovestadt, B. Klecker, M. Scholer, G. Gloeckler, F. M. Ipavich, and H. Lühr. Observation of lithium pick-up ions in the 5- to 20-kev energy range following the AMPTE solar wind releases. Journal of Geophysical Research: Space Physics, 91(A2):1325-1332, 1986. ISSN 2156-2202. doi: 10.1029/ JA091iA02p01325. URL http://dx.doi.org/10.1029/JA091iA02p01325.

A.F. Nagy, D. Winterhalter, K. Sauer, T.E. Cravens, S. Brecht, C. Mazelle, D. Crider, E. Kallio, A. Zakharov, E. Dubinin, M. Verigin, G. Kotova, W.I. Axford, C. Bertucci, and J.G. Trotignon. The plasma environment of mars. Space Science Reviews, 111(1-2):33-114, 2004. ISSN 0038-6308. doi: 10.1023/ B:SPAC.0000032718.47512.92. URL http://dx.doi.org/10.1023/B\%3ASPAC. 0000032718.47512 .92 .

R. Narasimha. Collisionless expansion of gases into vacuum. Journal of Spacecraft and Rockets, 12(2):294-308, 1962.

F.M. Neubauer. Nonlinear standing Alfvén wave current system at Io: Theory. Journal of Geophysical Research: Space Physics, 85(A3):1171-1178, 1980. ISSN 2156-2202. doi: 10.1029/JA085iA03p01171. URL http://dx.doi .org/10.1029/ JA085iA03p01171.

M. Neugebauer. Spacecraft observations of the interaction of active comets with the solar wind. Reviews of Geophysics, 28(2):231-252, 1990. ISSN 1944-9208. doi: 10. 1029/RG028i002p00231. URL http://dx.doi.org/10.1029/RG028i002p00231.

M. Neugebauer. The solar-wind and heliospheric magnetic field in three dimensions, pages 43-99. Springer Science Business Media, 2001. ISBN 9781852332044.

Niels F Otani. The Alfvén ion-cyclotron instability. simulation theory and techniques. Journal of Computational Physics, 78(2):251 - 277, 1988. ISSN 00219991. doi: http://dx.doi.org/10.1016/0021-9991(88)90049-6. URL http://www . sciencedirect.com/science/article/pii/0021999188900496.

M.I. Panasyuk. Galactic cosmic ray composition: From the anomalous component to the knee. In Maurice M. Shapiro, Todor Stanev, and John P. Wefel, editors, Astrophysical Sources of High Energy Particles and Radiation, volume 44 of NATO Science Series, pages 263-273. Springer Netherlands, 2001. ISBN 978-1-40200174-1. doi: 10.1007/978-94-010-0560-9_23. URL http://dx.doi.org/10.1007/ 978-94-010-0560-9_23.

K. Papadopoulos and A. T. Y. Lui. On the initial motion of artificial comets in the AMPTE releases. Geophysical Research Letters, 13(9):925-927, 1986. ISSN 1944-8007. doi: 10.1029/GL013i009p00925. URL http://dx.doi.org/10.1029/ GL013i009p00925. 
G. Paschmann, C. W. Carlson, W. Baumjohann, H. Loidl, D. W. Curtis, N. Sckopke, and G. Haerendel. Plasma observations on AMPTE/IRM during the lithium releases in the solar wind. Journal of Geophysical Research: Space Physics, 91 (A2):1271-1281, 1986. ISSN 2156-2202. doi: 10.1029/JA091iA02p01271. URL http://dx.doi.org/10.1029/JA091iA02p01271.

M. Pätzold, F. M. Neubauer, V. E. Andreev, and A. L. Gavrik. Detection of the inner plasma pileup region at Comet Halley during the Vega 1 flyby by the radio sounding experiment. , 102:2213-2222, February 1997. doi: 10.1029/96JA03140.

J. L. Phillips, J. G. Luhmann, C. T. Russell, and K. R. Moore. Finite larmor radius effect on ion pickup at Venus. Journal of Geophysical Research: Space Physics, 92(A9):9920-9930, 1987. ISSN 2156-2202. doi: 10.1029/JA092iA09p09920. URL http://dx.doi.org/10.1029/JA092iA09p09920.

Channon P. Price, Daniel W. Swift, and Lou-Chuang Lee. Numerical simulation of nonoscillatory mirror waves at the earth's magnetosheath. Journal of Geophysical Research: Space Physics, 91(A1):101-112, 1986. ISSN 2156-2202. doi: 10.1029/ JA091iA01p00101. URL http://dx.doi .org/10.1029/JA091iA01p00101.

I. Richter, C. Koenders, K. H. Glassmeier, B. T. Tsurutani, and R. Goldstein. Deep Space 1 at comet 19P/Borrelly: Magnetic field and plasma observations. , 59: 691-698, June 2011. doi: 10.1016/j.pss.2011.02.001.

I. Richter, C. Koenders, H.-U. Auster, D. Fruehauff, C. Goetz, P. Heinisch, C. Perschke, U. Motschmann, B. Stoll, K. Altwegg, J. Burch, C. Carr, E. Cupido, A. Eriksson, P. Henri, R. Goldstein, J.-P. Lebreton, P. Mokashi, Z. Nemeth, H. Nilsson, M. Rubin, K. Szegö, B. T. Tsurutani, C. Vallat, M. Volwerk, and K.-H. Glassmeier. Observation of a New Type of Low Frequency Waves at Comet 67P/Churyumov-Gerasimenko. ArXiv e-prints, May 2015.

J. L. Roeder, H. C. Koons, R. H. Holzworth, R. R. Anderson, O. H. Bauer, D. A. Gurnett, G. Haerendel, B. Häusler, and R. Treumann. Electron cyclotron harmonic waves observed by the AMPTE-IRM plasma wave experiment following a lithium release in the solar wind. Journal of Geophysical Research: Space Physics, 92(A6):5768-5776, 1987. ISSN 2156-2202. doi: 10.1029/JA092iA06p05768. URL http://dx.doi.org/10.1029/JA092iA06p05768.

M. Rubin, M. R. Combi, L. K. S. Daldorff, T. I. Gombosi, K. C. Hansen, and V. M. Tenishev. Kelvin-Helmholtz instabilities at the magnetic cavity boundary of comet 67P/Churyumov-Gerasimenko. In EPSC-DPS Joint Meeting 2011, page 260, October 2011.

C. T. Russell, H. Y. Wei, M. M. Cowee, F. Neubauer, and M. K. Dougherty. Ion Cyclotron Waves at Titan: Harbingers of Atmospheric Loss. European Planetary Science Congress 2014, EPSC Abstracts, Vol. 9, id. EPSC2014-19, 9:EPSC201419, April 2014. 
C.T. Russell and X. Blanco-Cano. Ion-cyclotron wave generation by planetary ion pickup. Journal of Atmospheric and Solar-Terrestrial Physics, 69 (14):1723 - 1738, 2007. ISSN 1364-6826. doi: http://dx.doi.org/10.1016/ j.jastp.2007.02.014. URL http://www.sciencedirect.com/science/article/ pii/S1364682607001903. Pc1 Pearl Waves: Discovery, Morphology and PhysicsPc1 Pearl Waves: Discovery, Morphology and Physics.

C.T. Russell, M.G. Kivelson, K.K. Khurana, and D.E. Huddleston. Magnetic fluctuations close to Io: ion cyclotron and mirror mode wave properties. Planetary and Space Science, 47(12):143 - 150, 1998. ISSN 0032-0633. doi: http://dx.doi.org/10. 1016/S0032-0633(98)00090-7. URL http://www.sciencedirect.com/science/ article/pii/S0032063398000907.

C.T. Russell, S.S. Mayerberger, and X. Blanco-Cano. Proton cyclotron waves at Mars and Venus. Advances in Space Research, 38(4):745 - 751, 2006. ISSN 0273-1177. doi: http://dx.doi.org/10.1016/j.asr.2005.02.091. URL http: //www.sciencedirect.com/science/article/pii/S0273117705002607. Mercury, Mars and Saturn.

A. Sadovski and A. Skalsky. Coupling of earth's magnetosphere, solar wind and lunar plasma environment. Advances in Space Research, 54(10):2017 - 2020, 2014. ISSN 0273-1177. doi: http://dx.doi.org/10.1016/j.asr.2013.07.028. URL http: //www.sciencedirect.com/science/article/pii/S0273117713004572. Lunar Science and Exploration.

R.Z. Sagdeev. Cooperative phenomena and shock waves in collisionless plasmas. Rev. Plasma Phys. (USSR)(Engl. Transl.), Vol: 4, Jan 1966.

Menelaos Sarantos, James A. Slavin, Mehdi Benna, Scott A. Boardsen, Rosemary M. Killen, David Schriver, and Pavel Trávníček. Sodium-ion pickup observed above the magnetopause during messenger's first Mercury flyby: Constraints on neutral exospheric models. Geophysical Research Letters, 36(4), 2009. ISSN 1944-8007. doi: 10.1029/2008GL036207. URL http://dx. doi .org/10.1029/2008GL036207. L04106.

Manfred Scholer. Injection and acceleration processes in corotating interaction regions: Theoretical concepts. Space Science Reviews, 89(1-2):105-114, 1999. ISSN 0038-6308. doi: 10.1023/A:1005228819169. URL http://dx.doi.org/10.1023/ A\%3A1005228819169.

G. Schwehm. Twenty years after Giotto - ESA's pioneering mission to comet Halley. ESA Bulletin, 125:8-14, February 2006.

V. D. Shapiro, V. I. Shevchenko, A. S. Sharma, K. Papadopoulos, R. Z. Sadgeev, and V. B. Lebedev. Lower hybrid turbulence at cometary bow wave and acceleration of cometary protons. Journal of Geophysical Research: Space Physics, 98(A2): 1325-1331, 1993. ISSN 2156-2202. doi: 10.1029/92JA01729. URL http://dx. doi . org/10.1029/92JA01729. 
V. D. Shapiro, R. Bingham, J. M. Dawson, Z. Dobe, B. J. Kellett, and D. A. Mendis. Energetic electrons produced by lower hybrid waves in the cometary environment and soft $\mathrm{x}$ ray emission: Bremsstrahlung and $\mathrm{k}$ shell radiation. Journal of Geophysical Research: Space Physics, 104(A2):2537-2554, 1999. ISSN 2156-2202. doi: 10.1029/1998JA900047. URL http://dx.doi.org/10.1029/1998JA900047.

V. I. Shevchenko, V. D. Shapiro, S. K. Ride, and M. Baine. Upstream wave activity at comet P/Grigg-Skjellerup. Journal of Geophysical Research: Space Physics, 100(A2):1735-1744, 1995. ISSN 2156-2202. doi: 10.1029/94JA02418. URL http: //dx.doi.org/10.1029/94JA02418.

B. Sholes, C. Zeller, and B. Matheson. Hydrazine thruster plume contamination analysis for the kepler photometer. In A. Wilson, editor, Proceedings of the 3rd European Workshop on Hydrazine. ESA, June 2004.

G.L. Siscoe and N.R. Mukherjee. Upper limits on the lunar atmosphere determined from solar wind measurements. Journal of Geophysical Research, 77(31):60426051, 1972.

Ed C. Sittler, R. E. Johnson, S. Jurac, J. D. Richardson, M. McGrath, F. Crary, D. T. Young, and J. E. Nordholt. Pickup ions at dione and enceladus: Cassini plasma spectrometer simulations. Journal of Geophysical Research: Space Physics, 109(A1):n/a-n/a, 2004. ISSN 2156-2202. doi: 10.1029/2002JA009647. URL http://dx.doi.org/10.1029/2002JA009647. A01214.

D.J. Southwood and M.G. Kivelson. Mirror instability: 1. physical mechanism of linear instability. JGR, 98(A6), 1993.

E. C. Stone, A. C. Cummings, F. B. McDonald, B. C. Heikkila, N. Lal, and W. R. Webber. Voyager 1 explores the termination shock region and the heliosheath beyond. Science, 309(5743):2017-2020, 2005. doi: 10.1126/science.1117684. URL http://www . sciencemag. org/content/309/5743/2017. abstract.

K. Szegö, K.-H. Glassmeier, R. Bingham, A. Bogdanov, C. Fischer, G. Haerendel, A. Brinca, T. Cravens, E. Dubinin, K. Sauer, L. Fisk, T. Gombosi, N. Schwadron, P. Isenberg, M. Lee, C. Mazelle, and Möbius, E. and Motschmann, U. and Shapiro, V. D. and Tsurutani, B. and Zank, G. Physics of mass loaded plasmas. Space Science Reviews, 94:429-671, 2000. ISSN 1572-9672. doi: 10.1023/A:1026568530975.

M. Tajmar. Electric propulsion plasma simulations and influence on spacecraft charging. Journal of Spacecraft and Rockets, 39(6):226-893, 2002.

L. C. Tan, G. M. Mason, and B. T. Tsurutani. Evidence for proton cyclotron waves near comet giacobini-zinner. Geophysical Research Letters, 20(3):169-172, 1993. ISSN 1944-8007. doi: 10.1029/93GL00069. URL http://dx.doi.org/10.1029/ 93GL00069.

T. Terasawa, S. Takahashi, W. Miyake, T. Mukai, M. Kitayama, and K. Hirao. Ionpickup/mass-loading process around Halley observed by Suisei. In B. Battrick, 
E. J. Rolfe, and R. Reinhard, editors, ESLAB Symposium on the Exploration of Halley's Comet, volume 250 of ESA Special Publication, pages 281-283, December 1986.

R. L. Tokar, R. J. Wilson, R. E. Johnson, M. G. Henderson, M. F. Thomsen, M. M. Cowee, E. C. Sittler, D. T. Young, F. J. Crary, H. J. McAndrews, and H. T. Smith. Cassini detection of water-group pick-up ions in the enceladus torus. Geophysical Research Letters, 35(14), 2008. ISSN 1944-8007. doi: 10.1029/2008GL034749. URL http://dx.doi.org/10.1029/2008GL034749. L14202.

Pavel M. Trávníček, David Schriver, Petr Hellinger, David Herčík, Brian J. Anderson, Menelaos Sarantos, and James A. Slavin. Mercury's magnetospheresolar wind interaction for northward and southward interplanetary magnetic field: Hybrid simulation results. Icarus, 209(1):11 - 22, 2010. ISSN 0019-1035. doi: http: //dx.doi.org/10.1016/j.icarus.2010.01.008. URL http://www.sciencedirect. com/science/article/pii/S0019103510000187. Mercury after Two MESSENGER Flybys.

W.-L. Tseng, W.-H. Ip, and A. Kopp. Exospheric heating by pickup ions at titan. Advances in Space Research, 42(1):54 - 60, 2008. ISSN 0273-1177. doi: http: //dx.doi.org/10.1016/j.asr.2008.03.009. URL http://www.sciencedirect.com/ science/article/pii/S027311770800149X.

K. Tsubouchi. Particle acceleration at corotating interaction regions in the heliosphere. The Astrophysical Journal, 795(1):47, 2014. URL http://stacks.iop. org $/ 0004-637 X / 795 / i=1 / a=47$.

Bruce T. Tsurutani. Comets: a Laboratory for Plasma Waves and Instabilities, pages 189-209. American Geophysical Union, 2013. ISBN 9781118663660. doi: 10.1029/GM061p0189. URL http://dx.doi.org/10.1029/GM061p0189.

V. Izmodenov, Y. Malama, G. Gloeckler, and J. Geiss. Filtration of interstellar $\mathrm{H}, \mathrm{O}, \mathrm{N}$ atoms through the heliospheric interface: Inferences on local interstellar abundances of the elements. AA, 414(3):L29-L32, 2004. doi: 10.1051/0004-6361: 20031697. URL http://dx.doi.org/10.1051/0004-6361:20031697.

M. I. Verigin, A. A. Galeev, R. Grar, K. I. Gringauz, E. G. Eroshenko, S. I. Klimov, M. E. Mogilevsky, E. P. Remizov, W. Riedler, R. Z. Sagdeev, S. P. Savin, K. Szego, A. Y. Sokolov, M. Tatrallyay, and K. Schwingenshuh. Physical processes in the vicinity of the cometopause: direct measurements of plasma, magnetic field, and waves by Vega-2. Soviet Astronomy Letters, 13:382, October 1987.

Z. Wang and M.G. Kivelson. Asteroid interaction with solar wind. Journal of Geophysical Research, 101(A11):24479-24493, 1996.

H. Y. Wei and C. T. Russell. Proton cyclotron waves at Mars: Exosphere structure and evidence for a fast neutral disk. Geophysical Research Letters, 33(23), 2006. ISSN 1944-8007. doi: 10.1029/2006GL026244. URL http://dx.doi.org/10. 1029/2006GL026244. L23103. 
H.Y. Wei, C.T. Russell, T.L. Zhang, and X. Blanco-Cano. Comparative study of ion cyclotron waves at Mars, Venus and Earth. Planetary and Space Science, 59(10):1039 - 1047, 2011. ISSN 0032-0633. doi: http://dx.doi.org/10.1016/j. pss.2010.01.004. URL http://www.sciencedirect.com/science/article/pii/ S0032063310000164. Comparative Planetology: Venus-Earth-Mars.

C. S. Weller and R. R. Meier. Observations of helium in the interplanetary/interstellar wind - the solar wake effect. The Astrophysical Journal, 193: 471-476, 1974.

K.-P. Wenzel. The ICE spacecraft's encounter with Comet Giacobini Zinner The first visit to a comet. ESA Bulletin, 44:32-39, November 1985.

D. Winske and S. P. Gary. Electromagnetic instabilities driven by cool heavy ion beams. Journal of Geophysical Research: Space Physics, 91(A6):6825-6832, 1986. ISSN 2156-2202. doi: 10.1029/JA091iA06p06825. URL http://dx.doi .org/10 . 1029/JA091iA06p06825.

C. S. Wu and R. C. Davidson. Electromagnetic instabilities produced by neutralparticle ionization in interplanetary space. Journal of Geophysical Research, 77 (28):5399-5406, 1972. ISSN 2156-2202. doi: 10.1029/JA077i028p05399. URL http://dx.doi.org/10.1029/JA077i028p05399.

K. Yumoto, T. Saito, and T. Nakagawa. Hydromagnetic Waves Associated with Cometary Water Group Ions - Sakigake Observations. , 187:117, November 1987.

G. P. Zank, H. L. Pauls, I. H. Cairns, and G. M. Webb. Interstellar pickup ions and quasi-perpendicular shocks: Implications for the termination shock and interplanetary shocks. Journal of Geophysical Research: Space Physics, 101 (A1):457-477, 1996. ISSN 2156-2202. doi: 10.1029/95JA02860. URL http: //dx.doi.org/10.1029/95JA02860.

G. P. Zank, A. S. Lipatov, and H. Müller. The interaction of heavy interstellar atoms with the heliosphere. AIP Conference Proceedings, 471(1):811-814, 1999. doi: http://dx.doi.org/10.1063/1.58661. URL http://scitation. aip.org/content/ aip/proceeding/aipcp/10.1063/1.58661.

Yinghui Zhang, W. J. Raitt, and D. C. Thompson. Models and numerical simulation of velocity distribution of pickup ions at low-Earth orbit. Journal of Geophysical Research: Space Physics, 106(A12):29843-29858, 2001. ISSN 2156-2202. doi: 10.1029/1999JA000280. URL http://dx.doi.org/10.1029/1999JA000280. 